Pacific Journal of Mathematic 


\title{
NONSOLVABLE FINITE GROUPS ALL OF WHOSE LOCAL SUBGROUPS ARE SOLVABLE, V
}

\author{
JoHN G. THOMPSON
}

The earlier papers in this series have reduced the problem of characterizing the minimal simple groups to several stubborn special cases. This paper handles some of these special cases. Almost all the difficulties of this paper involve groups of order $2^{a} .3^{b}$ for which $\min (a, b)$ is rather small.

This paper is a continuation of its predecessors. ${ }^{1}$ All of the results of this paper are proved on the hypothesis that $2 \in \pi_{4}$, and most of the results are proved on the additional hypothesis that $e=2$.

For $i=0,1,2$, let $\sigma_{i}$ be the set of all odd primes $p$ in $\pi(\$)$ such that $e(p)=i$. By Theorem 13.8, $\pi(\&)=\{2\} \cup \sigma_{0} \cup \sigma_{1} \cup \sigma_{2}$.

We first record some useful results about $\pi_{2}$.

LEMma 14.1. Suppose $p \in \pi_{2}$ and $\mathfrak{P}$ is a $S_{p}$-subgroup (S. If $\mathfrak{P}^{\prime} \neq 1$, then $\boldsymbol{Z}(\mathfrak{P})$ is cyclic, and in addition, for each non central subgroup $\mathfrak{A}$ of $\mathfrak{P}$ of order $p, \Omega_{1}\left(\boldsymbol{C}_{\mathfrak{P}}(\mathfrak{U})\right)=\mathfrak{A} \times \mathfrak{X}$, where $\mathfrak{X}=\Omega_{1}(\boldsymbol{Z}(\mathfrak{P}))$ and $\boldsymbol{A}_{\mathfrak{P}}(\mathfrak{U} \mathfrak{X})=\boldsymbol{A}(\mathscr{C})$, where $\mathscr{C}$ is the chain $\mathfrak{U X} \supset \mathfrak{X} \supset 1$.

Proof. Since $2 \in \pi_{4}, \quad p$ is odd. Suppose $Z(\Re)$ is non cyclic. Since $\mathfrak{P}$ has no elementary subgroup of order $p^{3}$, $\mathfrak{P}$ has exactly $1+p$ subgroups of order $p$, each of which is central. By Theorem 3.2 of [5], $\mathfrak{P}$ is metacyclic. By $0.3 .8, \mathfrak{P}^{\prime}=1$.

Suppose $\mathfrak{P}^{\prime} \neq 1$. By the preceding paragraph, $\mathfrak{X}=\Omega_{1}(Z(\mathfrak{P}))$ is of order $p$. Let $\mathfrak{A}$ be a non central subgroup of $\mathfrak{P}$ of order $p$. Let $\mathfrak{C}=C_{\mathfrak{B}}(\mathfrak{U})$. Since $\mathfrak{A} \neq \mathfrak{X}$, $\mathfrak{C}$ is a proper subgroup of $\mathfrak{P}$. Also, $\mathfrak{A} \mathfrak{X} \subseteq$ $\Omega_{1}(\mathbb{E})$. Since $\mathbb{C}$ has no elementary subgroup of order $p^{3}$, we get $\mathfrak{U X}=\Omega_{1}(\mathfrak{E})$ char $\mathfrak{E}$. Since $\mathfrak{E} \subset \mathfrak{P}$, it follows that $\boldsymbol{A}_{\mathfrak{P}}(\mathfrak{X X}) \neq 1$. Since $A_{\mathfrak{F}}(\mathfrak{U} \mathfrak{X})$ stabilizes $\mathfrak{U X} \supset \mathfrak{X} \supset 1$, the lemma follows.

Lemma 14.2. If $p \in \pi_{2}$ and $p \geqslant 5$, then every $p$-solvable subgroup of $\mathbb{B S}$ has p-length at most 1 . If $\mathfrak{B}$ is a $S_{p}$-subgroup of $\mathbb{B}$, then elements of $\mathfrak{B}$ are $\mathbb{B}$-conjugate only if they are $N(\mathfrak{P})$-conjugate.

Proof. We may assume that $\mathfrak{P}^{\prime} \neq 1$. By 0.4.4, it follows that

1) Non solvable Finite groups all of whose local subgroups are solvable, I-IV; Bull. Amer. Math. Soc., vol. 74, no 3, May, (1968), 383-437; Pacific J. Math., vol. 33, no. 2, (1970), 451-536; Pacific J. Math., vol. 39, no. 2, (1971), 483-534. 
$l_{p}(\mathfrak{S}) \leqq 1$ for every $p$-solvable subgroup $\mathfrak{S}$ of $\mathfrak{S}$. Let $\mathfrak{X}=\Omega_{1}(\boldsymbol{Z}(\mathfrak{F}))$. If $\mathfrak{X}$ is not weakly closed in $\mathfrak{P}$, then by Lemma 14.1 and the proof of Lemma 0.8.10, $S L(2, p)$ is involved in $N(\mathfrak{Y})$ for some non identity $p$-subgroup $\mathfrak{Y}$ of $(\mathfrak{S}$. As (S) is an $N$-group, this is impossible, so $\mathfrak{X}$ is weakly closed in $\mathfrak{P}$.

Let $\mathfrak{R}=N(\mathfrak{X})$. Since $l_{p}(\mathfrak{R})=1$, elements of $\mathfrak{B}$ are $\mathfrak{R}$-conjugate only if they are $N(\mathfrak{P})$-conjugate. Thus, to complete the proof, it suffices to show that elements of $\mathfrak{P}$ which are $\mathbb{S}$-conjugate are $\mathfrak{R}$ conjugate. Suppose $P, Q \in \mathfrak{P}$, and for some $G$ in $\mathbb{B}, P=Q^{G}$. Let $\mathfrak{F}=C(P) \supseteqq\left\langle\mathfrak{X}, \mathfrak{X}^{G}\right\rangle$. Since $\mathfrak{X}$ is weakly closed in $\mathfrak{P}$, there is $C$ in $\mathbb{E}$ such that $\mathfrak{X}^{G C}=\mathfrak{X}$, so $G C=N \in \mathfrak{R}$. Hence, $P^{C}=P=Q^{G C}=Q^{N}$, so that $P$ and $Q$ are $\Re$-conjugate. The proof is complete.

Lemma 14.3. Suppose $\mathfrak{X}$ is a non identity 2-subgroup of (s) and $\mathfrak{S}$ is a $S_{2}$-subgroup of $N(\mathfrak{X})$. Let $\pi(\mathfrak{S})=\left\{p_{1}, \cdots, p_{n}\right\}$, where $p_{1}>p_{2}>\cdots>p_{n}$. Then $\mathfrak{S}^{\prime}$ is nilpotent and $\mathfrak{S}$ has a Sylow series of complexion $\left(p_{1}, \cdots, p_{n}\right)$.

Proof. By Theorem 13.8, $\subseteq$ has no elementary subgroup of order $p^{3}$ for any prime $p$. Since $|\mathfrak{S}|$ is odd, the lemma follows from Lemmas 0.8.5 and 0.8.14.

LEMMA 14.4. If $p \in \sigma_{0}$ and $\mathfrak{S}$ is a 2, p-subgroup of $(\mathfrak{B )}$, then $\mathfrak{S}$ is p-closed and every involution of $\mathfrak{S}$ inverts $\boldsymbol{O}_{p}(\mathfrak{S})$.

Proof. If $\mathfrak{X}$ is a non identity 2-subgroup of $\subseteq$, then $N_{\Im}(\mathfrak{X})$ is a 2-group, since $p \in \sigma_{0}$. By Theorem 14.4.7 of [21], it follows that $\mathfrak{S}$ is $p$-closed. Taking $\mathfrak{X}$ of order 2 shows that $\boldsymbol{O}_{p}(\subseteq) \cap \boldsymbol{C}(\mathfrak{X})=1$, so $\mathfrak{X}=\langle I\rangle$, where $I$ inverts $\boldsymbol{O}_{p}(\mathfrak{S})$.

Lemma 14.5. Suppose $p \in \sigma_{1}$. Let $\mathfrak{B}$ be a subgroup of (B) of order $p$ and let $\mathfrak{S} \in \mathcal{U}(\mathfrak{B} ; 2)$. Then one of the following holds:

(a) $p \in \pi_{1}$.

(b) $\boldsymbol{C}_{\mathfrak{s}}(\mathfrak{B})$ contains no four-subgroup.

Proof. Set $\mathfrak{E}=\boldsymbol{C}(\mathfrak{B})$. Suppose $p \notin \pi_{1}$. In this case, $S_{p}$-subgroups of $\sqrt{ }$ are non cyclic. Let $\&$ be a $S_{2, p}$-subgroup of $\sqrt{5}$. It suffices to show that $\&$ contains no four-subgroup. Suppose false and $\mathfrak{B}$ is a four-subgroup of 2 . Since $p \in \sigma_{1}$ and $S_{p}$-subgroups of $\mathbb{Z}$ are non cyclic, it follows that $O_{2}(\mathbb{R})=1$. Thus, $\mathfrak{B}$ is represented faithfully on $\boldsymbol{O}_{p}(\Re)$. By Lemma 5.34, $\boldsymbol{O}_{p}(\mathfrak{Q})$ contains a subgroup $\mathfrak{X}$ of order $p$ which admits $\mathfrak{B}$ and is not centralized by $\mathfrak{F}$. Hence, $\langle\mathfrak{X}, \mathfrak{B}\rangle=\mathfrak{X}^{*}$ is elementary of order $p^{2}$. Let $\mathfrak{B}_{0}=\boldsymbol{C}_{\mathfrak{B}}(\mathfrak{X})$, so that $\left|\mathfrak{B}_{0}\right|=2$. Hence, 
$\mathfrak{B}_{0} \in U\left(\mathfrak{B}^{*} ; 2\right)$, against $p \in \sigma_{1}$. The proof is complete.

LEMmA 14.6. Suppose $p \in \sigma_{2}$ and $\mathfrak{B}$ is an elementary subgroup of (B) of order $p^{2}$ such that $\mathrm{U}(\mathfrak{B} ; 2)$ is non trivial. Then $\boldsymbol{C}(\mathfrak{B})$ contains no elementary subgroup of order $p^{3}$.

Proof. Suppose false. Let $\mathfrak{S}$ be a maximal element of $И(\mathfrak{B} ; 2)$. By hypothesis, $\mathfrak{S} \neq 1$. Let $\mathfrak{R}=\boldsymbol{N}(\mathfrak{S})$. By Lemma 13.1, $\boldsymbol{O}_{2^{\prime}}(\mathfrak{N})=1$. By maximality of $\mathfrak{S}$, we get $\mathfrak{S}=\boldsymbol{O}_{2}(\mathfrak{R})$. Hence, $\mathfrak{B}$ is represented faithfully on $\mathfrak{S}$. Since $e=2, \mathfrak{R}$ has no elementary subgroup of order $p^{3}$.

Case 1. $\mathfrak{B}$ contains a subgroup $\mathfrak{B}_{0}$ of order $p$ such that $\boldsymbol{C}_{\mathfrak{f}}\left(\mathfrak{B}_{0}\right)$ contains an elementary subgroup $\&$ of order 8.

Let $\sqrt{ }=\boldsymbol{C}\left(\mathfrak{B}_{0}\right)$. Since $\sqrt{ } \supseteqq \boldsymbol{C}(\mathfrak{B})$, $\sqrt{5}$ contains an elementary subgroup of order $p^{3}$. Let $\mathfrak{D}$ be a $S_{2, p}$-subgroup of $\mathbb{E}$ which contains $\langle\mathfrak{F}, \mathfrak{B}\rangle$. Since $\mathfrak{D}$ has an elementary subgroup of order $p^{3}$ and $p \in \sigma_{2}$, it follows that $\boldsymbol{O}_{2}(\mathfrak{D})=1$. Hence, $(5$ is represented faithfully on $\boldsymbol{O}_{p}(\mathfrak{D})$. By Lemma 5.34, $\boldsymbol{O}_{p}(\mathfrak{D})$ contains an elementary subgroup $\mathfrak{D}_{0}$ of order $p^{3}$ on which $\left(5\right.$ acts faithfully. Then $\mathfrak{D}_{0}=\mathfrak{D}_{1} \times \mathfrak{D}_{2} \times \mathfrak{D}_{3}$, where $\left|\mathfrak{D}_{i}\right|=p$, and $\mathfrak{D}_{i}$ admits $\mathbb{F}, i=1,2,3$, and $\boldsymbol{C}_{\mathbb{E}}\left(\mathfrak{D}_{0}\right)=1$. Let $\mathfrak{F}_{i}=\boldsymbol{C}_{\mathbb{E}}\left(\mathfrak{D}_{i}\right)$, so that $\left|\xi_{i}\right|=4, i=1,2,3$. Let $\mathfrak{F}_{0}=\mathfrak{F}_{1} \cap \mathfrak{F}_{2}$. Then $\mathfrak{D}^{*}=\left\langle\mathfrak{D}_{1}, \mathfrak{D}_{2}, \mathfrak{B}_{0}\right\rangle$ is elementary of order $p^{3}$ and $\mathfrak{F}_{0} \in \boldsymbol{U}\left(\mathfrak{D}^{*} ; 2\right)$, against $p \in \sigma_{2}$. Thus, this case does not occur.

Case 2. $\quad C_{\mathfrak{5}}(\mathfrak{B})$ contains a four-subgroup $\mathfrak{B}$.

Let $\sqrt{S}=\boldsymbol{C}(\mathfrak{B})$ and let $\mathfrak{D}$ be a $S_{2, p}$-subgroup of $\mathbb{C}$ which contains $\mathfrak{B}$. Since $\mathfrak{D}$ has an elementary subgroup of order $p^{3}$ and $p \in \sigma_{2}$, we have $\boldsymbol{O}_{2}(\mathfrak{D})=1$. Hence, $\mathfrak{B}$ is represented faithfully on $\boldsymbol{O}_{p}(\mathfrak{D})$. Let $\mathfrak{D}_{0}$ be a subgroup of $\boldsymbol{O}_{p}(\mathfrak{D})$ of order $p$ which admits $\mathfrak{B}$ and is not centralized by $\mathfrak{B}$. Then $\mathfrak{B}_{0}=C_{\mathfrak{B}}\left(\mathfrak{D}_{0}\right)$ is of order 2 , $\mathfrak{D}^{*}=\left\langle\mathfrak{D}_{0}, \mathfrak{B}\right\rangle$ is elementary of order $p^{3}$ and $\mathfrak{B}_{0} \in И\left(D^{*} ; 2\right)$, against $p \in \sigma_{2}$. Thus, this case does not occur.

\section{Case 3. $p \geqslant 5$.}

Suppose $\mathfrak{S}$ contains a non cyclic abelian subgroup $\Re$ which is normalized by $\mathfrak{B}$. Let $\mathfrak{B}=\Omega_{1}(\Re)$. Since Case 1 does not occur, $\left|\boldsymbol{C}_{\mathfrak{B}}\left(\mathfrak{B}_{0}\right)\right| \leqslant 4$ for all subgroups $\mathfrak{B}_{0}$ of $\mathfrak{B}$ of order $p$. Since $p \geqslant 5$, $\mathfrak{B}$ centralizes $C_{\mathfrak{B}}\left(\mathfrak{B}_{0}\right)$ for all such subgroups $\mathfrak{B}_{0}$. Since

$$
\mathfrak{B}=\left\langle\boldsymbol{C}_{\mathfrak{B}}\left(\mathfrak{B}_{0}\right) \mid 1 \subset \mathfrak{B}_{0} \subset \mathfrak{B}\right\rangle,
$$


it follows that $\mathfrak{B}$ centralizes $\mathfrak{B}$, so that Case 2 holds. This contradiction shows that no such $\Re$ exists. Hence, every non cyclic subgroup of $\mathfrak{S}$ which admits $\mathfrak{B}$ is of symplectic type.

Let $\mathbb{B}=\Omega_{1}(Z(\mathfrak{K}))$, so that $|3|=2$. Let $\mathfrak{M}=N(3) \supseteqq N(\mathfrak{Z})$. By Lemma $13.1, \boldsymbol{O}_{2^{\prime}}(\mathfrak{M})=1$. Since $\boldsymbol{O}_{2}(\mathfrak{M})=\boldsymbol{F}(\mathfrak{M})$ is non cyclic, we get that $\boldsymbol{O}_{2}(\mathfrak{M})$ is of symplectic type. Here, we are using the maximality of $\mathfrak{S}$ to conclude that $\boldsymbol{O}_{2}(\mathfrak{M}) \subseteq \mathfrak{S}$. Thus, if $\mathfrak{M}_{2}$ is a $S_{2}$-subgroup of $\mathfrak{M}$, then $3=\Omega_{1}\left(Z\left(\mathfrak{M}_{2}\right)\right)$ char $\mathfrak{M}_{2}$. By definition of $\mathfrak{M}$, it follows that $\mathfrak{M}_{2}$ is a $S_{2}$-subgroup of $\mathfrak{B S}$. Let $\mathfrak{M}^{*}$ be an element of $\mathscr{C S S}^{(\mathbb{S})}$ which contains $\mathfrak{M}$. Since $\mathfrak{M}_{2}$ is a $S_{2}$-subgroup of $\mathbb{B}$ and $2 \in \pi_{4}$, we get $\boldsymbol{O}_{2^{\prime}}\left(\mathfrak{M}^{*}\right)=1$. Since $\mathfrak{S C O}_{2}\left(\mathfrak{M}^{*}\right) \in \boldsymbol{U}(\mathfrak{B} ; 2)$, we also have $\boldsymbol{O}_{2}\left(\mathfrak{M}^{*}\right) \subseteq \mathfrak{S}$. Since $\boldsymbol{F}\left(\mathfrak{M}^{*}\right)=\boldsymbol{O}_{2}\left(\mathfrak{M}^{*}\right)$ is non cyclic, it is of symplectic type. Now Theorems 13.5, 13.6, and 13.7 yield a contradiction.

\section{Case 4. $p=3 \in \pi_{3}$.}

Since $\mathfrak{S}=O_{2}(N(\mathfrak{S}))$ and $O_{2^{\prime}}\left(N\left(\mathfrak{S}_{)}\right)\right)=1$, it follows that $N(\mathfrak{S})$ contains an element $\mathfrak{u}$ of $\mathscr{C}(2)$. Since $C(\mathfrak{B})$ contains an elementary subgroup of order $3^{3}$, we have $\mathfrak{B} \in \mathscr{A}(3)$. Hence, $\mathfrak{B} \in \mathscr{A}^{*}(\mathfrak{S})$, by Theorem 10.8. Let $\mathfrak{M}=M(\mathfrak{B}) \supseteqq N(\mathfrak{S})$. Since $\mathfrak{u} \subseteq \mathfrak{M}$, Lemma 6.1 implies that $\boldsymbol{O}_{2}(\mathfrak{M}) \neq 1$. Hence, $e(3) \geqslant 3$, against $e(3)=2$.

Case 5. $p=3 \notin \pi_{3}$.

Since $C(\mathfrak{B})$ contains an elementary subgroup of order $3^{3}$, we get $3 \in \pi_{4}$. By Theorems 8.1 and 9.1, $2 \nsim 3$. Since $\mathfrak{B}$ is represented faithfully on $\mathfrak{S}$, it follows that $\mathfrak{S}$ contains a non cyclic abelian subgroup of order 8 . By definition of $\sim$, it follows that $\mathfrak{B}$ contains an element $B$ such that $C(B)$ contains no element of $\mathscr{U}(3)$. In particular, the center of a $S_{3}$-subgroup of $B$ is cyclic, by definition of $\mathscr{C}(3)$.

Case 5a. The four-subgroup $\mathfrak{B}$ of $\mathfrak{S}$ admits $\mathfrak{B}$.

Let $\mathfrak{B}_{0}=C_{\mathfrak{B}}(\mathfrak{B})$. Since Case 2 does not occur, $\left|\mathfrak{B}_{0}\right|=3$. Let $\mathfrak{B}=\mathfrak{B}_{0} \times \mathfrak{B}_{1}$. Then $\mathfrak{B}_{\mathfrak{B}_{1}} \cong A_{4}$. Let $\mathfrak{L}_{0}$ be a $S_{2,3}$-subgroup of $C\left(\mathfrak{B}_{0}\right)$ which contains $\mathfrak{B P}$ and let $\mathbb{Q}$ be a maximal 2, 3-subgroup of $\mathbb{S}$ which contains $\mathfrak{R}_{0}$. Since $\mathbb{Q}$ contains an elementary subgroup of order $3^{3}$ and since $3 \in \sigma_{2}$, it follows that $\boldsymbol{O}_{2}(\mathfrak{Q})=1$.

Since $2 \nsim 3, \mathfrak{Q}$ contains no non cyclic abelian subgroup of order 8. Let $\mathfrak{R}_{2}$ be a $S_{2}$-subgroup of $\mathfrak{Q}$ which contains $\mathfrak{B}$, so that $\mathfrak{B}=C_{\mathfrak{R}_{2}}(\mathfrak{B})$. As is well known, the equality forces $\mathfrak{R}_{2}$ to be of maximal class. Since $\mathfrak{B}=[\mathfrak{B}, \mathfrak{B}]$, it follows that $l_{3}(\mathfrak{R}) \geqslant 2$. Hence, $\mathfrak{R}_{2}$ is dihedral of 


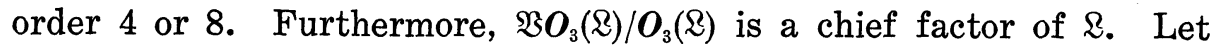
$\mathfrak{R}_{3}$ be a $S_{3}$-subgroup of $\mathbb{R}$ which contains $\mathfrak{B}$. Since $\mathfrak{R}_{2}$ is dihedral of order 4 or 8 , and since $\mathfrak{Z}$ is not 3 -closed, $N_{\&}\left(\mathfrak{R}_{3}\right)$ is a maximal subgroup of $\mathcal{L}$, and $S L(2,3)$ is not involved in $\mathcal{L}$. By Theorem 1 of

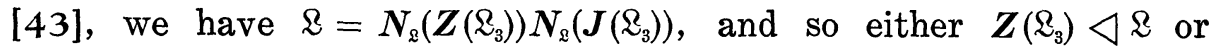
$J\left(\mathbb{R}_{3}\right) \triangleleft \mathbb{Q}$. Since $\mathfrak{Q}$ is a maximal 2, 3-subgroup of $\mathbb{S}$, it follows that $\mathfrak{R}_{3}$ is a $S_{3}$-subgroup of $\mathbb{S}$.

Case $5 \mathrm{a}(\mathrm{i}) . \quad \mathrm{O}_{3}(\mathfrak{Q})$ contains a non cyclic characteristic abelian subgroup $\mathfrak{A}$.

We may assume that $\mathfrak{A}$ is elementary. Since $\mathfrak{A}$ contains an element of $\mathscr{\mathscr { C }}\left(\mathfrak{R}_{3}\right)$, it follows that every element of $\mathfrak{U}$ centralizes an element of $\mathscr{U}\left(\mathbb{R}_{3}\right)$. Since $C(V)$ contains a non cyclic abelian subgroup of order 8 for all $V$ in $\mathfrak{B}$ and since $2 \nsim 3$, it follows that $\left|\boldsymbol{C}_{\mathfrak{r}}(V)\right| \leqq 3$ for all $V$ in $\mathfrak{B}^{\sharp}$. This implies that $\mathfrak{B B}$ acts irreducibly on $\mathfrak{A}$. Since $\mathfrak{B}_{0} \subseteq Z(\mathfrak{B P})$, it follows that $\mathfrak{B}_{0}$ centralizes $\mathfrak{N}$, so that $\left\langle\mathfrak{B}_{0}, \mathfrak{N}\right\rangle$ is elementary of order $3^{4}$. Choose $V$ in $\mathfrak{B}^{\sharp}$, and let $\mathfrak{N}_{0}=C_{\mathfrak{r}}(V)$. Since $\mathfrak{B}_{1}$ permutes $\mathfrak{B}^{\sharp}$ transitively, $\left|\mathfrak{A}_{0}\right|=3$. Hence, $\left\langle\mathfrak{A}_{0}, \mathfrak{B}_{0}\right\rangle=\mathfrak{V}_{0}$ is elementary of order $3^{2}$ and every element of $\mathfrak{E}_{0}$ centralizes an element of $\mathscr{U}(3)$. Now $C(V)$ exhibits $2 \sim 3$. This contradiction shows that this case does not arise.

Case 5 a(ii). $\quad O_{3}(\mathfrak{R})$ is of symplectic type.

Since $\mathfrak{R}_{2}$ is dihedral of order 4 or 8 , and since $\mathfrak{B}_{0}$ centralizes $\mathfrak{B}$, it follows that $\mathfrak{B}_{0} \subseteq O_{3}(\mathbb{R})$. Since $O_{3}(\mathbb{R})^{\prime}$ is of order 3 and since $\mathfrak{B}_{1} \cong A_{4}$, $\mathfrak{B}$ centralizes $\boldsymbol{O}_{3}(\mathfrak{R})^{\prime}$. Hence, $\left\langle\boldsymbol{O}_{3}(\mathfrak{R})^{\prime}, \mathfrak{B}_{0}\right\rangle\left\langle\mathfrak{R}_{3}\right.$, since $\mathfrak{R}_{3}=$ $\boldsymbol{O}_{3}(\mathbb{R}) \mathfrak{B}$. If $\mathfrak{B}_{0} \neq \boldsymbol{O}_{3}(\mathbb{R})^{\prime}$, then $\left\langle\boldsymbol{O}_{3}(\mathbb{R})^{\prime}, \mathfrak{B}_{0}\right\rangle \in \mathscr{Q}\left(\mathfrak{R}_{3}\right)$, so that $2 \sim 3$. This is not the case, so $\boldsymbol{O}_{3}(\mathbb{R})^{\prime}=\mathfrak{B}_{0}$.

Let $W=\Omega_{1}\left(\boldsymbol{O}_{3}(\mathbb{R})\right) / \mathfrak{B}_{0}, W_{0}=\boldsymbol{C}_{W}(\mathfrak{B})$. If $W_{0} \neq 1$, then $W_{0}=\mathfrak{W} / \mathfrak{B}_{0}$, where $\mathfrak{W}$ contains an element of $\mathscr{U}\left(\mathfrak{Z}_{3}\right)$. This again gives $2 \sim 3$. Hence, $W_{0}=1$. This implies that $W$ is a free $F_{3} \mathfrak{B}_{1}$-module. By Theorem 1 of $[41], C\left(\mathfrak{B}_{1}\right) \cap \Omega_{1}\left(O_{3}(\mathfrak{R})\right)$ covers $C_{W}\left(\mathfrak{B}_{1}\right)$. Thus, $\boldsymbol{O}_{3}(\mathfrak{R}) \cap C\left(\mathfrak{B}_{1}\right)$ contains an element of $\mathscr{U}\left(\Omega_{3}\right)$. This is not the case, since $C(B)$ contains no element of $\mathscr{C}(3)$ for at least one element $B$ of $\mathfrak{B}^{\#}$.

Case 5b. No four-subgroup of $\mathfrak{F}$ admits $\mathfrak{B}$.

Let $\mathfrak{M}$ be an element of $\mathscr{A S S}(\mathbb{S})$ which contains $N(\mathfrak{S})$. Since $N(\mathfrak{E})$ contains an element of $\mathscr{U}(2)$, Lemma 6.1 implies that $\boldsymbol{O}_{2}(\mathfrak{M}) \neq 1$. By Lemma 13.1, we get $\boldsymbol{O}_{2},(\mathfrak{M})=1$. Since $\mathfrak{S C O}_{2}(\mathfrak{M}) \in И(\mathfrak{B} ; 2)$, we get $\boldsymbol{O}_{2}(\mathfrak{M}) \subseteq \mathfrak{S}$, by maximality of $\mathfrak{S}$. Thus, no four-subgroup of $\boldsymbol{O}_{2}(\mathfrak{M})$ 
admits $\mathfrak{B}$. Since $\mathfrak{B}$ is elementary of order $3^{2}$, it follows that no non cyclic abelian subgroup of $\boldsymbol{O}_{2}(\mathfrak{M})$ admits $\mathfrak{B}$. Thus, $\boldsymbol{O}_{2}(\mathfrak{M})$ is of symplectic type, against Theorems $13.5,13.6$, and 13.7. The proof is complete.

Lemma 14.7. Suppose $p \in \sigma_{2}$, and $\mathfrak{B}$ is a non cyclic $p$-subgroup of (3) such that $И(\mathfrak{B} ; 2)$ is non trivial. Then Hypothesis 6.1 is satisfied with $\mathfrak{B}$ in the role of $\mathfrak{U}, \pi=\{p\}$ and $q$ any prime $\neq p$.

Proof. (a) is satisfied by our definitions; (b) and (c) are satisfied since (B) is an $N$-group. So we must verify that (d) holds.

To establish (d), we assume without loss of generality that $\mathfrak{B}$ is elementary of order $p^{2}$. This assumption is justified, since

$$
И\left(\mathfrak{B} ; p^{\prime}\right) \subseteq И\left(\mathfrak{B}_{0} ; p^{\prime}\right)
$$

for every subgroup $\mathfrak{B}_{0}$ of $\mathfrak{B}$.

Let $\mathfrak{Q} \in \boldsymbol{U}(\mathfrak{B} ; q)$ and let $\mathfrak{S}$ be a $p$-solvable subgroup of $\mathbb{S}$ which contains $\mathfrak{B}$. Suppose by way of contradiction that $\mathfrak{Q} \nsubseteq \boldsymbol{O}_{p^{\prime}}(\mathfrak{S})$. Since $\mathfrak{Q}=\left\langle C_{\mathfrak{Q}}\left(\mathfrak{B}_{0}\right) \mid 1 \subset \mathfrak{B}_{0} \subset \mathfrak{B}\right\rangle$ and since $\boldsymbol{C}_{\mathfrak{Q}}\left(\mathfrak{B}_{0}\right) \in \boldsymbol{U}(\mathfrak{B} ; q)$ for all subgroups $\mathfrak{B}_{0}$ of $\mathfrak{B}$, we can choose a subgroup $\mathfrak{B}_{0}$ of $\mathfrak{B}$ of order $p$ such that $\boldsymbol{C}_{\mathfrak{Q}}\left(\mathfrak{B}_{0}\right) \nsubseteq \boldsymbol{O}_{p^{\prime}}(\mathfrak{S})$. Set $\mathfrak{\Omega}_{0}=\boldsymbol{C}_{\mathfrak{\Omega}}\left(\mathfrak{B}_{0}\right)$, $\mathfrak{E}=\boldsymbol{C}\left(\mathfrak{B}_{0}\right)$. By Lemma 0.7.8, $\mathfrak{Q}_{0}=\boldsymbol{O}_{p},(\mathbb{S})$. Let $\mathbb{Q}$ be a $S_{p, q}$-subgroup of $\mathbb{E}$ which contains $\mathfrak{Q}_{0} \mathfrak{B}$, and let $\left\{\mathfrak{R}_{p}, \mathfrak{R}_{q}\right\}$ be a Sylow system for $\mathfrak{Z}$ with $\mathfrak{B} \subseteq \mathfrak{R}_{p}, \mathfrak{Z}_{0} \subseteq \mathfrak{R}_{q}$. By Lemma 0.7.3, $\boldsymbol{O}_{p^{\prime}}(\mathbb{R})=\mathfrak{R} \cap \boldsymbol{O}_{p^{\prime}}(\mathfrak{S})$, and so $\mathfrak{\Omega}_{0} \nsubseteq \boldsymbol{O}_{p^{\prime}}(\mathbb{R})$.

Let $\Re$ be a subgroup of $\mathfrak{Q}_{0}$ which admits $\mathfrak{B}$ and is minimal subject to $\mathfrak{R} \equiv \boldsymbol{O}_{q}(\mathfrak{R})$. Let $\Re_{0}=\mathfrak{R} \cap \boldsymbol{O}_{q}(\mathfrak{R})$. Then $\mathfrak{B}$ acts irreducibly on $\Re / \Re_{0}$ and $\Re_{0}=\boldsymbol{D}(\Re)$.

Let $\Re$ be a subgroup of $\mathbb{R}$ which contains $\Re \mathfrak{B}$ and is minimal subject to $\Re \nsubseteq \boldsymbol{O}_{q}(\Re)$. Let $\left\{\Re_{p}, \Re_{q}\right\}$ be a Sylow system of $\Re$ with $\mathfrak{B} \leqq \Re_{p}, \Re \subseteq \Re_{q}$. Let $\mathfrak{S}=\Re_{p} \cap \boldsymbol{O}_{q, p}(\Re)$. The minimality of $\Re$ forces $\Re=\mathfrak{S C R}_{q}$, and of course, $\Re_{q}=\boldsymbol{O}_{q}(\Re) \Re$. For each subset $\Re_{0}$ of $\Re$, let $\overline{\mathfrak{R}}_{0}=\boldsymbol{O}_{q}(\Re) \Re_{0} / \boldsymbol{O}_{q}(\Re)$. Thus, $\overline{\mathfrak{R}}=\overline{\mathfrak{S}} \overline{\mathfrak{R}} \overline{\mathfrak{B}}$. Also, since $\mathbb{C}=\boldsymbol{C}\left(\mathfrak{B}_{0}\right)$, we get $\mathfrak{B}_{0} \subseteq Z(\Re)$, and so $\mathfrak{B}_{0} \subseteq Z(\mathfrak{S})$.

If $\mathfrak{B} \subseteq \mathfrak{S}$, then we get $[\Re, \mathfrak{B}] \subseteq \boldsymbol{O}_{q, p}(\Re) \cap \Re \cong \Re_{0}$, so that $\mathfrak{B}$ centralizes $\Re / \Re_{0}$. Since $\Re_{0}=\boldsymbol{D}(\Re)$, it follows that $\mathfrak{B}$ centralizes $\Re$. By Lemma $5.12, \bar{\Re}$ acts faithfully on $\boldsymbol{C}_{\overline{\mathfrak{s}}}(\overline{\mathfrak{B}})$, so by $0.3 .6, \bar{\Re}$ acts faithfully on $\Omega_{1}\left(\boldsymbol{C}_{\overline{\mathfrak{F}}}(\overline{\mathfrak{B}})\right)$. Hence, $\overline{\mathfrak{B}} \subset \Omega_{1}\left(\boldsymbol{C}_{\overline{\mathfrak{F}}}(\overline{\bar{B}})\right)$. Since $\boldsymbol{C}_{\overline{\mathfrak{F}}}(\overline{\mathfrak{B}})$ and $\boldsymbol{C}_{\tilde{\mathfrak{F}}}(\mathfrak{B B})$ are incident, it follows that $\mathfrak{B} \subset \Omega_{1}\left(\boldsymbol{C}_{\mathfrak{f}}(\mathfrak{B})\right)$. This violates Lemma 14.6. Hence, $\mathfrak{B} \nsubseteq \mathfrak{F}_{\mathfrak{C}}$, so that $\mathfrak{B}_{0}=\mathfrak{B} \cap \mathfrak{S}_{\mathfrak{C}}$.

Since $\boldsymbol{C}_{\overline{\mathfrak{F}}}(\overline{\mathfrak{B}})$ and $\boldsymbol{C}_{\mathfrak{5}}(\mathfrak{B})$ are incident, it follows from Lemma 14.6 that $\mathfrak{B}_{0}=\Omega_{1}\left(C_{\mathfrak{F}}(\mathfrak{B})\right)$.

By minimality of $\Re, \bar{\Re}$ centralizes every proper subgroup of $\overline{\mathscr{S}}$ which contains $\overline{\mathfrak{B}}_{0}$ and admits $\bar{\Re} \overline{\mathfrak{B}}$. In particular, $\bar{\Re}$ centralizes 
$\left\langle\boldsymbol{D}(\overline{\mathfrak{F}}), \overline{\mathfrak{B}}_{0}\right\rangle$. Furthermore, $\overline{\mathfrak{F}}=[\overline{\mathfrak{F}}, \overline{\mathfrak{R}}] \overline{\mathfrak{B}}_{0}$, also by minimality of $\mathfrak{R}$. Since $\mathfrak{B}_{0} \subseteq \boldsymbol{Z}(\mathfrak{K})$, Lemma 0.8 .7 implies that $\boldsymbol{D}(\mathfrak{S}) \subseteq Z(\mathfrak{F})$. From 0.3.6, together with $\operatorname{cl}(\mathfrak{S}) \leqq 2$, it follows that $\mathfrak{F}$ is of exponent $p$. Hence, $\boldsymbol{C}_{\mathfrak{F}}(\mathfrak{B})=\mathfrak{B}_{0}$. Since $\mathfrak{\Re}_{p}=\mathfrak{S} \mathfrak{B}$, it follows that $\boldsymbol{C}_{\mathfrak{r}}(\mathfrak{B})=\mathfrak{B} \times \mathfrak{D}$, where $D$ is a $q$-group. Since $p$ is odd, Theorem 2 of [41] forces $\Re \cong \boldsymbol{O}_{q}(\Re)$. The proof is complete.

LEMma 14.8. Suppose $p \in \sigma_{2}$ and $\mathfrak{B}$ is an elementary subgroup of (B) of order $p^{2}$ such that $\mathrm{U}(\mathfrak{B} ; 2)$ is non trivial. Let $\mathfrak{S}$ be a maximal element of $\mathrm{U}(\mathfrak{B} ; 2)$. Then the following hold:

(a) $p \in \pi_{2}$,

(b) $N(\mathfrak{S})$ contains a $S_{p}$-subgroup of (S),

(c) If $1 \subset \mathfrak{B}_{0} \subset \mathfrak{B}$ and $C_{\mathfrak{S}}\left(\mathfrak{B}_{0}\right) \neq 1$, then $N(\mathfrak{E})$ contains a $S_{p}$-subgroup of $\boldsymbol{C}\left(\mathfrak{B}_{0}\right)$.

Proof. Set $\mathfrak{R}=N(\mathfrak{F})$. We first establish (c). Let $\mathbb{c}=\boldsymbol{C}\left(\mathfrak{B}_{0}\right)$. By Lemma $14.7, \boldsymbol{C}_{\mathfrak{g}}\left(\mathfrak{B}_{0}\right) \subseteq \boldsymbol{O}_{p^{\prime}}(\mathfrak{\mathbb { C }})$. Let $\mathbb{\Omega}_{0}$ be a $S_{2, p}$-subgroup of $O_{p^{\prime}}(\mathfrak{E}) \mathfrak{B}$ which contains $\boldsymbol{C}_{\mathfrak{f}}\left(\mathfrak{B}_{0}\right) \mathfrak{B}$. Thus, $\mathfrak{\Omega}_{0}=\mathfrak{R}_{1} \mathfrak{B}$, where $\mathfrak{R}_{1}=\mathfrak{Z}_{0} \cap \boldsymbol{O}_{p^{\prime}}(\mathbb{E})$ is a $S_{2}$-subgroup of $\boldsymbol{O}_{p^{\prime}}(\mathbb{E})$. Let $\mathbb{Q}$ be a $S_{2, p}$-subgroup of $\mathbb{E}$ which contains $\mathfrak{Z}_{0}$ and let $\left\{\mathfrak{R}_{2}, \mathfrak{R}_{p}\right\}$ be a Sylow system for $\mathfrak{R}$ with $\mathfrak{L}_{1} \subseteq \mathfrak{R}_{2}, \mathfrak{B} \subseteq \mathfrak{R}_{p}$. Then $\mathfrak{R}_{1}=\mathfrak{R} \cap \boldsymbol{O}_{p^{\prime}}(\mathfrak{S}) \triangleleft \mathfrak{R}$, and by Lemma 0.7.3, $\mathfrak{\Omega}_{1}=\boldsymbol{O}_{2}(\mathbb{R})$.

Let $\tilde{\mathcal{E}}$ be a maximal element of $U\left(\Omega_{p} ; 2\right)$ which contains $\mathfrak{L}_{1}$, and let $\tilde{\mathfrak{R}}=N(\tilde{\mathfrak{S}})$. By Lemma $14.7, \tilde{\mathfrak{F}}$ is a maximal element of $\boldsymbol{U}_{\tilde{\mathfrak{R}}}(\mathfrak{B} ; 2)$, so by Lemma $14.7, \tilde{\mathfrak{F}}$ is a maximal element of $И(\mathfrak{B} ; 2)$.

By construction, $\boldsymbol{C}_{\mathfrak{5}}\left(\mathfrak{B}_{0}\right) \neq 1, \boldsymbol{C}_{\mathfrak{\mathfrak { F }}}\left(\mathfrak{B}_{0}\right) \neq 1$. By Lemma 6.2, there is $X$ in $C(\mathfrak{B})$ such that $\mathfrak{S}_{2}=\tilde{\mathfrak{F}}^{x}$. Since $\mathbb{R}_{p}^{X}$ is a $S_{p}$-subgroup of $C\left(\mathfrak{B}_{0}\right)$, it follows that $\mathfrak{R}$ contains a $S_{p}$-subgroup of $C\left(\mathfrak{B}_{0}\right)$, namely, $\mathfrak{Q}_{p}^{X}$. So (c) holds.

Since $\mathfrak{B}$ is of type $(p, p)$, there is a subgroup $\mathfrak{B}_{0}$ of $\mathfrak{B}$ of order $p$ such that $\boldsymbol{C}_{\mathfrak{5}}\left(\mathfrak{B}_{0}\right) \neq 1$. Let $\mathfrak{N}_{p}$ be a $S_{p}$-subgroup of $\mathfrak{N}$ which contains a $S_{p}$-subgroup of $C\left(\mathfrak{B}_{0}\right)$. Let $\mathscr{B}_{p}$ be a $S_{p}$-subgroup of $\mathbb{S}^{3}$ which contains $\mathfrak{R}_{p}$. If $\mathbb{S}_{p}=\mathfrak{N}_{p}$, then both (a) and (b) hold, since $p \in \sigma_{2}$. So suppose by way of contradiction that $\mathfrak{N}_{p} \subset \mathbb{S}_{p}$.

By Lemma $14.6, \mathfrak{B} \supseteqq \Omega_{1}\left(Z\left(\mathbb{S}_{p}\right)\right)=3$, say. If $C_{\mathfrak{p}}(\mathbb{B}) \neq 1$, then we may take $\mathfrak{B}_{0}=3$ and conclude that $\mathfrak{R}$ contains an $S_{p}$-subgroup of $\boldsymbol{C}(3)$, that is, $\mathfrak{N}_{p}=\mathbb{S}_{p}$. We may therefore assume that $\boldsymbol{C}_{\mathfrak{\xi}}(3)=1$.

Let $\mathfrak{B}^{*}$ be any elementary subgroup of $\mathfrak{R}_{p}$ of order $p^{2}$. We may apply all the preceding argument with $\mathfrak{B}^{*}$ in the role of $\mathfrak{B}$, since $\mathfrak{F}$ is a maximal element of $И\left(\mathfrak{B}^{*} ; 2\right)$. We conclude that $\mathfrak{B}^{*}=\left\langle B^{*}\right\rangle \times \mathfrak{Z}$, where $\boldsymbol{C}_{\mathfrak{\xi}}\left(B^{*}\right) \neq 1$. However, we cannot assert that $\mathfrak{N}_{p}$ contains a $S_{p}$-subgroup of $C\left(B^{*}\right)$, but merely know that $\mathfrak{R}$ contains a $S_{p}$-subgroup of $C\left(B^{*}\right)$.

Let $\mathfrak{P} / \mathfrak{N}_{p}$ be a chief factor of $N_{\Theta_{p}}\left(\mathfrak{R}_{p}\right)$. Since $\mathfrak{R}_{p}$ is non cyclic 
and $p$ is odd, $\mathfrak{R}_{p}$ contains a subgroup $\mathfrak{B}^{*}$ which is elementary of order $p^{2}$ and is normal in $\mathfrak{P}$. Since $\left|\boldsymbol{C}\left(B^{*}\right)\right|_{p}=\left|\boldsymbol{C}_{\mathfrak{R}}\left(B^{*}\right)\right|_{p} \leqq|\mathfrak{N}|_{p}<|\mathfrak{P}|$, it follows that $B^{*} \notin \boldsymbol{Z}(\mathfrak{P})$.

Case 1. $\mathfrak{B}^{*} \nsubseteq Z\left(\mathfrak{N}_{p}\right)$. Since $\mathfrak{B}^{*} \triangleleft \mathfrak{P}$, we get $\left|\mathfrak{P}: C_{p}\left(\mathfrak{B}^{*}\right)\right|=p$, so that $\left|\boldsymbol{C}_{\Re}\left(B^{*}\right)\right|=\left|\mathfrak{\Re}_{p}\right|$. Since $\left|\boldsymbol{C}\left(B^{*}\right)\right|_{p}=\left|\boldsymbol{C}_{\Re}\left(B^{*}\right)\right|_{p}$, it follows that $B^{*}$

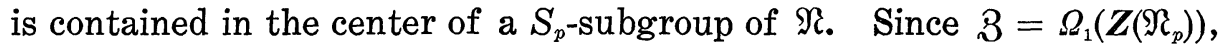
we get $\left\langle B^{*}\right\rangle_{\mathfrak{n}} Z$. Let $\mathfrak{R}_{p}^{*}$ be a $S_{p}$-subgroup of $C_{\mathfrak{n}}\left(B^{*}\right)$ which contains $\tilde{\mathfrak{N}}_{p}$, where $\tilde{\mathfrak{N}}_{p}=\boldsymbol{C}_{\mathfrak{N}_{p}}\left(B^{*}\right)$. Then $\mathfrak{B}^{*} \triangleleft\left\langle\mathfrak{N}_{p}, \mathfrak{N}_{p}^{*}\right\rangle$, $\mathfrak{N}_{p}$ maps onto the stability group of $\mathfrak{B}^{*} \supset \mathbb{Z} \supset 1$, and $\mathfrak{N}_{p}^{*}$ maps onto the stability group of $\mathfrak{B}^{*} \supset\left\langle B^{*}\right\rangle \supset 1$. Hence, $A_{\mathfrak{r}}\left(\mathfrak{B}^{*}\right)$ permutes transitively the $p+1$ subgroups of $\mathfrak{B}^{*}$ of order $p$. This is impossible, since $C_{\mathfrak{\xi}}(\mathfrak{Z})=1$, $C_{\mathfrak{5}}\left(\left\langle B^{*}\right\rangle\right) \neq 1$.

\section{Case 2. $\mathfrak{B}^{*} \subseteq Z\left(\mathfrak{\Re}_{p}\right)$.}

Since $\mathfrak{B}^{*} \subseteq Z\left(\mathfrak{R}_{p}\right)$, it follows that $\boldsymbol{A}_{\mathfrak{n}}\left(\mathfrak{B}^{*}\right)$ is a $p^{\prime}$-group. Since $\mathfrak{B}^{*}=\left\langle B^{*}\right\rangle \times \mathfrak{Z}, \quad 3 \mathfrak{K}$ is a Frobenius group and $\mathfrak{B}^{*}$ is represented faithfully on $\mathfrak{K}_{\mathfrak{C}}$, we can choose subgroups $\mathfrak{B}_{1}, \mathfrak{B}_{2}$ of order $p$ in $\mathfrak{B}^{*}$ such that $C_{\mathfrak{g}}\left(\mathfrak{B}_{i}\right) \neq 1, i=1,2, \mathfrak{B}_{i} \neq 3$, and $\mathfrak{B}_{1} \neq \mathfrak{B}_{2}$.

Since $\mathfrak{P}$ permutes transitively the subgroups of $\mathfrak{B}^{*}$ of order $p$ distinct from 3 , it follows that $\mathfrak{B}_{1}^{P}=\mathfrak{B}_{2}$ for some $P \in \mathfrak{P}-\mathfrak{N}_{p}$. Hence,

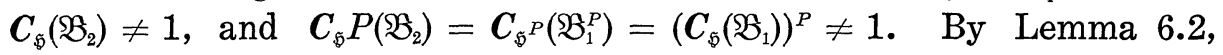
$\mathfrak{S}=\mathfrak{S}^{P C}$ for some $C$ in $C\left(\mathfrak{B}^{*}\right)$. Hence, $P C \in \mathfrak{R} \cap N\left(\mathfrak{B}^{*}\right)$, so that $A_{\mathfrak{r}}\left(\mathfrak{B}^{*}\right)$ is not a $p^{\prime}$-group. This contradiction completes the proof.

Lemma 14.9. Suppose $p \in \sigma_{2}$ and $\mathfrak{P}$ is a $S_{p}$-subgroup of (S). Let $\mathfrak{S}$ be a maximal element of $\mathrm{U}(\mathfrak{F} ; 2)$ and let $\mathfrak{R}=N(\mathfrak{S})$. Then one of the following holds:

(a) Elements of $\mathfrak{P}$ are $\mathbb{S}-$ conjugate only if they are $\mathfrak{R}$-conjugate.

(b) $\mathfrak{P}$ is abelian and if $X, Y$ are elements of $\mathfrak{P}$ which are $\mathbb{B}-$ conjugate but are not $\mathfrak{i}$-conjugate, then either $C_{\mathfrak{\$}}(X)=1$ or $C_{\mathfrak{5}}(Y)=$ 1.

Proof. Case 1. $\mathfrak{P}^{\prime}=1$.

Let $\mathbb{L}=N(\mathfrak{P})$. Since $\mathfrak{P}$ is an abelian $S_{p}$-subgroup of $\mathbb{( S )}$, elements of $\mathfrak{P}$ are $\mathbb{S}$-conjugate only if they are $\mathbb{Q}$-conjugate. Suppose $X, Y \in \mathfrak{P}$, and $X \underset{\widetilde{⿴}}{ } Y, X \underset{\Re}{\times} Y$. Choose $L$ in $\&$ with $Y=X^{L}$. By Lemma 14.8 and Lemma 14.6, $\mathfrak{S}$ and $\mathfrak{S}^{L}$ are maximal elements of $И(\mathfrak{F} ; 2)$. By Lemma 6.2, either $\boldsymbol{C}_{\mathfrak{f}}(Y)=1$ or $\boldsymbol{C}_{\mathfrak{5} L}(Y)=1$, that is, either $\boldsymbol{C}_{\mathfrak{f}}(Y)=1$ or $C_{\mathfrak{g}}(X)=1$. Thus, (b) holds in this case.

Case 2. $\mathfrak{S}^{\prime} \neq 1$. 
Let $3=\Omega_{1}(\boldsymbol{Z}(\mathfrak{P}))$. By Lemma 14.8, $p \in \pi_{2}$. By Lemma 14.1, $|3|=p$. Let $A$ be an element of $\mathfrak{B}-3$ of order $p$, and let $3=$ $\langle Z\rangle$. By Lemma 14.1, $A \widetilde{\Re}_{\mathfrak{B}} A Z^{i}$ for all $i$. This implies that $\boldsymbol{C}_{\mathfrak{f}}(A) \neq 1$, since $\langle A, Z\rangle$ is represented faithfully on $\mathfrak{S}$. Thus, by Lemma 6.2,

$$
N(\langle A, Z\rangle)=N_{\Re}(\langle A, Z\rangle) \cdot C(\langle A, Z\rangle)
$$

Case 2a. Every $p$-solvable subgroup of (s) has $p$-length at most 1.

We first show that $\mathbb{Z}$ is weakly closed in $\mathfrak{P}$. Suppose false, and $G$ in $\mathbb{B}$ satisfies $3^{G} \subseteq \mathfrak{P}, 3^{G} \neq 3$. Set $\mathfrak{X}=\mathfrak{3}^{G}, \mathfrak{B}=\mathfrak{A} \mathfrak{B}$, and let $\mathfrak{C}$ be a $S_{p}$-subgroup of $C(\mathfrak{B}) \cap N(3)$. Let $\mathfrak{B}^{*}$ be a $S_{p}$-subgroup of $\boldsymbol{C}(\mathfrak{U})$ which contains $\sqrt{ }$. Thus, $\mathbb{C} \subset \mathfrak{P}^{*}$, and $\sqrt{ }$ is not a $S_{p}$-subgroup of $N(3)$. Since $p \in \pi_{2}$, we have $\mathfrak{B}=\Omega_{1}(\mathfrak{E})$ char $\mathfrak{E}$. It follows that $A_{\mathscr{\Theta}}(\mathfrak{B})$ involves $S L(2, p)$, so $p=3$, and $l_{3}(N(\mathfrak{B}))=2$. This is not the case, so 3 is weakly closed in $\mathfrak{P}$.

Since $B$ is weakly closed in $\mathfrak{P}$, elements of $\mathfrak{P}$ are $\mathbb{B}$-conjugate only if they are $N(3)$-conjugate. Since $l_{p}(N(3))=1$, elements of $\mathfrak{P}$ are $N(\mathbb{3})$-conjugate only if they are $N(\mathfrak{P})$-conjugate. Let $\mathbb{L}=\boldsymbol{N}(\mathfrak{P})$.

First, suppose that $\mathfrak{P}$ contains a non cyclic characteristic abelian subgroup $\mathfrak{B}$. Let $\mathfrak{U}=\Omega_{1}(\mathfrak{B}), \quad \mathfrak{Q}=\boldsymbol{C}_{\mathfrak{\beta}}(\mathfrak{X})$. Then $|\mathfrak{A}|=p^{2}$ and $|\mathfrak{P}: \mathfrak{Q}|=p$. Choose $L \in \mathfrak{S}$. Then $\mathfrak{S}$ and $\mathfrak{S}^{L}$ are maximal elements

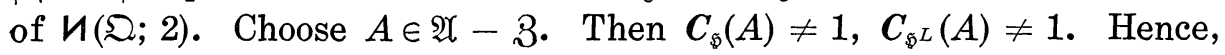
by Lemma 6.2, we get $\mathfrak{S}=\mathfrak{S}^{L C}$ for some $C$ in $C(\mathfrak{\Omega})$. Let $N=$ $L C \in \mathfrak{R}$. Thus, $L$ and $N$ induce the same automorphism of $\mathfrak{Q}$. Since $l_{p}(\Re)=1$, it follows that $\mathfrak{R} \cap \mathfrak{R}$ contains an element $N_{1}$ such that $L$ and $N_{1}$ induce the same automorphism of $\supseteq$. Let $M=N_{1} L^{-1}$. Thus, $M$ induces an automorphism of $\mathfrak{P}$ which centralizes $\mathfrak{D}$. By Lemma 0.8.12, it follows that $M$ induces an inner automorphism of $\mathfrak{P}$. Hence, $M=P D$ with $P \in \mathfrak{P}, D \in C(\mathfrak{P})$. Since $P \in \mathfrak{P} \cong \mathfrak{N}$, we get $P D=$ $N_{1} L^{-1}$, or equivalently, $D L=P^{-1} N_{1} \in \mathfrak{R}$. Thus, $\mathbb{Q}=\boldsymbol{C}(\mathfrak{P}) \cdot(\Re \cap \mathbb{R})$, so that (a) holds.

Next, suppose that $\mathfrak{P}$ is of symplectic type. Here we get $\mathfrak{P}=$ $\mathfrak{P}_{0} \mathfrak{P}_{1}$, where $\mathfrak{P}_{0}=\langle P\rangle$ is cyclic and $\mathfrak{P}_{1}$ is extra special of order $p^{3}$ and exponent $p$. If $\boldsymbol{C}_{\mathfrak{F}}\left(\mathfrak{P}^{\prime}\right) \neq 1$, then Lemma 6.2 implies that (a) holds, so we may assume that $\boldsymbol{C}_{\mathfrak{\$}}\left(\mathfrak{P}^{\prime}\right)=1$.

Let $\mathfrak{B}$ be an elementary subgroup of $\mathfrak{P}$ of order $p^{2}$ and choose $L \in$ R. Then $\mathscr{S}$ and $\mathscr{S}^{L}$ are maximal elements of $И(\mathfrak{B} ; 2)$, so by Lemma 6.2, $\mathfrak{F}=\mathfrak{S}^{L C}$ for some $C$ in $C(\mathfrak{B})$. Let $N=L C \in \mathfrak{R}$. Since $l_{p}(\mathfrak{R})=1$, we can write $N=N_{1} D$, where $N_{1} \in \mathfrak{R} \cap \mathfrak{R}$, and $D \in \boldsymbol{O}_{p^{\prime}}(\mathfrak{R})$. Hence, $L C=N_{1} D$, or equivalently, $N_{1}^{-1} L=D C^{-1}=X$, say. For each $B$ in $\mathfrak{B}, X B X^{-1}=D B D^{-1}$, since $C \in C(\mathfrak{B})$. Since $D \in \boldsymbol{O}_{p},(\mathfrak{R})$, it 
follows that $B^{-1} D B D^{-1} \in \boldsymbol{O}_{p^{\prime}}(\mathfrak{R})$. On the other hand, $B^{-1} D B D^{-1}=$ $B^{-1} X B X^{-1} \in \mathfrak{P}$, since $X=N_{1}^{-1} L \in N(\mathfrak{P})$. Hence, $X$ centralizes $\mathfrak{B}$. By 0.3.6, $X$ induces an inner automorphism of $\mathfrak{P}$, so $X=P E$ with $P \in \mathfrak{P}$, $E \in C(\mathfrak{P})$. We now get $X=N_{1}^{-1} L=P E$ or equivalently, $L E^{-1}=N_{1} P$, so that $\mathbb{Q}=(\mathbb{Q} \cap \mathfrak{R}) \boldsymbol{C}(\mathfrak{P})$, and (a) holds.

Case $2 \mathrm{~b}$. (5) has a $p$-solvable subgroup with $p$-length $\geqslant 2$.

By Lemma 14.2, we get $p=3$.

Case $2 \mathrm{~b}(\mathrm{i}) . \quad l_{3}(\mathfrak{\Re}) \geqslant 2$.

Let $\mathfrak{Q}=\mathfrak{P} \subset \boldsymbol{O}_{3^{\prime},{ }_{3}}(\mathfrak{R}), \mathfrak{R}_{0}=\boldsymbol{N}_{\mathfrak{R}}(\mathfrak{\Omega})$. Thus, $\mathfrak{Q} \subset \mathfrak{P}$ and $\mathfrak{R}=\mathfrak{R}_{0} \boldsymbol{O}_{3^{\prime}}(\mathfrak{N})$. The main difficulty here is to determine the isomorphism type of $\mathfrak{P}$.

First, suppose that $\cong$ contains a non cyclic characteristic abelian subgroup $\mathfrak{A}$. Let $\mathfrak{B}=\Omega_{1}(\mathfrak{U})$ so that $\mathfrak{B}$ char $\mathfrak{Q},|\mathfrak{B}|=3^{2}$. Let $\mathfrak{Q}^{*}=\boldsymbol{C}_{\mathfrak{B}}(\mathfrak{B})$. Since $\mathfrak{B} \triangleleft \mathfrak{N}_{0}, \mathfrak{Q}^{*}$ is a $S_{3}$-subgroup of $\boldsymbol{C}_{\mathfrak{R}_{0}}(\mathfrak{B})$. Since $\mathfrak{B}=\Omega_{1}\left(\mathfrak{\Omega}^{*}\right)$, 0.3.6 implies that $\boldsymbol{C}_{\mathfrak{R}_{0}}(\mathfrak{B})$ is $3^{\prime}$-closed. Since $\boldsymbol{O}_{3^{\prime}}\left(\boldsymbol{C}_{\mathfrak{R}_{0}}(\mathfrak{B})\right) \subseteq$ $\boldsymbol{O}_{3^{\prime}}(N)$, it follows that $\mathfrak{Q}=\mathfrak{Q}^{*}$. By Theorem 3.2 of [5], $\mathfrak{Q}$ is metacyclic. Since $l_{3}(\mathfrak{R}) \geqslant 2$, it follows that $\mathfrak{Q}$ is a homocyclic abelian group and that $|\mathfrak{P}: \mathfrak{Q}|=3$.

If $|\mathfrak{Q}|=3^{2}$, then $\mathfrak{P}$ is a non abelian group of order 27 and exponent 3. Furthermore, by (14.1), if $\mathfrak{X}$ is any subgroup of $\mathfrak{P}$ of order 9 , then $N(\mathfrak{X})=N_{\mathfrak{R}}(\mathfrak{X}) \cdot C(\mathfrak{X})$. Since $\mathfrak{Z}$ is the only subgroup of $\mathfrak{P}$ of order 9 such that $\boldsymbol{A}_{\Re}(\mathfrak{\Omega})$ involves $S L(2,3)$, it follows that $\Omega$ is weakly closed in $\mathfrak{P}$. Hence, (a) holds.

Suppose $|\mathfrak{Q}|>9$. Since $l_{3}(\mathfrak{R})=2$, it follows that $\mathfrak{O}$ is the only subgroup of $\mathfrak{P}$ of its isomorphism type, so $\mathfrak{Q}$ is weakly closed in $\mathfrak{B}$. Furthermore, if $\subseteq$ is any 3 -solvable subgroup of (s) which contains $\mathfrak{P}$, then $\mathfrak{\Omega} \subseteq \boldsymbol{O}_{3^{\prime}, 3}(\mathfrak{S})$.

Suppose $X, Y \in \mathfrak{P}$ and $X \widetilde{\mho} Y$, but $X \underset{\Re}{\nsim}$. We assume without

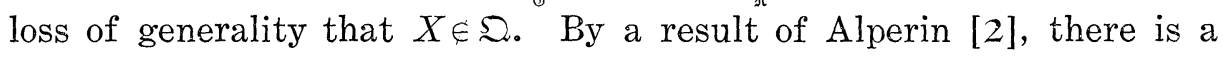
subgroup $\Re$ of $\mathfrak{R}$ with the following properties:

$(\alpha) \quad N_{\Re}(\Re)$ is a $S_{3}$-subgroup of $N(\Re)$.

( $\beta) \Re$ contains elements which are $N(\Re)$-conjugate but are not $\Re$-conjugate. Among all such $\Re$, let $|\Re|$ be maximal. Let $\Re=N(\Re)$, $\Re_{3}=\mathfrak{P} \cap \Re, \Re_{0}=\Re_{3} \cap \boldsymbol{O}_{3^{\prime}, 3}(\Re) \supseteqq \Re$. Clearly, $\Re_{0} \nsubseteq \mathfrak{Q}$, since elements of $\mathfrak{Q}$ are $\left(\mathbb{S}\right.$-conjugate only if they are $\mathfrak{R}$-conjugate. If $\boldsymbol{D}\left(\mathfrak{R}_{0}\right) \neq 1$, then $\boldsymbol{D}\left(\mathfrak{R}_{0}\right) \triangleleft\left\langle\mathfrak{R}_{0}, \mathfrak{Q}\right\rangle=\mathfrak{P}_{\text {. }} \quad$ In this case, $\boldsymbol{N}\left(\boldsymbol{D}\left(\mathfrak{R}_{0}\right)\right)$ contains $\mathfrak{P}$, so that elements of $\mathfrak{P}$ are $N\left(D\left(\Re_{0}\right)\right)$-conjugate only if they are $N(\Omega)$-conjugate. This is not the case, so $\Re_{0}$ is elementary of order 9. By (14.1), elements of $\Re_{0}$ are $\mathfrak{B}$-conjugate only if they are $\mathfrak{R}$-conjugate. Since 
elements of $\Re$ are $N(\Re)$-conjugate only if they are $N\left(\Re_{0}\right)$-conjugate, we have the desired contradiction. Thus, if $\mathfrak{Q}$ contains a non cyclic characteristic abelian subgroup, then (a) holds.

Suppose that $\mathfrak{Q}$ is of symplectic type. Then $\mathfrak{Q}=\mathfrak{Q}_{0} \mathfrak{Q}_{1}$, where $\mathfrak{\Omega}_{0}=\langle Q\rangle$ is cyclic and $\mathfrak{\Omega}_{1}$ is extra special of order 27 and exponent 3.

Let $\mathfrak{R}_{1}=\boldsymbol{O}_{3,3^{\prime}}\left(\mathfrak{N}_{0}\right)$, so that $\mathfrak{R}_{1}=\mathfrak{O M}$, where $\mathfrak{M}$ is a $S_{3}^{\prime}$-subgroup of $\mathfrak{R}_{1}$. Since $\mathfrak{M} / \boldsymbol{C}_{\mathfrak{M}}(\mathfrak{\Omega})$ is represented faithfully on $\mathfrak{\Omega}_{1}$, and since $l_{3}\left(\mathfrak{R}_{0}\right)=2$, it follows that $\mathfrak{M} / \boldsymbol{C}_{\mathfrak{R}}(\mathfrak{\Omega})$ is a quaternion group. Let $\mathfrak{A}=\boldsymbol{N}_{\mathfrak{\beta}}(\mathfrak{M})$. Thus, $\mathfrak{A} \cap \mathfrak{Z}=\mathfrak{\Omega}_{0}$, and $\mathfrak{N} / \mathfrak{\Omega}_{0}$ is of order 3 . Let $I$ be the involution of $\mathfrak{M} / C_{\mathfrak{n}}(\mathfrak{Q})$. By Lemma $5.36,\langle I\rangle$ is represented faithfully on $\boldsymbol{C}_{\mathfrak{\Omega}}(\mathfrak{X})$. Hence, $\boldsymbol{C}_{\mathfrak{0}}(\mathfrak{X})=\mathfrak{\Omega}_{0} \times \mathfrak{X}$, where $|\mathfrak{X}|=3$ and $\mathfrak{X} \times \mathfrak{Q}^{\prime}$ is the unique element of $\mathscr{\mathscr { C }}(\mathfrak{P})$. Since $3 \in \pi_{2}$, Theorem 3.2 of [5] implies that $\mathfrak{U} \cdot \boldsymbol{C}_{\mathfrak{O}}(\mathfrak{X})=\boldsymbol{C}_{\mathfrak{R}}(\mathfrak{X})$ is metacyclic. This implies that $\mathfrak{A}=\langle A\rangle$ is cyclic and that $C_{\mathfrak{P}}(\mathfrak{X})=\mathfrak{A} \times \mathfrak{X}$ is of index 3 in $\mathfrak{P}$.

Consider $N(\mathfrak{Q})$. By construction, we see that $N(\mathfrak{Q})=N_{\mathfrak{R}}(\mathfrak{\Omega})$. $(N(\mathfrak{Q}) \cap N(\mathfrak{P}))$. Since $N(\mathfrak{Q}) \cap N(\mathfrak{P}) \subseteq N\left(\mathfrak{X} \times \mathfrak{\Omega}^{\prime}\right)$ (the containment holds since $\mathfrak{X} \times \mathfrak{Q}^{\prime}$ is the unique element of $\mathscr{C}(\mathfrak{P})$ ), Lemma 6.2 implies that $N(\mathfrak{Q})=(\mathfrak{N} \cap N(\mathfrak{Q})) C(\mathfrak{Q})$. Now Lemma 6.2 implies that elements of $\mathfrak{P}$ are $\mathbb{S}$-conjugate only if they are $\mathfrak{N}$-conjugate, so (a) holds.

Case $2 \mathrm{~b}(\mathrm{ii}) . \quad l_{3}(\mathfrak{\Re})=1$.

Let $\subseteq$ be a 3 -solvable subgroup of (S) such that $l_{3}(\mathfrak{S}) \geqq 2$, and such that $|\mathfrak{S} \cap \mathfrak{P}|$ is maximal with this restriction. Let $\mathfrak{S}_{3}=\mathfrak{S} \cap \mathfrak{P}$. Since all conjugates of $\subseteq$ have 3-length at least 2, it follows that $\mathfrak{S}_{3}$ is a $S_{3}$-subgroup of $\mathfrak{S}$. Let $\tilde{\mathfrak{P}}=\mathfrak{S}_{3} \cap \boldsymbol{O}_{3^{\prime}, 3}(\mathfrak{S})$ so that $\tilde{\mathfrak{P}} \subset \mathfrak{S}_{3}$.

First, suppose $\tilde{\mathfrak{P}}$ contains a non cyclic characteristic abelian subgroup $\mathfrak{A}$. Let $\mathfrak{B}=\Omega_{1}(\mathfrak{R}), \overline{\mathfrak{P}}_{1}=C_{\tilde{\mathfrak{\beta}}}(\mathfrak{B})$. Thus, $\left|\tilde{\mathfrak{R}}: \tilde{\mathfrak{P}}_{1}\right| \geqslant 3$, and $l_{3}\left(N\left(\mathfrak{P}_{1}\right)\right) \geqslant 2$. By Lemma 6.2, $N\left(\tilde{\mathfrak{P}}_{1}\right)=\left(N\left(\tilde{\mathfrak{P}}_{1}\right) \cap \mathfrak{R}\right) \boldsymbol{C}\left(\tilde{\mathfrak{P}}_{1}\right)$. This equality forces $l_{3}(\mathfrak{N}) \geqq 2$, against $l_{3}(\mathfrak{R})=1$. Hence, $\tilde{\mathfrak{P}}$ is of symplectic type. This implies that $Z\left(\widetilde{S}_{3}\right)$ is cyclic, so that

$$
\Omega_{1}\left(Z\left(\mathfrak{S}_{3}\right)\right)=\Omega_{1}(\boldsymbol{Z}(\tilde{\mathfrak{P}}))=\Omega_{1}(Z(\mathfrak{P})) .
$$

Let $\Re=N\left(\Omega_{1}(Z(\mathfrak{P}))\right)$ so that $l_{3}(\Re) \geqq 2$. Since $\Re \cap \mathfrak{P}=\mathfrak{P}$, we assume without loss of generality that $\Omega=\subseteq$.

Choose $L \in N(\tilde{\mathfrak{P}})$, and let $\mathfrak{U}$ be the unique element of $\mathscr{U}(\mathfrak{P})$. Then $\mathfrak{S E}$ and $\mathfrak{S E}^{L}$ are maximal elements $И(\mathfrak{U} ; 2)$, so $\mathfrak{F}=\mathfrak{S}^{L C}$ for some $C$ in $\boldsymbol{C}(\mathfrak{U})$, by (14.1). Let $N=L C$. Since $l_{3}(\mathfrak{R})=1, \quad N=N_{1} D$, where $N_{1} \in \mathfrak{R} \cap N(\mathfrak{P}), D \in \boldsymbol{O}_{3^{\prime}}(\mathfrak{R})$. For each $U$ in $\mathfrak{u}, N U N^{-1}=L U L^{-1}$, $C \in \boldsymbol{C}(\mathfrak{u})$. Hence, $N U N^{-1} \in \tilde{\mathfrak{P}}$. Since $N=N_{1} D$, we get

$$
N_{1} D U D^{-1} N_{1}^{-1} \in \tilde{\mathfrak{R}} \subseteq \mathfrak{R} .
$$


Also, $N_{1} U^{-1} N_{1}^{-1} \in \mathfrak{P}$, since $N_{1} \in N_{\Re}(\mathfrak{P})$. Hence,

$$
N_{1} U^{-1} N_{1}^{-1} \cdot N_{1} D U D^{-1} N_{1}^{-1}=\left[U, D^{-1}\right]^{N_{1}} \in \mathfrak{P} \cap \boldsymbol{O}_{3^{\prime}}(\mathfrak{R})=1 .
$$

Thus, $D$ centralizes $\mathfrak{u}$. We now get $L C=N_{1} D$, or equivalently, $L=N_{1} D C^{-1}$. Since $N_{1}, D, C$ all normalize $\mathfrak{U}$, so does $\mathfrak{R}$. That is, $\boldsymbol{N}(\tilde{\mathfrak{P}}) \leqq N(\mathfrak{U})$. This is not the case, since $l_{3}(N(\tilde{\mathfrak{P}})) \geqslant 2$, so that $\mathfrak{U} \nrightarrow \boldsymbol{N}(\tilde{\mathfrak{P}})$. The proof is complete.

LEMMA 14.10. Retaining the notation of Lemma 14.9, one of the following holds:

(a) $\mathfrak{P} \subseteq \mathfrak{R}^{\prime}$,

(b) $\mathfrak{B}^{\prime}=1$.

Proof. Since $(5)$ is simple, $\mathfrak{F}=\left\langle Q^{-1} P \mid Q, P \in \mathfrak{P}, P \sim Q\right\rangle$. If $(x)$ of Lemma 14.9 holds, then $(x)$ of this lemma also holds, $x=a$ or $b$.

Hypothesis 14.1. $\sigma_{2} \neq \varnothing$.

Lemmas 14.11 through 14.26 are proved under Hypothesis 14.1.

Let $r$ be the largest prime in $\sigma_{2}$, let $\Re$ be a $S_{r}$-subgroup of (S), and let $\mathfrak{S}$ be a maximal element of $И(\Re ; 2)$. This notation is preserved through Lemma 14.26.

By Lemma 14.7, $\mathfrak{F}$ is a maximal element of $И\left(\Re_{0} ; 2\right)$ for every non cyclic subgroup $\mathfrak{R}_{0}$ of $\mathfrak{R}$. By maximality of $\mathfrak{K}$, we have $\mathfrak{K}=$ $\boldsymbol{O}_{2}(N(\mathfrak{S}))$. Since $r \in \sigma_{2}, \mathfrak{H} \neq 1$. By Lemma 13.1, $\mathfrak{R}$ is represented faithfully on $\mathfrak{S}$.

Lemma 14.11. One of the following holds:

(a) $\mathfrak{S}$ contains an element of $\mathscr{U}(2)$.

(b) $r=3$ and $\mathfrak{S}$ contain an elementary subgroup of order 8.

Proof. Suppose (a) does not hold.

Let $\mathfrak{I}$ be a $S_{2}$-subgroup of $N(\mathfrak{S})$ permutable with $\Re$, and let $\mathbb{S}_{2}$ be a $S_{2}$-subgroup of $\mathbb{S H}$ which contains $\mathfrak{T}$. Thus, $\mathfrak{S}$ contains no element of $\mathscr{U}\left(\mathbb{S}_{2}\right)$. Let $\mathfrak{L}=\mathfrak{T} \Re$.

Since $\mathfrak{F}=F(N(\mathfrak{F}))$, it follows from the maximality of $\mathfrak{S}$ that $\mathfrak{S}=\boldsymbol{F}(\mathbb{R})$. Hence, $\boldsymbol{Z}(\mathfrak{T}) \subseteq \boldsymbol{Z}(\mathfrak{S})$. Since $\mathfrak{I}$ is a $S_{2}$-subgroup of $N(\mathfrak{S})$, it follows that $\boldsymbol{Z}\left(\mathbb{S}_{2}\right) \subseteq Z(\mathfrak{Z})$. By definition of $\mathscr{U}\left(\mathbb{S}_{2}\right)$, it follows that $\boldsymbol{Z}\left(\mathbb{S}_{2}\right)$ is cyclic. Since $\boldsymbol{Z}\left(\mathscr{G}_{2}\right) \subseteq \mathfrak{F}_{2}$, it follows that $\mathfrak{I}$ contains every element of $\mathscr{\mathscr { C }}\left(\mathbb{S}_{2}\right)$.

Let $\mathfrak{U}$ be a fixed element of $\mathscr{U}\left(\mathscr{S}_{2}\right)$ and let $\mathfrak{H}_{0}=\mathfrak{u} \cap \mathfrak{S}_{\text {. }}$ Thus, $\mathfrak{U}_{0} \subset \mathfrak{u}$, and so $\mathfrak{U}_{0}=\mathfrak{U} \cap \boldsymbol{Z}\left(\mathbb{S}_{2}\right)$ is of order 2. Choose $U \in \mathfrak{U}-\mathfrak{H}_{0}$ and 
let $\mathfrak{U}_{1}=\langle U\rangle$.

By Lemma 5.36, \& contains an element $R$ of order $r$ which inverted by $U$. Let $\mathfrak{R}_{0}=\langle R\rangle, \mathfrak{S}_{0}=\left[\mathfrak{S}_{\mathcal{E}}, \mathfrak{R}_{0}\right], \mathfrak{S}_{1}=\boldsymbol{C}_{\mathfrak{g}}\left(\mathfrak{R}_{0}\right)$. Since $\left[\mathfrak{S}_{\mathcal{E}}, \mathfrak{U}_{1}\right] \subseteq \mathfrak{U}_{0}$, it follows that $\mathfrak{U}_{1}$ centralizes $\mathfrak{S} / \mathfrak{W}$, where $\mathfrak{W}=\Omega_{1}(\boldsymbol{Z}(\mathfrak{S}))$. Hence, $\mathfrak{R}_{0}$ also centralizes $\mathfrak{S} / \mathfrak{W}$, so $\mathfrak{F}_{1}$ covers $\mathfrak{S}_{\mathfrak{C}} / \mathfrak{W}$. Hence, $\mathfrak{S}_{0}=\left[\mathfrak{F}_{\mathrm{C}}, \mathfrak{R}_{0}\right]=\left[\mathfrak{W}, \mathfrak{R}_{0}\right]$, so that $\mathfrak{S}=\mathfrak{S}_{0} \times \mathscr{S}_{1}$. Since $\mathfrak{S}_{0}$ is a free $F_{2} \mathfrak{U}_{1}$-module, and since $\left[\mathfrak{S}, \mathfrak{U}_{1}\right] \leqq \mathfrak{U}_{0}$, it follows that $\left|\mathfrak{S}_{0}\right|=4$. Thus, $r=3$. Since $\mathfrak{R}$ is non cyclic and $\Re$ is represented faithfully on $\mathfrak{F}_{2}$, it follows that $\mathfrak{S}_{0} \subset \mathfrak{S}_{2}$. Since $\mathfrak{S}_{0}$ is a four-group and is a direct factor of $\mathfrak{S}$, (b) holds. The proof is complete.

Lemma 14.12. Let $q$ be an odd prime and let $\subseteq$ be a 2, q-subgroup of (S) which contains $\mathfrak{S}$. Then $\boldsymbol{O}_{2}(\mathfrak{S}) \neq 1$.

Proof. Let $\mathfrak{F}=\boldsymbol{F}(\Im)$ and suppose by way of contradiction that $\mathfrak{F}$ is a $q$-group.

By Lemma 6.1, together with $2 \in \pi_{4}$, it follows that every element $\mathfrak{u}$ of $\mathscr{U}(2)$ centralizes every element of $\mathfrak{U}\left(\mathfrak{u} ; 2^{\prime}\right)$. Since $\mathfrak{S}$ is represented faithfully on $\mathfrak{F}$, it follows that $\mathfrak{F}$ contains no element of $\mathscr{U}(2)$. By Lemma 14.11, $r=3$ and $\mathfrak{S}$ contains an elementary subgroup $\mathbb{F}$ of order 8.

Since $\mathfrak{F}$ is represented faithfully on $\mathfrak{F}$, it follows from Lemma 5.34 that $\mathfrak{F}$ contains a subgroup $\mathfrak{F}_{0}=\mathfrak{F}_{1} \times \mathfrak{F}_{2} \times \mathfrak{F}_{3}$, where $\left|\mathfrak{F}_{i}\right|=q$, $\mathfrak{F}_{i}$ admits (F, $i=1,2,3$, and $\boldsymbol{C}_{\mathbb{E}}\left(\mathfrak{\mho}_{0}\right)=1$. In particular, $q \in \pi_{3} \cup \pi_{4}$, so $q \geqslant 5$. By definition of $r$, we get $q \in \sigma_{0} \cup \sigma_{1}$. However, $\boldsymbol{C}_{\mathscr{E}}\left(\widetilde{\mho}_{1} \widetilde{\mho}_{2}\right) \neq 1$, and $\boldsymbol{C}_{\oplus}\left(\mathfrak{\Im}_{0} \mathfrak{\Im}_{1}\right) \in \boldsymbol{U}\left(\mathfrak{F}_{0} \mathfrak{\Im}_{1} ; 2\right)$, so that $q \notin \sigma_{0} \cup \sigma_{1}$. This contradiction completes the proof.

Lemma 14.13. Suppose $q$ is an odd prime $\neq r$ and $\mathfrak{B}$ is an elementary subgroup of $\mathfrak{R}$ of order $r^{2}$. If $\mathfrak{Q} \in \boldsymbol{U}(\mathfrak{B} ; q)$ and $[\mathfrak{Q}, \mathfrak{B}] \neq 1$, then $И(\Omega \mathfrak{O} ; 2)$ is trivial.

Proof. Suppose false. Let $\mathfrak{I}$ be a maximal element of $И(\mathfrak{B} \mathfrak{D} ; 2)$, so that $\mathfrak{I} \neq 1$. Hence, $q \in \sigma_{1} \cup \sigma_{2}$. Since $[\mathfrak{B}, \mathfrak{Q}] \neq 1$, Lemma 14.3 implies that $q>r$. By the maximality of $r$, we get $q \in \sigma_{1}$, so $\cong$ is cyclic.

Let $\mathfrak{B}_{0}=C_{\mathfrak{B}}(\mathfrak{Q})$. Since $\mathfrak{B}$ is elementary of order $r^{2}$, it follows that $\left|\mathfrak{B}_{0}\right|=r$. Thus, $\mathfrak{B}=\mathfrak{B}_{0} \times \mathfrak{B}_{1}$, where $\mathfrak{D}_{\mathfrak{B}_{1}}$ is a Frobenius group. Let $\mathfrak{Q}=N(\mathfrak{T})$. By Lemma 13.1, $\boldsymbol{O}_{2^{\prime}}(\mathfrak{R})=1$. Thus, $\mathfrak{B} \mathfrak{Q}$ is represented faithfully on $\mathfrak{T}=\boldsymbol{O}_{2}(\mathfrak{R})$. By Lemma $14.7, \mathfrak{T} \mathfrak{O} \subseteq \boldsymbol{O}_{r},(\mathfrak{R})$.

Let $\mathfrak{F}$ be an element of $И(\mathfrak{B} ;\{2, q\})$ which contains $\mathfrak{I} \mathfrak{O}$ and is maximal subject to $\boldsymbol{O}_{2}(\mathfrak{F}) \neq 1$. Let $\mathfrak{F}_{0}=\boldsymbol{O}_{2}(\mathfrak{F})$ and let $\Re=N\left(\mathfrak{F}_{0}\right)$. By Lemma 13.1, $\boldsymbol{O}_{2^{\prime}}(\Re)=1$. By the maximality of $\mathfrak{F}$ and Lemma 14.7, 
$\mathfrak{F}$ is a $S_{2, q^{-}}$-subgroup of $\boldsymbol{O}_{r^{\prime}}(\mathfrak{K})$. Let $\left\{\mathfrak{F}_{2}, \mathfrak{\mho}_{q}\right\}$ be a Sylow system of $\mathfrak{F}$ which admits $\mathfrak{B}$ and satisfies $\mathfrak{F} \subseteq \mathfrak{F}_{2}, \mathfrak{Q} \subseteq \mathfrak{F}_{q}$. Since $q \in \sigma_{1}$ and $\widetilde{F}_{0} \neq 1$, it follows that $S_{q}$-subgroups of $K$ are cyclic. Since $\mathfrak{B}_{1} \mathfrak{\Omega}$ is a Frobenius group, so is $\mathfrak{B}_{1} \widetilde{\mho}_{q}$ and $\widetilde{\mho}_{q}$ is a $S_{q}$-subgroup of $\Omega$.

Since $q \equiv 1(\bmod r)$, we have $q \geqslant 7$. Since $\mathfrak{F}_{q}$ is cyclic, it follows from Theorem 2 of [43] that either $\widetilde{F}=\boldsymbol{C}_{\mathfrak{⿰}}\left(\boldsymbol{Z}\left(\mathfrak{\mho}_{2}\right)\right)$ or $\mathfrak{F}=\boldsymbol{N}_{\widetilde{f}}\left(\boldsymbol{J}\left(\mathfrak{\mho}_{2}\right)\right)$. Thus, $\mathfrak{F}_{2}$ contains a characteristic subgroup $\mathfrak{C} \neq 1$ with $\mathbb{E} \triangleleft \mathfrak{F}$.

Let $\mathfrak{D}=N(\mathfrak{C})$. By the maximality of $\mathfrak{F}$ and Lemma 14.7, $\mathfrak{F}$ is a $S_{2, q^{-}}$-subgroup of $\boldsymbol{O}_{r^{\prime}}(\mathfrak{D})$. We will use this fact to show that $\widetilde{\mho}_{2}$ is

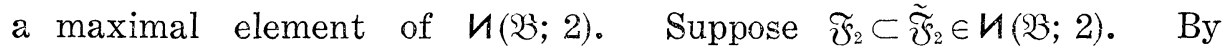
Lemma 14.7, (D) $\cap \widetilde{\mathfrak{F}}_{2} \subseteq \boldsymbol{O}_{r^{\prime}}(\mathfrak{D})$. This violates the fact that $\mathfrak{F}$ is a $S_{2, q}$-subgroup of $\boldsymbol{O}_{r}$ (D). Thus, $\mathfrak{F}_{2}$ is a maximal element of $\boldsymbol{U}(\mathfrak{B} ; 2)$.

Let $\mathfrak{S}^{*}=\left\{B \mid B \in \mathfrak{B}^{*}, C_{\widetilde{\delta}_{2}}(B) \neq 1\right\}, \quad \mathfrak{S}^{* *}=\left\{B \mid B \in \mathfrak{S}^{\sharp}, C_{\mathfrak{5}}(B) \neq 1\right\}$. Since $\mathfrak{B}_{1} \mathfrak{Q}$ is a Frobenius group which is represented faithfully on $\boldsymbol{O}_{2}(\mathfrak{F})$, it follows that $\mathfrak{B}^{*} \supseteq \mathfrak{B}-\mathfrak{B}_{0}$. Since $\mathfrak{B}$ is represented faithfully on $\mathfrak{S}$, it follows that $\mathfrak{B}^{* *} \cup\{1\}$ contains at least 2 subgroups of $\mathfrak{B}$ of order $r$. Hence, $\mathfrak{B}^{*} \cap \mathfrak{B}^{* *} \neq \varnothing$. By Lemma 6.2, the application of which is possible by Lemma 14.6, we get $\widetilde{F}_{2}=\mathfrak{S c}^{C}$ for some $C$ in $C(\mathfrak{B})$.

Since $\left(5\right.$ char $\widetilde{F}_{2}$, it follows that $\mathfrak{D} \supseteq N(\mathfrak{F})^{C}$. Since $\sqrt{5} \neq 1$, it follows that $S_{q}$-subgroups of $\mathfrak{D}$ are cyclic. Since $\mathfrak{B}_{1} \mathfrak{D}$ is a Frobenius group, $\mathfrak{B}$ does not centralize the cyclic group $\boldsymbol{O}_{q^{\prime}, q}(\mathfrak{D}) / \boldsymbol{O}_{q^{\prime}}(\mathfrak{D})$, while $\mathfrak{D}^{\prime}$ does. Hence, $\mathfrak{B} \nsubseteq \mathfrak{D}^{\prime}$. All the moreso, $\mathfrak{B} \nsubseteq N(\mathfrak{S})^{\prime}$. By Lemma $14.10, \Re^{\prime}=1$.

Since $\mathfrak{F}_{2}=\mathfrak{S C}^{C}$, it follows that $\mathfrak{B}^{*}=\mathfrak{B}^{* *}$. Choose $N \in N(\Re)$. Since $\left|\mathfrak{S}^{*}\right| \geqslant r^{2}-r$, it follows that $\mathfrak{B}^{*} \cap \mathfrak{B}^{* N} \neq \varnothing$. By Lemma 6.2, $\mathfrak{S}=\mathfrak{S}^{N C}$ for some $C$ in $C(\Re)$. Hence, $N(\Re)=(N(\Re) \cap N(\mathfrak{S})) \cdot C(\Re)$. The equality forces $\mathfrak{B} \subseteq N(\mathfrak{S})^{\prime}$, so $\mathfrak{B} \subseteq \mathfrak{D}^{\prime}$. This contradiction completes the proof.

Lemma 14.14. Let $\mathfrak{B}$ be an elementary subgroup of $\Re$ of order $r^{2}$. Let $\mathbb{C}=\boldsymbol{C}(\mathfrak{B})$. Then $\sqrt{ }$ is $r^{\prime}$-closed, $\mathfrak{S} \cap \mathbb{E}$ is a $S_{2}$-subgroup of $\mathfrak{c}$, and one of the following holds:

(a) $\boldsymbol{O}_{r^{\prime}}(\mathrm{S})$ is permutable with $\mathfrak{\mathcal { S }}$.

(b) $r=3$.

Proof. As we have already remarked several times, $\left(5\right.$ is $r^{\prime}-$ closed. Let $\mathfrak{D}=\boldsymbol{O}_{r^{\prime}}(\mathfrak{C})$ and let $\mathfrak{D}_{2}$ be a $S_{2}$-subgroup of $\mathfrak{D}$. By Lemma 6.2, $\mathfrak{D}_{2} \subseteq \mathfrak{S}^{C}$ for some $C$ in $\mathfrak{C}$. Thus, $\mathfrak{F}$ contains $\mathfrak{D}_{2}^{C-1}$, a $S_{2^{-}}$ subgroup of $\mathfrak{D}$. Hence, $\mathfrak{S} \cap \mathbb{E}$ is a $S_{2}$-subgroup of $\mathfrak{E}$, and we choose notation so that $\mathfrak{F} \cap \mathbb{C}=\mathfrak{D}_{2}$. We may assume that $r \geqslant 5$.

Let $\hat{\mathfrak{B}}=\left\{B \mid B \in \mathfrak{B}^{\sharp},\left[C_{\mathfrak{F}}(B), \mathfrak{B}\right] \neq 1\right\}$, and let $\mathfrak{S}_{0}=\left\langle C_{\mathfrak{F}}(B) \mid B \in \hat{\mathfrak{B}}\right\rangle$. 
We first show that $\mathscr{S}_{0}=\mathfrak{F}$. Namely, let $\mathfrak{S}_{1}=[\mathfrak{F}, \mathfrak{B}]$. Since $\mathfrak{B}$ is represented faithfully on $\mathfrak{S}_{\mathfrak{g}}$, it follows that $\mathfrak{S}_{1} \neq 1$. Let $V=\mathfrak{S}_{1} / \mathscr{D}\left(\mathfrak{S}_{1}\right)$, and let $V=V_{1} \times \cdots \times V_{s}$, where each $V_{i}$ is an irreducible $\mathfrak{B}$-group. Let $\mathfrak{B}_{i}=C_{\mathfrak{g}}\left(V_{i}\right)$, and let $\mathfrak{X}_{i}=C_{\mathfrak{S}}\left(B_{i}\right), 1 \leqq i \leqq s$. Since $(|\mathfrak{S}|,|\mathfrak{B}|)=1$, $\mathfrak{X}_{i}$ covers $V_{i}$. Since $\mathfrak{S}_{1}=\left[\mathfrak{S}_{1}, \mathfrak{B}\right]$, it follows that $\mathfrak{B}_{i}=\left\langle B_{i}\right\rangle$ is of order $r$ and since $\left[\mathfrak{X}_{i}, \mathfrak{B}\right] \neq 1$, we get $B_{i} \in \hat{\mathfrak{B}}$. Since $\mathfrak{S}=C_{\mathfrak{F}}(\mathfrak{B}) \cdot \mathfrak{S}_{1}$, we get that $\mathfrak{S}_{\mathfrak{S}}=\mathfrak{S}_{0}$.

We next show that if $B \in \hat{\mathfrak{B}}$, then $\mathfrak{B}$ centralizes every element

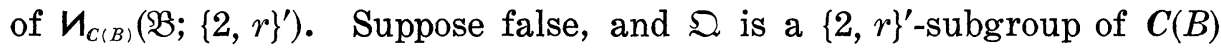
which is normalized by $\mathfrak{B}$ and is minimal subject to $[\mathfrak{B}, \mathfrak{Q}] \neq 1$. Thus, $\mathfrak{Q}$ is a $q$-group for some prime $q \neq 2, r$, and $\mathfrak{Q}=[\mathfrak{B}, \mathfrak{Q}]$. Let $\mathcal{\Omega}=\boldsymbol{C}(B)$ and let $\Re$ be a $S_{2, q}$-subgroup of $\boldsymbol{O}_{r^{\prime}}(\mathbb{\Omega})$ which is normalized by $\mathfrak{B}$. Let $\left\{\Re_{2}, \Re_{q}\right\}$ be a Sylow system for $\Re$ which admits $\mathfrak{B}$.

By Lemma 14.7, $\boldsymbol{C}_{\mathfrak{S}}(B) \subseteq \boldsymbol{O}_{r^{\prime}}(\mathfrak{\Omega}), \mathfrak{Q} \subseteq \boldsymbol{O}_{r^{\prime}}(\mathfrak{Q})$. Thus, $\mathfrak{B}$ does not centralize either $\Re_{2}$ or $\Re_{q}$. By Lemma 14.9 , we get that $\boldsymbol{O}_{2}(\Re \Re)=1$, and so $\boldsymbol{O}_{2}(\Re)=1$, as $\Re \triangleleft \Re \mathfrak{R}$.

Let $\Re_{0}=\boldsymbol{O}_{q}(\Re)$, so that $\Omega_{2}$ is represented faithfully on $\Omega_{0}$. First, suppose that $\Re_{2} \cap \boldsymbol{C}(\mathfrak{B})$ contains a four-subgroup $\mathfrak{B}$. We can then choose $V$ is $\mathfrak{B}^{\sharp}$ such that $\mathfrak{B}$ does not centralize $\Re_{0} \cap C(V)$. Let $\Omega_{0}=$ $\Re_{0} \cap C(V)$. Then $\langle V\rangle \in \boldsymbol{U}\left(\mathfrak{\Omega}_{0} \mathfrak{B} ; 2\right)$, against Lemma 14.9. Hence, $\Re_{2} \cap C(\mathfrak{B})$ contains no four-group.

Next, suppose that $\Re_{2}$ contains an abelian subgroup $\Re_{3}$ which admits $\mathfrak{B}$ and satisfies $\left[\Re_{3}, \mathfrak{B}\right] \neq 1$. Let $\Re_{3}$ be minimal with these properties. Then $\Re_{3}=\left[\Re_{3}, \mathfrak{B}\right]$ and $\mathfrak{B}_{0}=C_{\mathfrak{y}}\left(\Re_{3}\right)$ is of order $r$. Let $\mathfrak{\Omega}_{0}$ be a subgroup of $\Re_{0}$ which admits $\Re_{3} B$ and is minimal subject to $\left[\Omega_{0}, \Re_{3}\right] \neq 1$. By Lemma 0.8.7, $D\left(\Omega_{0}\right) \subseteq Z\left(\Omega_{0}\right)$, and by $0.3 .6, \Omega_{0}$ is of exponent $q$. Since $r \geqslant 5$, it follows that for some $X$ in $\Omega_{3}^{*}, \mathfrak{R}_{0} \cap C(X)$ contains an elementary subgroup of order $q^{3}$. This violates $e=2$, so this case does not occur.

Since $\mathfrak{B}$ centralizes every characteristic abelian subgroup of $\Re_{2}$, and since $C_{\Omega_{2}}(\mathfrak{B})$ contains no four-group, $\Re_{2}$ is of symplectic type. Let $\Re_{3}=\left[\Re_{2}, \mathfrak{B}\right]$, so that $\Re_{3}$ is extra special. Since $r \geqslant 5$, the width of $\Re_{3}$ is at least 2.

Let $\mathcal{R}_{1}$ be a subgroup of $\Re_{0}$ minimal subject to admitting $\Re_{3} \mathfrak{B}$ and not centralizing $\Re_{3^{\prime}}$. Then $\mathfrak{L}_{1}$ is of class at most 2 and exponent $q$, and $\Re_{3^{\prime}}=\langle I\rangle$, where $I$ inverts $\Omega_{1} / \boldsymbol{D}\left(\Omega_{1}\right)$.

Let $\mathfrak{X}$ be a four-subgroup of $\Re_{3}$ which contains $I, \mathfrak{X}=\langle X\rangle \times\langle I\rangle$. Then $X{\widetilde{\Re_{3}}} X I$ so that $C_{\mathfrak{\varepsilon}_{1}}(X)$ and $C_{\Omega_{1}}(X I)$ have the same order. Let $\mathfrak{Y}=\boldsymbol{C}_{\mathfrak{Q}_{1}}(X), \mathfrak{Y}_{1}=\boldsymbol{C}_{\mathfrak{I}_{1}}(X I)$. Since $I$ inverts $\mathfrak{\Omega}_{1} / \boldsymbol{D}\left(\mathfrak{Q}_{1}\right)$, it follows that $\left\langle\mathfrak{Y}, \mathfrak{Y} \mathfrak{Y}_{1}\right\rangle=\mathfrak{R}_{1}$. Let $\left|\mathfrak{Y}: \mathfrak{Y} \cap \boldsymbol{D}\left(\mathfrak{R}_{1}\right)\right|=q^{f}$. If $f \geqslant 3$, then $e \geqslant 3$, since $\mathfrak{R}_{1}$ is of exponent $q$. Hence, $f \leqq 2$. Since $r \geqslant 5$ and since $\mathfrak{B}$ does not centralize $\Re_{3}$, it follows that $f=2,\left|\Omega_{1}: D\left(\Omega_{1}\right)\right|=q^{4}$. Let $\tilde{\mathfrak{y}}$ be a subgroup of $\mathfrak{Y}$ which is elementary of order $q^{2}$. Then $\langle X\rangle \in \boldsymbol{U}(\tilde{\mathfrak{Y}} ; 2)$. 
Also, $\boldsymbol{C}_{\varepsilon_{1}}(\tilde{\mathfrak{y}})$ contains an elementary subgroup of order $q^{3}$. This contradicts Lemma 14.6. Thus, for each $B$ in $\hat{\mathfrak{B}}$, $\mathfrak{B}$ centralizes every element of $\boldsymbol{U}_{\boldsymbol{C}(B)}\left(\mathfrak{B}:\{2, r\}^{\prime}\right)$.

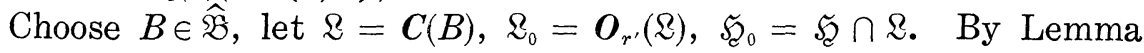
14.7, $\mathfrak{D} \subseteq \mathbb{R}_{0}, \mathfrak{S}_{0} \subseteq \mathbb{R}_{0}$. Let $\mathfrak{D} *$ be a $S_{2}$-subgroup of $\mathfrak{Z}_{0}$ which contains $\mathfrak{D}$ and admits $\mathfrak{B}$. Since $\mathfrak{B}$ centralizes every element of $\boldsymbol{K}_{\mathfrak{R}}\left(\mathfrak{B} \cdot\{2, r\}^{\prime}\right)$, it follows that $\left[\mathfrak{B}, \mathfrak{D}^{*}\right]=1$. Thus, $\mathfrak{D}^{*} \subseteq \mathfrak{E}=\boldsymbol{C}(\mathfrak{B})$. By Lemma 14.7, $\mathfrak{D}^{*} \subseteq \boldsymbol{O}_{r^{\prime}}(\mathfrak{C})$, so by definition of $\mathfrak{D}$, we get $\mathfrak{D}=\mathfrak{D}^{*}$.

Let $\mathfrak{F}_{1}$ be a $S_{2}$-subgroup of $\mathfrak{L}_{0}$ which contains $\mathfrak{S}_{0}$. We argue that $\mathfrak{S}_{0}=\mathfrak{S}_{1}$. In any case, by Lemma 6.2 , there is $C$ in $C(\mathfrak{B})$ such that $\mathfrak{S}_{1}^{C} \subseteq \mathfrak{S}_{2}$. Thus, $\mathfrak{S}_{1}^{C} \subseteq \mathfrak{S}_{0}$, by definition of $\mathfrak{S}_{0}$. Hence, $\left|\mathfrak{S}_{1}\right| \leqq\left|\mathfrak{S}_{0}\right|$, so $\mathfrak{S}_{0}=\mathfrak{S}_{1}$.

We have shown that for each $B$ in $\widehat{\mathfrak{B}}, \boldsymbol{C}_{\mathfrak{\xi}}(B)$ is permutable with D. Since $\mathfrak{S}=\left\langle C_{\mathfrak{E}}(B) \mid B \in \hat{\mathfrak{B}}\right\rangle$, the proof is complete.

LEMma 14.15. Let $\mathfrak{B}$ be an elementary subgroup of $\mathfrak{R}$ of order $r^{2}$. Let $\mathfrak{E}=\boldsymbol{C}(\mathfrak{B})$. Then $\boldsymbol{O}_{r^{\prime}}(\mathfrak{E})$ is permutable with $\mathfrak{S}$.

Proof. Suppose false. By Lemma 14.14, we have $r=3$, and so $\sigma_{2}=\{3\}$.

Case 1. $C_{\mathfrak{s}}(\mathfrak{B})$ contains a four-group. Choose $B \in \mathfrak{B}^{\ddagger}$ and let $\mathbb{Z}=\boldsymbol{C}(B), \mathbb{\Omega}_{0}=\boldsymbol{O}_{r^{\prime}}(\mathbb{\Omega})$. Choose $q \in \pi\left(\Omega_{0}\right), q \neq 2$, and let $\Omega$ be a $S_{2, q^{-}}$ subgroup of $\Omega_{0}$ which admits $\mathfrak{B}$. Let $\left\{\Re_{2}, \Re_{q}\right\}$ be a Sylow system of $\Re$ which admits $\mathfrak{B}$. By Lemma $14.7, C_{\mathfrak{F}}(\mathfrak{B}) \subseteq \mathfrak{R}_{0}$, so $\Omega_{2}$ contains a four-group $\mathfrak{B}$ which is centralized by $\mathfrak{B}$. Suppose by way of contradiction that $\left[\Re_{q}, \mathfrak{B}\right] \neq 1$. We can then choose $V$ in $\mathfrak{S}^{\sharp}$ such that $C_{\Re_{q}}(V)=\tilde{\Re}_{q}$ is not centralized by $\mathfrak{B}$. Hence, $\langle V\rangle \in \boldsymbol{U}\left(\tilde{\Omega}_{q} \mathfrak{B} ; 2\right)$, against Lemma 14.13. Thus, $\mathfrak{B}$ centralizes $\Omega_{q}$.

Let $\mathfrak{D}^{*}$ be a $S_{2^{\prime}}$-snbgroup of $\mathfrak{S}_{0}$ which contains $\mathfrak{D}$ and admits $\mathfrak{B}$; (D* exists, since by Lemma 14.7, $\mathfrak{D} \subseteq \mathbb{R}_{0}$. Here, (D) denotes a $S_{2^{\prime}}$ subgroup of $\boldsymbol{O}_{3^{\prime}}(\mathfrak{C})$. By the preceding paragraph, $\mathfrak{B}$ centralizes $\mathfrak{D}^{*}$, so by Lemma $14.7, \mathfrak{D}^{*} \subseteq \boldsymbol{O}_{3^{\prime}}(\mathfrak{E})$. Hence, $\mathfrak{D}=\mathfrak{D}^{*}$.

Since $C_{\tilde{\hat{f}}}(B)$ is a $S_{2}$-subgroup of $\Omega_{0}$, it follows that $\mathfrak{D}$ is permutable with $C_{\mathfrak{f}}(B)$. Since $\mathfrak{S E}=\left\langle C_{\mathfrak{F}}(B) \mid B \in \mathfrak{B}^{\sharp}\right\rangle$, the lemma follows.

For the remainder of the proof, we assume that $\boldsymbol{C}_{\mathfrak{5}}(\mathfrak{B})$ contains no four-group.

Case 2. $\boldsymbol{C}_{\mathfrak{5}}(\mathfrak{B})$ is not cyclic.

Let $\mathfrak{S}_{0}=\boldsymbol{C}_{\mathfrak{S}}(\mathfrak{B})$. Since $\mathfrak{S}_{0}$ contains no four-group, it follows that $\mathfrak{S}_{0}$ is a generalized quaternion group. Let

$$
\widehat{\mathfrak{B}}=\left\{B \mid B \in \mathfrak{B}^{\sharp},\left[C_{\mathfrak{F}}(B), \mathfrak{B}\right] \neq 1\right\} .
$$


As in the proof of Lemma 14.14, we get $\mathfrak{S}=\left\langle C_{\mathfrak{F}}(B) \mid B \in \widehat{\mathfrak{B}}\right\rangle$.

Choose $B$ in $\hat{\mathfrak{B}}$. We will show that $\boldsymbol{C}_{\mathfrak{f}}(B)$ contains an elementary subgroup of order 8 . Let $\mathfrak{Q}=C_{\mathfrak{F}}(B)$ and let $\mathfrak{B}_{0}$ be a subgroup of $\mathfrak{B}$ of order 3 distinct from $\langle B\rangle$. Thus, $\mathfrak{B}_{0}$ does not centralize $\mathfrak{D}$, and of course, $\mathfrak{\Omega} \supset \mathfrak{F}_{0}$. Let $\mathfrak{\Omega}_{0}=N_{\mathfrak{Q}}\left(\mathfrak{S}_{0}\right)$, and let $\mathfrak{S}_{1} / \mathfrak{F}_{0}$ be a chief factor of $\mathfrak{O}_{0} \mathfrak{B}_{0}$. Thus, $\mathfrak{S}_{1} / \mathfrak{S}_{0}$ is a four-group and $\mathfrak{S}_{0}=C_{\mathfrak{S}_{1}}\left(\mathfrak{B}_{0}\right)=C_{\mathfrak{Q}}\left(\mathfrak{B}_{0}\right)=C_{\mathfrak{g}}(\mathfrak{B})$. If $\mathfrak{S}_{0}$ is a direct factor of $\mathfrak{S}_{1}$, we are done, so suppose not. If $\mathbb{F}$ is a characteristic abelian subgroup of $\mathfrak{S}_{1}$ and $\mathbb{F} \nsubseteq \mathfrak{S}_{0}$, then $\mathfrak{B}_{0}$ does not centralize $\mathbb{F}$, so $\mathfrak{B}_{0}$ does not centralize $\Omega_{1}(\mathfrak{F})$, a four-group. This forces $\mathfrak{S}_{1}=\mathfrak{S}_{0} \times \Omega_{1}(\mathbb{F})$. Hence, every characteristic abelian subgroup of $\mathscr{S}_{1}$ is contained in $\mathscr{S}_{0}$, so is cyclic; $\mathscr{S}_{1}$ is of symplectic type. Thus, $\mathfrak{S}_{1}$ is the central product of $\mathfrak{S}_{0}$ and $\left[\mathfrak{S}_{1}, \mathfrak{B}_{0}\right]$, and $\left[\mathfrak{S}_{1}, \mathfrak{B}_{0}\right]$ is a quaternion group. As is well known, $\mathfrak{S}_{1}$ contains an elementary subgroup of order 8.

Again, let $B \in \hat{\mathfrak{B}}$, let $\mathfrak{L}=\boldsymbol{C}(B), \quad \mathfrak{Q}_{0}=\boldsymbol{O}_{3^{\prime}}(\mathfrak{\&})$. By Lemma 14.7, $\mathfrak{D}=C_{\mathfrak{s}}(B) \subseteq \Omega_{0}$, and also $\mathfrak{D} \subseteq \Omega_{0}$. Choose $q \in \pi\left(\mathbb{R}_{0}\right), q \neq 2$, and let $\Re$ be a $S_{2, q}$-subgroup of $\mathfrak{\Omega}_{0}$ which admits $\mathfrak{B}$ and contains $\mathfrak{\Omega}$. Let $\left\{\Omega_{2}, \Omega_{q}\right\}$ be a Sylow system of $\Re$ which admits $\mathfrak{B}$. Suppose $\left[\Re_{q}, \mathfrak{B}\right] \neq 1$. By Lemma 14.13 , we get $\boldsymbol{O}_{2}(\Re)=1$. Let $\&$ be an elementary subgroup of $\Omega$ of order 8. By Lemma 5.34, $O_{q}(\Re)$ contains a subgroup $\mathfrak{F}=$ $\mathfrak{F}_{1} \times \mathfrak{F}_{2} \times \mathfrak{\mho}_{3}$ such that $\left|\mathfrak{F}_{i}\right|=q$, $\mathfrak{F}_{i}$ admits $\mathbb{F}$ and $C_{\mathbb{E}}(\mathfrak{F})=1$. Thus, $\boldsymbol{C}_{\mathbb{E}}\left(\mathfrak{\mho}_{1} \widetilde{\mho}_{2}\right)=\widetilde{\mathbb{E}} \neq 1$, and $\widetilde{E} \in U\left(\mathfrak{\mho}_{1} \widetilde{\mho}_{2} ; 2\right)$. This violates $\sigma_{2}=\{3\}$. Hence, $\mathfrak{B}$ centralizes $\mathfrak{\Omega}_{q}$. Thus, $\mathfrak{B}$ centralizes a $S_{2}$-subgroup of $\mathfrak{R}_{0}$, so $\mathfrak{D}$ is a $S_{2}$-subgroup of $\mathfrak{R}_{0}$. Since $C_{\mathfrak{\$}}(B)$ is a $S_{2}$-subgroup of $\mathfrak{\Omega}_{0}$, the lemma follows.

Case 3. $C_{\mathfrak{S}}(\mathfrak{B})$ is cyclic and $И(\mathfrak{D} \mathfrak{B} ; 2)$ is non trivial.

Let $\mathfrak{X}$ be a maximal element of $И(\mathfrak{D} \mathfrak{B} ; 2)$. Since $\pi(\mathfrak{D}) \subseteq \sigma_{1} \cup \sigma_{2}$, and since $\mathfrak{D}$ is a $3^{\prime}$-group, while $\sigma_{2}=\{3\}$, it follows that $\pi(\mathfrak{D}) \leqq \sigma_{1}$; so $\mathfrak{D}$ is a $Z$-group. Let $\mathfrak{Y}=N(\mathfrak{X})$. By maximality of $\mathfrak{X}$, we have $\mathfrak{X}=\boldsymbol{O}_{2}(\mathfrak{Y )})$. By Lemma 13.1, it follows that $\boldsymbol{O}_{2^{\prime}}(\mathfrak{Y})=1$. Let $\mathfrak{Y}_{0}=\boldsymbol{O}_{3^{\prime}}(\mathfrak{Y})$. Thus, $\mathfrak{D} \subseteq \mathfrak{Y}_{0}$, by Lemma 14.11, it follows that $\mathfrak{D}$ is a $S_{2^{\prime}}$-subgroup of $\mathfrak{Y}_{0}$. By Lemma $14.7, \mathfrak{Y}_{0} \cap \mathfrak{S}=\tilde{\mathfrak{F}}$ is a $S_{2}$-subgroup of $\mathfrak{Y}_{0}$. Since $\tilde{\mathfrak{S}} / \mathfrak{X}$ is represented faithfully on $\boldsymbol{O}_{2,2^{\prime}}\left(\mathfrak{Y}_{0}\right) / \mathfrak{X}$, and since $\boldsymbol{O}_{2,2}\left(\mathfrak{Y}_{0}\right) / \mathfrak{Y}$ is a $Z$-group, it follows that $\mathfrak{B}$ centralizes $\tilde{\mathfrak{F}} / \mathfrak{X}$.

Since $C_{\mathfrak{S}}(\mathfrak{B})$ is cyclic, so is $\tilde{\mathscr{F}} / \mathfrak{X}$. Thus, $\pi(\mathfrak{D})$ contains a prime $q$ such that a $S_{q}$-subgroup $\mathfrak{D}_{q}$ of $\mathfrak{D}$ is permutable with $\tilde{\mathfrak{H}}$ and such that $\mathfrak{Y}=\boldsymbol{O}_{2}\left(\tilde{\mathfrak{S}} \mathfrak{D}_{q}\right)$. Since $q \geqslant 5$, either $\boldsymbol{Z}(\tilde{\mathfrak{F}}) \triangleleft \tilde{\mathfrak{F}} \mathfrak{D}_{q}$ or $\boldsymbol{J}(\tilde{\mathfrak{S}}) \triangleleft \tilde{\mathscr{S}} \mathfrak{D}_{q}$, by Theorem 1 of [43]. So in any case, $\tilde{\mathfrak{F}}$ contains a non identity characteristic subgroup $\mathfrak{X}_{0}$ such that $\mathfrak{X}_{0} \triangleleft \tilde{\mathscr{S}} \mathfrak{D}_{q} \mathfrak{B}$. Let $\mathfrak{M}=N\left(\mathfrak{X}_{0}\right)$. Suppose by way of contradiction that $\tilde{\mathfrak{S}} \subset \mathfrak{F}$. Then $\tilde{\mathfrak{F}} \subset \mathfrak{S} \cap \mathfrak{M}$, since 
$\mathfrak{X}_{0}$ char $\tilde{\mathfrak{F}}$. Let $\mathfrak{M}_{0}=\boldsymbol{O}_{3^{\prime}}(\mathfrak{M})$ so that by Lemma $14.7, \mathfrak{S} \cap \mathfrak{M} \cong M_{0}$,

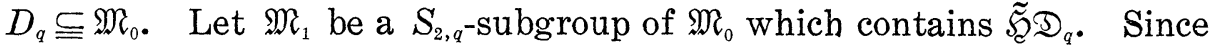
$\mathfrak{X}$ is a maximal element of $\boldsymbol{U}_{\mathfrak{n}}\left(\mathfrak{D}_{q} \mathfrak{B} ; 2\right)$, it follows that $\mathfrak{X}$ is a maximal element of $\boldsymbol{U}\left(\mathfrak{D}_{q} \mathfrak{B} ; 2\right)$. Hence, $\boldsymbol{O}_{2}\left(\mathfrak{M}_{1}\right) \subseteq \mathfrak{X}$. The reverse containment holds since $S_{q}$-subgroups of $\mathfrak{M}_{1}$ are cyclic, the cyclicity holding since $q \in \sigma_{1}$. Hence, $\boldsymbol{O}_{2}\left(\mathfrak{M}_{1}\right)=\mathfrak{X}$. But by construction, $\mathfrak{S} \cap \mathfrak{M} \supset \tilde{\mathfrak{F}}$, so $\tilde{\mathfrak{F}}$ is not a $S_{2}$-subgroup of $\mathfrak{M}_{1}$. Hence,

$$
\left|\boldsymbol{O}_{3^{\prime}}\left(N\left(\mathfrak{X}_{0}\right)\right)\right|_{2} \geqslant|\mathfrak{S} \cap \mathfrak{M}|>|\tilde{\mathfrak{G}}|=\left|\boldsymbol{O}_{3^{\prime}}\left(N\left(\mathfrak{X}_{0}\right)\right)\right|_{2} .
$$

This contradiction shows that $\tilde{S}=\tilde{\mathscr{F}}$, so that the lemma holds.

Case 4. $C_{\mathfrak{1}}(\mathfrak{B})$ is cyclic and $И(\mathfrak{D} \mathfrak{B} ; 2)$ is trivial.

First, suppose that for some $B$ in $\mathfrak{B}^{\sharp}, C_{\mathfrak{f}}(B)$ contains an elementary subgroup $\mathbb{S}$ of order 8 . Let $\mathfrak{L}=\boldsymbol{C}(B), \mathbb{\Omega}_{0}=\boldsymbol{O}_{3^{\prime}}(\mathbb{R})$. By Lemma 14.7, $\left\langle\left(\mathcal{D}, C_{\mathfrak{F}}(B)\right\rangle \subseteq \Omega_{0}\right.$. Let $q$ be an odd prime in $\pi\left(\Omega_{0}\right)$ and let $\Re$ be a $S_{2, q}$-subgroup of $\mathfrak{\Omega}_{0}$ which contains $C_{\mathfrak{F}}(\mathfrak{B})$ and admits $\mathfrak{B}$. Let $\left\{\Re_{2}, \Re_{q}\right\}$ be a Sylow system for $\Re$ which admits $\mathfrak{B}$. Suppose $\left[\mathfrak{B}, \Re_{q}\right] \neq 1$. By Lemma 14.11, we get $\boldsymbol{O}_{2}(\Re)=1$. Since $\mathfrak{F} \subseteq \Omega_{2}$, Lemma 5.34 implies that $q \in \sigma_{2}$, against $\sigma_{2}=\{3\}$. Hence, $\left[\mathfrak{B}, \Re_{q}\right]=1$, so that $\mathfrak{D}$ is a $S_{2^{\prime}}$ subgroup of $\mathbb{R}_{0}$. Since $\left[\mathfrak{F} \subseteq \mathbb{R}_{0}\right.$, it follows that $\pi(\mathbb{D}) \subseteq \sigma_{1} \cup \sigma_{2}$. Since (D) is a $3^{\prime}$-group, and $\sigma_{2}=\{3\}$, we get $\pi(\mathfrak{D}) \subseteq \sigma_{1}$. Since $(5$ is elementary of order 8 , it follows that $(D$ is a $Z$-group, Since $U(\mathfrak{B D} ; 2)$ is trivial, $\boldsymbol{F}\left(\mathbb{\Omega}_{0}\right)$ is of odd order, so is cyclic. Hence, $\left(\mathbb{\Omega}_{0} \mathfrak{B}\right)^{\prime} \leqq \boldsymbol{F}\left(\mathbb{\Omega}_{0}\right)$. In In particular, $\left[\boldsymbol{C}_{\mathfrak{S}}(B), \mathfrak{B}\right] \subseteq \boldsymbol{F}\left(\mathbb{Z}_{0}\right) \cap \mathfrak{S}=1$. This is not the case, since $C_{\mathfrak{5}}(\mathfrak{B})$ is cyclic. Thus, for each $B$ in $\mathfrak{B}^{\ddagger}, C_{\mathfrak{p}}(B)$ contains no elementary subgroup of order 8 .

Suppose $C_{\mathfrak{5}}(\mathfrak{B}) \neq 1$. In this case, it follows that every abelian subgroup of $\mathfrak{S}$ which admits $\mathfrak{B}$ is cyclic. Let $\mathfrak{M}$ be an element of $\mathscr{L S}(\mathbb{S})$ which contains $N(\mathfrak{F})$. Since $\mathfrak{M}$ contains an element of $\mathscr{U}(2)$, it follows that $\boldsymbol{O}_{2}(\mathfrak{M}) \neq 1$. By Lemma 13.1, $\boldsymbol{O}_{2^{\prime}}(\mathfrak{M})=1$. Hence, $\boldsymbol{O}_{2}(\mathfrak{M})$ is of symplectic type, against Theorems 13.5, 13.6, 13.7. Hence, $\boldsymbol{C}_{\mathfrak{5}}(\mathfrak{B})=1$.

Suppose $B \in \mathfrak{B}^{\ddagger}$ and $C_{\mathfrak{F}}(B)$ is non abelian. Let $\mathfrak{S}_{0}=C_{\mathfrak{s}}(B)$, and choose $B_{1} \in \mathfrak{B}-\langle B\rangle$. Then $\mathscr{F}_{0}\left\langle B_{1}\right\rangle$ is a Frobenius group, so $Z\left(\mathscr{S}_{0}\right)$ is non cyclic. Let $\mathbb{R}=\boldsymbol{C}(B), \mathfrak{\Omega}_{0}=\boldsymbol{O}_{3^{\prime}}(\mathfrak{R})$. By Lemma 14.7, $\left\langle\mathcal{D}, \mathfrak{S}_{0}\right\rangle \subseteq \mathfrak{\Omega}_{0}$. Let $\mathfrak{F}=\boldsymbol{F}\left(\mathbb{\Omega}_{0}\right)$. Since $\boldsymbol{U}(\mathfrak{D} \mathfrak{B} ; 2)$ is trivial, $|\mathfrak{F}|$ is odd. Since $\mathfrak{F}$ is a 3 '-group, and since $\sigma_{2}=\{3\}$, it follows that $C_{\S}(I)$ is cyclic for every element $I$ of $\mathfrak{S}_{\tilde{2}}^{\sharp}$. Thus if $I \in \boldsymbol{Z}\left(\mathfrak{S}_{0}\right)$, then $\mathfrak{S}_{0}^{\prime}$ centralizes $\boldsymbol{C}_{\widetilde{\mho}}(I)$. Since $\mathfrak{F}=\left\langle\boldsymbol{C}_{\mathfrak{F}}(I) \mid I \in \boldsymbol{Z}\left(\mathfrak{F}_{0}\right)^{\sharp}\right\rangle$, it follows that $\mathfrak{S}_{0}^{\prime}$ centralizes $\mathfrak{F}$, so $\mathfrak{S}_{0}^{\prime} \subseteq \mathfrak{F}$. Since $|\mathfrak{F}|$ is odd, we conclude that $C_{\mathfrak{p}}(B)$ is abelian for every $B$ in $\mathfrak{B}^{\sharp}$. Thus, for each $B$ in $\mathfrak{S}^{\sharp}$, either $C_{\mathfrak{\xi}}(B)=1$ or $C_{\mathfrak{\xi}}(B)$ is homocyclic on 2 generators.

Suppose the lemma is false. Since $\mathfrak{S}=\left\langle C_{\mathfrak{F}}(B) \mid B \in \mathfrak{B}^{\ddagger}\right\rangle$, it fol- 
lows that for some $B$ in $\mathfrak{B}^{\sharp}, C_{\mathfrak{p}}(B)$ is not permutable with $\mathfrak{D}$. Let $\mathfrak{R}=C(B), \mathbb{\Omega}_{0}=\boldsymbol{O}_{3^{\prime}}(\mathfrak{R})$. Thus, by Lemma $14.7, \mathfrak{D} \subseteq \mathfrak{\Omega}_{0}$ and $C_{\mathfrak{\xi}}(B) \subseteq \mathfrak{\Omega}_{0}$. Let $\mathfrak{S}_{0}=C_{\mathfrak{f}}(B)$. Let $\mathfrak{S}_{1}$ be a $S_{2}$-subgroup of $\mathfrak{R}_{0}$ which admits $\mathfrak{B}$ and contains $\mathfrak{S}_{0}$. By Lemma 6.2 , there is $C$ in $\boldsymbol{C}(\mathfrak{B})$ such that $\mathfrak{S}_{1}^{c} \subseteq \mathfrak{F}_{2}$. Since $C(\mathfrak{B}) \subseteq \mathbb{R}$, it follows that $\mathfrak{S}_{1}^{C} \subseteq \mathfrak{R}_{0}$, so $\mathfrak{S}_{1}^{C} \subseteq \mathfrak{R}_{0} \cap \mathfrak{S}_{\mathcal{E}}=\mathfrak{S}_{0}$. This implies that $\mathscr{Y}_{0}$ is a $S_{2}$-subgroup of $\Omega_{0}$.

Let $\mathbb{R}_{1}$ be a $S_{2}$-subgroup of $\mathbb{Z}_{0}$ which contains $\mathfrak{D}$. Since $\mathbb{R}_{1}$ and $\mathfrak{S}_{0}$ are permutable, it follows that $\mathfrak{D} \subset \mathbb{R}_{1}$. Let $q \in \pi\left(\mathfrak{R}_{1}\right)$ be chosen so that a. $S_{p}$-subgroup of $\mathfrak{R}_{1}$ is permutable with $\mathfrak{S}_{0}$, admits $\mathfrak{B}$, and is not contained in $\mathfrak{D}$. Let $\mathfrak{F}=\mathfrak{S}_{0} \mathfrak{O} \mathfrak{O}$. By Lemma 14.11, $\boldsymbol{O}_{2}(\mathfrak{F})=1$. Since $q \geqslant 5$, and since $\mathfrak{S}_{0}$ is abelian with $m\left(\mathfrak{S}_{0}\right)=2$, and since $\mathfrak{B}$ is elementary of order 9 , it follows that $\mathfrak{\checkmark} \checkmark$.

Since $\sigma_{2}=\{3\}$, it follows that $C_{\Omega}(H)$ is cyclic for every involution $H$ of $\mathscr{S}_{0}$. Since $\mathfrak{S}_{0}$ is represented faithfully on $\mathfrak{Q}$, it follows that $C_{\mathbb{0}}\left(\mathfrak{S}_{0}\right)=1$. Let $\mathfrak{B}$ be a minimal normal subgroup of $\mathfrak{F}$ with $\mathfrak{B} \subseteq \mathfrak{D}$. Then $\mathfrak{B}$ is elementary of order $q^{3}$. In particular, $q \in \pi_{3} \cup \pi_{4}$. By Theorems 10.2 and 10.8, it follows that $\mathscr{A}(q) \subseteq \mathscr{C}^{*}(\mathbb{S})$. Let $\mathfrak{S}=M(\mathfrak{F})$, and let $\Re$ be a $S_{2,3, q}$-subgroup of $\subseteq$ which contains $\mathfrak{F}$. Let $\left\{\Re_{2}, \Re_{3}, \Re_{q}\right\}$ be a Sylow system of $\Re$ with $\mathfrak{S}_{0} \subseteq \Re_{2}, \mathfrak{B} \subseteq \Re_{3}, \mathfrak{Q} \subseteq \Re_{q}$. Since $3 \in \sigma_{2}, \Re_{3}$ normalizes $\Re_{q}$. By Lemma 14.7, $\mathfrak{S}_{0} \subseteq \boldsymbol{O}_{2}\left(\Re_{2} \Re_{3}\right)$. If the containment is proper, let $\tilde{\mathfrak{S}}_{0} / \mathscr{S}_{0}$ be a chief factor of $\boldsymbol{O}_{2}\left(\Re_{2} \Re_{3}\right) \mathfrak{B}$ with $\tilde{\mathcal{H}}_{0} \subseteq \boldsymbol{O}_{2}\left(\Re_{2} \Re_{3}\right)$. Then $\mathscr{S}_{0}=C_{\tilde{\mathfrak{F}}_{0}}(B)$ and $\tilde{\mathfrak{F}}_{0} / \mathscr{S}_{0}$ is a four-group. This implies that $\tilde{\mathfrak{K}}_{0}$ is the direct product of $\mathfrak{S}_{0}$ and a four-group, since $\mathfrak{B}$ acts faithfully on $\tilde{\mathcal{F}}_{0}$. But $\boldsymbol{O}_{2}(\Re)=1$, so $\tilde{\mathcal{F}}_{0}$ is represented faithfully on $\boldsymbol{O}_{q}(\Re)$. This implies that $q \in \sigma_{2}$. This contradiction yields that $\mathfrak{S}_{0}=\boldsymbol{O}_{2}\left(\Re_{2} \Re_{3}\right)$. Hence, $\boldsymbol{O}_{3^{\prime}}(\Re)=\mathfrak{S}_{0} \Re_{q}$, so that $\Re_{q} \operatorname{char} \boldsymbol{O}_{3^{\prime}}(\Re) \triangleleft \Re$.

Since $\boldsymbol{C}_{\mathrm{R}_{p}}(H)$ is cyclic for all involutions $H$ of $\mathfrak{S}_{0}$, it follows that $\boldsymbol{C}_{\mathfrak{\Omega}_{q}}\left(\mathfrak{S}_{0}\right)=1$. Let $\mathfrak{X}=\Omega_{1}\left(\boldsymbol{Z}\left(\Re_{q}\right)\right)$. Thus, $\mathfrak{X}$ is elementary of order $q^{3}$ and is an irreducible $\mathfrak{S}_{0} \mathfrak{B}$-group. We argue that $\mathfrak{X}=\mathfrak{W}$. Otherwise, $\langle\mathfrak{X}, \mathfrak{B}\rangle$ is elementary of order $q^{6}$, so that $q \in \sigma_{2}$. Hence, $\mathfrak{X}=\mathfrak{W}$.

We next argue that $B$ centralizes $\Re_{q}$. Suppose false. Let $\tilde{\Omega}_{q}=$ $\mathfrak{\Re}_{q} \cap \boldsymbol{C}(B)$. Thus, $\tilde{\Re}_{q}$ admits $\mathfrak{K}_{0} \mathfrak{B}$ and $\tilde{\Re}_{q} \subset \Re_{q}$. Let $\mathfrak{Y}$ be a subgroup of $\Re_{q}$ which admits $\mathfrak{S}_{0} \mathfrak{B}$ and is minimal subject to $[\mathfrak{Y}, B] \neq 1$. Then, $B$ centralizes $\boldsymbol{D}(\mathfrak{Y})$, and $[\mathfrak{Y},\langle B\rangle]=\mathfrak{Y}$. Hence, $\boldsymbol{D}(\mathfrak{Y}) \leqq \boldsymbol{Z}(\mathfrak{Y})$, by Lemma 0.8.5. By 0.3.6, we get that $\mathfrak{Y}$ is of exponent $q$. If $|\mathfrak{Y}|>q^{3}$, then $q \in \sigma_{2}$. Hence, $\mathfrak{Y}$ is elementary of order $q^{3}$. This implies that $\mathfrak{Y X}$ is elementary of order $q^{6}$, so again we get $q \in \sigma_{2}$. This contradiction forces $\Re_{q} \subseteq C(B)$.

Set $\langle B\rangle=\mathfrak{B}_{0}$, and let $\mathfrak{B}_{1}, \mathfrak{B}_{2}, \mathfrak{B}_{3}$ be the remaining subgroups of $\mathfrak{B}$ of order 3 . Since $\mathfrak{S}_{0} \mathfrak{B}_{i}$ is a Frobenius group, it follows that $C_{\mathfrak{R}_{q}}\left(\mathfrak{B}_{i}\right) \neq 1, i=1,2,3$. By Lemma 6.2, it follows that

$$
N(\mathfrak{B})=N_{\odot}(\mathfrak{B}) \cdot C(\mathfrak{B}) \text {. }
$$

Since $\mathfrak{B}_{0}=\boldsymbol{C}_{\mathfrak{P}}\left(\boldsymbol{O}_{q^{\prime}, q}(\mathfrak{S}) / \boldsymbol{O}_{q^{\prime}}(\mathfrak{S})\right)$, it follows that $\mathfrak{B}_{0} \triangleleft N(\mathfrak{B})$. 
Now $\mathfrak{B}$ acts faithfully on $\mathscr{S}$, so $\mathfrak{S}_{0} \subset \mathfrak{F}$. Hence, for some $i \in\{1,2,3\}, C_{\mathfrak{s}}\left(\mathfrak{B}_{i}\right) \neq 1$. We may assume notation is chosen so that $\boldsymbol{C}_{\mathfrak{5}}\left(\mathfrak{B}_{1}\right) \neq 1$. Set $\mathfrak{S}_{1}=\boldsymbol{C}_{\mathfrak{F}}\left(\mathfrak{B}_{1}\right)$, so that $\mathfrak{F}_{1}$ is a homocyclic abelian group with $m\left(\mathfrak{H}_{1}\right)=2$. Let $\mathfrak{R}_{1}=C\left(\mathfrak{B}_{1}\right)$ and let $\widetilde{\mathfrak{R}}_{1}$ be a $S_{2,3, q}$-subgroup of $\mathfrak{Q}_{1}$ which contains $\boldsymbol{C}_{\mathfrak{\Omega}_{q}}\left(\mathfrak{B}_{1}\right) \mathfrak{B}$. Let $\mathfrak{F}_{1}=\boldsymbol{O}_{3^{\prime}}\left(\tilde{\mathfrak{R}}_{1}\right)$. By Lemma 14.7, $\mathfrak{Q}_{1} \subseteq \mathfrak{F}_{1}$, where $\mathfrak{Q}_{1}=C_{\mathfrak{S}_{q}}\left(\mathfrak{B}_{1}\right)$. As we have seen, $\mathfrak{Q}_{1} \neq 1$. Also, $\mathfrak{S}_{1}^{C}$ is a $S_{2}$-subgroup of $\mathfrak{F}_{1}$, by Lemma 6.2 , where $C$ is a suitable element of $C(\mathfrak{B})$. Since $m\left(\mathfrak{S}_{1}\right)=2$, and $\mathfrak{S}_{1}^{\prime}=1$, it follows that $\mathfrak{F}_{1}$ is $q$-closed. Since $\mathfrak{F}_{1} \cap \Re_{q} \neq 1$, it follows that $\boldsymbol{O}_{q}\left(\mathfrak{F}_{1}\right) \subseteq \mathfrak{S}^{*}$, since $\mathscr{A}(q) \subseteq \mathscr{M}^{*}(\mathbb{S})$ and $C(X)$ contains an elementary subgroup of $\Re_{q}$ of order $q^{3}$ for every $X$ in $\Re_{q}$. Also, since $\mathscr{A}(q) \subseteq \mathscr{H}^{*}(\mathbb{S}), \quad N\left(\boldsymbol{O}_{q}\left(\mathfrak{F}_{1}\right)\right) \leqq \mathfrak{S}$, so $\mathfrak{H}_{1}^{C} \subseteq \mathfrak{S}$. Since $\mathfrak{S}_{1}^{C} \in \boldsymbol{U}(\mathfrak{B} ; 2)$, Lemma 14.7 implies that $\mathfrak{S}_{1}^{C} \subseteq \boldsymbol{O}_{3^{\prime}}(\mathfrak{S})$. Since $\mathfrak{S}_{0}$ is a $S_{2}$-subgroup of $\boldsymbol{O}_{3^{\prime}}(\mathfrak{S})$, it follows that for some $D$ in $\boldsymbol{C}(\mathfrak{B}), \mathfrak{S}_{1}^{C D} \subseteq \mathfrak{S}_{0}$. This is absurd, since $\mathfrak{B}_{1}$ centralizes $\mathfrak{S}_{1}^{C D}$, while $\mathfrak{S}_{0} \mathfrak{B}_{1}$ is a Frobenius group. The proof is complete.

Lemma 14.16. With the preceding notation, set $\mathfrak{L}=\mathfrak{S} \cdot \boldsymbol{O}_{r^{\prime}}(\boldsymbol{C}(\mathfrak{B}))$, $\mathfrak{M}=N(\mathbb{R})$. Then $\mathfrak{M}$ is the unique element of $\mathscr{M S}(\mathbb{S})$ which contains $\mathfrak{B} \mathbb{R}$ and $\mathbb{L}=\boldsymbol{O}_{r}(\mathfrak{M})$.

Proof. By Lemma 14.15, $\&$ is a subgroup of 89 . We will show that $\mathbb{R}$ is solvable. Let $3=Z(\mathscr{Y})$. We can then choose $B$ in $\mathfrak{B}^{\sharp}$ such that $\mathfrak{B}_{0}=C_{3}(B) \neq 1$. Let $\mathfrak{R}_{1}=C_{\varepsilon}(B)=\boldsymbol{O}_{r^{\prime}}(C(B)) \cdot C_{\mathfrak{\xi}}(B)$. Thus, $\mathfrak{Q}_{1}$ is solvable, and

$$
\bigcap_{x \in \mathfrak{Q}} \mathfrak{Q}_{1}^{x}=\bigcap_{x \in \mathfrak{\mathfrak { S }}} \mathfrak{Q}_{1}^{x}=\Re \supseteqq 3_{0} \neq 1 \text {. }
$$

Thus, $\mathbb{Z} \subseteq N(\Re)$. Since $(\mathcal{S}$ is an $N$-group, $N(\Re)$ is solvable. So $\&$ is solvable.

Since $\mathbb{R}$ is solvable, $\mathfrak{M}$ is solvable.

Suppose $\subseteq \in \operatorname{Sol}(\mathbb{S}), \mathbb{R B} \cong \subseteq$. By Lemma 14.7, $\mathbb{R} \subseteq \boldsymbol{O}_{r^{\prime}}(\mathfrak{S}) . \quad$ By maximality of $\mathfrak{S}$, $\mathfrak{S}$ is a $S_{2}$-subgroup of $\boldsymbol{O}_{r^{\prime}}(\mathfrak{S})$. Choose

$$
q \in \pi\left(\boldsymbol{O}_{r^{\prime}}(\mathfrak{S})\right)-\{2\}
$$

and let $\cong$ be a $S_{q}$-subgroup of $\boldsymbol{O}_{r^{\prime}}(\mathfrak{S})$ which admits $\mathfrak{B}$ and is permutable with $\mathfrak{S}$. Suppose $[\mathfrak{O}, \mathfrak{B}] \neq 1$. By Lemma 14.13, we get

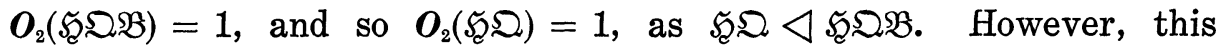
violates Lemma 14.12. We conclude that $[\mathfrak{Q}, \mathfrak{B}]=1$. This implies that $\boldsymbol{O}_{r^{\prime}}(\boldsymbol{C}(\mathfrak{B}))$ contains a $S_{2^{\prime}}$-subgroup of $\boldsymbol{O}_{r^{\prime}}(\mathfrak{S})$, and so $\boldsymbol{O}_{r^{\prime}}(\mathfrak{S})=$ $\mathfrak{S} \circlearrowleft$. This completes the proof.

We now indicate explicitly the dependence of $\mathfrak{M}$ on the various subgroups in question. Let $\mathfrak{B}$ be an elementary subgroup of $\mathfrak{R}$ of order $r^{2}$. Then let $\mathfrak{S}$ be a maximal element of $И(\Re ; 2)$. Then set $\mathfrak{M}=\mathfrak{M}(\mathfrak{B}, \mathfrak{S}, \mathfrak{R})=\boldsymbol{N}\left(\mathfrak{S} \cdot \boldsymbol{O}_{r^{\prime}}(\boldsymbol{C}(\mathfrak{B}))\right)$. 
LEMma 14.17. $\quad C(\mathfrak{B}) \subseteq \mathfrak{M}(\mathfrak{B}, \mathfrak{F}, \mathfrak{R})$ for all relevant $\mathfrak{S}, \mathfrak{R}, \mathfrak{B}$.

Proof. By Lemma 14.14, C(⿻上) is $r^{\prime}$-closed. Let $\mathfrak{M}=\mathfrak{M}(\mathfrak{B}, \mathfrak{S}, \mathfrak{R})$. Since $\mathfrak{M} \supseteqq \boldsymbol{O}_{r^{\prime}}(\boldsymbol{C}(\mathfrak{B}))$, it suffices to show that $\mathfrak{M}$ contains a $S_{r}$-subgroup of $\boldsymbol{C}(\mathfrak{B})$.

Case 1. There is a $S_{r}$-subgroup of $\Re_{0}$ of $\boldsymbol{C}(\mathfrak{B})$ which normalizes $\mathfrak{F}$.

Since $\mathfrak{R}_{0}$ normalizes $\mathfrak{S}$ and $\boldsymbol{O}_{r^{\prime}}(\boldsymbol{C}(\mathfrak{B}))$, we have $\mathfrak{R}_{0} \subseteq \mathfrak{M}$, and we are done.

Case 2. $N(\mathfrak{E})$ contains no $S_{r}$-subgroup of $C(\mathfrak{B})$.

Since $\Re$ is a $S_{r}$-subgroup of $(B)$, we have $\Re^{\prime} \neq 1$. By Lemma 14.1, $\boldsymbol{Z}(\mathfrak{R})$ is cyclic. Since $r \in \pi_{2}, \mathfrak{B}$ char $\boldsymbol{C}_{\mathfrak{r}}(\mathfrak{B})$, as $\mathfrak{B}=\Omega_{1}\left(\boldsymbol{C}_{\mathfrak{r}}(\mathfrak{B})\right)$. Hence, $\boldsymbol{N}_{\mathfrak{R}}(\mathfrak{B}) \supset \boldsymbol{C}_{\mathfrak{r}}(\mathfrak{B})$, and so $\left|\mathfrak{B}^{*}\right| \geqslant r^{2}-r$, where

$$
\mathfrak{B}^{*}=\left\{B \mid B \in \mathfrak{B}^{\sharp}, C_{\mathfrak{\wp}}(B) \neq 1\right\} \text {. }
$$

Let $\Re$ be a $S_{r}$-subgroup of $C(\mathfrak{B})$. Choose $G$ in $(5)$ such that $\tilde{\mathfrak{R}} \subseteq \mathfrak{R}^{G}$. Thus, $\mathfrak{B}$ normalizes $\mathfrak{S}^{G}$. Since $\boldsymbol{N}_{\mathfrak{R}}$. $(\mathfrak{B}) \supset C_{\mathfrak{R}} G(\mathfrak{B})$, it follows that $\left|\mathfrak{B}^{* *}\right| \geqslant r^{2}-r$, where $\mathfrak{B}^{* *}=\left\{B \mid B \in \mathfrak{B}^{\sharp}, C_{\mathfrak{F}^{G}}(B) \neq 1\right\}$. Hence, $\mathfrak{B}^{*} \cap \mathfrak{B}^{* *} \neq \varnothing$. By Lemma 6.2, we get $\mathfrak{S}=\mathfrak{S}^{G C}$ for some $C$ in $C(\mathfrak{B})$. Thus, $\tilde{\mathfrak{R}}$ normalizes $\mathfrak{S}^{G}$, and $\tilde{\mathfrak{R}}^{c}$ normalizes $\mathfrak{S}^{G C}=\mathfrak{S}$. Since $\tilde{\mathfrak{R}}^{c}$ is a $S_{r}$-subgroup of $\boldsymbol{C}(\mathfrak{B})$, we see that this case does not occur. The proof is complete.

Lemma 14.18. Suppose $\mathfrak{B}_{1}, \mathfrak{B}_{2}$ are elementary subgroups of $\mathfrak{R}$ of order $r^{2}$. Then $\mathfrak{M}\left(\mathfrak{B}_{1}, \mathfrak{F}, \mathfrak{R}\right)=\mathfrak{M}\left(\mathfrak{B}_{2}, \mathfrak{K}, \mathfrak{R}\right)$.

Proof. We may assume that $\mathfrak{B}_{1} \neq \mathfrak{B}_{2}$. Hence, $\mathfrak{R}^{\prime} \neq 1$, since $r \in \pi_{2}$. Choose $\mathfrak{U} \in \mathscr{C}(\mathfrak{R})$. We assume without loss of generality that $\mathfrak{B}_{1}=\mathfrak{U}$.

Let $\Re_{0}=\mathfrak{B}_{1} \mathfrak{B}_{2}$, a non abelian group of order $r^{3}$ and exponent $r$. Let $\Re_{1}, \Re_{2}$ be distinct subgroups of $\Re_{0}$ of order $r^{2}$. Let $\mathfrak{D}_{i}$ be a $S_{2^{\prime}}$ subgroup of $\boldsymbol{O}_{r^{\prime}}\left(\boldsymbol{C}\left(\Re_{i}\right)\right)$ which admits $\Re_{0}, i=1,2$; $\mathfrak{D}_{i}$ exists since $\boldsymbol{O}_{r^{\prime}}\left(C\left(\Re_{i}\right)\right) \triangleleft N\left(\Re_{i}\right)$ and $\Re_{0} \subseteq N\left(\Re_{i}\right)$. Then $\mathfrak{S C D}_{i}=\mathfrak{S C}_{r^{\prime}}\left(C\left(\Re_{i}\right)\right), i=1,2$. Suppose we can show that $\Re_{2}$ centralizes $\mathfrak{D}_{1}$. In this case, we get $\mathfrak{S C}_{2} \supset \mathfrak{D}_{1}$, so by symmetry, $\left|\mathfrak{D}_{2}\right|=\left|\mathfrak{D}_{1}\right|$, so that $\mathfrak{S C D}_{1}=\mathfrak{S}_{\mathfrak{C}} \mathfrak{D}_{2}$. Thus, it suffices to show that $\Re_{2}$ centralizes $\mathfrak{D}_{1}$.

Since $\mathfrak{S C D}_{1}$ is a solvable group which admits $\Re_{0}$, for each prime $q \in \pi\left(\mathfrak{D}_{1}\right)$, there is a $S_{p}$-subgroup $\mathfrak{D}$ of $\mathfrak{D}_{1}$ which admits $\Re_{0}$ and is permutable with $\mathfrak{S}_{\text {. }}$ Suppose by way of contradiction that $\left[\Re_{0}, \mathfrak{\Omega}\right] \neq 1$.

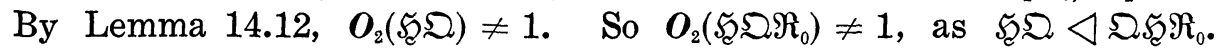
This violates Lemma 14.13. The proof is complete. 
We may now write $\mathfrak{M}(\mathfrak{B}, \mathfrak{F}, \mathfrak{R})=\mathfrak{M}(\mathfrak{F}, \mathfrak{R})$, without ambiguity.

LEMMA 14.19. $\quad N(\mathfrak{S}) \subseteq \mathfrak{M}(\mathfrak{F}, \mathfrak{R})$.

Proof. Set $\mathfrak{M}=\mathfrak{M}(\mathfrak{S}, \mathfrak{R}), \mathfrak{R}=N(\mathfrak{S}), N_{0}=\boldsymbol{O}_{r^{\prime}}(\mathfrak{R})$. Since $\mathfrak{S}$ is a maximal element of $И(\Re ; 2), \mathfrak{F}$ is a $S_{2}$-subgroup of $\mathfrak{N}_{0}$. Let $\mathfrak{B}$ be an elementary subgroup of $\Re$ of order $r^{2}$, and let $\mathfrak{D}_{0}$ be a $S_{2}$-subgroup of $\mathfrak{N}_{0}$ which admits $\mathfrak{B}$. By Lemmas 14.12 and 14.13 , $\mathfrak{B}$ centralizes $\mathfrak{D}_{0}$, so $\mathfrak{D}_{0} \subseteq \mathfrak{S} \cdot \boldsymbol{O}_{r^{\prime}}(\boldsymbol{C}(\mathfrak{B})) \subseteq \mathfrak{M}$.

Case 1. $\Re^{\prime}=1$.

Here we get $\mathfrak{N}_{0} \mathfrak{B} \triangleleft \mathfrak{N}$, so it suffices to show that $N_{\mathfrak{R}}(\mathfrak{B}) \subseteq \mathfrak{M}$. Since $N_{\mathfrak{R}}(\mathfrak{B})$ normalizes $C(\mathfrak{B})$, it follows that $N_{\mathfrak{r}}(\mathfrak{B})$ normalizes $\mathfrak{S}$ and $\boldsymbol{O}_{r^{\prime}}(C(\mathfrak{B}))$, so $\boldsymbol{N}_{\mathfrak{R}}(\mathfrak{B}) \subseteq \mathfrak{M}$.

Case 2. $\Re^{\prime} \neq 1$.

Let $\Re_{0}=\mathfrak{R} \cap \boldsymbol{O}_{r^{\prime}, r}(\mathfrak{R})$, so that $\boldsymbol{O}_{r^{\prime}, r}(\mathfrak{R})=\mathfrak{N}_{0} \Re_{0}$. First, suppose that $\Re_{0}$ is not of symplectic type. Let $\mathfrak{U}$ be a non cyclic characteristic elementary subgroup of $\mathfrak{R}_{0}$. Thus, $\mathfrak{R}=\mathfrak{N}_{0} \boldsymbol{N}_{\mathfrak{R}}(\mathfrak{U})$. Since $\boldsymbol{O}_{r^{\prime}}(\mathfrak{M})=$ $\mathfrak{S} \cdot \boldsymbol{O}_{r^{\prime}}(\boldsymbol{C}(\mathfrak{H}))$, we get $\boldsymbol{N}_{\mathfrak{n}}(\mathfrak{U}) \subseteq \mathfrak{M}$. We may therefore assume that $\Re_{0}$ is of symplectic type. Let $\Re^{*}=\Omega_{1}\left(\Re_{0}\right)$. By Lemma 14.17,

$$
\boldsymbol{O}_{r^{\prime}}(\mathfrak{M})=\mathfrak{S E}_{\boldsymbol{r}^{\prime}}\left(\boldsymbol{C}\left(\mathfrak{R}^{*}\right)\right),
$$

so $N_{\mathfrak{R}}\left(\Re^{*}\right) \subseteq \mathfrak{M}$. Since $\mathfrak{M}=\mathfrak{R}_{0} N\left(\Re^{*}\right)$, the proof is complete.

Lemma 14.20. For each elementary subgroup $\mathfrak{B}$ of $\mathfrak{R}$ of order $r^{2}$, $\boldsymbol{O}_{2}(\mathfrak{M}) \mathfrak{B} \in \mathscr{C l} *(\mathfrak{S})$, where $\mathfrak{M}=\mathfrak{M}(\mathfrak{\mathcal { B }}, \mathfrak{R})$.

Proof. Set $\mathfrak{S}_{0}=\boldsymbol{O}_{2}(\mathfrak{M})$. We first show that $\mathfrak{B}$ centralizes $\mathfrak{S C}_{\mathcal{C}} / \mathfrak{S}_{0}$. Let $\mathfrak{Z}=\boldsymbol{O}_{r^{\prime}}(\mathfrak{M})$, so that $\mathfrak{Z}=\mathfrak{S C O}_{r^{\prime}}(\boldsymbol{C}(\mathfrak{B}))$. Hence, $\mathfrak{B}$ centralizes

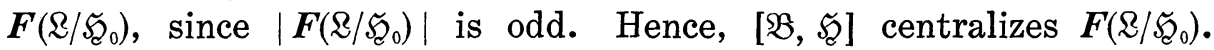

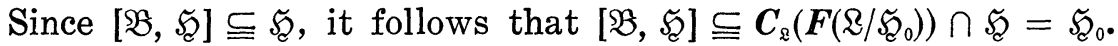

Since $[\mathfrak{S}, \mathfrak{B}] \subseteq \mathfrak{F}_{0}$, and since $\mathfrak{B}$ is represented faithfully on $\mathfrak{K}$, $\mathfrak{B}$ is represented faithfully on $\mathfrak{S}_{0}$. In particular, $\mathfrak{S}_{0} \neq 1$.

Let $\mathscr{S}=\left\{\mathfrak{S} \mid \mathfrak{S} \in \mathscr{S}\right.$ ol $\left.(\mathbb{S}), \mathscr{S}_{0} \mathfrak{B} \subseteq \subseteq \subseteq \mathfrak{M}\right\}$. Suppose by way of contradiction that $\mathscr{S} \neq \varnothing$. Choose $\mathfrak{S}$ in $\mathscr{S}$ such that $|\mathfrak{S} \cap \mathbb{R}|_{2}$ is maximal. Set $\mathfrak{S}_{0}=\boldsymbol{O}_{r^{\prime}}(\mathfrak{S})$. Since $\mathfrak{S} \cap \mathbb{R} \in \boldsymbol{U}\left(\mathfrak{B} ; r^{\prime}\right)$, we get $\mathfrak{S} \cap \mathbb{L}=\mathfrak{S}_{0} \cap \mathbb{L} \supseteqq \mathfrak{F}_{0}$. Let $\mathfrak{S}_{1}$ be a $S_{2}$-subgroup of $\mathfrak{S}_{0} \cap \mathbb{R}$ which contains $\mathfrak{F}_{0}$ and admits $\mathfrak{B}$, and let $\mathfrak{S}_{2}$ be a $S_{2}$-subgroup of $\mathfrak{S}_{0}$ which contains $\mathfrak{S}_{1}$ and admits $\mathfrak{B}$. Suppose $\mathfrak{S}_{1} \subset \mathfrak{S}_{2}$. Since $\mathfrak{S}$ is a maximal element of $И(\mathfrak{B} ; 2)$, it follows that $\mathfrak{S}_{0} \subset \mathfrak{S}_{\mathcal{C}}$. Since $\mathfrak{B}$ centralizes $\mathfrak{S}_{\mathfrak{C}} / \mathfrak{S}_{0}$, we conclude that $\boldsymbol{C}_{\mathfrak{F}}(\mathfrak{B}) \neq 1$. 
By Lemma 6.2, $\mathfrak{S C}_{2} \subseteq \mathfrak{F}^{C}$ for some $C$ in $C(\mathfrak{B})$. Hence, $\mathfrak{S}_{2} \subseteq \mathbb{R}$, so $\mathfrak{S}_{2} \subseteq \mathfrak{R} \cap \mathfrak{S}=\mathbb{R} \cap \mathfrak{S}_{0}$, against $\left|\mathfrak{S}_{2}\right|>\left|\mathfrak{S}_{1}\right|=\left|\mathfrak{R} \cap \mathfrak{S}_{0}\right|_{2}$. We conclude that $\mathfrak{S}_{1}=\mathfrak{S}_{2}$.

We next argue that $N\left(\mathfrak{S}_{1}\right) \subseteq \mathfrak{M}$. This is clear if $\mathfrak{S}_{1}$ is a $S_{2}$-subgroup of $\mathbb{R}$, by Lemma 14.18. Otherwise, $\left|N\left(\mathfrak{S}_{1}\right) \cap \&\right|_{2}>|\subseteq \cap \&|_{2}$, so by maximality of $|\mathfrak{S} \cap \mathbb{R}|_{2}$, we get $N\left(\mathfrak{S}_{1}\right) \subseteq \mathfrak{M}$. Since $\mathfrak{S}=\mathfrak{S}_{0} \boldsymbol{N}_{\mathfrak{E}}\left(\mathfrak{S}_{\mathcal{E}}\right)$, it follows that $\mathfrak{S}_{0} \nsubseteq \mathfrak{M}$.

Since $\mathfrak{S}_{1} \subseteq \mathfrak{M}$ and $\mathfrak{S}_{0} \nsubseteq \mathfrak{M}$, it follows that for some odd prime $q$, $\mathfrak{S}_{0}$ contains a $S_{q}$-subgroup $\mathfrak{S}_{q}$ which is normalized by $\mathfrak{B}$, is permutable with $\mathfrak{S}_{1}$ and satisfies $\mathfrak{S}_{q} \nsubseteq \mathfrak{M}$. By Lemma $14.13, \boldsymbol{O}_{2}\left(\mathfrak{S}_{1} \mathfrak{S}_{q}\right)=1$, since $\boldsymbol{O}_{2}\left(\mathfrak{S}_{1} \mathfrak{S}_{q}\right) \in \boldsymbol{U}\left(\mathfrak{S}_{q} \mathfrak{B} ; 2\right)$.

\section{Case 1. $\quad r=3$.}

Since $q \neq 3$, it follows that $q \in \sigma_{0} \cup \sigma_{1}$. Since $\mathscr{F}_{1}$ is represented faithfully on $\mathfrak{Q}=\boldsymbol{O}_{q}\left(\mathfrak{S}_{1} \mathfrak{S}_{q}\right)$, it follows that $\boldsymbol{C}_{凤}(I)$ is cyclic for all involutions $I$ of $\mathfrak{K}_{1}$. Hence, $\mathfrak{S}_{1}$ contains no elementary subgroup of order 8 , by Lemma 5.34. On the other hand, $\mathfrak{B}$ is represented faithfully on $\mathfrak{F}_{1}$, so $\mathfrak{H}_{1}$ contains a four-group $\mathfrak{B}$. Since $C_{\mathfrak{o}}(V)$ is cyclic for all $V$ in $\mathfrak{B}^{\sharp}$, it follows that $m(\mathfrak{\Omega}) \leqq 3$. Thus, $\mathfrak{S}_{1} \mathfrak{B}$ is isomorphic to a subgroup of $G L(3, q)$. This is not the case, since $\mathfrak{B}$ is represented faithfully on $\mathfrak{S}_{1}$.

\section{Case 2. $r \neq 3$.}

Choose $B$ in $\mathfrak{B}^{*}$ such that $B$ does not centralize $C_{\mathfrak{w}_{1}}(B)$. Since $r \geqslant 5$, it follows that $C_{\mathfrak{q}_{1}}(B)$ contains a four-group $\mathfrak{F}$. Let $\mathfrak{\Omega}_{0}$ be a subgroup of $\mathfrak{Q}$ which admits $\langle\mathfrak{F}, B\rangle$ and is minimal subject to $\left[\mathfrak{Q}_{0}, B\right] \neq 1$. The minimality of $\mathfrak{\Omega}_{0}$ guarantees that $\mathfrak{\Omega}_{0}$ is of exponent $q$. We argue that $q>r$. Otherwise, we get $m\left(\Omega_{0}\right) \geqslant 3$. Since $\mathfrak{\Omega}_{0}=$ $C_{\bigotimes_{0}}(E)$ for some $E$ in $\xi^{*}$, this forces $q \notin \sigma_{0} \cup \sigma_{1} \cup \sigma_{2}$. Hence, $q>r$.

By maximality of $r$, it follows that $q \in \sigma_{0} \cup \sigma_{1}$. Hence, $C_{20}(E)$ is cyclic for all $E$ in $\left(F^{\#}\right.$. Hence, $m(\mathfrak{Q}) \leqq 3$, and $\mathfrak{F}_{1} \mathfrak{B}$ is isomorphic to a subgroup of $G L(3, q)$. This is not the case, since $\mathfrak{B}$ is represented faithfully on $\mathfrak{S}_{1}$. The proof is complete.

LEMma 14.21. For each elementary subgroup $\mathfrak{B}$ of $\mathfrak{R}$ of order $r^{2}$, one of the following holds:

(a) $\mathfrak{B} \in \mathscr{M}^{*}(\mathbb{S})$.

(b) $\boldsymbol{C}_{\mathfrak{5}}(\mathfrak{B})$ contains no four-group.

Proof. Suppose $\mathfrak{B}$ is a four-group contained in $C(\mathfrak{B})$. Choose $B \in \mathfrak{B}^{\sharp}$ and set $\mathfrak{E}=\boldsymbol{C}(B) \supseteqq \mathfrak{B} \mathfrak{B}=\mathfrak{B} \times \mathfrak{B}$. Let $\mathfrak{F}_{0}=\boldsymbol{O}_{r^{\prime}}(\mathfrak{E}) . \quad$ By Lemma 14.7, $\mathbb{E}_{0}$ contains every element of $\boldsymbol{И}_{\mathbb{\mho}}\left(\mathfrak{B} ; r^{\prime}\right)$. In particular, 
$\mathfrak{B} \subseteq \mathfrak{S}_{0}$. Let $q$ be an odd prime in $\pi\left(\mathbb{E}_{0}\right)$, and let $\mathfrak{\Im}_{1}$ be a $S_{2, q}$-subgroup of $\mathfrak{F}_{0}$ which contains $\mathfrak{B}$ and admits $\mathfrak{B}$. Let $\left\{\mathfrak{F}_{2}, \mathfrak{F}_{q}\right\}$ be a Sylow system of $\mathfrak{F}_{1}$ which admits $\mathfrak{B}$ and satisfies $\mathfrak{B} \subseteq \mathfrak{E}_{2}$. Suppose by way of contradiction that $\left[\mathfrak{B}, \widetilde{C}_{q}\right] \neq 1$. By Lemma $14.13, И\left(\mathfrak{B}_{2} ; 2\right)$ is trivial. Hence, $\mathfrak{B}$ is represented faithfully on $\boldsymbol{O}_{q}\left(\mathfrak{B} \mathfrak{C}_{1}\right)$, and $\mathfrak{B}$ is represented non trivially on $\mathfrak{Q}_{q}\left(\mathfrak{B C}_{1}\right)$. Choose $V$ in $\mathfrak{B}^{\sharp}$ such that $\mathfrak{B}$ does not centralize $\boldsymbol{O}_{q}\left(\mathfrak{B} \mathfrak{C}_{1}\right) \cap \boldsymbol{C}(V)=\mathfrak{\Omega}$, say. Thus, $\langle V\rangle \in \boldsymbol{И}(\mathfrak{B} \Omega$; 2), against Lemma 4.13. This contradiction implies that $\mathfrak{B}$ centralizes every element of $\boldsymbol{U}_{\mathbb{E}}\left(\mathfrak{B} ;\{2, r\}^{\prime}\right)$. Since $B$ is an arbitrary element of $\mathfrak{B}^{\sharp}$, it follows that $\mathfrak{B}$ centralizes every element of $И\left(\mathfrak{B} ;\{2, r\}^{\prime}\right)$.

Choose $\mathfrak{S} \in \operatorname{S}$ ol $(\mathfrak{B}), \mathfrak{B} \subseteq \mathfrak{S}$. Let $\mathfrak{S}_{0}=\boldsymbol{O}_{r},(\mathfrak{S})$. By the preceding argument, $C_{\widetilde{\Xi}_{0}}(\mathfrak{B})$ contains a $S_{2^{\prime}}$-subgroup of $\mathfrak{S}_{0}$. Since $C_{\mathfrak{5}}(\mathfrak{B}) \neq 1$, Lemma 6.2 implies that for some $C$ in $C(\mathfrak{B}), \widetilde{S}_{0} \cap \mathfrak{S C}^{C}$ contains a $S_{2^{-}}$ subgroup of $\mathfrak{S}_{0}$. Since $\mathfrak{S C}^{C} \subseteq \mathfrak{M}(\mathfrak{S}, \mathfrak{R})^{C}=\mathfrak{M}(\mathfrak{S}, \mathfrak{R})$, it follows that $\mathfrak{S}_{0} \subseteq \mathfrak{M}(\mathfrak{S}, \mathfrak{R})$.

If $\mathfrak{R}^{\prime}=1$, then $\mathfrak{S}_{0} \mathfrak{B} \triangleleft \mathfrak{S}$. Since $C_{\mathfrak{5}}(\mathfrak{B}) \neq 1$, it follows that $\mathfrak{M}(\mathfrak{S}, \mathfrak{R})$ covers $N(\mathfrak{B}) / C(\mathfrak{B})$. Since $\boldsymbol{C}(\mathfrak{B}) \subseteq \mathfrak{M}(\mathfrak{S}, \mathfrak{R})$, it follows that $N(\mathfrak{B}) \subseteq \mathfrak{M}(\mathfrak{S}, \mathfrak{R})$. Since $\mathfrak{S}=\mathfrak{S}_{0} \cdot N_{\widetilde{S}}(\mathfrak{B})$, we get $\mathfrak{S} \subseteq \mathfrak{M}(\mathfrak{S}, \mathfrak{R})$, and we are done.

Suppose $\mathfrak{R}^{\prime} \neq 1$. Let $\mathfrak{X}$ be a $S_{r}$-subgroup of $\subseteq \cap \mathfrak{M}(\mathfrak{S}, \mathfrak{R})$ which contains $\mathfrak{B}$ and let $\mathfrak{Y}$ be a $S_{r}$-subgroup of $\mathfrak{S}$ which contains $\mathfrak{X}$. Suppose $\mathfrak{X} \subset \mathfrak{Y}$. Let $\mathfrak{X}_{0}$ be an elementary subgroup of $\mathfrak{X}$ of order $r^{2}$ with $\mathfrak{X} \subset \boldsymbol{N}_{\mathfrak{9}}\left(\mathfrak{X}_{0}\right)$ 。 By Lemma 14.14 applied to $\mathfrak{X}_{0}$, we get

$$
C\left(\mathfrak{X}_{0}\right) \subseteq \mathfrak{M}\left(\mathfrak{S}_{\mathcal{C}}, \mathfrak{R}\right) \text {. }
$$

Since at least $r$ subgroups of $\mathfrak{X}_{0}$ of order $r$ have non trivial fixed points on $\mathfrak{F}$, Lemma 6.2 implies that $N(\mathfrak{S})$ covers $N\left(\mathfrak{X}_{0}\right) / C\left(\mathfrak{X}_{0}\right)$. Since $N(\mathfrak{S}) \subseteq \mathfrak{M}(\mathfrak{S}, \mathfrak{R})$, it follows that $N\left(\mathfrak{X}_{0}\right) \subseteq \mathfrak{M}(\mathfrak{S}, \mathfrak{R})$. Hence, $\mathfrak{X}=\mathfrak{Y}$.

Let $\mathfrak{X}_{1}=\mathfrak{X} \cap \boldsymbol{O}_{r^{\prime}, r}(\mathfrak{X})$. If $\mathfrak{X}_{1}$ is not of symplectic type, then $\mathfrak{X}_{1}$ contains a non cyclic characteristic elementary subgroup $\mathfrak{X}_{2}$. As above, we get $N\left(\mathfrak{X}_{2}\right) \subseteq \mathfrak{M}(\mathfrak{S}, \mathfrak{R})$. We may therefore assume that $\mathfrak{X}_{1}$ is of symplectic type. Since $\mathfrak{X}_{1}^{\prime} \subset \mathfrak{B}$, it follows that $C_{\mathfrak{5}}\left(\mathfrak{X}_{1}^{\prime}\right) \neq 1$. By Lemma 6.2 , it follows that $N\left(\mathfrak{F}_{\mathcal{C}}\right)$ covers $N\left(\mathfrak{X}_{1}\right) / C\left(\mathfrak{X}_{1}\right)$. Since $N(\mathfrak{S}) \subseteq \mathfrak{M}(\mathfrak{S}, \mathfrak{R})$, it follows that $\subseteq \subseteq \mathfrak{M}(\mathfrak{S}, \mathfrak{R})$. The proof is complete.

Lemma 14.22. Suppose $\mathfrak{B}$ is an elementary subgroup of $\mathfrak{R}$ of order $r^{2}, B \in \mathfrak{B}^{*}$ and $\boldsymbol{C}_{\mathfrak{5}}(B)$ contains an elementary subgroup which is normalized by $\mathfrak{B}$ but is not centralized by $\mathfrak{B}$. Then $N(\langle B\rangle) \subseteq \mathfrak{M}(\mathfrak{S E}, \mathfrak{R})$.

Proof. Set $\mathfrak{B}_{0}=\langle B\rangle, \mathfrak{N}=N\left(\mathfrak{B}_{0}\right), \quad \mathfrak{N}_{0}=\boldsymbol{O}_{r^{\prime}}(\mathfrak{R})$. Let $\tilde{\mathfrak{E}}$ be an elementary subgroup of $C_{\mathfrak{5}}(B)$ which admits $\mathfrak{B}$ and is not centralized by $\mathfrak{B}$. Set $\widetilde{F}=[\tilde{E}, \mathfrak{B}], \mathfrak{B}=\mathfrak{B}_{0} \times \mathfrak{B}_{1}$. Then $\mathfrak{B}_{1} \mathfrak{F}$ is a Frobenius group. 
Choose $q \in \pi\left(\mathfrak{N}_{0}\right), q \neq 2$, and let $\mathfrak{R}_{1}$ be a $S_{2, q}$-subgroup of $\mathfrak{R}_{0}$ which contains $\mathfrak{F}$ and admits $\mathfrak{B}$. Let $\left\{\mathfrak{R}_{2}, \mathfrak{R}_{q}\right\}$ be a Sylow system of $\mathfrak{R}_{1}$ which admits $\mathfrak{B}$ with $\mathfrak{F} \subseteq \mathfrak{N}_{2}$. Suppose by way of contradiction that $\left[\mathfrak{N}_{q}, \mathfrak{B}\right] \neq 1$. By Lemma $14.13, \boldsymbol{O}_{2}\left(\mathfrak{R}_{1} \mathfrak{B}\right)=1$, so $\mathfrak{F}$ is represented faithfully on $\boldsymbol{O}_{q}\left(\mathfrak{R}_{1} \mathfrak{B}\right)$. Let $\mathfrak{F}=\boldsymbol{C}(\mathfrak{B}) \cap \boldsymbol{O}_{q}\left(\mathfrak{N}_{1} \mathfrak{B}\right)$. Since $\mathfrak{B}_{0}$ centralizes $\mathfrak{R}_{0}$, and since $\mathfrak{B}_{1} \mathfrak{F}$ is a Frobenius group, it follows that $\langle\mathscr{F}$, $\mathfrak{F}\rangle$ contains an elementary subgroup of order $q^{3}$. On the other hand, $\langle\mathfrak{F}, \mathfrak{F}\rangle \subseteq \mathfrak{S} \mathfrak{D}$, where $\mathfrak{D}=\boldsymbol{O}_{r^{\prime}}(\boldsymbol{C}(\mathfrak{B}))$, by Lemma 14.15 . Since $\boldsymbol{O}_{2}(\mathfrak{S} \mathfrak{D}) \neq 1$, we get $e \geqslant 3$. This contradiction shows that $\mathfrak{B}$ centralizes a $S_{2}$-subgroup of $\mathfrak{R}_{0}$. $\mathfrak{N}_{0}$. By Lemma 6.2, there is $C$ in $C(\mathfrak{B})$ such that $\mathfrak{R}_{0} \cap \mathfrak{S}^{C}$ contains a $S_{2}$-subgroup of $\mathfrak{R}_{0}$. Thus, $\mathfrak{R}_{0} \subseteq \mathfrak{M}\left(\mathfrak{S}_{\mathcal{L}}, \mathfrak{R}\right)$.

Let $\mathfrak{Y}$ be a $S_{r}$-subgroup of $\mathfrak{R}$ which contains $\mathfrak{B}$. By Lemma 6.2 applied to $\mathfrak{Y} \cap \mathfrak{M}(\mathfrak{S}, \mathfrak{R})$, we get that $\mathfrak{Y} \subseteq \mathfrak{M}(\mathfrak{K}, \mathfrak{R})$. Let

$$
\mathfrak{Y}_{0}=\mathfrak{Y} \cap \boldsymbol{O}_{r^{\prime}, r}(\mathfrak{R}) \text {. }
$$

By Lemma 6.2 applied to $\mathfrak{N}_{0}$, we get $N_{\mathfrak{R}}\left(\mathfrak{V}_{0}\right) \subseteq \mathfrak{M}(\mathfrak{\mathcal { E }}, \mathfrak{R})$. The proof is complete.

\section{Hypothesis $14.2 \quad \sigma_{2} \nsubseteq\{3\}$.}

Lemmas 14.23 through 14.26 are proved under Hypothesis 14.2. We retain our earlier notation. Hence, $r \geqslant 5$. In the following lemmas, $\Re$ is a $S_{r}$-subgroup of $\mathscr{B}, \mathfrak{F}$ is a maximal element of $U(\Re ; 2)$, $\mathfrak{M}=\mathfrak{M}(\mathfrak{S}, \mathfrak{R}), \mathfrak{I}$ is a $\mathfrak{S}_{2}$-subgroup of $\mathfrak{M}$ which is permutable with $\mathfrak{R}$, $\mathfrak{S}_{0}=\boldsymbol{O}_{2}(\mathfrak{M})$, and $\mathfrak{B}=\Omega_{1}\left(\boldsymbol{R}_{2}(\mathfrak{M})\right)$, $\mathfrak{E}=\boldsymbol{C}_{\mathfrak{M}}(\mathfrak{B})$. Since $\mathfrak{B} \triangleleft \mathfrak{M} \in \mathscr{C} S(\mathbb{S})$, it follows that $\mathbb{C}=\boldsymbol{C}(\mathfrak{B})$.

As in $\S 13$, let $\mathscr{J}=\mathscr{I}(\mathfrak{M})$ be the set of all involutions $I$ of $\mathfrak{M}$ such that $C_{\mathfrak{M}}(I) \in \mathscr{M}^{*}(\mathbb{S})$, and let $\hat{\mathcal{J}}(\mathfrak{M})$ be the set of all involutions $I$ of $\mathfrak{M}$ such that

(a) $\boldsymbol{r} \| \boldsymbol{C}_{\mathfrak{n}}(I) \mid$,

(b) the normal closure of $I$ in $\mathfrak{M}$ is abelian. All of this notation will be used without further comment.

LEMMA 14.23. $\quad \mathscr{F}_{0} \Omega_{1}(\Re) \triangleleft \mathfrak{M}$ and if $\mathfrak{R} \subseteq \mathfrak{M}^{\prime}$, then $\mathfrak{F}_{0} \Re \triangleleft \mathfrak{M}$.

Proof. Let $\mathfrak{Z}=\boldsymbol{O}_{r^{\prime}}(\mathfrak{M}) \quad$ By Lemma 14.2, $\mathfrak{R} \triangleleft \mathfrak{M}$. Also, $\mathfrak{Z}=$ $\mathfrak{S}\left(\mathcal{D}\right.$, where $\mathfrak{D}$ is a $S_{2}$-subgroup of $\mathbb{Z}$ which admits $\Re$. By Lemma 14.16 and $14.18, \Omega_{1}(\Re)$ centralizes $\mathfrak{D}$. Let $\mathfrak{F}=\boldsymbol{F}\left(\mathbb{R} \bmod \mathfrak{S}_{0}\right)$, so that $\mathfrak{F}=\mathfrak{S}_{0}(\mathfrak{F} \cap \mathfrak{D})$. Thus, $\Omega_{1}(\Re)$ centralizes $\mathfrak{F} / \mathfrak{S}_{0}$. Since

$$
\boldsymbol{C}_{L}\left(\mathfrak{F} / \mathfrak{S}_{0}\right)=\boldsymbol{Z}\left(\mathfrak{F} \bmod \mathfrak{S}_{0}\right),
$$

it follows that $\Omega_{1}(\Re)$ stabilizes the chain $\mathbb{R} / \mathscr{S}_{0} \supseteqq \mathfrak{F} / \mathfrak{S}_{0} \supseteqq 1$. Since $\mathbb{Z}$ is a $r^{\prime}$-group, $\Omega_{1}(\Re)$ centralizes $\mathfrak{R} / \mathfrak{S}_{0}$. Hence, 


$$
\Omega_{1}(\Re) \mathfrak{R} / \mathfrak{S}_{0}=\mathfrak{L} / \mathfrak{S}_{0} \times \Omega_{1}(\Re) \mathfrak{S}_{0} / \mathfrak{S}_{0},
$$

so $\Omega_{1}(\Re) \mathfrak{S}_{0}$ char $\Omega_{1}(\Re)$ char $\mathbb{R} \triangleleft \mathfrak{M}$. This establishes the first assertion. If $[\Re, \mathfrak{D}]=1$, the second assertion follows by the same argument. Suppose $[\mathfrak{D}, \Re] \neq 1$. Let $\mathfrak{Q}$ be a $S_{q}$-subgroup of $\mathfrak{D}$ which admits $\Re$ and satisfies $[\mathfrak{\Omega}, \Re] \neq 1$. By Lemma 14.3 , we have $q>r$. By definition of $r, \mathfrak{Q}$ is cyclic and is a $S_{q}$-subgroup of $\mathfrak{M}$. Thus, $\mathfrak{Q}$ is incident with $\boldsymbol{O}_{q^{\prime}, q}(\mathfrak{M}) / \boldsymbol{O}_{q^{\prime}}(\mathfrak{M})$ and is not cetralized by $\Re$. Hence, $\Re \nsubseteq \mathfrak{M}^{\prime}$, since $\mathfrak{M}^{\prime}$ centralizes $\boldsymbol{O}_{q^{\prime}, q}(\mathfrak{M}) / \boldsymbol{O}_{q^{\prime}}(\mathfrak{M})$, and $\mathfrak{R}$ does not. The proof is complete.

LEMMA 14.24. If $\tilde{\mathfrak{R}}$ is any subgroup of $\mathfrak{R}$ which is permutable with $\mathfrak{T}$, then $\tilde{\mathfrak{R}}=(\tilde{\Re} \cap \mathfrak{E})(\tilde{\Re} \cap N(J(\mathfrak{T})))$. Furthermore, every characteristic subgroup of $\mathfrak{R}$ is permutable with $\mathfrak{I}$.

Proof. By Lemma 14.2, $l_{r}(\mathfrak{M})=1$, and so the final statement of the lemma holds.

Since $r \geqslant 5$, Theorem 1 of [43] applied to $\mathfrak{T} \tilde{\Re}$ implies that $\tilde{\mathfrak{R}}=$ $(\tilde{\Re} \cap \boldsymbol{C}(\boldsymbol{Z}(\mathfrak{T})))(\tilde{\Re} \cap \boldsymbol{N}(\boldsymbol{J}(\mathfrak{T})))$. Thus, it suffices to show that

$$
\tilde{\mathfrak{R}} \cap \boldsymbol{C}(\boldsymbol{Z}(\mathfrak{T})) \subseteq \mathfrak{F}
$$

Let $\mathfrak{Z}=\mathfrak{T} \mathfrak{R}_{0}$, where $\mathfrak{R}_{0}=\tilde{\mathfrak{R}} \cap \boldsymbol{C}(\boldsymbol{Z}(\mathfrak{T}))$. Since $\mathfrak{T} \tilde{\mathfrak{R}}$ is a group by hypothesis, we get $\mathfrak{Q}=\boldsymbol{C}_{\mathfrak{\Sigma} \tilde{\mathfrak{n}}}(\boldsymbol{Z}(\mathfrak{T}))$.

Case 1. $\Re \cong \mathfrak{M}^{\prime}$.

By Lemma 14.24, we heve $\mathfrak{S}_{0} \Re \triangleleft \mathfrak{M}$. Hence, $\mathfrak{S}_{0} \Re_{0} \triangleleft \mathfrak{R}$. Since $\mathfrak{B} \subseteq \boldsymbol{R}_{2}(\mathfrak{M})$, it follows that $\mathfrak{S}_{0} \subseteq \mathfrak{C}$. Thus, we may view $\mathfrak{B}$ as a $\mathfrak{L} / \mathfrak{S}_{0}$-module. Hence, $\left[\mathfrak{B}, \mathfrak{R}_{0}\right] \triangleleft \mathfrak{R}$. If $\left[\mathfrak{B}, \mathfrak{R}_{0}\right] \neq 1$, then

$$
\left[\mathfrak{B}, \Re_{0}\right] \cap Z(\mathfrak{I}) \neq 1 \text {, }
$$

against $\boldsymbol{Z}(\mathfrak{I}) \subseteq C\left(\Re_{0}\right)$ and $\left[\mathfrak{B}, \Re_{0}\right] \cap \boldsymbol{C}\left(\Re_{0}\right)=1$.

Case 2. $\mathfrak{R} \nsubseteq \mathfrak{M}^{\prime}$.

By Lemmas 14.10 and 14.19 , we get $\Re^{\prime}=1$. Let $\mathfrak{B}=\Omega_{1}(\Re)$. Since $\Re$ is an abelian Sylow subgroup of $\left(\mathfrak{S}, \boldsymbol{A}_{\circledast}(\mathfrak{B}) \cong \boldsymbol{A}_{\Theta}(\mathfrak{R})\right.$. Hence, $\mathfrak{B}$ contains elements $B_{1}, B_{1}$ which are $(5-$-conjugate but are not $\mathfrak{M}$ conjugate. By Lemma 14.19, we conclude that $C_{\mathfrak{s}}(\mathfrak{B})=1$. By Lemma $14.23, \mathfrak{S}_{0} \mathfrak{B} \triangleleft \mathfrak{M}$, and so $\mathfrak{B}$ centralizes $\mathfrak{S}_{\mathfrak{C}} / \mathscr{S}_{0}$. Since $C_{\mathfrak{F}}(\mathfrak{B})=1$, we get $\mathfrak{S}=\mathfrak{S}_{0}$.

Since $l_{r}(\mathfrak{M})=1$, we get $\mathfrak{F}_{0} \Re \triangleleft \mathfrak{T} \Re$. We conclude that $\mathfrak{S}_{0} \Re \triangleleft \mathfrak{R}$, so the argument of Case 1 applies. The proof is complete. 
The next lemma is the heart of the matter, and is difficult.

LEMMA 14.25. $\hat{\mathcal{J}}(\mathfrak{M}) \subseteq \mathscr{J}(\mathfrak{M})$.

Proof. Let $\mathscr{S}$ be the set of all solvable subgroups $\mathbb{S}$ of $\mathbb{B}$ such that

(a) $\subseteq \nsubseteq \mathfrak{M}$.

(b) There is $I$ in $\hat{\mathscr{J}}$ such that $\subseteq$ contains a $S_{2, r}$-subgroup of $\boldsymbol{C}_{\mathfrak{m}}(I)$. Suppose $\mathscr{S}=\varnothing$. Then $\boldsymbol{C}_{\mathfrak{m}}(I) \in \mathscr{M}^{*}(\mathbb{S})$ for all $I$ in $\hat{\mathscr{F}}$, and so the lemma follows by definition of $\mathscr{F}$. We may assume by way of contradiction that $\mathscr{S} \neq \varnothing$.

Among all elements of $\mathscr{S}$, choose $\subseteq$ such that

(a) $|\mathfrak{S} \cap \mathfrak{M}|_{2} \geqslant\left|\mathfrak{S}_{0} \cap \mathfrak{M}\right|_{2}$ for all $\mathfrak{S}_{0}$ in $\mathscr{S}$,

(b) if $\mathfrak{S}_{0} \in \mathscr{S}$, and $|\mathfrak{S} \cap \mathfrak{M}|_{2}=\left|\mathfrak{S}_{0} \cap \mathfrak{M}\right|_{2}$, then $|\mathfrak{S}|_{2} \geqslant\left|\mathfrak{S}_{0}\right|_{2}$,

(c) if $\mathfrak{S}_{0} \in \mathscr{S}$ and $|\mathfrak{S} \cap \mathfrak{M}|_{2}=\left|\mathfrak{S}_{0} \cap \mathfrak{M}\right|_{2}$ and $|\mathfrak{S}|_{2}=\left|\mathfrak{S}_{0}\right|_{2}$, then $|\pi(\Im)| \leqq\left|\pi\left(\Im_{0}\right)\right|$.

Choose $I$ in $\hat{\mathcal{J}}$ and a $S_{2, r}$-subgroup $\Re$ of $C_{\mathfrak{m}}(I)$ such that $\Re \subseteq \subseteq$.

Case 1. $S_{r}$-subgroups of $\Omega$ are non cyclic.

Let $\mathfrak{B}$ be an elementary subgroup of $\Re$ of order $r^{2}$. Since $\mathfrak{B} \subseteq \subseteq$ and $\mathfrak{S} \nsubseteq \mathfrak{M}$, it follows that $\mathfrak{B} \notin \mathfrak{M}^{*}(\mathbb{S})$. By Lemma 14.21, $\boldsymbol{C}_{\mathfrak{F}}(\mathfrak{B})$ contains no four-group. Thus, $I$ is the only involution of $C_{\mathfrak{F}}(\mathfrak{B})$. (Note that by definition of $\hat{\mathcal{F}}$, we have $\langle I\rangle^{M} \cong \mathfrak{S}_{0}=\boldsymbol{O}_{2}(\mathfrak{M}) \subseteq \mathfrak{H}_{2}$.)

Let $\mathfrak{M}_{0}$ be a $S_{2, r}$-subgroup of $\mathfrak{M} \cap \mathfrak{S}$ which contains $\Re$ and let $\mathfrak{S}_{0}$ be a $S_{2, r}$-subgroup of $\subseteq$ which contains $\mathfrak{M}_{0}$. Let $\mathfrak{N}_{0}=\boldsymbol{O}_{2}\left(\mathfrak{M}_{0}\right)$. By Lemma 14.7, $\mathfrak{N}_{0}$ is the subgroup of $\mathfrak{M}_{0}$ generated by all the elements

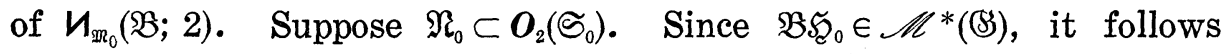
that $\mathfrak{S}_{0} \nsubseteq \subseteq$. Hence, $\mathfrak{N}_{0} \subset \mathfrak{N}_{0} \mathfrak{S}_{0}$. Hence, $\left|\boldsymbol{N}_{\mathfrak{M}}\left(\mathfrak{N}_{0}\right)\right|_{2}>\left|\mathfrak{M}_{0}\right|_{2}$. On the other hand, since $\mathfrak{R}_{0} \subset \boldsymbol{O}_{2}\left(\mathfrak{S}_{0}\right)$, it follows that $N\left(\mathfrak{N}_{0}\right) \nsubseteq \mathfrak{M}$. Hence, $N\left(\mathfrak{R}_{0}\right) \in \mathscr{S}$ and $\left|N\left(\mathfrak{M}_{0}\right) \cap \mathfrak{M}\right|_{2}>|\mathfrak{S} \cap \mathfrak{M}|_{2}$, against our choice of $\mathfrak{S}$. We conclude that $\mathfrak{R}_{0}=\boldsymbol{O}_{2}\left(\mathfrak{S}_{0}\right)$. Here we are again using Lemma 14.7 to conclude that $\mathfrak{N}_{0} \subseteq \boldsymbol{O}_{2}\left(\mathfrak{S}_{0}\right)$.

Since $\mathfrak{B} \mathfrak{S}_{0} \in \mathscr{C}^{*}(\mathbb{S})$, we get $\mathfrak{S}_{0} \nsubseteq \mathfrak{S}$. Hence,

$$
\left|\boldsymbol{N}_{\mathfrak{M}}\left(\mathfrak{R}_{0}\right)\right|_{2}>|\subseteq \cap \mathfrak{M}|_{2},
$$

and so $N\left(\mathfrak{R}_{0}\right) \subseteq \mathfrak{M}$. In particular, $\mathfrak{S}_{0}=\mathfrak{M}_{0}$, and so $\mathfrak{S}$ is not a $2, r$ group. The minimality of $|\pi(\mathfrak{S})|$ implies that $\subseteq=\mathfrak{S}_{0} \Omega$, where $\mathfrak{Q}$ is a $q$-group for some prime $q \neq 2, r$.

Let $\left\{\mathfrak{S}_{2}, \mathfrak{S}_{r}\right\}$ be a Sylow system of $\mathfrak{S}_{0}$ which is permutable with

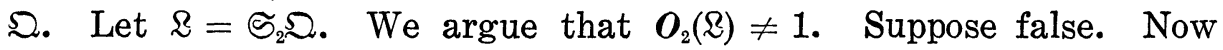
$\boldsymbol{C}_{\mathfrak{5}_{0}}(I) \subseteq \widetilde{S}_{2}$ and $I$ is the only involution of $\boldsymbol{C}_{\mathfrak{5}}(B)$. On the other 
hand, by definition of $\hat{\mathscr{F}}$, the normal closure of $I$ in $\mathfrak{M}$ is abelian, so $C_{\$_{0}}(I)$ contains an elementary subgroup $(\xi$ of order 16 . Thus, $₹$ is represented faithfully on $\boldsymbol{O}_{q}(\mathfrak{Q})$, so by Lemma 5.34, (अ contains an element $E$ such that $\boldsymbol{O}_{q}(\mathbb{R}) \cap \boldsymbol{C}(\mathfrak{S})$ contains an elementary subgroup of order $q^{3}$. Since $\langle E\rangle \in \boldsymbol{U}\left(\boldsymbol{O}_{q}(\mathbb{R}) \cap \boldsymbol{C}(E)\right.$; 2), it follows that

$$
q \notin \sigma_{0} \cup \sigma_{1} \cup \sigma_{2} \text {. }
$$

This contradiction implies that $O_{2}(\Omega) \neq 1$.

Since $\boldsymbol{C}_{\mathfrak{w}}(\mathfrak{B}) \neq 1$, Lemma 14.7 implies that

$$
N(\mathfrak{B})=C(\mathfrak{B}) \cdot(N(\mathfrak{B}) \cap N(\mathfrak{F})) .
$$

By Lemmas 14.17, 14.18, and 14.19, we get $N(\mathfrak{B}) \subseteq \mathfrak{M}$. In particular, $\mathfrak{B}$ does not centralize $\mathfrak{\Omega}$. Since $\boldsymbol{O}_{2}(\mathfrak{R}) \neq 1$, we get $q \in \sigma_{1} \cup \sigma_{2}$. Suppose $q<r$. In this case, since $\mathscr{S} c n_{3}(\mathfrak{\Omega})=\varnothing$ and $\mathscr{S} c n_{3}\left(\mathbb{S}_{r}\right)=\varnothing$, it follows that $\mathfrak{S}_{r} \triangleleft \mathfrak{S}_{r} \mathfrak{O}$, by Lemma 0.8.5. Thus, $\mathfrak{S}_{r}$ is non abelian, since $\mathfrak{B} \pitchfork \mathfrak{S}_{r} \mathfrak{S}$. If $\mathfrak{S}_{r}$ is of symplectic type, then $\mathfrak{S}_{r}^{\prime} \subseteq \mathfrak{B}$ and since $\boldsymbol{C}_{\mathfrak{5}}\left(\mathfrak{S}_{r}^{\prime}\right) \neq 1$, Lemma 14.7 implies that $N\left(\mathfrak{S}_{r}\right) \subseteq \mathfrak{M}$. This is not the case, so $\mathfrak{S}_{r}$ contains a non cyclic characteristic elementary abelian subgroup $\mathfrak{B}_{0}$. Since $I$ is the only involution of $C_{\mathfrak{g}}(\mathfrak{B})$ and since $\mathfrak{B}_{0}$ normalizes $\mathfrak{B}$, it follows that $\mathfrak{B}_{0}$ centralizes $I$. Thus, we may assume that $\mathfrak{B}_{0}=\mathfrak{B}$. This contradiction forces $q>r$.

By maximality of $r$, we get $q \in \sigma_{1}$, and so $\cong$ is cyclic. Since $q>r$, we get $\mathfrak{\mathfrak { D }}$. Let $\mathfrak{S}^{0}=\mathfrak{N}_{0} \mathfrak{\mathfrak { Q }}=\boldsymbol{O}_{r},(\mathfrak{S})$. Since $\mathfrak{R}_{0}$ contains a four-group, we get $\boldsymbol{O}_{2}\left(\Im^{0}\right) \neq 1$. Hence, $\boldsymbol{O}_{2}(\mathfrak{S}) \neq 1$. Since $\Omega \nsubseteq \mathfrak{M}$, we also have $[\mathfrak{\Omega}, \mathfrak{B}] \neq 1$. This violates Lemma 14.13.

Case 2. $S_{r}$-subgroups of $\Re$ are cyclic.

By definition of $\hat{\mathscr{I}}$, a $S_{r}$-subgroup $\Re_{r}$ of $\Re$ is a non trivial cyclic group. Let $\mathfrak{A}=\Omega_{1}\left(\Re_{r}\right)$, and let $\mathfrak{B}$ be an elementary subgroup of $\mathfrak{M}$ of order $r^{2}$ which contains $\mathfrak{U}$. Since $\mathfrak{B}$ does not centralize $I$ and since $\langle I\rangle^{M}$ is abelian, Lemma 14.22 implies that $N(\mathfrak{l}) \subseteq \mathfrak{M}$.

Case 2a. $S_{r}$-subgroups of $\mathfrak{M} \cap \subseteq$ are non cyclic.

Again, let $\mathfrak{M}_{0}$ be a $S_{2, r}$-subgroup of $\mathfrak{M} \cap \subseteq$ which contains $\Re$ and let $\mathfrak{S}_{0}$ be a $S_{2, r}$-subgroup of $\subseteq$ which contains $\mathfrak{M}_{0}$. Let $\mathfrak{N}_{0}=$ $\boldsymbol{O}_{2}\left(\mathfrak{M}_{0}\right)$. By Lemma $14.20, \mathfrak{S}_{0} \nsubseteq \mathfrak{R}_{0}$, so $\left|\boldsymbol{N}_{\mathfrak{m}}\left(\mathfrak{R}_{0}\right)\right|_{2}>\left|\mathfrak{M}_{0}\right|_{2}$. This implies that $N\left(\mathfrak{N}_{0}\right) \subseteq \mathfrak{M}$ and that $\mathfrak{M}_{0}=\mathfrak{S}_{0}$. By minimality of $|\pi(\mathfrak{S})|$, we get $\mathfrak{S}=\mathfrak{S}_{0} \mathfrak{Q}$, where $\mathfrak{D}$ is a $q$-group for some odd prime $q \neq 2, r$. As above, we get a contradiction to Lemma 14.13.

Case 2b. $S_{r}$-subgroups of $\mathfrak{M} \cap \subseteq$ are cyclic. 
Again, let $\mathfrak{M}_{0}$ be a $S_{2, r}$-subgroup of $\mathfrak{M} \cap \subseteq$ which contains $\Re$ and let $\mathfrak{S}_{0}$ be a $S_{2, r}$-subgroup of $\mathfrak{S}$ which contains $\mathfrak{M}_{0}$. Let $\left\{\mathfrak{M}_{2}, \mathfrak{M}_{r}\right\}$, $\left\{\mathfrak{S}_{2}, \mathfrak{S}_{r}\right\}$ be Sylow systems of $\mathfrak{M}_{0}, \mathfrak{S}_{0}$ respectively with $\mathfrak{R}_{r} \subseteq \mathfrak{M}_{r} \subseteq \mathfrak{S}_{r}$. Since $N(\mathfrak{U}) \subseteq \mathfrak{M}$ and $\mathfrak{M}_{r}$ is cyclic, we get $\mathfrak{M}_{r}=\mathfrak{S}_{r}$.

Since $\mathfrak{M}_{r}=\widetilde{S}_{r}$, it follows that $\boldsymbol{O}_{2}\left(\mathfrak{M}_{0}\right) \subseteq \boldsymbol{O}_{2}\left(\widetilde{S}_{0}\right)$.

Case $2 b(\mathrm{i}) . \quad \boldsymbol{O}_{2}\left(\mathfrak{M}_{0}\right) \subset \boldsymbol{O}_{2}\left(\Im_{0}\right)$.

In this case, we get $N\left(\boldsymbol{O}_{2}\left(\mathfrak{M}_{0}\right)\right) \nsubseteq \mathfrak{M}$, so by maximality of $\mid \subseteq \cap \mathfrak{M}_{2}$, we get that $\mathfrak{M}_{2}$ is a $S_{2}$-subgroup of $N_{\mathfrak{N}}\left(\boldsymbol{O}_{2}\left(\mathfrak{M}_{0}\right)\right)$. In particular, $\mathfrak{S}_{0} \subseteq \boldsymbol{O}_{2}\left(\mathfrak{M}_{0}\right)$.

We assume without loss of generality that $\mathfrak{M}_{2} \subseteq \mathfrak{I}, \mathfrak{M}_{r} \subseteq \Re$. Since $\mathfrak{M}_{2}$ is a $\mathfrak{S}_{2}$-subgroup of $\boldsymbol{N}_{\mathfrak{n}}\left(\boldsymbol{O}_{2}\left(\mathfrak{M}_{0}\right)\right)$, it follows that $\boldsymbol{O}_{2}\left(\mathfrak{M}_{0}\right)$ is a maximal element of $\boldsymbol{V}_{\mathfrak{R}}(\mathfrak{A} ; 2)$, and so $\boldsymbol{O}_{2}\left(\mathfrak{M}_{0}\right) \supseteqq \mathfrak{K}_{\text {. }}$ Hence, $\boldsymbol{O}_{2}\left(\mathfrak{M}_{0}\right)$ is permutable with $\mathfrak{R}$ and with $\Omega_{1}(\Re)$. By Theorem 1 of [43] applied to $\boldsymbol{O}_{2}\left(\mathfrak{M}_{0}\right) \Omega_{1}(\Re)$, it follows that $\boldsymbol{O}_{2}\left(\mathfrak{M}_{0}\right)$ contains a characteristic subgroup $\mathfrak{X} \neq 1$ such that $N_{\mathfrak{R}}(X)$ is non cyclic. Since $\boldsymbol{O}_{2}\left(\mathfrak{M}_{0}\right) \subset \boldsymbol{O}_{2}\left(\Im_{0}\right)$, it follows that $N(\mathfrak{X}) \nsubseteq \mathfrak{M}$. This is not the case by Lemma 14.20 .

Case $2 \mathrm{~b}(\mathrm{ii}) . \quad \boldsymbol{O}_{2}\left(\mathfrak{M}_{0}\right)=\boldsymbol{O}_{2}\left(\Im_{0}\right)$.

Since $\mathfrak{S}_{r}=\mathfrak{M}_{r}$ is cyclic, $\mathfrak{S}_{0}=\boldsymbol{O}_{2}\left(\mathfrak{S}_{0}\right) \boldsymbol{N}_{\mathfrak{\Xi}_{0}}(\mathfrak{Z})$, and so $\mathfrak{S}_{0} \subseteq \mathfrak{M}$. By minimality of $|\pi(S)|$, we get $\subseteq=S_{0} \mathfrak{N}$, where $\mathfrak{Q}$ is a $q$-group for some prime $q \neq 2, r$, and where $\mathfrak{Q}$ is permutable with $\mathfrak{S}_{2}$ and with $\mathfrak{S}_{r}$.

We argue that $\boldsymbol{O}_{2}\left(\mathfrak{S}_{2} \mathfrak{\Omega}\right) \neq 1$. Suppose false. Since $\Re \subseteq \mathfrak{S}$, it follows that $\widetilde{S}_{2}$ contains an elementary subgroup $(5$ of order 8 . Since (5) is represented faithfully on $\boldsymbol{O}_{q}\left(\mathfrak{S}_{2} \mathfrak{Q}\right)$, it follows that there is $E$ in $\mathbb{F}^{\#}$ such that $\boldsymbol{O}_{q}\left(\mathfrak{S}_{2} \mathfrak{\Omega}\right) \cap \boldsymbol{C}(E)$ is non cyclic. Hence, $q \in \sigma_{2}$. By definition of $r$, we get $r>q$, and by Lemma 14.8, we get $q \in \pi_{2}$. By Lemma 0.8.5, we get $\mathfrak{M}_{r} \triangleleft \mathfrak{M}_{r} \mathfrak{\Omega}$, and so $\mathfrak{Q} \subseteq \mathfrak{M}$. This is not the case, so $\boldsymbol{O}_{2}\left(\mathfrak{S}_{2} \mathfrak{\Im}\right) \neq 1$.

Since $\boldsymbol{O}_{2}\left(\mathfrak{S}_{2} \mathfrak{Q}\right) \neq 1$, we have $q \in \sigma_{1} \cup \sigma_{2}$. If $q \in \sigma_{2}$, then by Lemma 14.8, $q \in \pi_{2}$, and by definition of $r$, we get $q<r$. By Lemma 0.8.5, we get $\mathfrak{M}_{r} \triangleleft \mathfrak{M}_{r} \mathfrak{}$. This implies that $\mathfrak{M}$. We conclude that $q \in \sigma_{1}$, so that $\mathfrak{Q}$ is cyclic.

Since $\cong \nsubseteq \mathfrak{M}$ and $N(\mathfrak{N}) \subseteq \mathfrak{M}$, it follows that $\mathfrak{M}_{r} \mathfrak{Q}$ is a Frobenius group with kernel $\mathfrak{\Omega}$. This implies that $\mathfrak{M} \cap \mathfrak{M}=1$, since $\mathfrak{P}$ centralizes a $S_{2^{\prime}}$-subgroup of $\boldsymbol{O}_{r^{\prime}}(\mathfrak{M})$.

Since $\mathfrak{A}$ does not centralize $\mathfrak{Q}, \mathfrak{A}$ does not centralize $\boldsymbol{O}_{q^{\prime}, q}(\mathfrak{S}) / \boldsymbol{O}_{q^{\prime}}(\mathfrak{S}) \cong$ $\mathfrak{\Omega}$, and so $\mathfrak{N} \nsubseteq \mathfrak{S}^{\prime}$. Since $\mathfrak{U}=\Omega_{1}\left(\mathfrak{M}_{r}\right)$, it follows that $\mathfrak{M}_{2} \mathfrak{Q} \triangleleft \mathfrak{S}$. Since $q \equiv 1(\bmod r)$, it follows that $q>7$.

We next show that $\boldsymbol{O}_{q}\left(\mathfrak{M}_{2} \mathfrak{D}\right)=1$. Suppose false. Let $\mathfrak{D}=\boldsymbol{O}_{2}(\mathfrak{S})$, 
so that $\mathfrak{D} \neq 1$. Let $\mathfrak{S}^{*}=N(\mathfrak{D})$. Then $\Omega_{1}(\mathfrak{D}) \subseteq \mathfrak{S}^{*}$, and so $\mathfrak{S}^{*} \in \mathscr{S}$. This implies that $\mathfrak{M}_{2}$ is a $S_{2}$-subgroup of $\mathfrak{S}^{*}$, by the extremal nature

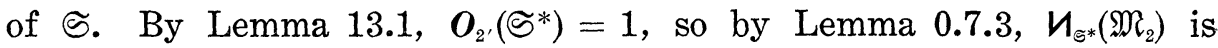
trivial, against $\boldsymbol{O}_{q}\left(\mathfrak{M}_{2} \mathfrak{O}\right) \in \boldsymbol{h}_{\mathfrak{S}^{*}}\left(\mathfrak{M}_{2}\right)$. This contradiction shows that $\boldsymbol{O}_{q}\left(\mathfrak{M}_{2} \mathfrak{\Omega}\right)=1$.

Since $q \geqslant 7$, it follows from Theorem 1 of [43] that $\mathfrak{M}_{2}$ contains a characteristic subgroup $\mathfrak{Y} \neq 1$ such that $\mathfrak{Y} \triangleleft \mathfrak{M}_{2} \Omega$. Hence, $\mathfrak{Y} \triangleleft \mathfrak{S}$, since $\mathfrak{M}_{2} \mathfrak{M}_{r}$ is 2-closed.

If $\mathfrak{M}_{2}$ is not a $S_{2}$-subgroup of $\mathbb{B}$, then $\mathfrak{M}_{2}$ is not a $S_{2}$-subgroup of $N(\mathfrak{Y})$. Since $\subseteq \subseteq N(\mathfrak{Y})$, we have $|\subseteq \cap \mathfrak{M}|_{2} \leqq|N(\mathfrak{Y}) \cap \mathfrak{M}|_{2}$, and since $|\mathfrak{S}|_{2}=|\mathfrak{M}|_{2}<|\boldsymbol{N}(\mathfrak{Y})|_{2}$, the extremal nature of $\subseteq$ is violated. We conclude that $\mathfrak{M}_{2}=\mathfrak{I}$ is a $S_{2}$-subgroup of $(\mathbb{S}$.

Let $\mathfrak{X}_{1}=\boldsymbol{Z}(\mathfrak{I}), \mathfrak{X}_{2}=\boldsymbol{J}(\mathfrak{T}), \mathfrak{X}_{3}=\boldsymbol{Z}\left(\boldsymbol{J}_{1}(\mathfrak{I})\right)$. Let

$$
\begin{aligned}
N & =\left\{i \mid 1 \leqq i \leqq 3, N_{\Re}\left(\mathfrak{X}_{i}\right) \text { is non cyclic }\right\}, \\
N^{\prime} & =\left\{i \mid 1 \leqq i \leqq 3, \mathfrak{X}_{i} \triangleleft \mathfrak{I} \mathfrak{\Omega}\right\} .
\end{aligned}
$$

Suppose $N \cap N^{\prime} \neq \varnothing$. Choose $i \in N \cap N^{\prime}$. By Lemma 14.20, we get $N_{\mathfrak{R}}\left(\mathfrak{X}_{i}\right) \in \mathscr{C}^{*}(\mathbb{S})$, and so $\subseteq \subseteq \mathfrak{M}$. This is not the case, so $N \cap N^{\prime}=$ $\varnothing$. Since $q \geqslant 7$, Lemma 5.53 implies that $\left|N^{\prime}\right| \geqslant 2$. Hence, $|N| \leqq 1$. Since $\mathfrak{A} \subseteq N_{\mathfrak{R}}\left(\mathfrak{X}_{i}\right)$ for $i=1,2,3$, Lemma 5.53 implies that $r=5$. Also, $N_{\Re}(\mathfrak{T})$ is cyclic.

Suppose $1 \in N$ and that $\mathfrak{A}$ centralizes $Z(\mathfrak{T})$. Then $1 \notin N^{\prime}$, so $\subseteq$ contains a minimal normal subgroup which is not centralized by $\mathfrak{\Omega}$. This implies that $\mathfrak{A}$ does not centralize $\boldsymbol{Z}(\mathfrak{T})$, by a well known property of Frobenius groups. We conclude that either $1 \notin N$ or $[Z(\mathfrak{Z}), \mathfrak{N}] \neq 1$.

Case $\alpha . \quad \mathfrak{2} \subseteq \Omega_{1}(\Re)^{\prime}$.

Let $\mathfrak{B}=\Omega_{1}(\mathfrak{R}), \mathfrak{R}=\mathfrak{T} \mathfrak{B}$, so that $\mathfrak{S} \mathfrak{B} \triangleleft \mathfrak{L}$. Since $\mathfrak{A}$ normalizes $\mathfrak{T}$, it follows that each element of $\mathfrak{I}$ induces an automorphism of $\mathfrak{S} \mathfrak{B} / \mathfrak{S} \mathfrak{Z}$ of determinant 1 (where we have identified $\mathfrak{S} \mathfrak{B} / \mathfrak{S} \mathfrak{A}$ with a 2-dimensional vector space over $F_{r}$ ). Thus, $\mathfrak{I} / \mathfrak{S}$ is isomorphic to a subgroup of $S L(2,5)$, whose $S_{2}$-subgroups are quaternion. If 2 does not centralize $\mathfrak{B}$, it follows by an easy omitted argument that $J_{1}(\mathfrak{I}) \subseteq \mathfrak{S}_{\mathfrak{E}}$, which gives $|N| \geqslant 2$. This is not the case, so $\mathfrak{A}$ centralizes $\mathfrak{B}$, so that $\mathfrak{A}$ centralizes $\boldsymbol{Z}(\mathfrak{I})$. Hence, $1 \notin N$. Since the unique involution of $\mathfrak{I} / \mathfrak{S}$ inverts $\mathfrak{S}_{\mathfrak{B}} / \mathfrak{S}_{\mathcal{2}} \mathfrak{A}$, it follows that $J_{1}(\mathfrak{T}) \subseteq \mathfrak{S}_{\mathfrak{E}}$, so that $|N| \geqslant 2$. This contradiction shows that this case does not arise.

Case $\beta . \quad \mathfrak{Q} \nsubseteq \mathfrak{B}^{\prime}$ and $\mathfrak{R}^{\prime} \neq 1$ (where $\mathfrak{B}=\Omega_{1}(\mathfrak{R})$ ).

By Lemmas 14.10 and 14.19, we have $\Re \subseteq \mathfrak{M}^{\prime}$. By Lemma 14.2, 
$\Re \subseteq N_{\mathfrak{R}}(\Re)^{\prime}$. By 0.3 .4 , one of the following holds:

(i) $\Re$ is the central product of a cyclic group and $\mathfrak{B}$.

(ii) $\Re=g p\langle A, B|[B, A]=C, \quad[C, A]=B^{s^{5 n-1}}, \quad C^{5}=[B, C]=$ $\left.A^{5}=B^{5^{n}}=1, n>1,(s, 5)=1\right\rangle$.

Here we also have used 0.3 .8 to conclude that $\Re$ is not metacyclic. If $\beta$ (i) holds, then $\mathfrak{R}^{\prime}=\mathfrak{B}^{\prime}$, and if $\beta$ (ii) holds, $\mathfrak{R}^{\prime}=\left\langle C, B^{5^{n-1}}\right\rangle$. In both cases, $\mathfrak{A}^{\mathfrak{R}}$ is non cyclic.

If $[\mathfrak{A}, \mathfrak{B}]=1$, then since $\mathfrak{B} \triangleleft \mathfrak{M}$, we get $\left[\mathfrak{U}^{\mathfrak{R}}, \mathfrak{B}\right]=1$, and so $1 \in N$, since $\boldsymbol{Z}(\mathfrak{I}) \subseteq \boldsymbol{R}_{2}(\mathfrak{M})$. This is false, since we have already shown that either $1 \notin N$ or $[\mathfrak{U}, \boldsymbol{Z}(\mathfrak{T})] \neq 1$. We conclude that $[\mathfrak{A}, \mathfrak{B}] \neq 1$.

Suppose $1 \in N$. Then $2,3 \notin N$. But the proof of Lemma 5.53 shows that $\Re=\left(\Re \cap N\left(\mathfrak{X}_{2}\right)\right)\left(\Re \cap N\left(\mathfrak{X}_{3}\right)\right)$, and so either $2 \in N$ or $3 \in N$. It follows that $1 \notin N$. By Lemma 5.53, we get $2 \in N$, and so $3 \notin N$.

Now $\mathfrak{A}$ normalizes $\mathfrak{T}$, and so $\mathfrak{A} \nsubseteq(\mathfrak{T} \mathfrak{B})^{\prime}$. But $\mathfrak{A} \subseteq \mathfrak{M}^{\prime}$, and so $\mathfrak{U} \subseteq \mathfrak{M}_{1}^{\prime}$, where $\mathfrak{M}_{1}=N_{\mathfrak{M}}(\mathfrak{R})$. This implies that $\boldsymbol{A}_{\mathfrak{M}}(\mathfrak{B})$ is not a $\{2,5\}$ group, and so $3 \| \boldsymbol{A}_{\mathfrak{m}}(\mathfrak{B}) \mid$. Thus, $\mathfrak{K}_{0} \mathfrak{B} / \mathfrak{S}_{0} \mathfrak{B}$ is a chief factor of $\mathfrak{M}$. Let $\mathfrak{W}$ be a $S_{2,3}$-subgroup of $\boldsymbol{A}_{\mathfrak{M}}\left(\mathfrak{B} / \mathfrak{B}^{\prime}\right)$, where $\mathfrak{W} \supseteq \boldsymbol{A}_{\mathfrak{x}}\left(\mathfrak{B} / \mathfrak{B}^{\prime}\right)=\mathfrak{W}_{2}$. Thus, $\mathfrak{W}_{2}$ is a $S_{2}$-subgroup of $\mathfrak{B}$, and $\mathfrak{W}$ may be identified with a subgroup of $G L\left(\mathfrak{B} / \mathfrak{B}^{\prime}\right)$, and $\mathfrak{A P B}^{\prime} / \mathfrak{B}^{\prime}$ is a 1-dimensional subspace of $\mathfrak{B} / \mathfrak{B}^{\prime}$ which is centralized by $\mathfrak{W}_{2}$. This implies that $\mathfrak{W}_{2}$ is cyclic of order 2 or 4 . Since 3|| $\mathfrak{W} \mid$, it follows that $\mathfrak{W} \cong \Sigma_{3}$.

Since $\mathfrak{F}_{0} \mathfrak{B} / \mathfrak{S}_{0} \mathfrak{B}^{\prime}$ is a chief factor of $\mathfrak{M}$, it follows that $[\mathfrak{R}, \mathfrak{B}]=\mathfrak{B}^{\prime}$, and so $\beta(\mathrm{i})$ holds.

Since $3 \notin N$, it follows that $J_{1}(\mathfrak{T}) \nsubseteq \mathfrak{F}_{\mathfrak{C}}$, and as we have seen, $|\mathfrak{T}: \mathfrak{S}|=2$. Hence, $\mathfrak{T}=\mathfrak{S} \mathscr{Z}$, where $\mathfrak{F}^{\prime}=1$ and $m(\mathfrak{F}) \geqslant d(\mathfrak{T})-1$.

Let $\mathfrak{Q}$ be a $S_{3}$-subgroup of $\mathfrak{M}$ which is permutable with $\mathfrak{I}$ and with $\mathfrak{R}$. Let $\mathfrak{M}^{*}=\mathfrak{T} \mathfrak{R} \Omega$. Then $\mathfrak{S}$ is a $S_{2}$-subgroup of $\boldsymbol{O}_{5^{\prime}}\left(\mathfrak{M} \mathbb{R}^{*}\right)$. Let $\Re=N_{\mathfrak{m}^{*}}(\mathfrak{S})=\mathfrak{T} \mathfrak{N} \mathfrak{\Omega}_{0}$, where $\mathfrak{\Omega}_{0} \subseteq \mathfrak{l}$. Then $\mathfrak{S} \mathfrak{B} / \mathfrak{S} \mathfrak{B}^{\prime}$ is a chief factor of $\Omega$. Since $1 \notin N$, it follows that $\Re$ has a minimal normal subgroup (5) such that $C_{\mathfrak{B}}(\mathfrak{F}) \subseteq \mathfrak{B}^{\prime}$. Since $\mathfrak{S} \mathfrak{B} \triangleleft \mathfrak{R}$, we have $\mathbb{F}=[\mathfrak{F}, \mathfrak{B}]$. Choose $f \in \mathfrak{F}-\mathfrak{S}$, and let $x \in \mathfrak{R}-\mathfrak{B}^{\prime}$ be chosen so that $f$ inverts $x \mathfrak{S}$. Since $m(\mathfrak{F}) \geqslant d-1$, it follows that $\left|\mathfrak{s}: C_{\mathbb{E}}(f)\right| \leqq 4$. This implies that $[\mathfrak{F},\langle x\rangle]=\mathfrak{F}_{x}$ is of order $2^{4}$. Let $y$ be a 3-element of $N_{\Re}(\mathfrak{B})$ chosen so that $\mathfrak{B}=\left\langle x, x^{y}\right\rangle$. Thus, $\mathfrak{F}_{0}=\left\langle\mathfrak{F}_{x}, \mathfrak{F}_{x} y\right\rangle$ is of order $₹ 2^{8}$ and $\mathfrak{B}$ centralizes $\mathbb{E} / \mathbb{F}_{0}$. Since $\mathbb{F}=[\mathfrak{F}, \mathfrak{B}]$ we get $\mathbb{F}=\mathbb{F}_{0}$. Thus, $|\mathbb{F}|=2^{\text {, }}$, $\mathfrak{B}^{\prime} \subseteq C(\mathfrak{F})$, and precisely 2 subgroups of $\mathfrak{B} / \mathfrak{B}^{\prime}$ of order 5 have non trivial fixed points on $\mathfrak{F}^{5}$. This is impossible since 3|| $\boldsymbol{A}_{\Omega}\left(\mathfrak{B} / \mathfrak{B}^{\prime}\right) \mid$.

Case $\gamma . \quad \Re^{\prime}=1$.

Case $\gamma(\mathrm{i}) . \quad 3|| \boldsymbol{A}_{\mathfrak{n}}(\Re) \mid$.

Since $\boldsymbol{A}_{\mathfrak{n}}(\mathfrak{R}) \cong \boldsymbol{A}_{\mathfrak{n}}(\mathfrak{B})$, where $\mathfrak{B}=\Omega_{1}(\mathfrak{R})$, and since $\boldsymbol{A}_{\mathfrak{x}}(\mathfrak{B})$ centralizes 
$\mathfrak{A}$, it follows that $\boldsymbol{A}_{\mathfrak{M}}(\Re) \cong \Sigma_{3}$, and so $\mathfrak{S} \mathfrak{B} / \mathfrak{S}$ is a chief factor of $N_{\mathfrak{M}}\left(S_{\mathfrak{C}}\right)$. The preceding argument may now be used to show that $J_{1}(\mathfrak{S}) \leqq \mathfrak{S}_{\mathcal{E}}$, so that $|N| \geqslant 2$. Thus, this case does not arise.

Case $\gamma\left(\right.$ ii). $\quad 3 \nmid\left|\boldsymbol{A}_{\mathfrak{R}}(\Re)\right|$.

In this case, it follows that $\boldsymbol{A}_{\mathfrak{m}}(\Re)=\boldsymbol{A}_{\mathfrak{r}}(\mathfrak{R})$, and so $\mathfrak{R} \nsubseteq \mathfrak{M}^{\prime}$. Hence, $\boldsymbol{A}_{\mathfrak{N}}(\Re) \subset \boldsymbol{A}_{\circledast}(\Re)$. We argue that $\boldsymbol{A}_{\mathfrak{R}}(\Re)$ is a $S_{2}$-subgroup of

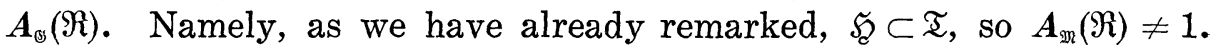
Hence, $\mathfrak{X}$ is the only subgroup of $\mathfrak{R}$ of order 5 which is centralized by $\boldsymbol{A}_{\mathfrak{M}}(\Re)$. Since $\boldsymbol{N}_{\boldsymbol{A}_{\mathfrak{G}}(\mathfrak{R})}\left(\boldsymbol{A}_{\mathfrak{M}}(\Re)\right)$ normalizes $\mathfrak{A}$, and since $\boldsymbol{N}(\mathfrak{M}) \subseteq \mathfrak{M}$, we get that $\boldsymbol{A}_{\mathfrak{N}}(\Re)$ is self normalizing in $\boldsymbol{A}_{\mathscr{S}}(\Re)$. This implies that $\left|\boldsymbol{A}_{\mathfrak{R}}(\Re)\right|=$ $2, \boldsymbol{A}_{\circledast}(\Re) \cong \Sigma_{3}$.

Let $\mathscr{R}$ be the set of all subgroups of $\Re$ of order 5 which have non trivial fixed points on $\mathfrak{S}$. Let $X$ be an element of $\boldsymbol{A}_{\mathbb{S}}(\Re)$ of order 3. Hence, $\mathscr{R} \cap \mathscr{R}^{x}=\varnothing, \mathscr{R} \cap \mathscr{R}^{x^{-1}}=\varnothing$, by Lemma 14.9. Since $\mathfrak{B}$ has exactly 6 subgroups of order 5 , we get $|\mathscr{R}| \leqq 3$. If $|\mathscr{R}|=3$, then we get $|\mathscr{R}|^{x}=|\mathscr{R}|^{X^{-1}}$, so that $\mathscr{R}=\mathscr{R}^{x}$, as $X$ has order 3. This is absurd, so $|\mathscr{R}| \leqq 2$. Since $\mathfrak{B}$ acts faithfully on $\mathfrak{S}_{\mathrm{S}}$, we get $|\mathscr{R}|=2, \mathscr{R}=\left\{\Re_{1}, \Re_{2}\right\}$.

By Lemma 5.58, it follows that $\mathfrak{S}_{\mathfrak{Z}}=\mathfrak{S}_{1} \times \mathscr{S}_{2}$, where $\mathfrak{S}_{i}=C_{\mathfrak{F}}\left(\Re_{i}\right)$. Since $\mathfrak{Q} \in \mathscr{R}$, we choose notation so that $\mathfrak{A}=\mathfrak{R}_{1}$. Since $\mathscr{R}$ admits $\boldsymbol{A}_{\mathfrak{n}}(\Re)$, it follows that $\boldsymbol{A}_{\mathfrak{n}}(\Re)$ normalizes $\Re_{2}$. Thus, $\boldsymbol{A}_{\mathfrak{n}}(\Re)=\langle U\rangle$, where $U$ inverts $\Re_{2}$. Also, $\mathfrak{S}_{i} \triangleleft \mathfrak{T} \Re, i=1,2$, since $\mathfrak{R}_{i} \triangleleft N_{\mathfrak{M}}(\Re), i=$ 1,2 , and since $\mathfrak{S}_{i} \triangleleft \mathfrak{S}_{\mathfrak{C}}$.

Since $\boldsymbol{C}_{\mathfrak{r}}(\boldsymbol{Z}(\mathfrak{T}))=1$, it follows that $\boldsymbol{J}(\mathfrak{T}) \triangleleft \mathfrak{I} \Re$. Hence, $\boldsymbol{J}(\mathfrak{T}) \pitchfork \mathfrak{S}$. This implies that $\mathfrak{Q}$ centralizes $\boldsymbol{Z}(\mathfrak{T})$. Hence, $\mathfrak{Q}$ centralizes $\mathfrak{S}_{2} \cap \boldsymbol{Z}(\mathfrak{T}) \neq 1$. Hence, $\left\langle\mathfrak{R}_{2}, \mathfrak{I}, \mathfrak{Q}\right\rangle \subseteq \boldsymbol{C}\left(\mathfrak{S}_{2} \cap \boldsymbol{Z}(\mathfrak{T})\right)$, so that $\left\langle\mathfrak{R}_{2}, \mathfrak{I}, \mathfrak{Q}\right\rangle$ is solvable. Thus, $\left\langle\mathfrak{R}_{2}, \mathfrak{T}, \mathfrak{Q}\right\rangle=\widetilde{\Phi} \in \mathscr{S}$. Also, $|\widetilde{S} \cap \mathfrak{M}|_{2}=|\mathfrak{I}|=|\mathfrak{S} \cap \mathfrak{M}|_{2}$, and $|\widetilde{\mathfrak{S}}|_{2}=|\mathfrak{I}|=|\mathfrak{S}|_{2}$. For each prime $t$ in $\pi(\tilde{S})$, we may repeat all the above arguments with a suitable $S_{2,5, t}$-subgroup of $\widetilde{\Phi}$ in the role of S. We conclude that $\mathfrak{I} \triangleleft \mathfrak{I R}_{2}$. This is not the case, so the proof is complete.

LEMMA 14.26. $|\mathfrak{B}| \leqq 4$.

Proof. Suppose false. We will use Lemma 14.25 to imitate the plan of $\S 13$. that

Let $\hat{\mathfrak{B}}=\left\{V \in \mathfrak{S}^{\sharp}, r\right.$ divides $\left.\left|\boldsymbol{C}_{\mathfrak{m}}(V)\right|\right\}$. By Lemma 14.25, it follows

Since $r \geqslant 5$, it follows that 
(14.3) if $\mathfrak{W}$ is a subgroup of $\mathfrak{B}$ of index at most 4 , then $\mathfrak{W} \cap \hat{\mathfrak{B}} \neq \varnothing$, so in particular,

(14.4) $\quad C(\mathfrak{W}) \subseteq \mathfrak{M}$ for all subgroups $\mathfrak{W}$ of $\mathfrak{B}$ of index at most 4 .

Let $\mathfrak{I}$ be a $S_{2}$-subgroup of $\mathfrak{M}$ which is permutable with $\mathfrak{R}$, and let $\mathscr{S}_{2}$ be a $S_{2}$-subgroup of $\mathbb{S}$ which contains $\mathfrak{T}$. Then $\Omega_{1}\left(Z\left(\mathbb{S}_{2}\right)\right) \subseteq \mathfrak{B}$. If $\left.\Omega_{1}\left(Z \mathscr{G}_{2}\right)\right) \cap \hat{\mathfrak{B}} \neq \varnothing$, we get $\mathfrak{I}=\mathbb{S}_{2}$, by (14.2). Suppose

$$
\Omega_{1}\left(\boldsymbol{Z}\left(\mathbb{S}_{2}\right)\right) \cap \hat{\mathfrak{B}}=\varnothing .
$$

In this case, $\Re$ acts faithfully on $\mathfrak{B}$. Let $\Re_{0}=\Re \cap C(Z(\mathfrak{T}))$. Then $\mathfrak{R}_{0} \mathfrak{T}$ is a group, since $\mathfrak{S}_{0} \mathfrak{R} \triangleleft \mathfrak{I} \Re$. Let $\mathfrak{V}_{1}=\left[\mathfrak{R}_{0}, \mathfrak{B}\right]$. Thus, since $\mathfrak{B} \subseteq Z\left(\mathfrak{S}_{0}\right)$, it follows that $\mathfrak{B}_{1} \triangleleft \mathfrak{I}$. If $\mathfrak{B}_{1} \neq 1$, then $\mathfrak{B}_{1} \cap \boldsymbol{Z}(\mathfrak{T}) \neq 1$, so $\mathfrak{B}_{1} \cap C\left(\Re_{0}\right) \neq 1$. This is false, and so $\mathfrak{B}_{1}=1$, whence $\mathfrak{R}_{0}=1$, as $\mathfrak{R}$ acts faithfully on $\mathfrak{B}$. By Theorem 1 of [43], it follows that $J(\mathfrak{T}) \triangleleft \mathfrak{T} \Re$. Hence, by Lemma $14.20, N(J(\mathfrak{T})) \subseteq \mathfrak{M}$, and so we have shown that

$$
\mathfrak{T} \text { is a } S_{2} \text {-subgroup of }(S)
$$

We next prove the analogue of Lemma 13.6 which we need:

(14.6) if $\mathfrak{B}_{0}$ is of index 2 in $\mathfrak{B}$ and $I$ is an involution of $\mathfrak{M}$ such that $C_{\mathfrak{B}}(I)=\mathfrak{B}_{0}$, then $[\mathfrak{B}, I]=\langle J\rangle$, where $J \in \mathscr{J}$.

Namely, $\mathfrak{S}_{0}$ centralizes $\mathfrak{B}$, and $\mathfrak{S}_{0} \Re \triangleleft \mathfrak{M}$, so $I$ normalizes $\mathfrak{S}_{0} \Re$. If $I$ does not centralize $\mathfrak{S}_{0} \Re / \mathscr{\mathcal { E }}_{0}$, let $X$ be an element of $\mathfrak{S}_{0} \Re$ of order $r$ which is inverted by $I$. Since $r \geqslant 5$, and since $C_{\mathfrak{B}}(I)$ is a hyperplane of $\mathfrak{B}$, we get that $X$ centralizes $\mathfrak{B}$. Thus, $\mathfrak{B}^{\sharp}=\tilde{\mathfrak{B}} \subseteq \mathscr{I}$, and (14.6) holds. If $I$ centralizes $\mathfrak{\mathcal { S } _ { 0 }} \Re / \mathfrak{K}_{0}$, then $\mathfrak{R}$ normalizes $\langle J\rangle=[\mathfrak{B}, I]$, and so $\Re$ centralizes $J$, whence $J \in \hat{\mathfrak{B}} \subseteq \mathscr{I}$. This is (14.6).

Let $\mathfrak{B}^{*}=V\left(\operatorname{ccl}_{\circledast}(\mathfrak{B}) ; \mathfrak{T}\right)$. We will show that

$$
\mathfrak{B} \subseteq Z\left(\mathfrak{B}^{*}\right) \text {. }
$$

Suppose false. Choose $G$ in $\mathbb{S}$ such that $\mathfrak{X}=\mathfrak{B}^{G} \subseteq \mathfrak{I},[\mathfrak{X}, \mathfrak{B}] \neq 1$. The various portions of Lemma 13.6 which are required in the proof of Lemma 13.7 have been established, and so (14.7) follows.

Next, we see that the analogue of Lemma 13.8 is available:

$$
\mathfrak{M}=C(\mathfrak{B}) \cdot N_{\mathfrak{n}}\left(\mathfrak{B}^{*}\right),
$$

One of the following holds:

(i) $\mathfrak{B}^{*} \subseteq \mathscr{I}$.

(ii) $N_{\mathfrak{M}}\left(\mathfrak{B}^{*}\right) \in \mathscr{L}^{*}(\mathbb{S})$.

Namely, (14.8) is an immediate consequence of (14.7). As for (14.9), if $\Re$ is not faithful on $\mathfrak{B}$, then (14.9) (i) holds by Lemma 14.25. If 
$\Re$ is faithful on $\mathfrak{B}$, then $\Re \subseteq N(\mathfrak{B} *$ ), so (14.9) (ii) holds by Lemma 14.20. Next, we get as in the proof of Lemma 13.9 that

$$
N(\mathfrak{I}) \subseteq \mathfrak{M}
$$

Continuing, we will show that

Lemma 13.10 holds.

Namely, we may assume that $\mathfrak{B}^{\sharp} \nsubseteq \mathcal{F}$, and so we get that $\mathfrak{R}$ is faithful on $\mathfrak{B}$. We may also assume that $\mathfrak{X} \subseteq \mathfrak{I}$, so that $\mathfrak{X}$ normalizes $\mathfrak{F}_{0} \Re$. Suppose $\mathfrak{U}$ acts faithfully on $\mathfrak{S}_{0} \Re / \mathscr{S}_{0}$. By Lemma 5.34 , there is then $A \in \mathfrak{A}^{\#}$ such that $A$ inverts $\mathfrak{B} \mathfrak{S}_{0} / \mathfrak{S}_{0}$, where $\mathfrak{B} \subseteq \Re$, and $\mathfrak{B}$ is elementary of order $r^{2}$. Since $r \geqslant 5$, and since $\mathfrak{B S}_{\mathcal{C}_{0}} / \mathscr{S}_{0}$ acts faithfully on $\mathfrak{B}$, it follows that $\left|\mathfrak{B}: \boldsymbol{C}_{\mathfrak{B}}(A)\right| \geqslant 2^{4}$. This violates the hypothesis that $|\mathfrak{B}: \tilde{\mathfrak{B}}|=2^{2}$. So $\mathfrak{U}^{\sharp}$ contains an element $A$ which centralizes $\mathfrak{S}_{0} \mathfrak{R} / \mathfrak{F}_{0}$. Hence, $\mathfrak{R}$ normalizes $[\mathfrak{B}, A]$, which is of order 2 or 4 , so $\mathfrak{R}$ centralizes $[\mathfrak{B}, A]$. Let $\mathfrak{B}_{0}=\boldsymbol{C}_{\mathfrak{B}}(\mathfrak{X}) \supseteqq \tilde{\mathfrak{B}}$. If $V \in \mathfrak{B}_{0}$, then since $[\mathfrak{B}, A]^{*} \subseteq \hat{\mathfrak{B}},(14.11)$ follows. We may assume that $\mathfrak{B}_{0}$ in a hyperplane of $\mathfrak{B}$ which contains $V$. Since $\tilde{\mathfrak{B}}$ is a hyperplane of $\mathfrak{B}_{0}$, and since $\mathfrak{R}$ normalizes $\mathfrak{B}_{0}$, it follows that $\mathfrak{A}$ centralizes $\mathfrak{S}_{0} \mathfrak{R} / \mathfrak{H}_{0}$. This implies that (14.11) holds, since we may take $A$ to be any element of $\mathfrak{U}^{*}$ which does not centralize $V$.

We have already shown that Lemma 13.11 holds, and the proof of Lemma 13.12 can be carried over verbatim, as it alluded to nothing other than Lemma 5.34.

In order to show that Lemma 13.13 holds, we need a substitute for Lemma 5.20:

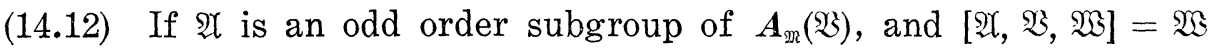
is a 4 -group, then $\mathfrak{W}^{\sharp} \subseteq \mathscr{J}$.

Namely, since $\mathfrak{A} \subseteq \boldsymbol{A}_{\mathfrak{n}}(\mathfrak{B})$, we have $|\mathfrak{A}|=3$. We may assume that $\mathfrak{S}^{\ddagger} \nsubseteq \mathscr{I}$, and so $\mathfrak{R}$ is faithful on $\mathfrak{B}$. Let $\mathfrak{P}$ be a cyclic 3-subgroup of $\mathfrak{M}$ which maps onto $\mathfrak{A}$, and let $\mathfrak{Z}=\mathfrak{S}_{0} \mathfrak{R} \mathfrak{R}$. We assume without loss of generality that $\mathfrak{P}$ normalizes $\mathfrak{R}$. Since $[\mathfrak{P}, \mathfrak{B}]$ is a 4-group and $\boldsymbol{C}_{\mathfrak{R}}(\mathfrak{B})=1$, it follows that $[\mathfrak{P}, \mathfrak{R}]=1$. Hence, $\mathfrak{R}$ centralizes $[\mathfrak{P}, \mathfrak{B}]=$ $\mathfrak{W}$, so (14.12) holds.

In proceeding through the proof of Lemma 13.11, we get that $\mathfrak{Z}$ is elementary of order $3^{y}$. Since $y \geqslant 2$, we get that $3 \in \sigma_{2}$, whence it follows that $3 \in \pi_{2}$. If $\mathfrak{P}$ is any 3 -group with $\mathscr{S} c n_{3}(\mathfrak{P})=\varnothing$, it is easy to check that every 2-subgroup of Aut( $\mathfrak{B})$ is generated by 2 elements, this being true for every 2-subgroup of $G L(2,3)$. Hence, we conclude that $y=2$.

Since $\mathfrak{2}$ is elementary of order $3^{2}$, it follows that $\mathfrak{Y}$ centralizes the $S_{3^{\prime}}$-subgroup of $\boldsymbol{F}(\mathfrak{M} / \boldsymbol{C}(\mathfrak{B}))$. In particular, $\mathfrak{Y}$ centralizes $\mathfrak{R} C(\mathfrak{B}) / C(\mathfrak{B})$, 
and so does $\mathfrak{X}$. Since $\left|\left[\mathfrak{B}, \mathfrak{N}_{1}\right]\right|=2^{4}$, and since $\left[\mathfrak{B}, \mathfrak{N}_{1}\right]$ admits $\mathfrak{R}$, it follows that $\left[\mathfrak{B}, \mathfrak{N}_{1}\right]^{\sharp} \subseteq \hat{\mathfrak{B}} \cong \mathscr{J}$. Hence, as $\mathfrak{X}_{0}$ is of index $2^{3}$ in $\mathfrak{B}^{G}$, it follows that $\mathfrak{X}_{0} \cap \mathscr{I}^{G} \neq \varnothing$, and so $\boldsymbol{C}\left(\mathfrak{X}_{0}\right) \subseteq \mathfrak{M}^{G}$. Thus, $\mathfrak{B} \subseteq \mathfrak{M}^{G}$, and so $\left[\mathfrak{B}, \mathfrak{B}^{G}\right]=1$, against $\mathfrak{X}=\mathfrak{W} \cap \mathfrak{B}^{G} \subset \mathfrak{B}^{G}$. We have thus shown that

$$
\begin{aligned}
& \text { if } G \in \mathbb{S} \text { and }\left|\mathfrak{B}^{G}: \mathfrak{B}^{G} \cap \mathfrak{M}\right| \leqq 2 \text {, then } \mathfrak{B}^{G} \subseteq \mathfrak{M} \text {. } \\
& \text { Next, we proceed to show that } \\
& \text { either } N\left(\mathfrak{B}^{*}\right) \subseteq \mathfrak{M} \text { or } \mathfrak{M}=N_{\mathfrak{M}}\left(\mathfrak{B}^{*}\right) \cdot N_{\mathfrak{M}}\left(\mathfrak{W}_{0}\right) \text {, where } \\
& \mathfrak{W}_{0}=Z\left(\mathfrak{W}_{1}\right) \text { and } \mathfrak{M}_{1}=\langle\mathfrak{U}| \mathfrak{U} \subseteq \mathfrak{I} \text { and for some } \\
& \left.G \text { in } \mathfrak{S}, \mathfrak{U} \text { is of index } 2 \text { in } \mathfrak{B}^{G}\right\rangle \text {. }
\end{aligned}
$$

Namely, if $N\left(\mathfrak{B}^{*}\right) \nsubseteq \mathfrak{M}$, then $r|| \boldsymbol{C}(\mathfrak{B}) \mid$, and so $\mathfrak{B}^{\sharp} \subseteq \mathscr{J}$. We can now follow the proof of Lemma 13.16 to obtain (14.14). Lemma 13.17 now follows, and we must establish Lemma 13.18. We need to show that $\boldsymbol{N}\left(\mathfrak{W}_{0}\right) \subseteq \mathfrak{M}$ provided $N\left(\mathfrak{B}^{*}\right) \nsubseteq \mathfrak{M}$. Let $\mathfrak{R}_{1}=\boldsymbol{N}_{\mathfrak{\Re}}\left(\mathfrak{W}_{0}\right), \mathfrak{R}_{2}=\boldsymbol{N}_{\mathfrak{r}}\left(\mathfrak{B}^{*}\right)$, so that $\Re_{1} \Re_{2}=\Re$. Thus, $\Re_{2}$ is cyclic, by Lemma 14.20 , together with the assumption that $N\left(\mathfrak{B}^{*}\right) \nsubseteq \mathfrak{M}$. We may assume that $\Re_{1}$ is also cyclic, since otherwise Lemma 14.20 implies that $N\left(\mathfrak{W}_{0}\right) \subseteq \mathfrak{M}$. Now $\mathfrak{W}_{1}$ is generated by subgroups $\mathfrak{U}$ which contained in $\mathfrak{I}$ and are hyperplanes of $\mathfrak{B}^{G}$. By (14.13), we get that $\mathfrak{B}^{G} \leqq \mathfrak{M}$, and so $\left[\mathfrak{B}^{G}, \mathfrak{B}\right]=$ $[\mathfrak{U}, \mathfrak{B}]=1$, so $\mathfrak{B}^{*} \subseteq \boldsymbol{C}\left(\mathfrak{W}_{0}\right)$. If $r|| \boldsymbol{C}_{\mathfrak{n}}\left(\mathfrak{W}_{0}\right) \mid$, then $\mathfrak{W}_{0}^{*} \subseteq \hat{\mathcal{J}} \subseteq \mathscr{J}$, and by Lemma 14.25 , we get $C\left(\mathfrak{B}_{0}\right) \in \mathfrak{M}^{*}(\mathbb{S})$, whence $N\left(\mathfrak{W}_{0}\right) \subseteq \mathfrak{M}$. We may therefore assume that $r \nmid\left|C_{\mathfrak{m}}\left(\mathfrak{W}_{0}\right)\right|$. Hence, $\Re=\Re_{1} \Re_{2} \subseteq N\left(\mathfrak{B}^{*}\right)$, against $N\left(\mathfrak{B}^{*}\right) \nsubseteq \mathfrak{M}$. This gives us Lemma 13.18, and the proof of Lemma 13.19 suffices to complete a proof that $|\mathfrak{B}| \leqq 4$.

\section{THEOREM 14.1. $\sigma_{2}=\{3\}$.}

Proof. Suppose false, so that $r \geqslant 5$ and Hypothesis 14.2 is satisfied. We retain the earlier notation.

If every normal abelian subgroup of $\mathfrak{M}$ is cyclic, then Theorems $13.5,13.6$, and 13.7 yield a contradiction. Suppose every normal abelian subgroup of $\mathfrak{M}$ is generated by 2 elements and that $\mathfrak{A}$ is a non cyclic normal abelian subgroup of $\mathfrak{M}$. Since $r \geqslant 5, \mathfrak{R}$ centralizes $\mathfrak{A}$, and so every involution of $\mathscr{A}$ is in $\mathscr{F}$, by Lemma 14.25. This violates Theorem 13.4. Hence, $\mathfrak{M}$ contains a normal elementary abelian 2subgroup of order $\geqslant 8$. Let $₹$ be such a subgroup of minimal order. By Lemma $14.26, \mathfrak{F} \nsubseteq \mathfrak{B}$, and so $\mathfrak{F}$ is not a minimal normal subgroup of $\mathfrak{M}$. Let $\mathfrak{F} / \mathfrak{F}$ be a chief factor of $\mathfrak{M}$. By minimality of $\mathfrak{F}$, we get $2 \leqq|\mathfrak{F}| \leqq 2^{2}$. Thus, $\Re$ centralizes $\mathfrak{F}$. Let $\mathfrak{F}=\boldsymbol{C}(\mathfrak{F}), \mathfrak{M}_{0}=$ $\boldsymbol{C}_{\mathfrak{n}}(\mathfrak{F}), \mathfrak{D}=\boldsymbol{O}_{2}\left(\mathfrak{M}_{0} \bmod \mathfrak{S}\right)$.

Let $\hat{\mathfrak{M}}$ be the set of elements of $\mathfrak{M}$ of order $r$. Let $\mathfrak{T}$ be a $S_{2^{-}}$ subgroup of $\mathfrak{M}$ permutable with $\Re$. 
We will use the preceding results of this section to follow the plan of $\S 13$. However, this is not completely straightforward, so we proceed carefully. We need the analogues of Lemmas 13.26 through 13.28 .

By Lemma 14.25, we have

$$
\text { if } \begin{aligned}
I \in \mathfrak{F}^{\sharp} \text { and } C_{\mathfrak{M}}(I) \cap \hat{\mathfrak{M}} \neq \varnothing, \text { then } I \in \mathscr{F} \text {. } \\
\qquad \mathfrak{F} \cong \mathscr{\mathcal { F }} .
\end{aligned}
$$

Note that (14.15) is weaker than (a) of Lemma 13.26, but it will suffice for our purposes.

If $\Re$ is not faithful on $\mathfrak{F}$, then $\mathfrak{F}^{*} \subseteq \mathscr{I}$. If $\Re$ is faithful on $\mathfrak{F}$, then since $r \geqslant 5$, and since $\widetilde{F} \neq 1$, it follows that (c) (ii) of Lemma 13.26 holds. Hence,

$$
\text { One of the following holds: }
$$

(i ) $\mathfrak{F}^{\#} \cong \mathscr{F}$.

(ii) $\widetilde{F}$ is generated by subgroups $\mathfrak{U}$ of order 16 such that $\mathfrak{2} \mathfrak{l} \subseteq \mathscr{I}$.

The proof of Lemma 13.27 carries over verbatim, and the analogue of Lemma 13.28 is

One of the following holds:

(a) $\mathfrak{\mho}^{\sharp} \subseteq \mathscr{F}$.

(b) $\boldsymbol{C}_{\mathfrak{n}}(\mathfrak{F})$ is an $r^{\prime}$-group.

We turn to the proof of the crucial Lemma 13.29. We must be content with a more elaborate statement.

(14.19) Suppose $J$ is an involution of $\mathfrak{M}-C_{\mathfrak{M}}(\mathfrak{F})$. Let $\mathfrak{F}_{0}=C_{\widetilde{\delta}}(J)$, $2^{f}=\left|\mathfrak{F}: \mathfrak{\mho}_{0}\right|$.

(a) If $\left[\mathfrak{F} \nsubseteq \mathfrak{F}_{0}\right.$, then $[\mathfrak{F}, J] \cap \mathscr{J} \neq \varnothing$.

(b) Suppose $\mathfrak{F} \subseteq \mathfrak{F}_{0}$.

(i) If $f \leqq 2$, then $[\mathfrak{F}, J]^{*} \subseteq \mathscr{F}$.

(ii) If $f=3$, then one of the following holds:

$(\alpha)[\widetilde{F}, J]^{\sharp} \subseteq \mathscr{I}$.

(ß) $\quad r=7,|\Re|=7^{2}$, and the image of $\Re$ in $\boldsymbol{A}_{\mathfrak{n}}(\mathfrak{F} / \mathfrak{S})$ is a self centralizing normal subgroup of $\boldsymbol{A}_{\mathfrak{n}}(\mathfrak{F} / \mathfrak{F})$. Furthermore, $|\mathfrak{F} / \mathfrak{F}|=2^{3 k}$, where $k=2$ or 3 .

Namely, (14.19) (a) is a consequence of (14.16). From now on, we assume that $\mathfrak{F} \subseteq \mathfrak{F}_{0}$ and that $f \leqq 3$.

Since $\mathfrak{S}_{0} \Re \triangleleft \mathfrak{M}$, it follows that $J$ acts on $\mathscr{H}_{0} \Re / \mathscr{F}_{0}$, and since $\mathfrak{F}_{0}=\boldsymbol{O}_{2}(\mathfrak{M})$, we have $\left[\mathfrak{F}_{0}, \mathfrak{F}\right] \subseteq\left(\mathfrak{F}\right.$. Let $\Re_{1}$ be the subgroup of $\Re$ such that $\Re_{1} \mathfrak{S}_{0} / \mathfrak{F}_{0}=C_{\Re \mathfrak{F}_{0} / \mathfrak{F}_{0}}(J)$. Then $\Re_{1}$ normalizes $[\mathfrak{F}, J]$ [r. If $C_{\Re_{1}}([\mathfrak{F}, J]) \neq 1$, 
then $[\widetilde{F}, J]^{\sharp} \cong \mathscr{I}$, and (14.19) (b) (ii) ( $\alpha$ ) holds if $f=3$ and (14.19) (b) (i) holds if $f \leqq 2$. We may assume that $C_{\Re_{1}}([\mathfrak{F}, J] \mathfrak{F})=1$. We may also assume that $\Re$ acts faithfully on $\mathfrak{F}$. Since $r \geqslant 5$ and $f \leqslant 3$, it follows that $J$ does not invert $\Re \mathfrak{S}_{0} / \mathfrak{S}_{0}$, and so $\Re_{1} \neq 1$. Since $\Re_{1}$ centralizes $\mathfrak{F}$, it follows that $\Re_{1}$ acts faithfully on [₹, $\left.J\right] \mathfrak{F} / \mathfrak{F}$. Hence, $r=7$. This implies that $f=3$, so that (14.19) (b) (i) holds.

Let $\Re_{2}$ be a subgroup of $\mathfrak{S}_{0} \Re$ of order 7 which is inverted by $J$. We assume without loss of generality that $\Re_{2} \subseteq C_{\Re}\left(\Re_{1}\right)$. Since $f=3$, it follows that $\left[\mathfrak{F}, \Re_{2}\right]=\mathfrak{F}^{\circ}$ is of order $2^{6}$, and $\mathfrak{F}^{\circ} \supseteqq[\mathfrak{F}, J]$. Thus, $\boldsymbol{C}_{\Re}\left(\Re_{2}\right)$ acts on $\mathfrak{F}^{0}$, and if $\boldsymbol{C}_{\Re}\left(\Re_{2}\right) \supset \Re_{1} \Re_{2}$, then $\boldsymbol{C}_{\Re}\left(\mathfrak{F}^{\circ}\right) \neq 1$, which gives $\mathfrak{F}^{0 *} \subseteq \mathscr{F}$, so that (14.19) (b) (ii) ( $\alpha$ ) holds. Hence, we may assume that $C_{\Re}\left(\Re_{2}\right)=\Re_{1} \Re_{2}$. If $\Re_{1} \Re_{2} \subset \Re$, let $\Re^{*}$ be a subgroup of $\Re$ of order $7^{3}$ which contains $\Re_{1} \Re_{2}$. Since $\Re^{*}$ is faithful on $\mathfrak{F}$, and since $\Re^{*}$ is non abelian, it follows that $\left|\left[\Re_{2}, \mathfrak{\Im}\right]\right| \geqslant 7^{18}$. This is false, and so $\Re=$ $\Re_{1} \times \Re_{2}$ is of order $7^{2}$.

Since $\mathfrak{F}^{\circ}$ admits $\Re$, it follows that $\Re$ is faithful on $\mathfrak{F}^{\circ}$. Let $\Re$ be a $S_{2^{\prime}}$-subgroup of $\boldsymbol{O}_{r^{\prime}}(\mathfrak{M})$ which admits $\Re$. Then $R$ centralizes $\Re$, and so $\Re$ normalizes $\mathfrak{F}^{0}$. Since $S_{7}$-subgroups of $G L(6,2)$ are self centralizing, it follows that $\Re$ centralizes $\mathfrak{F}^{0}$. Since $\mathfrak{S} \Re \triangleleft \mathfrak{M}$, and since $\mathfrak{F} / \mathfrak{F}$ is a chief factor of $\mathfrak{M}$, it follows that $\Re$ centralizes $\mathfrak{F} / \mathfrak{F}$.

Let $\mathfrak{L}=\boldsymbol{A}_{\mathfrak{n}}(\mathfrak{F} / \mathfrak{F})$, let $\mathfrak{R}_{7}$ be the image of $\mathfrak{R}$ in $\mathfrak{L}$, so that $\mathfrak{R} \cong$ $\mathfrak{L}_{7} \triangleleft \mathfrak{L}$. Furthermore, $\boldsymbol{O}_{2}(\mathfrak{R})=1$, since $\mathfrak{F} / \mathbb{F}$ is a chief factor of $\mathfrak{M}$. Hence, the Fitting subgroup of $\mathbb{Q}$ has odd order, and so $\boldsymbol{O}_{7^{\prime}}(\mathfrak{F}(\mathbb{R}))=1$, as $\Omega$ maps onto $\boldsymbol{O}_{7}(\boldsymbol{F}(\mathfrak{Q}))$. Thus, (14.19) (b) (ii) $(\beta)$ holds, provided we can show that $\mid \Im /(F)=2^{6}$ or $2^{9}$.

If $\Re_{2} \triangleleft N_{\mathfrak{n}}(\Re)$, then $N_{\mathfrak{n}}(\Re)$ normalizes $\mathfrak{F}^{0}=\left[\mathfrak{F}, \Re_{2}\right]$, and since $\mathfrak{F} / \mathfrak{F}$ is a chief factor of $\mathfrak{M}$ and $\mathfrak{M}=C_{\mathfrak{M}}(\mathfrak{F} / \mathfrak{F}) \cdot N_{\mathfrak{M}}(\mathfrak{R})$, we get $|\mathfrak{F} / \mathfrak{F}|=2^{6}$. Suppose $\Re_{2} \rtimes N_{\mathfrak{n}}(\Re)$. Choose $M \subseteq N_{\mathfrak{m}}(\mathfrak{F}), M \notin N\left(\Re_{2}\right)$, and set $\Re^{2}=\Re_{2}^{M}$. Thus, $\Re=\Re_{2} \times \Re^{2}$. Since $\mathfrak{F} / \mathbb{E}$ is a chief factor of $\mathfrak{M}$ and $\mathfrak{R} \cong \mathfrak{L}_{7} \triangleleft \mathfrak{R}$, it follows that $\Re$ has no fixed points on $\mathfrak{F} / \mathfrak{F}$. Hence, $\mathfrak{F}=\mathfrak{F} \cdot \mathfrak{F}^{0} \cdot \mathfrak{F}^{\circ M}$, and so $|\mathfrak{F} / \mathfrak{F}|=2^{3 k}$, where $2 \leqq k \leqq 4$.

Suppose $k=4$ so that $\mathfrak{F}=\mathfrak{F} \times \mathfrak{F}^{0} \times \mathfrak{F}^{0 . M}$. Since $\left[\mathfrak{R}^{2}, \mathfrak{F}\right]=\mathfrak{F}^{0 . M}$, and since $\mathfrak{F}^{0}$ admits $\Re$, it follows that $\left[\mathfrak{F}^{0}, \Re^{2}\right]=1$. Thus, by Lemma 14.25, we have $\mathfrak{F}^{0 \ddagger} \subseteq \mathscr{F}$, and so (14.19) (b) (ii) $(\alpha)$ holds. This is (14.19).

Next, we need the analogue of Lemma 13.30:

Suppose $G \in \mathbb{S}$ and $\mathfrak{F}^{G} \subseteq \mathfrak{M}$. Then $\mathfrak{F}^{G} \subseteq \mathfrak{E}$.

Suppose false. We argue that there is an involution $J$ of $\mathfrak{M}$. such that $[\mathfrak{F}, J]$ is of order 8 , and $[\mathfrak{F}, J]^{\sharp} \nsubseteq \mathscr{F}$. If this is not the case, then the possibility (14.19) (b) (ii) $(\beta)$ does not trouble us, and we may copy the proof of Lemma 13.30. Thus, we may assume that (14.19) (b) (ii) $(\beta)$ occurs for some $J$. 
Let $\mathfrak{F}^{*}=\mathfrak{F}^{G}, \mathfrak{M}^{*}=\mathfrak{M}^{G}, \mathfrak{F}^{*}=\mathfrak{F}^{*}, \mathfrak{D}^{*}=\mathfrak{D}^{a}, \mathscr{I}^{*} \cap \mathscr{J}^{a}$. We assume that $\left[\mathfrak{F}, \mathfrak{F}^{*}\right] \neq 1$. Hence, $\mathfrak{M} \neq \mathfrak{M}^{*}$, and so $\mathscr{J} \cap \mathscr{J}^{*}=\varnothing$.

As in the proof of Lemma 13.20 , we get $\mathfrak{F}^{*} \nsubseteq \mathfrak{D}$. We also get that $\mathfrak{F}^{*}$ centralizes $\mathfrak{F}$. Since $\mathfrak{R D} / \mathfrak{D}$ is a self centralizing normal subgroup of $\mathfrak{M}_{0} / \mathfrak{D}$ of order $7^{2}$, it follows that $\mathfrak{F}^{*}=\left(\mathfrak{F}^{*} \cap \mathfrak{D}\right) \times \mathfrak{Y} *$, where $\mathfrak{Y} *$ acts faithfully on $\Re D / D$. If some element of $\mathfrak{Y}^{*}$ inverts $\Re \mathfrak{R} / \mathfrak{D}$, it follows that $\mathfrak{F} / \mathfrak{F}$ has order at least $2^{12}$, aginst $|\mathfrak{F} / \mathfrak{F}| \leqq 2^{9}$. So no element of $\mathfrak{Y}^{*}$ inverts $\mathfrak{R D} / \mathfrak{D}$, and so $\mathfrak{Y}^{*}=\left\langle Y^{*}\right\rangle$ is of order 2 .

Since $|\mathbb{F}| \leqq 2^{2}$, it follows that if $F \in \mathfrak{F}$, then $C_{\widetilde{\mho}^{*}}(F)$ is of index at most $2^{3}$ in $\mathfrak{F}^{*}$, and so by (14.17), we get $\mathfrak{F} \subseteq \mathfrak{M}^{*}$.

Let $\mathfrak{F}_{1}^{*}=\mathfrak{F}^{*} \cap \mathfrak{D}$, and let $\mathfrak{F}_{1}=\mathfrak{F} \cap \mathfrak{D}^{*}$. By symmetry, $\left|\mathfrak{F}: \mathfrak{F}_{1}\right|=$ 2. Since $\left[\mathfrak{F}_{1}^{*}, \mathfrak{F}_{1}\right] \subseteq \mathfrak{F} \cap \mathfrak{F}^{*}$, and since $\mathscr{F} \cap \mathscr{J}^{*}=\varnothing$, we have $\left[\mathfrak{F}_{1}^{*}, \mathfrak{F}_{1}\right]=1$. Choose $Y \in \mathfrak{F}-\mathfrak{F}_{1}$. Then

$$
\begin{aligned}
& {\left[\mathfrak{F}^{*}, Y\right]=\left\langle\left[Y^{*}, Y\right]\right\rangle \times\left[\mathfrak{F}_{1}^{*}, Y\right]=\left\langle\left[Y^{*}, Y\right]\right\rangle \times \mathfrak{F},} \\
& {\left[\mathfrak{F}, Y^{*}\right]=\left\langle\left[Y, Y^{*}\right]\right\rangle \times\left[\mathfrak{F}_{1}, Y^{*}\right]=\left\langle\left[Y, Y^{*}\right]\right\rangle \times \mathfrak{F} * .}
\end{aligned}
$$

This is so, since $\left|\left[\mathfrak{F}, Y^{*}\right]\right| \geqslant 2^{3}$, and since $\left[\mathscr{F}_{1}, Y^{*}\right] \subseteq \Im^{*}$. In particular, we have $|\mathfrak{F}|=2^{2}$. Let $\mathfrak{u}=\left[\mathfrak{F}, \mathfrak{F}^{*}\right]=\mathfrak{F} \times\left[\mathfrak{F}^{*} \times\left\langle\left[Y, Y^{*}\right]\right\rangle\right.$, so that $\mathfrak{U} \cong \mathfrak{F} \cap \mathfrak{F}^{*}$, and $|\mathfrak{H}|=2^{5}$. Let $\mathfrak{R}_{1}$ be subgroup of $\mathfrak{R}$ such that

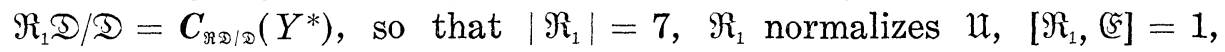
and $\Re_{1}$ is transitive on $(\mathfrak{U} / \mathfrak{G})^{*}$. By symmetry, $N(\mathfrak{U})$ has an element of order 7 which centralizes $\mathfrak{F}^{*}$ and is transitive on $\left(\mathfrak{U} / \mathfrak{F}^{*}\right)^{*}$. Let $\mathfrak{L}=\boldsymbol{N}(\mathfrak{u}) / C(\mathfrak{u})$. Since the only subgroups of $\mathfrak{u}$ which admit $\mathfrak{R}_{1}$ are either contained in $\mathfrak{F}$ or contain $\left[\mathfrak{M}, \mathfrak{R}_{1}\right]$, symmetry implies that $\mathbb{Z}$ acts irreducibly on $\mathfrak{U}$. Since $|\mathfrak{L}|_{7}=7$, it follows that $\mathbb{R}$ is not solvable. This is (14.20).

Here is the analogue of Lemma 13.31:

(14.21) One of the following holds:

(a) $\mathfrak{F}^{\sharp} \subseteq \mathscr{F}$.

(b) $C\left(\mathfrak{F}_{0}\right) \subseteq \mathfrak{M}$ for every subgroup $\mathfrak{F}_{0}$ of index 16 in $\mathfrak{F}$.

(c) $r=7$ and if $\mathfrak{B}$ is any elementary subgroup of $\mathfrak{M}$ of order $7^{2}$, then $C_{\mathfrak{f}}(\mathfrak{B})=\mathbb{F}$ is of order 2 .

Namely, (a) holds if $\Re$ is not faithful on $\mathfrak{F}$. Suppose $\Re$ is faithful on $\widetilde{F}$. If $\left|\boldsymbol{C}_{\widetilde{F}}(M)\right| \geqslant 2^{5}$ for some $M \in \hat{\mathfrak{M}}$, then $\boldsymbol{C}_{\mho_{0}}(M) \neq 1$, and so (b) holds. We may assume that $\left|C_{\Im}(M)\right| \leqq 2^{4}$ for all $M \in \hat{\mathfrak{M}}$. Since $\mathfrak{F} \subseteq \boldsymbol{C}_{\mathscr{F}}(\Re)$, it follows that $\left|\boldsymbol{C}_{\mathscr{\Im} \mid \mathbb{E}}(M)\right| \leqq 16 / \mid[\mathfrak{F} \mid$ for all $M \in \hat{\mathfrak{M}}$. Hence, $|\mathfrak{F}|=2, r=7$ and (c) holds.

We get the precise analogue of Lemma 13.32:

(14.22) One of the following holds:

(a) $\mathfrak{F}^{\sharp} \subseteq \mathscr{F}$.

(b) If $G \in \mathbb{S}$ and $\left|\mathfrak{F}^{G}: \mathfrak{F}^{G} \cap \mathfrak{M}\right| \leqq 2$, then $\mathfrak{F}^{a} \leqq \mathfrak{M}$. 
If (14.21) (a) or (14.21) (b) holds, we may follow the proof of Lemma 13.32 to obtain (14.22), the reference to Lemma 13.29 (b) (i) being replaced by (14.19) (b) (i). So we may assume that (14.21) (c) holds.

Choose $G$ in $\mathfrak{B S}^{3}$ such that $\left|\mathfrak{F}^{G}: \mathfrak{F}^{G} \cap \mathfrak{M}\right|=2$. Let $\mathfrak{M}^{*}=\mathfrak{M}^{G}$, $\mathfrak{F}^{*}=\mathfrak{F}^{G}$, and let $\mathfrak{F}_{1}^{*}=\mathfrak{F}^{*} \cap \mathfrak{M}$. By (14.20), we have $\mathfrak{F} \nsubseteq \mathfrak{M}^{*}$. Hence, $\mathscr{J}^{G} \cap \mathfrak{F}=\varnothing, \mathscr{I}^{*} \cap \mathfrak{F}^{*}=\varnothing$.

Let $\mathfrak{F}_{0}=\mathfrak{F}_{\mathfrak{M}^{*}}$, so that $\mathfrak{F}_{0} \subset \mathfrak{F}$. Choose $F \in \mathfrak{F}-\mathfrak{F}_{0}$. Let $\mathfrak{F}_{2}^{*}=C(F) \cap \mathfrak{F}_{1}^{*}$ and let $2^{f}=\left|\mathfrak{F}_{1}^{*}: \mathfrak{F}_{2}^{*}\right|$. If $f \leqq 2$, then $\left|\mathfrak{F}^{*}: \mathfrak{F}_{2}^{*}\right| \leqq 2^{3}$, so that $\boldsymbol{C}\left(\mathfrak{F}_{2}^{*}\right) \subseteq \mathfrak{M}^{*}$, whence $F \in \mathfrak{M}^{*}$. This is false and so $f \geqslant 3$. Since $|\mathfrak{F}|=2$, if follows that $\mathfrak{\mho}_{1}^{*} \nsubseteq \mathfrak{D}$.

Let $\tilde{\mathfrak{R}} / \mathfrak{D}$ be a subgroup of $\mathfrak{M} / \mathfrak{D}$ of odd prime order which admits $\mathfrak{F}_{1}^{*}$ and is not centralized by $\mathfrak{F}_{1}^{*}$. Let $\mathfrak{F}_{2}^{*}=\mathfrak{F}_{1}^{*} \cap \boldsymbol{C}(\tilde{\Re} / \mathfrak{D})$, so that $\left|\mathfrak{F}_{1}^{*}: \mathfrak{F}_{2}^{*}\right|=2$. Let $\mathfrak{F}_{1} / \mathfrak{F}$ be a subgroup of $\mathfrak{F} / \mathfrak{F}$ which admits $\tilde{R}_{1}^{*}$ and is minimal subject to $\left[\mathfrak{F}_{1}, \tilde{\Re}\right] \nsubseteq \mathfrak{F}$. Then $\left[\mathfrak{F}_{2}^{*}, \mathfrak{\mho}_{1}\right] \subseteq \mathfrak{F}$, an $\mathfrak{F}_{1}^{*}$ does not centralize $\widetilde{F}_{1} / \mathfrak{F}$. Choose $F$ in $\widetilde{F}_{1}$ such that $\mathfrak{F} \widetilde{F}$ is not centralized by $\mathfrak{F}_{1}^{*}$. Since $\left[\mathfrak{F}_{2}^{*}, F\right] \subseteq \mathfrak{F}$, and since $|\mathfrak{F}|=2$, it follows that $\mathfrak{F}_{3}^{*}=$ $\mathfrak{F}_{2}^{*} \cap C(F)$ is of index at most 2 in $\mathfrak{F}_{2}^{*}$, so is of index at most $2^{3}$ in $\mathfrak{F}^{*}$. Hence, $F \in \boldsymbol{C}\left(\mathfrak{F}_{3}^{*}\right) \subseteq \mathfrak{M}^{*}$. Hence, $\left[\mathfrak{F}_{2}^{*}, F\right] \subseteq \mathfrak{F}^{*} \cap F=1$, and so $\mathfrak{F}_{2}^{*}=\mathfrak{F}_{3}^{*}$ is of index 2 in $\mathfrak{F}_{1}^{*}$.

By (14.19) (b) (i) applied with $F$ in the role of $J, \mathfrak{M}^{*}$ in the role of $\mathfrak{M}$, $\mathfrak{F}_{2}^{*}$ in the role of $\mathfrak{F}_{0}, \mathfrak{F}^{*}$ in the role of $\mathfrak{F}$, we get $\left[\mathfrak{F}^{*}, F\right]^{*} \subseteq \mathscr{J}^{G}$. In particular, $\left[\mathfrak{F}_{1}^{*}, F\right]^{\#} \subseteq \mathscr{I}^{G}$. But $1 \subset\left[\mathfrak{F}_{1}^{*}, F\right] \subseteq \mathfrak{F}$, so $\mathscr{I}^{G} \cap \mathfrak{F} \neq \varnothing$. This contradiction gives us (14.22).

The proof of the crucial Lemma 13.33 gives no difficulty, and there is then no difficulty in deriving the final contradiction to complete a proof of Theorem 14.1.

15. The case $\sigma_{2}=\{3\}$. All results of this section are proved on the hypothesis that $2 \in \pi_{4}$ and $\sigma_{2}=\{3\}$. So many of the preceding arguments break down when $r=3$ that it has seemed essential to start afresh. We retain the following notation: $\Re$ is a $S_{3}$-subgroup of $\mathfrak{B S}, \mathfrak{S}$ is a maximal element of $U(\mathfrak{R} ; 2), \mathfrak{M}=\mathfrak{M}(\mathfrak{S}, \mathfrak{R}), \mathfrak{S}_{0}=\boldsymbol{O}_{2}(\mathfrak{M})$, $\mathfrak{I}$ is a $S_{2}$-subgroup of $\mathfrak{M}$ permutable with $\mathfrak{R}, \mathfrak{B}=\Omega_{1}\left(\boldsymbol{R}_{2}(\mathfrak{M})\right)$. Note that $\mathfrak{M}(\mathfrak{K}, \mathfrak{R})$ is well-defined by Lemmas 14.15 through 14.18 .

LEMMA 15.1. If $|\mathfrak{B}|>8$ and $\mathfrak{B}_{0}$ is of index 2 in $\mathfrak{B}$, then $C\left(\mathfrak{B}_{0}\right) \subseteq \mathfrak{M}$.

Proof. Suppose false. Let $\mathfrak{F}_{0}=\boldsymbol{C}\left(\mathfrak{B}_{0}\right) \supseteqq \mathbb{F}=\boldsymbol{C}(\mathfrak{B})$. Since $\mathfrak{M}=$ $N(\mathfrak{B})$, we have $\mathfrak{E}_{0} \supset \mathfrak{E}$. 
Let $\mathfrak{U}$ be a subgroup of $\mathfrak{S}$ of order 3 and let $\mathfrak{B}$ be a subgroup of $\boldsymbol{C}_{\mathfrak{n}}(\mathfrak{V})$ of order 3 with $\mathfrak{B} \neq \mathfrak{N}$. Thus, $\mathfrak{U} \mathfrak{B}$ is elementary of order 9. Let $\mathfrak{B}=\langle B\rangle$, and let $\mathbb{Z}=\left\langle\mathfrak{S}_{0}, \mathfrak{S}_{0}^{B}, \mathfrak{C}_{0}^{B^{2}}\right\rangle$. Then $\mathbb{Q}$ centralizes $\mathfrak{B}_{0} \cap \mathfrak{B}_{0}^{B} \cap \mathfrak{B}_{0}^{B^{2}} \neq 1$, so $\mathbb{Z}$ is solvable. Hence, $\langle\mathfrak{Q}, \mathfrak{B}\rangle=\mathfrak{R} \mathfrak{B}$ is solvable. Since $\mathfrak{S}_{0} \mathfrak{Z} \mathfrak{Z} \subseteq \mathbb{R} \mathfrak{B}$, we get $\mathbb{R} \mathfrak{B} \subseteq \mathfrak{M}$, by Lemma 14.20 .

\section{Case 2. $3 \nmid|\mathfrak{S}|$ and $|\mathfrak{\Im}|_{2}=\left|\mathfrak{\mathfrak { S } _ { 0 }}\right|_{2}$.}

Let $\mathfrak{I}_{0}=\mathfrak{I} \cap \mathfrak{E}$. Thus, $\mathfrak{I}_{0}$ is a $S_{2}$-subgroup of $\mathbb{C}$ and of $\mathfrak{C}_{0}$ and $\Re$ normalizes $\mathfrak{I}_{0}$, since $\mathfrak{I}_{0}=\mathfrak{R} \mathfrak{T} \cap \mathbb{C}$ and $\mathfrak{C} \triangleleft \mathfrak{M}$. Thus, if $\mathfrak{D}_{0}$ is any non identity characteristic subgroup of $\mathfrak{I}_{0}$, then $\mathfrak{S}_{0} \Re \subseteq N\left(\mathfrak{D}_{0}\right)$, so that by Lemma 14.20, $N\left(\mathfrak{D}_{0}\right) \subseteq \mathfrak{M}$. By Theorem 1 of [43], it follows that $\mid \mathfrak{F}_{0}$ : $\sqrt{ } \mid$ is a power of 3 . Since $\mathfrak{E} \subset \mathfrak{C}_{0}, S_{3}$-subgroups of $\mathfrak{F}_{0}$ are not 1 .

Let $\mathfrak{F}_{1}$ be a $S_{2,3}$-subgroup of $\mathfrak{F}_{0}$ which contains $\mathfrak{I}_{0}$, and let $\mathfrak{I}_{1}=$ $\boldsymbol{O}_{2}\left(\mathfrak{C}_{1}\right)$. Thus, $\mathfrak{I}_{1}$ is not characteristic in $\mathfrak{I}_{0}$, so in particular, $\mathfrak{I}_{1} \cap \mathfrak{I}_{0}$. Let $\mathfrak{B}$ be an elementary subgroup of $\mathfrak{M}$ of order 9 , and let

$$
\widehat{\mathfrak{B}}=\left\{B \in \mathfrak{B}^{\sharp} \mid\left[\mathfrak{B}, C_{\mathfrak{B}}(B)\right] \neq 1\right\} .
$$

Since $\mathfrak{B}$ is faithful on $\mathfrak{B}$, we get $\langle\hat{\mathfrak{B}}\rangle=\mathfrak{B}$. If $\hat{\mathfrak{B}}$ normalizes $\mathfrak{I}_{1}$, so does $\mathfrak{B}$, so that $N\left(\mathfrak{I}_{1}\right)$ contains $\mathfrak{S}_{0} \mathfrak{B}$. By Lemma 14.20 , we get $N\left(\mathfrak{I}_{1}\right) \subseteq \mathfrak{M}$, and so $\mathfrak{S}_{1} \subseteq \mathfrak{M}$, whence $\mathfrak{S}_{0} \subseteq \mathfrak{M}$. We may assume that $B$ in $\hat{\mathfrak{B}}$ has been chosen so that $B \notin N\left(\mathfrak{I}_{1}\right)$. Let $\mathbb{L}=\left\langle\mathfrak{F}_{0}, B\right\rangle$. Then $\mathcal{Z}$ normalizes $\mathfrak{B}_{0} \cap C(B) \neq 1$, so $\mathbb{Z}$ is solvable. Since $B \in \hat{\mathfrak{B}}$, Lemma 14.22 implies that $N(\langle B\rangle) \subseteq \mathfrak{M}$. Since $\mathbb{R} \nsubseteq \mathfrak{M}$, Lemma 14.20 implies that $S_{3}$-subgroups of $\mathbb{Z}$ are cyclic. Since $\mathfrak{I}_{1}$ is normalized by a subgroup of $\mathbb{R}$ of order 3 , it follows that $\mathfrak{I}_{1} \subseteq \boldsymbol{O}_{3^{\prime}}(\mathbb{R})$. Hence, $\mathfrak{I}_{1}^{B} \subseteq \boldsymbol{O}_{3^{\prime}}(\mathbb{R})$. Since $\mathfrak{I}_{0}=\left\langle\mathfrak{I}_{1}, \mathfrak{I}_{1}^{B}\right\rangle$, we get $\mathfrak{I}_{0} \subseteq \boldsymbol{O}_{3^{\prime}}(\mathbb{R})$. Hence, $\mathfrak{I}_{0}=\mathfrak{S}_{1} \cap \boldsymbol{O}_{3^{\prime}}(\mathbb{R}) \triangleleft \mathfrak{C}_{1}$. This contradiction shows that this case does not occur.

Case 3. $3 \nmid|\mathfrak{E}|$ and $|\mathfrak{S}|_{2} \neq\left|\mathfrak{S}_{0}\right|_{2}$.

Since $\sqrt{5} \subset \mathfrak{F}_{0}$, we get $|\mathfrak{E}|_{2}<\left|\mathfrak{S}_{0}\right|_{2}$. Again, let $\mathfrak{I}_{0}=\mathfrak{C} \cap \mathfrak{I}$. Since $\Re$ normalizes $\mathfrak{I}_{0}$, we get $\mathfrak{N}\left(\mathfrak{I}_{0}\right) \subseteq \mathfrak{M}$. Since $\mathfrak{I}_{0}$ not a $S_{2}$-subgroup of $\mathfrak{F}_{0}$, we can choose a 2-element $X$ of $N_{\mathfrak{M}}\left(\mathfrak{I}_{0}\right)-\mathfrak{I}_{0}$. Since $X \notin \mathfrak{C}$, we get $\mathfrak{B}_{0}=\mathfrak{夭}_{\mathfrak{s}}(X)$.

Since $\mathfrak{B}$ is 2 -reducible in $\mathfrak{M}$, it follows that $\boldsymbol{O}_{2}\left(\boldsymbol{A}_{\mathfrak{M}}(\mathfrak{B})\right)=1$. Since $\mathfrak{B}_{0}=\boldsymbol{C}_{\mathfrak{B}}(X)$, it follows that $X$ inverts a subgroup $\mathfrak{A}$ of $\boldsymbol{A}_{\mathfrak{M}}(\mathfrak{B})$ of order 3 and that $\mathfrak{B}_{1}=\boldsymbol{C}_{\mathfrak{B}}(\mathfrak{H})$ is of index 4 in $\mathfrak{B}$. Since $3 \nmid|\mathfrak{S}|$, $\mathfrak{M}$ has a subgroup $\mathfrak{X}_{0}$ of order 3 which maps onto $\mathfrak{T}$. Let $\mathfrak{B}$ be an elementary subgroup of $\mathfrak{M}$ of order 9 which contains $\mathfrak{H}_{0}$. Then $\mathfrak{B}$ normalizes $\mathfrak{B}_{1}=\boldsymbol{C}_{\mathfrak{3}}(\mathfrak{Z})$. Hence, $\quad \mathfrak{E}_{0} \subseteq \boldsymbol{C}_{\Theta}\left(\mathfrak{B}_{1}\right) \leqq \boldsymbol{N}_{\Theta}\left(\mathfrak{B}_{1}\right)$. By Lemma 14.20, we get $\boldsymbol{N}_{\Theta}\left(\mathfrak{B}_{1}\right) \subseteq \mathfrak{M}$. The proof is complete. 
Hypothesis 15.1.

(a) $\mathfrak{I}$ is not a $S_{2}$-subgroup of $(3)$.

(b) $|\mathfrak{i}|>8$.

Lemmas 15.2 through 15.15 are proved under Hypothesis 15.1.

LEMMA 15.2. If $1 \subset \mathfrak{D} \triangleleft \mathfrak{I}$ and $|N(\mathfrak{D})|_{2}>|\mathfrak{I}|$, then $\boldsymbol{N}_{\mathfrak{H}}(\mathfrak{D})$ is cyclic.

Proof. The lemma is a consequence of Lemma 14.20 .

LEMMA 15.3.

(a) $\mathfrak{I} / \mathfrak{S}$ has no quaternion subgroup.

(b) $l_{3}(\mathfrak{M})=1$.

Proof. (a) Let $\Re_{0}$ be a subgroup of $\Re$ which is minimal subject to (i) $\mathfrak{S}_{\mathfrak{R}} \triangleleft \mathfrak{T} \mathfrak{R}_{0}$, (ii) $\boldsymbol{C}_{\mathfrak{乏}}\left(\mathfrak{S}_{\mathfrak{C}} \mathfrak{R}_{0} / \mathfrak{K}\right)=\mathfrak{K}_{\mathcal{C}}$. Since $\mathfrak{R} \cap \boldsymbol{O}_{2,3}(\mathfrak{T} \mathfrak{R})$ satisfies (i) and (ii), $\Re_{0}$ exists. By Lemma 5.18 and 0.3.6, it follows that $\Re_{0}$ is of exponent 3 . Since $3 \in \pi_{2}$, we get $m\left(\Re_{0}\right) \leqq 2$. Suppose by way of contradiction that $\mathfrak{I}_{0} / \mathfrak{F}$ is a quaternion subgroup of $\mathfrak{T} / \mathfrak{F}$. In this case, we get $m\left(\Re_{0}\right)=2$, so that $\mathfrak{I} / \mathfrak{S}$ is isomorphic to a subgroup of $G L(2,3)$, and $\mathfrak{S}_{\mathfrak{R}} / \mathfrak{S}_{\mathfrak{C}} \boldsymbol{D}\left(\mathfrak{R}_{0}\right)$ is a chief factor of $\mathfrak{T} \Re_{0}$. Hence,

$$
\boldsymbol{C}_{\Re_{0}}(\boldsymbol{Z}(\mathfrak{T})) \subseteq \boldsymbol{D}\left(\Re_{0}\right)
$$

Let $\mathfrak{A}$ be an abelian subgroup of $\mathfrak{I}$ such that $m(\mathfrak{U}) \geqslant m\left(\mathfrak{U}_{1}\right)$ for every abelian subgroup $\mathfrak{A}_{1}$ of $\mathfrak{I}$ and such that $\mathfrak{A} \nsubseteq \mathfrak{S}$. Notice that $\mathfrak{A}$ exists by Lemma 15.2. Let $\mathfrak{U}_{0}=\mathfrak{2} \cap \mathfrak{F}$. Thus, $\mathfrak{A} / \mathfrak{A}_{0} \cong \mathfrak{U} \mathfrak{F} / \mathfrak{F}$ is either cyclic or is a four-group. Let $\mathfrak{B}_{0}$ be minimal normal subgroup of $\mathfrak{I} \Re_{0}$ which is not centralized by $\Re_{0}$.

Case 1. $\boldsymbol{D}\left(\Re_{0}\right)$ centralizes $\mathfrak{V}_{0}$.

Since $\mathfrak{I}_{0} / \mathfrak{K}$ permutes transitively the subgroups $\mathfrak{R}_{0} \mathfrak{E}_{\mathcal{E}} / \boldsymbol{D}\left(\mathfrak{R}_{0}\right) \mathfrak{S}_{\mathcal{E}}$ of order 3 , it follows that for every subgroup $\Re_{1}$ of $\Re_{0}$ of order $3\left|\boldsymbol{D}\left(\mathfrak{\Re}_{0}\right)\right|,\left|\left[\mathfrak{B}_{0}, \mathfrak{R}_{1}\right]\right| \geqslant 2^{6}$, and $\left|\left[\mathfrak{B}_{0}, \Re_{0}\right]\right| \geqslant 2^{8}$. Since $m\left(\mathfrak{U} / \mathfrak{A}_{0}\right) \leqq 2$, it follows that $\mathfrak{A}$ centralizes a subgroup $\mathfrak{B}_{1}$ of index 4 in $\mathfrak{B}_{0}$, so $\mathfrak{S}\left[\mathfrak{U}, \mathfrak{R}_{0}\right] / \mathfrak{S}$ centralizes a subgroup of $\mathfrak{B}_{0}$ of index $2^{4}$. This is not the case.

Case 2. $\left[\boldsymbol{D}\left(\Re_{0}\right), \mathfrak{B}_{0}\right] \neq 1$, and $\mathfrak{U} \leqq \mathfrak{T}_{0}$.

In this case, $m\left(\mathfrak{X} / \mathfrak{A}_{0}\right)=1$ so that $\mathfrak{A}$ centralizes a hyperplane of $\mathfrak{V}_{0}$. This implies that $\mathfrak{S}\left[\mathfrak{R}_{0}, \mathfrak{N}\right] / \mathfrak{F}$ centralizes a subgroup of $\mathfrak{B}_{0}$ of index 4. This is not the case, since $\mathfrak{S}\left[\Re_{0}, \mathfrak{U}\right]=\mathfrak{S}_{\mathfrak{C}}$. 
Case 3. $\left[\boldsymbol{D}\left(\mathfrak{R}_{0}\right), \mathfrak{B}_{0}\right] \neq 1$ and $\mathfrak{U} \nsubseteq \mathfrak{I}_{0}$.

In this case, $\mathfrak{T} / \mathfrak{F}$ is isomorphic to a $S_{2}$-subgroup of $G L(2,3)$ and $\left|\mathfrak{B}_{0}\right| \geqq 2^{6}$. Since $m\left(\left(\mathfrak{Q} / \mathfrak{U}_{0}\right) \leqq 2\right.$, $\mathfrak{A}$ centralizes a subgrouy of $\mathfrak{B}_{0}$ of index 4. Hence, $\mathfrak{S}_{\mathfrak{c}}\left[\mathfrak{\Re}_{0}, \mathfrak{X}\right] / \mathfrak{S}_{\mathcal{C}}$ centralizes a subgroup of $\mathfrak{B}_{0}$ of index $2^{4}$. This is not the case, since $C_{\mathfrak{B}_{0}}\left(\Re_{0}^{\prime}\right)=1$ and $\mathfrak{R}_{0}^{\prime} \subseteq \mathfrak{S}_{\mathfrak{C}}\left[\Re_{0}, \mathfrak{U}\right]$. Thus, (a) holds.

Since $3 \in \pi_{2}$, (b) is a consequence of (a), since $\mathfrak{T} \mathfrak{R} / \boldsymbol{O}_{2,3}(\mathfrak{T} \mathfrak{R})$ is isomorphic to a subgroup of $G L(2,3)$.

Lemma 15.4. Suppose $X$ is an involution of $\boldsymbol{A}_{\mathfrak{n}}(\mathfrak{B}),\left|\mathfrak{B}: \boldsymbol{C}_{\mathfrak{B}}(X)\right|=2$ and $\boldsymbol{C}_{\Theta}([\mathfrak{B}, X]) \nsubseteq \mathfrak{M}$. Then $\mathfrak{R}$ is abelian of type $\left(3,3^{a}\right)$ for some $a \geqq 1$ and $S_{3}$-subgroups of $\boldsymbol{C}_{\mathfrak{n}}([\mathfrak{S}, X])$ are cyclic of order $3^{a}$.

Proof. Let $T$ be a 2-element of $\mathfrak{M}$ which maps to $X$. We assume without loss of generality that $T \in \mathfrak{T}$. This assumption is justified since it suffices to prove the lemma for some $\mathfrak{M}$-conjugate of $\boldsymbol{C}_{\mathfrak{F}}(X)$.

Since $X$ centralizes a hyperplane of $\mathfrak{B}, X$ centralizes $\boldsymbol{O}_{3^{\prime}}\left(\boldsymbol{A}_{\mathfrak{n}}(\mathfrak{B})\right)$. Hence, $T \notin \boldsymbol{O}_{3},(\mathfrak{M})$, so $T \notin \mathfrak{S}$. Let $\mathfrak{L}=\mathfrak{S} \mathfrak{R}\langle T\rangle, \mathfrak{R}=\boldsymbol{A}_{\mathfrak{\varepsilon}}(\mathfrak{B})$. By Lemma 15.3(b), $l_{3}(\Re)=1$ (since 3||$\left.\Re \mid\right)$. Let $\Omega_{0}$ be the normal closure of $X$ in R. Since $X$ centralizes $\boldsymbol{O}_{3^{\prime}}\left(\boldsymbol{A}_{\mathfrak{n}}(\mathfrak{B})\right)$, so does $\Re_{0}$. Since $\boldsymbol{O}_{2}\left(\boldsymbol{A}_{\mathfrak{n}}(\mathfrak{B})\right)=1$ and $3 \in \pi_{2}$, it follows that $\Re_{0} \cong \Sigma_{3}$. Hence, $\Re=\Re_{0} \times \Re_{1}$, where $\Re_{1}=C_{\Omega}\left(\Re_{0}\right)$. This implies that $\Re_{1}$ centralizes the four-group [, $\Re_{0}$. Let $\mathscr{\Omega}_{1}$ be the preimage of $\Re_{1}$ in $\mathbb{R}$. Thus, $\mathfrak{S}_{1} \triangleleft \mathbb{Q}$ and $\mathfrak{R} / \mathfrak{R}_{1} \cong \Re_{0} \cong \Sigma_{3}$. Hence, $\mathfrak{S}_{1} \cap \Re$ is of index 3 in $\Re$. Since $[\mathfrak{B}, X] \subset\left[\mathfrak{B}, \Re_{0}\right] \subseteq C\left(\Re_{1} \cap \Re\right)$, and since by hypothesis, $C_{\circledast}([\mathfrak{B}, X]) \nsubseteq \mathfrak{M}$, Lemma 14.20 implies that $\mathfrak{\mho}_{1} \cap \Re$ is cyclic of order $3^{a}$ for some $a \geqslant 1$, Since $\left|\Re: \Omega_{1} \cap \Re\right|=3$, $\Re$ is metacyclic. By $0.3 .8, \mathfrak{R}^{\prime}=1$. The proof is complete.

Let $\mathfrak{W}=\boldsymbol{V}\left(\operatorname{ccl}_{\mathbb{S}}(\mathfrak{B}) ; \mathfrak{I}\right)$.

LEMMA 15.5. $\mathfrak{W} \Phi \mathfrak{c}$, where $\mathfrak{c}=\boldsymbol{C}(\mathfrak{B})$.

Proof. Suppose $\mathfrak{W} \leqq \mathbb{E}$. Let $\mathfrak{I}_{0}=\mathfrak{I} \cap \mathfrak{E}$ so that $\mathfrak{W} \cong \mathfrak{I}_{0}$. Since $\mathfrak{W}$ is weakly closed in $\mathfrak{I}_{0}$, we get $N_{\Re}\left(\mathfrak{I}_{0}\right) \subseteq N_{\Re}(\mathfrak{W})$. By Lemma 15.2, $\boldsymbol{N}_{\Re}(\mathfrak{W})=\Re_{1}$ is cyclic.

Since $\Omega_{1}(Z(\mathfrak{I})) \subseteq \mathfrak{S}$, Lemma 15.2 implies that $\Re \cap \mathfrak{S}=\Re_{0}$ is cyclic. Since $\Re=\Re_{0} \Re_{1}$ and $\Re_{0} \triangleleft \Re$, it follows that $\Re$ is metacyclic, so by 0.3.8, $\Re^{\prime}=1$. Since $3 \in \pi_{2}, \Re_{i} \neq 1, i=1,2$. Since $\Omega_{1}(\Re)=\left(\Omega_{1}(\Re) \cap \mathfrak{V}\right)$. $\left(\Omega_{1}(\Re) \cap N\left(\mathfrak{I}_{0}\right)\right)$, it follows that $\Re_{0} \cap \Re_{1}=1$. Hence, $\Re=\Re_{0} \times \Re_{1}$.

Let $\mathfrak{I}_{1}=\boldsymbol{O}_{2}\left(\mathfrak{I}_{0} \mathfrak{R}_{0}\right)$. Thus, $\mathfrak{I}_{1} \triangleleft \mathfrak{I} \Re$. Since $\mathfrak{C} \triangleleft \mathfrak{M}$, we also have $\mathfrak{I}_{0} \mathfrak{R}_{0} \triangleleft \mathfrak{T} \Re$, as $\mathfrak{I}_{0} \mathfrak{R}_{0}=\mathfrak{C} \cap \mathfrak{T} \Re$. Hence, $\mathfrak{T} \mathfrak{R}_{0}=\mathfrak{Q}$ is a group. Let $\mathfrak{R}_{1}=$ $\boldsymbol{O}_{2}(\mathfrak{L}) \supseteq \mathfrak{I}_{1}$. Since $\mathfrak{R}_{1} / \mathfrak{I}_{1}$ is represented faithfully on $\mathfrak{S} \mathfrak{R} / \mathfrak{S}_{\mathfrak{C}} \mathfrak{R}_{0}$, we get $\left|\mathfrak{Z}_{1}: \mathfrak{I}_{1}\right| \leqq 2$. We argue that $V\left(\operatorname{ccl}_{\mathscr{\Theta}}(\mathfrak{B}) ; \mathfrak{R}_{1}\right)=V\left(\operatorname{ccl}_{\Theta}(\mathfrak{B}) ; \mathfrak{I}_{1}\right)$. Since $\mathfrak{L}_{1} \supseteqq \mathfrak{I}_{1}$, we have $V\left(\operatorname{cel}_{\mathscr{S}}(\mathfrak{B}) ; \mathfrak{R}_{1}\right) \supseteqq V\left(\operatorname{cl}_{\mathscr{\Theta}}(\mathfrak{B}): \mathfrak{I}_{1}\right)$. Suppose $G \in \mathfrak{S S}^{3}$ and $\mathfrak{B}^{(*} \cong \mathfrak{L}_{1}$. 
Since $\mathfrak{R}_{1} \subseteq \mathfrak{I}$, we have $\mathfrak{B}^{G} \subseteq \mathfrak{W} \subseteq \mathfrak{C}$. Since $\mathfrak{I}_{1}=\mathfrak{Q}_{1} \cap \mathfrak{F}$, we get $\mathfrak{B}^{G} \subseteq \mathfrak{I}_{1}$.

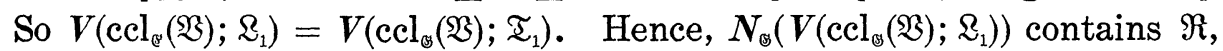
so by Lemma $14.20, N_{\mathscr{G}}\left(V\left(\operatorname{ccl}_{\mathscr{G}}(\mathfrak{B}) ; \mathfrak{R}_{1}\right)\right) \subset \mathfrak{M}$.

Let $\mathbb{B}_{2}$ be a $S_{2}$-subgroup of (S) which contains $\mathfrak{T}$. Since $Z\left(\mathbb{S}_{2}\right) \subseteq N\left(\mathfrak{S}_{2}\right)$, Lemma 14.19 implies that $\boldsymbol{Z}\left(\mathbb{S}_{2}\right) \leqq \mathfrak{T}$. Hence, $\boldsymbol{Z}\left(\mathbb{S}_{2}\right) \subseteq \boldsymbol{Z}(\mathfrak{T})$. Since $\Omega_{1}(Z(\mathfrak{I})) \leqq \mathfrak{B}$, it follows that we can choose $Z$ in $\boldsymbol{Z}\left(\mathfrak{S}_{2}\right)^{\sharp} \cap \mathfrak{B}$. Let $\mathfrak{S}=\boldsymbol{C}(Z) \supseteqq \mathfrak{T} \Re_{0}$.

By Lemma 14.22, $N\left(\Omega_{1}\left(\Re_{0}\right)\right) \subseteq \mathfrak{M}$. By Lemma 14.20 , it follows that $S_{3}$-subgroups of $\subseteq$ are cyclic, since $\subseteq \nsubseteq \mathfrak{M}$. Hence, $\mathfrak{R}_{1} \subseteq \boldsymbol{O}_{3^{\prime}}(\mathfrak{S})$, since $\mathfrak{R}_{0} \subseteq \mathfrak{S}$. Let $\mathfrak{S}_{0}$ be a $S_{2,3}$-subgroup of $\mathfrak{S}$ which contains $\mathfrak{T} \Re_{0}$, and let $\left\{\mathfrak{S}_{2}, \mathfrak{S}_{3}\right\}$ be a Sylow system of $\mathfrak{S}_{0}$ with $\mathfrak{I} \subseteq \mathfrak{S}_{2}, \Re_{0} \subseteq \mathfrak{S}_{3}$. Thus, $\mathfrak{S}_{3}$ is cyclic and $\mathfrak{S}_{3} \subseteq \mathfrak{M}$, by Lemma 14.22. Let $\mathfrak{S}_{1}=\boldsymbol{O}_{2}\left(\mathfrak{S}_{0}\right)$, so that $\mathfrak{S}_{1} \supseteqq \Omega_{1}$. Since $\mathfrak{S}_{3}$ is cyclic, we get $\widetilde{S}_{0}=\mathfrak{S}_{1} N_{\widetilde{\Xi}_{0}}\left(\Re_{0}\right)$. Since $\mathfrak{S}_{3} \nsubseteq \mathfrak{M}$, and $N\left(\Re_{0}\right) \subseteq \mathfrak{M}$, we get $\widetilde{S}_{1} \nsubseteq \mathfrak{M}$. Hence, $\widetilde{S}_{1} \supset \mathbb{R}_{1}$.

Since $\left(\mathfrak{S}_{1} \cap \mathfrak{M}\right) \mathfrak{I}$ is a 2-subgroup of $\mathfrak{M}$, we get $\mathfrak{S}_{1} \cap \mathfrak{M} \subseteq \mathfrak{I}$. Hence, $\mathfrak{S}_{1} \cap \mathfrak{M}=\mathfrak{\Omega}_{1}$. Since $N\left(V\left(\operatorname{ccl}_{\mathfrak{G}}(\mathfrak{B}) ; \mathbb{R}_{1}\right) \subseteq \mathfrak{M}\right.$, we conclude that $N_{\mathbb{\Xi}_{1}}\left(\mathbb{R}_{1}\right) \subseteq \mathfrak{M}$. This contradiction completes the proof.

\section{LEMMA 15.6. $\Re \cong \mathfrak{M}^{\prime}$.}

Proof. By Lemma 14.10, we may assume that $\mathfrak{R}^{\prime}=1$. Since $3 \in \pi_{2}, \quad A_{\circledast}(\Re)$ is a 2-group. Since $\Re \cong N(\Re)^{\prime}$, it follows that $N(\Re)$ contains an element $X$ which inverts $\Re$. Since $X$ normalizes every subgroup of $\mathfrak{R}$, Lemma 14.22 implies that $X \in \mathfrak{M}$. The proof is complete.

Lemma 15.7. Suppose $X$ is an involution of $\mathfrak{I}$ and $\left|\mathfrak{B}: C_{\mathfrak{B}}(X)\right|=2$. Then $\mathfrak{B} \subseteq \boldsymbol{O}_{2}(\boldsymbol{C}([\mathfrak{B}, X]))$.

Proof. Let $\mathfrak{X}=\boldsymbol{C}(\mathfrak{Y})$, where $\mathfrak{Y}=[\mathfrak{B}, X]$. Since $\left|\mathfrak{B}: \boldsymbol{C}_{\mathfrak{B}}(X)\right|=2$, we get $|\mathfrak{Y}|=2$. Let $\mathfrak{A}$ be a subgroup of $O_{3}(\mathfrak{M} / \mathfrak{S})$ which is of order 3 and is inverted by $X$. Then $[\mathfrak{B}, \mathfrak{X}]=4$, and $[\mathfrak{B}, \mathfrak{2}] \supset \mathfrak{Y}$. Since $3 \in \pi_{2}$

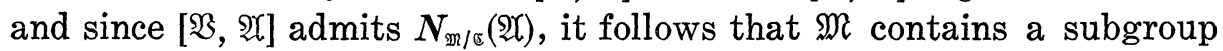
$\mathfrak{B}$ of order 3 which centralizes [ỉ, $\mathfrak{i}]$. By Lemma 14.22, we have $N(\mathfrak{B}) \subseteq \mathfrak{M}$.

We assume without loss of generality that $\mathfrak{X} \nsubseteq \mathfrak{M}$. By Lemma $14.20, S_{3}$-subgroups of $\mathfrak{X} \cap \mathfrak{M}$ are cyclic. Since $\mathfrak{B} \subseteq \mathfrak{X}$, and $N(\mathfrak{B}) \subseteq \mathfrak{M}$, $S_{3}$-subgroups of $\mathfrak{X}$ are cyclic. By Lemma $15.4, \mathfrak{R}^{\prime}=1$ and $\mathfrak{R}$ is of type $\left(3,3^{a}\right)$. By Lemma $15.6, \mathfrak{R} \subseteq \mathfrak{M}^{\prime}$.

Since $\mathfrak{R} \subseteq \mathfrak{M}^{\prime}, \quad N_{\mathfrak{x}}(\Re)$ contains an element $I$ which inverts $\Re$.

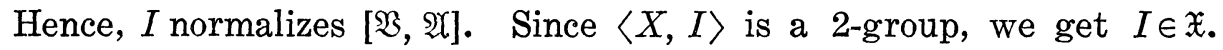
Hence, $\mathfrak{B} \subseteq \mathfrak{X}^{\prime}$.

Since $\sigma_{2}=\{3\}, \mathfrak{B} \subseteq \mathfrak{X}^{\prime}$ and $N(\mathfrak{B}) \subseteq \mathfrak{M}$, it follows that $\mathfrak{M} \cap \mathfrak{X}$ contains a $S_{2}$-subgroup $\mathfrak{D}$ of $\mathfrak{X}$. Since $\mathfrak{B} \in \boldsymbol{U}_{\mathfrak{x}}(\mathfrak{D} ; 2)$, we get $\mathfrak{B} \subseteq \boldsymbol{O}_{2}(\mathfrak{X})$, 
as required.

LEMMA 15.8. If $\mathfrak{B}_{0}$ is a hyperplane of $\mathfrak{B}$, then $\left|\boldsymbol{C}_{\mathfrak{G}}\left(\mathfrak{R}_{0}\right): \mathbb{S}\right| \leqq 2$.

Proof. By Lemma 15.3, $C\left(\mathfrak{B}_{0}\right) \subseteq \mathfrak{M}$. Since $C\left(\mathfrak{B}_{0}\right) / \mathfrak{C}$ stabilizes $\mathfrak{B} \supset \mathfrak{B}_{0} \supset 1$, it follows that $\boldsymbol{C}\left(\mathfrak{B}_{0}\right) / \mathfrak{S}$ is an elementary 2-group. Since $\boldsymbol{C}\left(\mathfrak{B}_{0}\right) / \mathfrak{E}$ is represented faithfully on $\boldsymbol{O}_{2}{ }^{\prime}(\mathfrak{M} / \mathfrak{E})$, the lemma follows.

Lemma 15.9. Suppose $\mathfrak{V}_{0}$ is of index 4 in $\mathfrak{B}$. Then $\boldsymbol{C}_{\mathfrak{M}}\left(\mathfrak{V}_{0}\right)$ does not contain a four-group (5) such that

(a) $\quad 5 \cap \mathbb{E}=1$.

(b) $|[\mathfrak{B}, \mathfrak{s}]|=2$.

Proof. Suppose false. By Lemma 5.34, M/C contains a subgroup $\left(\mathfrak{S}=\mathfrak{D}_{1} \times \mathfrak{D}_{2}\right.$ such that $\left|\mathfrak{D}_{i}\right|=p_{i}$ is of odd prime order, $\mathfrak{D}_{i}$ admits $\mathfrak{F}_{i}, i=1,2$, and $C_{\mathbb{E}}(\mathfrak{D})=1$.

Let $\left.\left.\mathfrak{B}_{0}=\right] \mathfrak{B}, \mathfrak{D}\right], \mathfrak{B}_{1}=C_{\mathfrak{B}_{0}}(\mathfrak{F})$. Thus, $\left|\mathfrak{B}_{0}: \mathfrak{B}_{1}\right| \leqq 4$ and $\left|\left[\mathfrak{B}_{0}, \mathfrak{E}\right]\right|=2$. Since $\mathfrak{D}$ is represented faithfully on $\mathfrak{B}_{0}$, the inequality is an equality. On the other hand, $\left[5\right.$ contains a subgroup $\mathfrak{F}_{0}=\langle E\rangle$ of order 2 such that $E$ inverts $\mathfrak{D}$. Hence, $\mathfrak{B}_{0}$ is a free $F_{2} \mathfrak{F}_{0}$-module, so if $\left|\mathfrak{B}_{0}\right|=2^{2 w}$, that $\left|\left[\mathfrak{B}_{0}, \mathfrak{F}_{0}\right]\right|=2^{w}$. This implies that $w \leqq 1$, since $\left[\mathfrak{B}_{0}, \mathfrak{F}_{0}\right] \subseteq[\mathfrak{B}, \mathfrak{F}]$, and $\mid[\mathfrak{B},[\mathbb{F}] \mid=2$. This is absurd, since $\mathfrak{D}$ is represented faithfully on $\mathfrak{B}_{0}$. The proof is complete.

LEMMA 15.10. If $G \in \mathbb{S}$ and $\mathfrak{B}^{a} \leqq \mathfrak{I}$, then $\left|\mathfrak{B}^{G}: \mathfrak{B}^{G} \cap \mathfrak{S}\right| \leqq 2$.

Proof. Let $\mathfrak{B}^{*}=\mathfrak{B}^{G}, \mathfrak{B}_{0}^{*}=\mathfrak{B}^{*} \cap \mathfrak{E}$, and let $2^{a}=\left|\mathfrak{B}^{*} ; \mathfrak{B}_{0}^{*}\right|$. Suppose by way of contradiction that $\alpha \geqslant 2$. By Lemma 5.34, M/ a subgroup $\mathfrak{D}=\mathfrak{D}_{1} \times \cdots \times \mathfrak{D}_{a}$ such that $\left|\mathfrak{D}_{i}\right|=p_{i}$ is an odd prime, $\mathfrak{D}_{i}$ admits $\mathfrak{S}^{*}, 1 \leqq i \leqq a$, and such that $\boldsymbol{O}_{\mathfrak{B}}(\mathfrak{D}) \mathfrak{S}_{0}^{*}$.

Let $\mathfrak{X}=[\mathfrak{B}, \mathfrak{D}], \mathfrak{X}_{i}=\boldsymbol{C}_{\mathfrak{X}}\left(\mathfrak{D}_{i}\right)$. We argue that $\mathfrak{X}_{i}=1,1 \leqq i \leqq \alpha$. Suppose false. Since $\mathfrak{X}_{i}$ admits $\mathfrak{D}$ and $\mathfrak{B}^{*}$, and since $\boldsymbol{C}_{\mathfrak{x}}(\mathfrak{D})=1$, we can choose $j$ such that $\left[\mathfrak{X}_{i}, \mathfrak{D}_{j}\right] \neq 1$. Let $\mathfrak{D}_{j}=\mathfrak{E}_{j} / \mathfrak{D}$, where $\mathfrak{E}_{j} \supset \mathfrak{E}$, and let $\mathfrak{Y}_{i j}$ be a subgroup of $\left[\mathfrak{X}_{i}, \mathfrak{D}_{j}\right]$ which is minimal subject to admitting $\mathbb{C}_{j} \mathfrak{B}^{*}$. Let $\mathfrak{B}_{j}^{*}=\boldsymbol{C}_{\mathfrak{B}^{*}}\left(\mathfrak{D}_{j}\right)$, so that $\left|\mathfrak{B}^{*}: \mathfrak{B}_{j}^{*}\right|=2$. By minimality of $\mathfrak{Y}_{i j}$, we get $\left.\mathfrak{B}_{j}^{*}, \mathfrak{Y}_{i j}\right]=1$. By Lemma 15.1, we have $\mathfrak{Y}_{i j} \leqq \mathfrak{M}^{G}$, and by Lemma 15.8, $\left|\mathfrak{Y}_{i j}: \mathfrak{Y}_{i j} \cap \mathfrak{S}^{G}\right| \leqq 2$. Hence, $\left|\mathfrak{D}_{j}\right|=3$, and $\left|\mathfrak{Y}_{i j}\right|=4$. Choose $\mathfrak{Y} \in \mathfrak{Y}_{i j}-\mathfrak{Y}_{i j} \cap \mathfrak{S}^{G}$, and let $3=\left[Y, \mathfrak{B}^{*}\right]=\langle Z\rangle$. By Lemma 15.7, $\mathfrak{B}^{G} \subseteq \boldsymbol{O}_{2}(\boldsymbol{C}(Z))$. On the other hand, $\mathfrak{D}_{i}=\mathfrak{S}_{i} / \mathfrak{S}$ and $\mathfrak{S}_{i}$ centralizes $Z$. Hence, $\left[\mathfrak{C}_{i}, \mathfrak{B}^{*}\right]$ is a 2-group, against $C_{\mathfrak{D}}\left(\mathfrak{B}^{*}\right)=1$. Hence, $\mathfrak{X}_{i}=1$, $1 \leqq i \leqq a$.

By Lemma 5.47, $\mathfrak{X}$ is a free $F_{2} \mathfrak{B}^{*} / \mathfrak{B}_{0}^{*}$-module. Let $\mathfrak{X}=\mathfrak{X}_{1} \times \cdots \times \mathfrak{X}_{a}$, where each $\mathfrak{X}_{i}$ is isomorphic to $F_{2} \mathfrak{V}^{*} / \mathfrak{V}_{0}^{*}$ as $\mathfrak{B}^{*}$-module. Since $a \geqslant 2$, $\mathfrak{X}_{1}$ contains a subgroup $\mathfrak{Y}$ of order 8 which admits $\mathfrak{B}^{*}$. Since $\left|C_{\mathfrak{x}_{1}}\left(\mathfrak{B}^{*}\right)\right|=2$, 
it follows that $\left|\boldsymbol{C}_{\mathfrak{y}}\left(\mathfrak{B}^{*}\right)\right|=2$. Hence, $\left[\mathfrak{Y}, \mathfrak{B}^{*}\right]=\boldsymbol{C}_{\mathfrak{y}}\left(\mathfrak{B}^{*}\right)$. Since $\mathfrak{Y}=$ $\left\langle\boldsymbol{C}_{\mathfrak{g}}(\tilde{\mathfrak{B}})\right\rangle$, where $\tilde{\mathfrak{B}}$ range over all the hyperplanes of $\mathfrak{B}^{*}$, Lemma 15.1 implies that $\mathfrak{Y} \subseteq \mathfrak{M}^{G}$. This contradicts Lemma 15.9. The proof is complete.

The next lemma is delicate and elaborate.

LEMMA 15.11. $|\mathfrak{B}: \mathfrak{W} \cap \mathfrak{C}| \geqslant 4$, where $\mathfrak{W}=V\left(\operatorname{ccl}_{\mathfrak{S}}(\mathfrak{B})\right.$; $\left.\mathfrak{T}\right)$.

Proof. Suppose false. By Lemma 15.5, we get $|\mathfrak{W}: \mathfrak{W} \cap \mathfrak{C}|=2$. Let $\mathfrak{W}_{0}=\mathfrak{W} \cap \mathbb{E}$. Let $\mathfrak{I}_{1}=\mathfrak{I} \cap \mathfrak{E}, \mathfrak{I}_{0}=\mathfrak{I}_{1} \mathfrak{W}$. Thus, $\mathfrak{I}_{i} \triangleleft \mathfrak{I}, i=0,1$, and $\left|\mathfrak{I}_{0}: \mathfrak{I}_{1}\right|=2$.

Since $\left|\mathfrak{M}: \mathfrak{F}_{0}\right|=2$, there is $G$ in $\mathbb{B}$ such that $\mathfrak{B}^{*}=\mathfrak{B}^{a} \subseteq \mathfrak{I}$, and $\mathfrak{B}^{*} \nsubseteq \mathfrak{C}$. Hence, $\mathfrak{W}=\mathfrak{W}_{0} \mathfrak{B}^{*}, \mathfrak{I}_{0}=\mathfrak{I}_{1} \mathfrak{B}^{*}$. Let $\mathfrak{B}_{0}^{*}=\mathfrak{B}^{*} \cap \mathfrak{E}$, so that $\mathfrak{B}_{0}^{*}$ is a hyperplane of $\mathfrak{B}^{*}$. By Lemma 15.8 applied to $\mathfrak{M}^{G}$, we have $\left|\mathfrak{B}: C_{\mathfrak{B}}\left(\mathfrak{B}^{*}\right)\right|=2$, and by Lemma 15.1, $\mathfrak{B} \subseteq \mathfrak{M}^{a}$.

Let $3=\langle Z\rangle=\left[\mathfrak{B}, \mathfrak{B} *\right.$, so that $|3|=2,3 \subseteq \mathfrak{B} \cap \mathfrak{B}^{*}$. Since $\mathfrak{W} \triangleleft \mathfrak{I}$, we get $\mathfrak{W} \mathbb{C} / C \triangleleft \mathfrak{I} / \mathfrak{C}$. Thus, $\mathfrak{W} \mathfrak{C} / \mathfrak{C} \cong \mathfrak{W} / \mathfrak{W}_{0}$ is a central subgroup of $\mathfrak{I} / \mathfrak{S}$ of order 2. Let $\mathfrak{D}=\left[\boldsymbol{O}_{2^{\prime}}(\mathfrak{M} / \mathfrak{C}), \mathfrak{W}\right]$. Thus, $\mathfrak{D}$ admits $\mathfrak{T}$. Since $\left|\mathfrak{B}: C_{\mathfrak{B}}\left(\mathfrak{B}^{*}\right)\right|=2$, it follows that $|\mathfrak{D}|=3$ and that $\mathfrak{B}=\mathfrak{B}^{0} \times \mathfrak{B}^{1}$, where $\mathfrak{B}^{0}=\boldsymbol{C}_{\mathfrak{B}}(\mathfrak{D}), \mathfrak{B}^{1}=[\mathfrak{B}, \mathfrak{D}]$, and $\mathfrak{B}^{1}$ is a four-group containing 3. More explicitly, both $\mathfrak{B}^{0}$ andỉ $\mathfrak{B}^{1}$ admit $\mathfrak{I}$ and $\left[\mathfrak{W}, \mathfrak{B}^{1}\right]=\mathfrak{3}=\left[\mathfrak{B}^{*}, \mathfrak{B}^{1}\right]=\left[\mathfrak{B}^{*}, \mathfrak{B}\right]$. Since $|\mathfrak{B}|>8$, it follows that $\mathfrak{B}^{0} \neq 1$. Hence, $Z(\mathfrak{I})$ is non cyclic since $\Omega_{1}(\boldsymbol{Z}(\mathfrak{I}))=\left(\Omega_{1}(\boldsymbol{Z}(\mathfrak{I})) \cap \mathfrak{B}^{0}\right) \times 3$.

Let $\mathfrak{D}=\mathbb{F} / \mathbb{C}$ and let $\mathbb{F}_{3}$ be a $S_{3}$-subgroup of $\mathbb{F}$ with $\mathfrak{F}_{3} \subseteq \Re$. Since $\mathbb{F} \triangleleft \triangleleft \mathfrak{M}, \mathfrak{F}_{3}$ exists. By construction, $\mathfrak{F}_{3}$ covers $\mathfrak{D}$. Since $\mathfrak{B}^{1}=\left[\mathfrak{B}, \mathfrak{F}_{3}\right]$, it follows that $\mathfrak{B}^{1}$ admits $N_{\Re}\left(\mathfrak{F}_{3}\right)$, a non cyclic group. Since $\left|\mathfrak{B}^{1}\right|=4$, we can choose a subgroup $\mathfrak{A}$ of $N_{\mathfrak{R}}\left(\breve{צ}_{3}\right)$ of order 3 such that $\left[\mathfrak{V}^{1}, \mathfrak{X}\right]=1$.

We next show that $N(\mathfrak{U}) \subseteq \mathfrak{M}$. Namely, let $\mathfrak{B}$ be a subgroup of $N_{\mathfrak{R}}\left(\mathfrak{F}_{3}\right)$ which is elementary of order 9 and contains $\mathfrak{A}$. If $\mathfrak{B}$ centralizes $\mathfrak{B}^{1}$, then $\mathfrak{B} \in \mathscr{C}^{*}(\mathfrak{S})$, by Lemma 14.21. If $\mathfrak{B}$ does not centralize $\mathfrak{B}^{1}$, then $N(\mathfrak{U}) \subseteq \mathfrak{M}$, by Lemma 14.22. Thus, in both cases, $N(\mathfrak{N}) \subseteq \mathfrak{M}$.

We have succeeded in showing that $\boldsymbol{C}_{\mathfrak{n}}([\mathfrak{B}, \mathfrak{B} *])$ contains a subgroup $\mathfrak{A}$ of order 3 such that $N(\mathfrak{N}) \subseteq \mathfrak{M}$. By symmetry, $\boldsymbol{C}_{\mathfrak{M}} G\left(\left[\mathfrak{B}, \mathfrak{B}^{*}\right]\right)$ contains a subgroup $\mathfrak{2}^{*}$ of order 3 such that $N\left(\mathfrak{N}^{*}\right) \subseteq \mathfrak{M}^{G}$. This symmetry exists, since if $\mathfrak{T}^{*}$ is a $S_{2}$-subgroup of $\mathfrak{M}^{G}$ which contains $\mathfrak{B}$ and $\tilde{\mathfrak{S}}=V\left(\operatorname{ccl}_{\mathfrak{G}}(\mathfrak{B}) ; \mathfrak{I}^{*}\right)$, then $\left|\tilde{\mathfrak{B}}: \tilde{\mathfrak{B}} \cap \boldsymbol{C}\left(\mathfrak{B}^{*}\right)\right|=2$.

Suppose by way of contradiction that $C(\mathbb{3}) \subseteq \mathfrak{M}$. Then $\mathfrak{2}^{*} \subseteq \mathfrak{M}$, so $S_{3}$-subgroups of $\mathfrak{M} \cap \mathfrak{M}^{G}$ are non cyclic. Since $\boldsymbol{O}_{2}\left(\mathfrak{M}^{G}\right) \subseteq C(3)$, it follows from Lemma 14.20 applied to $\mathfrak{M}^{G}$ that $\mathfrak{M}=\mathfrak{M}^{a}$. However, $\mathfrak{B}^{*} \nsubseteq \mathfrak{C}$, while $\mathfrak{B} \subseteq \mathbb{C}$. We conclude that $\boldsymbol{C}(\mathfrak{3}) \nsubseteq \mathfrak{M}$. In particular, $S_{3}$-subgroups of $C(3)$ are cyclic, by Lemma 14.20 .

We next show that $\mathfrak{D} \triangleleft \mathfrak{M} / \mathfrak{C}$. Since $\mathfrak{D}$ admits $\mathfrak{I}$ and since $\mathfrak{D} \subseteq \boldsymbol{F}(\mathfrak{M} / \mathfrak{C})$, it follows that $\mathfrak{D} \triangleleft \mathfrak{M} / \mathfrak{C}$ if and only if $\mathfrak{D} \triangleleft \mathfrak{S} / \mathfrak{S}$. Here we are using $3 \in \pi_{2}$ to conclude that $\boldsymbol{A}_{\mathbb{S}}(\mathfrak{X})$ is a 2, 3-group for every 
section $\mathfrak{X}$ of $\mathbb{B}$ which is a 3 -group. Since $[\mathfrak{D}, \mathfrak{B}]$ is of 4 , it follows that $\mathfrak{D}$ is normal in $\mathfrak{R} \mathbb{S} / \mathbb{E}$.

Since $\mathfrak{D} \triangleleft \mathfrak{M} / \mathfrak{C}$, it follows that $\mathfrak{B}^{1} \triangleleft \mathfrak{M}$. Since $S_{3}$-subgroups of $\boldsymbol{C}_{\mathfrak{m}}(3)$ are cyclic, it follows that $\boldsymbol{C}_{\mathfrak{\Re}}\left(\mathfrak{B}^{1}\right)$ is cyclic and of index 3 in $\Re$. Thus, $\Re$ is metacyclic, so by $0.3 .8, \Re^{\prime}=1$.

We next show that $\mathfrak{I} / \mathfrak{S}$ is elementary of order 2 or 4 . Namely, $\mathfrak{R}^{\prime}=1, \mathfrak{S} \mathfrak{R} \triangleleft \mathfrak{T} \mathfrak{R}$, and $\mathfrak{I} / \mathfrak{S}$ is represented faithfully on $\mathfrak{R} \mathfrak{S} / \mathfrak{S}$. Since $\mathfrak{B}^{*} \nsubseteq \boldsymbol{O}_{3^{\prime}}(\mathfrak{M})$, we get $\mathfrak{S} \subset \mathfrak{T}$.

Since $\mathfrak{B} \subseteq \boldsymbol{Z}\left(\mathfrak{S}_{0}\right)$, we may view $\mathfrak{B}$ as a $\mathfrak{T} \mathfrak{R} / \mathfrak{S}_{0}$-group. Let $\mathfrak{R}_{0}=$ $\Omega_{1}(\Re)$, so that $\mathfrak{I} / \mathfrak{F}_{\mathcal{C}}$ is represented faithfully on $\mathfrak{S}_{\mathfrak{Z}} \Re_{0} / \mathscr{K}_{\mathcal{C}}$. Since $\mathfrak{R}_{0}$ centralizes a $S_{2^{\prime}}$-subgroup of $\boldsymbol{O}_{3^{\prime}}(\mathfrak{M})$, it follows that $\left[\mathfrak{H}_{2}, \Re_{0}\right] \subseteq \mathfrak{S}_{0}$. Hence, $\mathfrak{S}_{0} \Re_{0} \triangleleft \mathfrak{I} \Re$. To obtain our assertion that $\mathfrak{I} / \mathfrak{S}$ is elementary of order 2 or 4 , it suffices to show that $\mathfrak{S}_{0} \Re_{0} / \mathscr{S}_{0}$ contains a subgroup of order 3 which admits $\mathfrak{T}$. If $\Re_{0} \cap \mathfrak{C} \neq 1$, then $\left|\Re_{0} \cap \mathfrak{E}\right|=3$ and $\mathfrak{S}_{0}\left(\Re_{0} \cap \mathfrak{E}\right) / \mathfrak{S}_{0}$ admits $\mathfrak{T}$. If $\mathfrak{R}_{0} \cap \mathfrak{F}=1$, then $\mathbb{E}$ is a $3^{\prime}$-group; and since $\mathfrak{D} \triangleleft \mathfrak{M} / \mathbb{C}$, we get that $\left|\Re_{0} \cap \mathfrak{F}\right|=3$. We conclude that $\mathfrak{T} / \mathfrak{F}$ is elementary of order $2^{a}$, where $a=1$ or 2 .

Choose $X$ in $Z(\mathfrak{T})^{\sharp} \cap \mathfrak{B}^{0}$. We will show that $\boldsymbol{C}(X) \cong \mathfrak{M}$. Suppose false. Let $\Omega_{0}=C_{\mathfrak{n}}(X), \Re=\boldsymbol{C}(X), \mathfrak{D}=\mathbb{F} / \mathbb{C}$ and let $\widetilde{\mho}_{3}$ be a $S_{3}$-subgroup of $\Re_{0}$ which contains a $S_{3}$-subgroup $\mathfrak{F}_{3}$ of $\mathfrak{D}$. Since $C(X) \nsubseteq \mathfrak{M}, \widetilde{\mho}_{3}$ is cyclic. We assume without loss of generality that $V^{*}$ in $\mathfrak{B}^{*}$ inverts $\widetilde{\mho}_{3}$. This assumption justified since each element of $\mathfrak{B}^{*}-\mathfrak{B}_{0}^{*}$ inverts $D$ and $|\mathfrak{D}|=3$.

Let $E$ be an element of $\mathfrak{F}_{3}$ of order 3 and let $\mathfrak{B}$ be an elementary subgroup of $\mathfrak{M}$ of order 9 which contains $E$. Then $\mathfrak{B} \nsubseteq \mathfrak{F}_{3}$, so $\mathfrak{B}$ does not centralize $X$, so $N(\langle E\rangle) \subseteq \mathfrak{M}$, by Lemma 14.22 . Since $\mathfrak{B}^{*}$ inverts $E$ and centralizes $X$, we get $E \in \Re^{\prime}$. Since $\sigma_{2}=\{3\}$, it follows that $\Re \cap C(E)$ contains a $S_{2^{\prime}}$-subgroup $\&$ of $\Omega$. Thus, $\mathbb{L} \subseteq \mathfrak{M}$, since $N(\langle E\rangle) \subseteq \mathfrak{M}$. Let $\Re_{2}$ be a $S_{2}$-subgroup of $\Re$ which contains $\mathfrak{I}$ and is permutable with $\mathfrak{F}_{3}$. Thus, $\mathfrak{I} \subset \Re_{2}$, since $\Re_{2} \nsubseteq \mathfrak{M}$. Let $\tilde{\Re}_{2}=\boldsymbol{O}_{2}\left(\Re_{2} \widetilde{\mho}_{3}\right)$, so that $\Re_{2} \widetilde{\mho}_{3}=\tilde{\Re}_{2} N_{\Omega_{2} \widetilde{\mho}_{3}}(\langle E\rangle)$. Since $\Re_{2} \nsubseteq \mathfrak{M}$, so also $\tilde{\Re}_{2} \nsubseteq \mathfrak{M}$. Let $\hat{\mathfrak{I}}_{0}=\tilde{\mathfrak{I}}_{2} \cap \mathfrak{I}$. Thus, $\mathfrak{I}=\hat{\mathfrak{I}}_{0} \mathfrak{Z}^{*}$, as $V^{*}$ inverts $E$ and $\left|\mathfrak{I}: \hat{\mathfrak{I}}_{0}\right| \leqq 2$. Since $\widetilde{\mathfrak{R}}_{2} \nsubseteq \mathfrak{M}$, we get $\hat{\mathfrak{I}}_{0} \cap \widetilde{\mathfrak{R}}_{2}$. Let $\tilde{\mathfrak{S}}=V\left(\operatorname{ccl}_{\mathfrak{S}}(\mathfrak{B}) ; \hat{\mathfrak{I}}_{0}\right)$. Notice that $N(\tilde{\mathfrak{W}}) \nsubseteq \mathfrak{M}$, since $\tilde{\mathfrak{W}}$ is weakly closed in $\hat{\mathfrak{I}}_{0}$ and $\widetilde{\mathfrak{I}}_{0} \subset \tilde{\mathfrak{I}}_{2}$. Since $\mathfrak{B} \subseteq \tilde{\mathfrak{B}}$, we have $\tilde{\mathfrak{B}} \neq 1$. Now $\widetilde{\mho}_{3}$ is a $S_{3}$-subgroup of $\boldsymbol{C}_{\mathfrak{R}}(X)$, so $\mathfrak{F}_{3}$ contains a $S_{3}$-subgroup of $\mathfrak{E}$. Suppose $3 \||\mathfrak{E}|$. In this case, $E \in \mathfrak{E}$. With this assumption, we will show that $\tilde{\mathfrak{B}} \subseteq \mathbb{E}$. If this containment does not hold, then since $|\mathfrak{W}: \mathfrak{W} \cap \mathfrak{C}|=2$, we get $\mathfrak{W}=(\mathfrak{W} \cap \mathfrak{C}) \tilde{\mathfrak{B}}$, which implies that $\tilde{\mathfrak{S}}$ does not centralize $\mathfrak{D}$. But $\left[\mathfrak{F}_{3}, \tilde{\mathfrak{W}}\right]$ is a 2-group contained in $\tilde{\mathfrak{I}}_{0}$ and $\mathfrak{F}_{3}$ covers $\mathfrak{D}$, so $\tilde{\mathfrak{B}}$ centralizes $\mathfrak{D}$. Thus, if 3|| $\mathfrak{F} \mid$, then $\tilde{\mathfrak{B}} \subseteq \mathbb{(}$. Since $\tilde{\mathfrak{W}}$ is normalized by $\mathfrak{F}_{3}$ and by $N_{\mathfrak{R}}(\mathfrak{F} \cap \mathfrak{T})$, it follows that $\mathfrak{R}$ normalizes $\tilde{\mathfrak{B}}$. This is impossible, since $N(\tilde{\mathfrak{B}}) \nsubseteq \mathfrak{M}$. We conclude that $3 \nmid|\mathfrak{E}|$. 
If $\tilde{\mathfrak{B}} \nsubseteq \mathfrak{E}$, then $\mathfrak{W}=(\mathfrak{W} \cap \mathfrak{C}) \tilde{\mathfrak{W}}$, since $|\mathfrak{W}: \mathfrak{W} \cap \mathfrak{F}|=2$, and so $\tilde{\mathfrak{B}}$ does not centralize $\mathfrak{D}$. This is not the case, since $\left[\tilde{\mathfrak{B}}, \mathfrak{F}_{3}\right]$ is a 2 -group and $\mathfrak{F}_{3}$ covers $\mathfrak{D}$. Hence, $\tilde{\mathfrak{B}} \subseteq \mathbb{\mathfrak { C }}$.

Since $\tilde{\mathfrak{I}}_{0}$ is the subgroup of $\mathfrak{T}$ generated by all elements of $\boldsymbol{U}_{\mathfrak{z}}(\langle E\rangle)$, it follows that $\widetilde{\mathfrak{I}}_{0}$ contains $\mathfrak{T} \cap \mathfrak{E}$. Since $\tilde{\mathfrak{B}} \subseteq \mathfrak{E}$, we get $\tilde{\mathfrak{S}}=V\left(\operatorname{ccl}_{\mathscr{\Theta}}(\mathfrak{B}) ; \tilde{\mathfrak{I}}_{0}\right)=V\left(\operatorname{ccl}_{\mathfrak{\Theta}}(\mathfrak{B}) ; \mathfrak{I} \cap \mathfrak{C}\right) \triangleleft \mathfrak{T} \mathfrak{R}$. Hence, $N(\tilde{\mathfrak{B}}) \subseteq \mathfrak{M}$. This contradiction shows that $C(X) \subseteq \mathbb{M}$.

We next show that $\sqrt{ }$ is a $3^{\prime}$-group. Suppose false and $\mathfrak{C}_{3}$ is a subgroup of $\mathfrak{E} \cap \Re$ of order 3 . Then $\mathfrak{S}_{\mathfrak{C}} \mathfrak{S}_{3} \triangleleft \mathfrak{T} \Re$, and $\boldsymbol{O}_{2}\left(\mathfrak{T} \mathfrak{S}_{3}\right)=\mathfrak{I}^{0}$ is of index 1 or 2 in $\mathfrak{I}$. Also, $N\left(\mathfrak{S}_{3}\right) \subseteq \mathfrak{M}$, by Lemma 14.22. Let $\&$ be a $S_{2,3}$-subgroup of $\boldsymbol{C}(3)$ which contains $\mathfrak{T C}_{3}$ and let $\left\{\mathbb{R}_{2}, \mathbb{R}_{3}\right\}$ be a Sylow system of $\mathbb{R}$ with $\mathfrak{I} \subseteq \mathfrak{R}_{2}, \mathfrak{E}_{3} \subseteq \mathfrak{R}_{3}$. If $\mathfrak{\Omega}_{3}$ is non cyclic, then so is $\mathfrak{R}_{3} \cap \mathfrak{M}$, so $C(3) \subseteq \mathfrak{M}$, by Lemma 14.20 . This is not the case, so $\mathfrak{Z}_{3}$ is cyclic. Hence, $\mathfrak{I}_{0} \subseteq O_{2}(\mathbb{R})$, and $\mathfrak{\Omega}_{3} \subseteq N\left(\mathfrak{C}_{3}\right) \subseteq \mathfrak{M}$.

By Lemma 15.6, we get $\mathfrak{I}^{0} \subset \mathfrak{T}$. Since $\mathfrak{I}^{0} \subset \mathfrak{T}$, we get $\mathfrak{S}_{3} \leqq C(3)^{\prime}$. By Lemma 14.22, together with $\sigma_{2}=\{3\}$, we conclude that $C(\mathbb{Z}) \cap \mathfrak{M}$ contains a $S_{2^{\prime}}$-subgroup of $C(3)$. Hence, $\mathfrak{I} \subset \mathfrak{R}_{2}$. Since $\mathfrak{\Omega}_{2}=\boldsymbol{O}_{2}(\mathbb{R}) \boldsymbol{N}_{\mathbb{R}_{2}}\left(\mathfrak{S}_{3}\right)$, it follows that $O_{2}(\mathbb{R}) \nsubseteq \mathfrak{M}$, so $\mathfrak{T}^{0} \subset \boldsymbol{O}_{2}(\mathbb{R})$. Let $\mathfrak{T}^{1} / \mathfrak{T}^{0}$ be a chief factor of $\boldsymbol{O}_{2}(\mathbb{R}) \mathfrak{C}_{3}$ with $\mathfrak{T}^{1} \subseteq \boldsymbol{O}_{2}(\mathbb{R})$. Since $N\left(\mathfrak{C}_{3}\right) \subseteq \mathfrak{M}$, it follows that $\mathfrak{I}^{1} \mathfrak{C}_{3} / \mathfrak{T}^{0} \cong A_{4}$. On the other hand, $\mathfrak{S}_{3}$ centralizes $Z(\mathfrak{T})$, and so centralizes $Z\left(\mathfrak{T}^{\circ}\right)$. Hence, $\mathfrak{I}^{1}$ centralizes $\boldsymbol{Z}(\mathfrak{T})$. This is not the case, since $\boldsymbol{C}(X) \subseteq \mathfrak{M}$, for all $X$ in $Z(\mathfrak{I})^{*} \cap \mathfrak{B}^{\circ}$. We conclude that $\mathfrak{F}$ is a $3^{\prime}$-group.

We next show that $\mathfrak{I} / \tilde{\mathfrak{Z}}$ is elementary of order 4 . Suppose false. Then $|\mathfrak{T}: \mathfrak{S}|=2$ and $\mathfrak{I}=\mathfrak{S C}_{2} *$. Since $\left[\Re, \mathfrak{B}^{*}\right] \mathfrak{S} / \mathfrak{S}$ is of order 3 , it follows that $\Re \nsubseteq \mathfrak{M}^{\prime}$, against Lemma 15.6. Hence, $\mathfrak{I} / \mathfrak{F}$ is elementary of order 4.

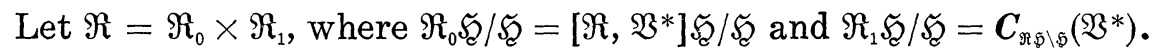

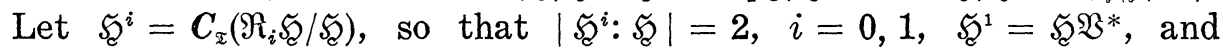
$\mathfrak{T} / \mathfrak{S}=\mathfrak{S}^{0} / \mathfrak{S E} \times \mathfrak{S}^{1} / \mathscr{S}$. Since $3 \nmid|\mathfrak{E}|$, it follows that $\left|\Re_{0}\right|=3$, and that $\Re_{1}$ centralizes $\mathfrak{B}^{1}$, as does $\mathfrak{S}^{0}$.

Let $\mathfrak{Q}=\mathfrak{I R}_{1}$. Then $\boldsymbol{O}_{2}(\mathfrak{Q})=\mathfrak{S}^{1}$, and $\mathfrak{S C}^{1}$ does not centralize $\mathfrak{B}^{1}$. Suppose $\mathfrak{U}$ is a four-group contained in $Z\left(\mathfrak{S}^{1}\right), \mathfrak{U} \triangleleft \mathfrak{I}$, and $Z \in \mathfrak{U}$. We argue that $\mathfrak{U} \subseteq Z(\mathfrak{I})$. Namely, choose $U \in \mathfrak{U}-\langle Z\rangle$. Then $U=U^{0} U^{1}$ with $U^{i} \in \mathfrak{B}^{i}$. Since $\mathfrak{B}^{*} \subseteq \mathfrak{S}^{1}$, we get $\left[U, \mathfrak{B}^{*}\right]=1$. Since $\mathfrak{B}^{i}$ admit $\mathfrak{T}$, it follows that $\left[U^{i}, \mathfrak{B}^{*}\right]=1$. Hence, $U^{1} \in \mathbb{Z} \subseteq Z(\mathfrak{I})$. Hence, $[U, \mathfrak{I}] \subseteq$ $3 \cap \mathfrak{B}^{0}=1$, so the assertion holds.

Since $\Re_{1}$ centralizes $\mathfrak{B}^{1}$ and $\Re_{0}$ does not, it follows from Lemma 14.22 that $N\left(\Omega_{1}\left(\Re_{1}\right)\right) \subseteq \mathfrak{M}$, and since $\Re \subseteq \mathfrak{M}^{\prime}$, we get $\Re_{1} \subseteq N\left(\Omega_{1}\left(\Re_{1}\right)\right)^{\prime}$.

Let $\Re=C(3) \supseteq \mathfrak{T}_{1}$. Since $\Re \nsubseteq \mathfrak{M}, S_{3}$-subgroups of $\Re$ are cyclic, so $\Re_{1}$ is a $S_{3}$-subgroup of $\Re$. Let $\&$ be a $S_{2,3}$-subgroup of $\Re$ which contains $\mathfrak{T}_{1}$ and let $\mathbb{R}_{2}$ be a $S_{2}$-subgroup of $\mathbb{R}$ which contains $\mathfrak{T}$. Lemma 14.22 implies that $\Omega \cap \mathfrak{M}$ contains a $S_{2}$-subgroup of $\Re$, so $\mathfrak{R}_{2} \subseteq \mathfrak{M}$. Let $\mathfrak{R}_{1}=\boldsymbol{O}_{2}(\mathbb{R}) \supseteqq \mathfrak{S}^{1}$. Let $\mathfrak{S}^{2} / \mathfrak{S}^{1}$ be a chief factor of $\mathfrak{R}_{2} \Omega_{1}\left(\mathfrak{R}_{1}\right) \cdot \mathfrak{T}$ 
with $\mathfrak{S}^{2} \cong \mathfrak{R}_{1}$. Since $N\left(\Omega_{1}\left(\Re_{1}\right)\right) \subseteq \mathfrak{M}$, it follows that $\mathfrak{\Omega}_{2} \Omega_{1}\left(\Re_{1}\right) / \mathfrak{S}^{1} \cong A_{4}$, $\mathfrak{\Omega}_{2} \Omega_{1}\left(\Re_{1}\right) \cdot \mathfrak{T} / \mathfrak{S}^{1} \cong \sum_{4}$.

Let $\mathfrak{X}=\Omega_{1}\left(\boldsymbol{Z}\left(\mathfrak{S}^{1}\right)\right)$, and let $\mathfrak{X}_{0}=\mathfrak{X} \cap \boldsymbol{C}\left(\mathfrak{S C}^{2}\right) \supseteqq \mathfrak{3}$. If $\mathfrak{X}_{0} \supset \mathfrak{Z}$, let $\mathfrak{U}$ be a four-subgroup of $\mathfrak{X}_{0}$ with $\mathfrak{U} \supset \mathfrak{Z}, \mathfrak{U} \triangleleft \mathfrak{T}$. By a previous argument, we get $\mathfrak{u} \subseteq Z(\mathfrak{Z})$. Hence, $\mathfrak{U} \cap \mathfrak{B}^{0} \neq 1$. Choose $U \in \mathfrak{U} \cap \mathfrak{B}^{0}, \mathfrak{U} \neq 1$. By a previous argument $\boldsymbol{C}(U) \subseteq \mathfrak{M}$, against $\mathfrak{S}^{2} \nsubseteq \mathfrak{M}$. Hence, $\mathfrak{X}_{0}=\mathfrak{Z}$.

Since $\mathfrak{X} \supseteqq \Omega_{1}(\boldsymbol{Z}(\mathfrak{I}))$, it follows that $\mathfrak{X} \supset \mathfrak{X}_{0}$. Let $\mathfrak{X}_{1} / \mathfrak{X}_{0}$ be a chief factor of $\mathfrak{S}_{2} \Omega_{1}\left(\Re_{1}\right) \mathfrak{I}$ with $\mathfrak{X}_{1} \subseteq \mathfrak{X}$. Then $\left[\mathfrak{S}^{2}, \mathfrak{X}_{1}\right] \subseteq \mathfrak{X}_{0}$, since $\mathfrak{S}_{2} \triangleleft \mathfrak{R}_{2} \Re_{1}$. Let $\mathfrak{X}_{2}$ be a four-subgroup of $\mathfrak{X}_{1}$ which contains 3 and admits $\mathfrak{T}$. By a previous argument $\mathfrak{X}_{2} \leqq Z(\mathfrak{T})$. Choose $X \in \mathfrak{X}_{2} \cap \mathfrak{B}^{0}, X \neq 1$. Then $C(X) \subseteq \mathfrak{M}$, by a previous argument. Hence, $C_{\mathfrak{F}^{2}}(X)=\mathfrak{S}^{1}$, since $\mathfrak{S C}^{2} \cap$ $\mathfrak{M}=\mathfrak{S}^{1}$. On the other hand, $\mathfrak{S}^{2}$ stabilizes the chain $\mathfrak{X}_{2} \supset 3 \supset 1$, so $\boldsymbol{C}_{\mathfrak{1} 2}(X)$ is of index at most 2 in $\mathfrak{S}_{\mathfrak{C}}{ }^{2}$. This contradiction completes the proof.

LEMMA 15.12.

(a) $|\Re|=9$.

(b) $\mathfrak{I} / \mathfrak{S}$ is dihedral of order 4 or 8.

(c) $|\mathfrak{W}: \mathfrak{W} \cap \mathbb{E}|=4$.

(d) $3 \nmid|\mathfrak{c}|$.

Proof. By Lemma 15.11, $|\mathfrak{W}: \mathfrak{W} \cap \mathbb{E}| \geqq 4$. Choose $G \in \mathbb{B}$, with $\mathfrak{B}^{*}=\mathfrak{B}^{G} \subseteq \mathfrak{I}, \mathfrak{B}^{*} \nsubseteq \mathfrak{C}$, and let $\mathfrak{B}_{0}^{*}=\mathfrak{B}^{*} \cap \mathfrak{C}$. By Lemma $15.10, \mathfrak{B}_{0}^{*}$ is a hyperplane of $\mathfrak{B}^{*}$, so by Lemma $15.1, \mathfrak{V} \subseteq \mathfrak{M}^{\alpha}$. Let $3=\left[\mathfrak{B}, \mathfrak{B}^{*}\right]$ so that $3=\langle Z\rangle$ is of order 2 . Choose $V^{*} \in \mathfrak{B}^{*}-\mathfrak{B}_{0}^{*}$, let $\Re=\mathfrak{I} \Re$ and for each subset $\mathfrak{S}$ of $\mathbb{R}$, let $\overline{\mathfrak{S}}=\mathfrak{S} \mathscr{F} / \mathfrak{S}$. Thus, $\boldsymbol{O}_{2}(\overline{\mathfrak{L}})=1$. Since $3 \in \pi_{2}$, it follows that $\overline{\mathfrak{I}}$ is isomorphic to a subgroup of $G L(2,3)$. By Lemma 15.3, $\bar{\Re} \triangleleft \overline{\mathfrak{L}}$. Thus, $\mathfrak{B}^{*}$ normalizes $\mathfrak{S} \Re$.

Since a $S_{2}$-subgroup of $\boldsymbol{O}_{3^{\prime}}(\mathfrak{M})$ is a $Z$-group, it follows that

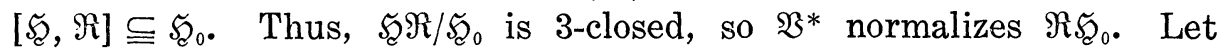
$\mathfrak{X}=\left\langle\mathfrak{B}^{*}, \mathfrak{R}, \mathfrak{S}_{0}\right\rangle / \mathfrak{S}_{0_{0}}$. We may view $\mathfrak{B}$ as a $\mathfrak{X}$-module since $\mathfrak{B} \leqq Z\left(\mathfrak{S}_{0}\right)$. Let $\Re_{1}=\left[\Re, \mathfrak{B}^{*}\right] \mathfrak{\mathcal { C }}_{0} / \mathfrak{S}_{0}, \quad \Re_{2}=\boldsymbol{C}_{\mathfrak{r} \mathfrak{F}_{0} / \mathfrak{F}_{0}}\left(\mathfrak{B}^{*}\right)$, and let $\mathfrak{B}^{0}=\boldsymbol{C}_{\mathfrak{B}}\left(\Re_{1}\right), \quad \mathfrak{B}^{1}=$ $\left[\mathfrak{B}, \mathfrak{R}_{1}\right]$. Thus, $\left|\mathfrak{B}^{1}\right|=4$, and $3 \subset \mathfrak{B}^{1}$. Since $B=\left[\mathfrak{B}, \mathfrak{B}^{*}\right]$, it follows that $\Re_{2}$ centralizes 3 , and also $C_{\Re_{1}}(3)$ is of index 3 in $\Re_{1}$. Hence, $C_{\Re}(3)$ is of index 3 in $\Re$.

Suppose $\boldsymbol{C}_{\mathfrak{R}}(3)$ is non cyclic. In this case, $\boldsymbol{C}(3) \subseteq \mathfrak{M}$, by Lemma 14.20. In particular, $C_{\mathfrak{M} G}(3) \subseteq \mathfrak{M}$. But $\boldsymbol{C}_{\mathfrak{M} G}(3)$ contains $\boldsymbol{O}_{2}\left(\mathfrak{M}^{G}\right)$ and also contains a subgroup $\mathfrak{X}^{*}$ of order 3 with $N\left(\mathfrak{X}^{*}\right) \subseteq \mathfrak{M}^{G}$, by symmetry. Hence, $\mathfrak{M} \cap \mathfrak{M}^{G}$ has non cyclic $S_{3}$-subgroups, against Lemma 14.20. We conclude that $C_{\Re}(3)$ is cyclic. Hence, $\Re$ is metacyclic, so is abelian, by 0.3 .8 . More precisely, $\Re$ has a cyclic subgroup of index 3.

We next argue that $\left|\Re_{1}\right|=3$. Suppose $\left|\Re_{1}\right| \geqq 9$. In this case, $\boldsymbol{C}_{\mathfrak{r}}(\mathfrak{3}) \mathfrak{H}_{\mathcal{C}} / \mathscr{S}_{\mathcal{E}}$ contains elements of order 3 which are inverted by $\mathfrak{S}^{*}$ 
and also contains elements of order 3 which are centralized by $V^{*}$, so that $\boldsymbol{C}_{\mathfrak{r}}(3)$ is non cyclic. Hence, $\left|\Re_{1}\right|=3$, and so $\mathfrak{B}^{*}$ centralizes $\mathfrak{S}_{0} \mathfrak{R} / \mathfrak{S}_{0} \Omega_{1}(\Re)$.

Since $\Re^{\prime}=1$, we get $\mathfrak{S}_{0} \Re \triangleleft \mathfrak{T} \Re$, and we may view $\mathfrak{S}_{0} \Re / \mathscr{C}_{0}$ as a $\mathfrak{I} / \mathfrak{S}$-group. Since $\mathfrak{B S} / \mathscr{S E}$ is generated by involutions, and since $\mathfrak{T} / \mathfrak{F}$ acts faithfully on $\mathfrak{S}_{0} \mathfrak{R} / \mathscr{F}_{0}$, it follows that $\mathfrak{W} \mathfrak{F}_{\mathcal{E}} / \mathfrak{S}_{\mathcal{C}}$ is dihedral. We argue that $\mathfrak{W}^{\prime} \subseteq \mathfrak{C}$. Namely, $\mathfrak{W}$ is generated by involutions which centralize hyperplanes of $\mathfrak{B}$. Suppose $I_{1}, I_{2} \in \mathfrak{M}, C_{\mathfrak{B}}\left(I_{i}\right)=\mathfrak{B}_{i}, i=1,2$, and $\mathfrak{B}_{i}$ is a hyperplane of $\mathfrak{B}$. If $\left[I_{1}, I_{2}\right] \notin \mathbb{E}$, then $\left\langle I_{1}, I_{2}\right\rangle \mathbb{E} / \mathbb{E}$ is dihedral of order 8. This implies that $\left\langle I_{1}, I_{2}\right\rangle \mathbb{E} / \mathbb{E}$ is represented faithfully on $\boldsymbol{O}_{3}(\mathfrak{M} / \mathfrak{S})$, so $3 \nmid|\mathfrak{S}|$, and $\Omega_{1}(\mathfrak{R}) \mathfrak{S} / \mathfrak{S} \triangleleft \mathfrak{M} / \mathfrak{S}$. Now Lemma 5.47 gives a contradiction, since $\left(I_{1} I_{2}\right)^{2}$ also centralizes a hyperplane of $\mathfrak{B}$. We conclude that (c) holds, and that (b) holds, by Lemma 15.3 and Lemma 15.11.

Since $\mathfrak{W C} / \mathbb{C}$ is a four-group and is represented faithfully on $O_{3}(\mathfrak{M} / \mathfrak{S})$, and since $\mathfrak{M} \mathbb{C} / \mathfrak{S}$ centralizes $\Re \mathfrak{C} / \Omega_{1}(\mathfrak{R}) \mathfrak{C}$, it follows that $|\Re|=9$ and that $3 \nmid|\mathfrak{C}|$. The proof is complete.

LEMMA 15.13. $\mathfrak{M}=\mathfrak{T} \mathfrak{R}$.

Proof. Suppose false. Thus, $\mathfrak{M}$ is not a 2,3 -group. Let $\mathfrak{D}$ be a $S_{\{2,3\}}$-subgroup of $\mathfrak{M}$ which is permutable with $\mathfrak{I}$, and with $\mathfrak{R}$. Since $\mathfrak{D}$ is a $Z$-group, and since $\mathfrak{D} \triangleleft \mathfrak{D} \Re$, and since $\mathfrak{R} \subseteq \mathfrak{M}^{\prime}$, it follows that $[\mathfrak{D}, \Re]=1$.

Choose $G$ in $(S)$ such that $\mathfrak{B}^{*}=\mathfrak{B}^{G} \subseteq \mathfrak{I}, \mathfrak{B}^{*} \nsubseteq \mathfrak{S}$, and let $\mathfrak{B}_{0}^{*}=$ $\mathfrak{B}^{*} \cap \mathfrak{E}$, a hyperplane of $\mathfrak{B}^{*}$. Let $\mathfrak{R}_{0}=\left[\mathfrak{R}, \mathfrak{B}^{*}\right] \mathfrak{\mathcal { E }} \cap \mathfrak{R}$, so that $\left|\mathfrak{R}_{0}\right|=3$. Let $\mathfrak{B}=\mathfrak{B}^{0} \times \mathfrak{B}^{1}$, where $\mathfrak{B}^{0}=\boldsymbol{C}_{\mathfrak{B}}\left(\mathfrak{R}_{0}\right), \mathfrak{B}^{1}=\left[\mathfrak{B}, \Re_{0}\right]$, so that $\mathfrak{B}^{1}$ is a

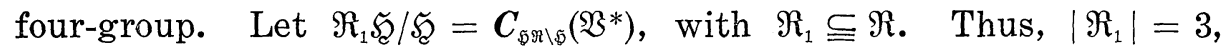
$\Re=\Re_{0} \times \Re_{1}$ and $\Re_{1} \mathfrak{D}$ centralizes $\mathfrak{B}^{1}$.

By Lemma 14.5, D is a $S$-subgroup of $(B), \pi(D) \leqq \pi_{1}$, and by Lemma 6.2, DD $\subseteq \mathfrak{M}^{\prime}$. Hence, $\mathfrak{D}$ is cyclic. Since $\left|\mathfrak{B}^{1}\right|=4$, it follows that $N\left(\mathfrak{D}_{0}\right) \subseteq \mathfrak{M}$ for every non identity subgroup $\mathfrak{D}_{0}$ of $\mathfrak{D}$. We omit the details of this argument, which is an easy consequence of Lemma 14.13.

Let $3=\left[\mathfrak{B}, \mathfrak{B}^{*}\right]=\left[\mathfrak{B}^{1}, \mathfrak{B}^{*}\right]=\langle Z\rangle$. Thus, $3 \subseteq \mathfrak{B}^{*}$, and $C_{\mathfrak{R} G}(3)$ contains a subgroup $\mathfrak{X}^{*}$ of order 3 with $N\left(\mathfrak{I}^{*}\right) \subseteq \mathfrak{M}^{G}$. Hence, $C(3)=$ $\Re \nsubseteq \mathfrak{M}$.

Since $\Re_{1} \mathfrak{D} \subseteq \Re$ and $N\left(\Re_{1}\right) \subseteq \mathfrak{M}$, and $\mathfrak{R}_{1} \subseteq \mathfrak{M}^{\prime}$, it follows that $\mathfrak{R}_{1} \mathfrak{D}$ is a $S_{2}$-subgroup of $\Re$. Let $\Re_{2}$ be a $S_{2}$-subgroup of $\Re$ which contains $\boldsymbol{C}_{\mathfrak{\Sigma}}(3)$ and is permutable with $\Re_{1}$ and with $\mathfrak{D}$. Let $\Re_{1}=\boldsymbol{O}_{2}(\Re), \mathfrak{I}_{1}=$ $\Re_{1} \cap C_{\mathfrak{x}}(3)$. Thus, $\mathfrak{I}_{1}=\mathfrak{I}_{1} \cap \mathfrak{M}$, and $\mathfrak{I}_{1} \subset \mathfrak{\Re}_{1}$. Let $\mathfrak{I}_{2} / \mathfrak{I}_{1}$ be a chief factor of $\Re$ with $\mathfrak{I}_{2} \subseteq \Re_{1}$. Then $\mathfrak{I}_{2} \mathfrak{D} \Re_{1} / \mathfrak{I}_{1}$ is a Frobenius group, so $\left|\mathfrak{I}_{2}: \mathfrak{I}_{1}\right| \geqq 16$.

Let $\tilde{\mathfrak{B}}=[\mathfrak{B}, \mathfrak{R}]$. Since $|\mathfrak{W}: \mathfrak{W} \cap \mathfrak{E}|=4$, we get $|\tilde{\mathfrak{B}}|=16$, and 
$\mathfrak{B}=\hat{\mathfrak{B}} \times \hat{\mathfrak{B}}$, where $\hat{\mathfrak{B}}=\boldsymbol{C}_{\mathfrak{B}}(\mathfrak{R})$. Choose $G_{1}$ in (3) such that $\mathfrak{W} \mathfrak{F}=$ $\left\langle\mathfrak{S}_{\mathfrak{e}}, \mathfrak{B}^{*}, \mathfrak{B}^{G_{1}}\right\rangle$. Then $\tilde{\mathfrak{B}}=\left(\mathfrak{B}^{0} \cap \tilde{\mathfrak{B}}\right) \times \mathfrak{B}^{1}$, and $\boldsymbol{C}_{\mathfrak{x}}(\mathbb{3})=\mathfrak{S}_{\mathfrak{S}} \mathfrak{W}$, so that $\mathfrak{I}_{1}=$ $\mathfrak{S C B}^{*}$. This implies that $\Omega_{1}\left(\mathbb{Z}\left(\mathfrak{I}_{1}\right)\right)=\left(\Omega_{1}\left(\boldsymbol{Z}\left(\mathfrak{I}_{1}\right)\right) \cap \hat{\mathfrak{B}}\right) \times 3 \times\left(\mathfrak{B}^{\circ} \cap \tilde{\mathfrak{B}}\right)$. Since $\mathfrak{R}$ centralizes $\hat{\mathfrak{B}}$, we get $\boldsymbol{C}(X) \subseteq \mathfrak{M}$ for all $X$ in $\hat{\mathfrak{S}}^{\sharp}$. Hence, $\boldsymbol{Z}\left(\mathfrak{I}_{2}\right) \cap \widehat{\mathfrak{B}}=1$. Since $\Omega_{1}\left(\boldsymbol{Z}\left(\mathfrak{I}_{1}\right)\right)=\left(\Omega_{1}\left(\boldsymbol{Z}\left(\mathfrak{I}_{1}\right)\right) \boldsymbol{C}(\mathfrak{R})\right) \times\left[\tilde{\mathfrak{B}}, \mathfrak{R}_{1}\right]$, and since $\left[\tilde{\mathfrak{B}}, \Re_{1}\right]$ is a four-group, it follows that either $\Omega_{1}\left(\boldsymbol{Z}\left(\mathfrak{I}_{2}\right)\right) \cap\left[\tilde{\mathfrak{B}}, \Re_{1}\right]=1$, or $\Omega_{1}\left(\boldsymbol{Z}\left(\mathfrak{I}_{2}\right)\right) \supseteqq\left[\tilde{\mathfrak{B}}, \mathfrak{R}_{1}\right]$. If $\Omega_{1}\left(\boldsymbol{Z}\left(\mathfrak{I}_{2}\right)\right) \supseteq\left[\tilde{\mathfrak{B}}, \mathfrak{R}_{1}\right]$, we get $N\left(\left[\tilde{\mathfrak{B}}, \mathfrak{R}_{1}\right]\right) \nsubseteq \mathfrak{M}$, against $\Re \mathfrak{S}_{0} \subseteq N\left(\left[\tilde{\mathfrak{B}}, \Re_{1}\right]\right)$. Hence, $\Omega_{1}\left(\boldsymbol{Z}\left(\mathfrak{I}_{2}\right)\right) \cap\left[\tilde{\mathfrak{B}}, \Re_{1}\right]=1$, so that $\Omega_{1}\left(\boldsymbol{Z}\left(\mathfrak{I}_{2}\right)\right) \leqq 3 \times\left(\Omega_{1}\left(\boldsymbol{Z}\left(\mathfrak{I}_{2}\right)\right) \cap \widehat{\mathfrak{B}}\right)$. This implies that $\Omega_{1}\left(\boldsymbol{Z}\left(\mathfrak{I}_{2}\right)\right)=3$.

Let $\mathfrak{x} / 3$ be a chief factor of $\mathfrak{I}_{2} \Re_{1} \mathfrak{D}$ with $\mathfrak{X} \subseteq \mathbb{Z}\left(\mathfrak{I}_{1}\right)$. Thus, $\mathfrak{X} \supseteqq\left[\tilde{\mathfrak{B}}, \mathfrak{R}_{1}\right]$ or $\mathfrak{X} \cap \widehat{\mathfrak{B}} \neq 1$. Both possibilities give contradictions, since $\mathfrak{I}_{2}$ stabilizes $\mathfrak{x} \supset \supset 1$ and $\left|\mathfrak{I}_{2}: \mathfrak{I}_{1}\right| \geqq 16$. The proof is complete.

We now introduce the following notation. Choose $G_{1}, G_{2}$ in (S) such that $\mathfrak{S} \mathfrak{W}=\left\langle\mathfrak{F}_{\mathcal{E}}, \mathfrak{B}^{G_{1}}, \mathfrak{B}^{\alpha_{2}}\right\rangle$. Let $\mathfrak{X}_{i}=\mathfrak{B}^{G_{i}}, i=1,2$. Write $\mathfrak{R}=$ $\Re_{1} \times \Re_{2}$, where $\Re_{i} \mathfrak{S} / \mathscr{S}=C_{\mathfrak{S} \Re \mathfrak{S}}\left(\mathfrak{X}_{i}\right), i=1,2$. Choose $V_{i} \in \mathfrak{X}_{i}-\mathfrak{S}, i=1,2$. Thus, $V_{i}$ inverts $\mathfrak{S}_{\mathfrak{B}} \mathfrak{R}_{3-i} / \mathfrak{S}$. Write $\mathfrak{B}=\mathfrak{B}^{0} \times \mathfrak{B}^{1}$, where $\mathfrak{S}^{0}=\boldsymbol{C}_{\mathfrak{B}}(\mathfrak{R})$, $\mathfrak{B}^{1}=[\mathfrak{B}, \mathfrak{R}]$, so that $\left|\mathfrak{B}^{1}\right|=16$. Let $\mathfrak{B}_{i}^{1}=\left[\mathfrak{B}, \mathfrak{R}_{i}\right], i=1$, 2 , so that $\mathfrak{B}^{1}=$ $\mathfrak{B}_{1}^{1} \times \mathfrak{B}_{2}^{1}$, and $\Re_{i}$ centralizes $\mathfrak{B}_{3-i}^{1}, i=1,2$. Since $V_{i}$ inverts $\mathfrak{S}_{\mathfrak{C}} \Re_{3-i} / \mathfrak{S}$, it follows that $\left[\mathfrak{X}_{i}, \mathfrak{B}\right]=\left[\mathfrak{X}_{i}, \mathfrak{V}_{3-i}^{1}\right]=\left\langle Z_{3-i}\right\rangle, i=1,2$. Since $Z_{3-i} \in \mathfrak{X}_{i}$, it follows that $C\left(Z_{3-i}\right) \nsubseteq \mathfrak{M}, i=1,2$. Since $\Re_{i}$ centralizes $Z_{3-i}$, as does $\mathfrak{W}$, we get $\Re_{i} \subseteq C_{\mathfrak{M}}\left(Z_{3-i}\right)^{\prime}$. Let $\Re_{i}=C\left(Z_{3-i}\right), i=1,2$. Since $N\left(\Re_{i}\right) \subseteq \mathfrak{M}$, it follows that $\mathfrak{R}_{i}=\mathfrak{R}_{i} \mathfrak{I}_{i}$, where $\mathfrak{I}_{i}$ is a $S_{2}$-subgroup of $\Re_{i}$ which contains $C_{\mathfrak{z}}\left(Z_{3-i}\right)$. Furthermore, $C_{\mathfrak{z}}\left(Z_{3-i}\right)=\mathfrak{F} \mathfrak{B}$, and $|\mathfrak{I}: \mathfrak{F} \mathfrak{W}| \leqq 2$. Final-

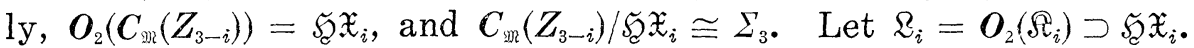

LeMma 15.14. If $i \in\{1,2\}$, then $\left|N_{\Sigma_{i}}\left(\mathfrak{S}_{\mathfrak{X}} \mathfrak{X}_{i}\right): \mathfrak{S}_{\mathfrak{X}} \mathfrak{X}_{i}\right|=4$, and $\left\langle Z_{3-i}\right\rangle=$ $\Omega_{1}\left(\boldsymbol{Z}\left(\boldsymbol{N}_{\Omega_{i}}\left(\mathfrak{S}_{\mathfrak{e}} \mathfrak{X}_{i}\right)\right)\right)$.

Proof. A repetition of the argument of Lemma 15.13 yields the assertions of this lemma.

Lemma 15.15 . $\mathfrak{I} / \mathfrak{S}$ is dihedral of order 8.

Proof. Let $\mathbb{S}^{i}$ be a $S_{2}$-subgroup of $\mathbb{S}$ which contains $\boldsymbol{N}_{\Omega_{i}}\left(\mathfrak{S}_{2} \mathfrak{X}_{i}\right)$. Then $\Omega_{1}\left(\boldsymbol{Z}\left(\mathbb{S}^{i}\right)\right) \subseteq \mathbb{Z}\left(\boldsymbol{N}_{\varepsilon_{i}}\left(\mathfrak{S}_{\mathcal{C}} \mathfrak{X}_{i}\right)\right)$, so we get $\left\langle Z_{3-i}\right\rangle=\Omega_{1}\left(\boldsymbol{Z}\left(\mathbb{S}^{i}\right)\right)$. Hence, $C\left(Z_{3-i}\right)=\mathbb{B}_{3} i \Re_{i}, i=1,2$. Since $Z_{1} \sim Z_{2}$, it follows that $\Re_{1} \sim \Re_{2}$, and so $\Re_{1}$ and $\Re_{2}$ are $N(\Re)$-conjugate. It suffices to show that $\Re_{1}$ and $\Re_{2}$ are $\mathfrak{M}$-conjugate. Suppose false. Let $\mathfrak{I}^{*}=N_{\mathfrak{x}}(\mathfrak{R})$. Thus, $\mathfrak{I}^{*} / \boldsymbol{C}_{\mathfrak{x}^{*}}(\mathfrak{R})$ is a four-group, and $\Re_{1}$ and $\Re_{2}$ are the only subgroups of $\Re$ of order 3 which admits $\mathfrak{T}^{*}$. Hence, $\boldsymbol{N}_{\mathbb{A}_{(}(\Re)}\left(\boldsymbol{A}_{\mathfrak{R}^{*}}(\Re)\right)$ permutes $\left\{\Re_{1}, \Re_{2}\right\}$. Since $\boldsymbol{C}_{\mathfrak{5}}\left(\Re_{i}\right) \neq 1, \quad i=1,2$, it follows that $\Re_{1} \Re_{2}$, by Lemma 14.9. The proof is complete.

TheOREM 15.1. If $|\mathfrak{B}|>8$, then $\mathfrak{I}$ is a $S_{2}$-subgroup of (3). 
Proof. Suppose false. We retain the preceding notation. Set $\mathfrak{R}_{i}=N_{\varepsilon_{i}}\left(\mathfrak{S}_{\mathcal{C}} \mathfrak{X}_{i}\right)$, so that $\mathfrak{N}_{i} / \mathfrak{S}_{\mathfrak{X}}$ is a four-group on which $\mathfrak{W}$ acts non trivially. Let $\mathfrak{Y}_{i}=\Omega_{1}\left(\boldsymbol{Z}\left(\mathfrak{S C X}_{i}\right)\right)$, so that $\mathfrak{Y}_{i}=\mathfrak{B}^{0} \times \mathfrak{B}_{i}^{1} \times\left\langle Z_{3-i}\right\rangle$. Let $\tilde{\mathfrak{V}}_{i}=\mathfrak{Y}_{i} \cap \boldsymbol{Z}\left(\mathfrak{N}_{i}\right)$. Since $N\left(\mathfrak{B}_{i}^{1}\right) \subseteq \mathfrak{M}$, it follows that $\mathfrak{B}_{i}^{1} \nsubseteq \tilde{\mathfrak{Y}}_{i}$. Hence, $\mathfrak{B}_{i}^{1} \cap \tilde{\mathfrak{Y}}_{i}=1$, since $\left|\mathfrak{B}_{i}^{1}\right|=4$ and $\mathfrak{B}_{i}^{1}=\left[\mathfrak{B}_{i}^{1}, \mathfrak{R}_{i}\right]$. Since $\tilde{\mathfrak{Y}}_{i}$ admits $\Re_{i}$, we get $\tilde{\mathfrak{V}}_{i}=\left\langle Z_{3-i}\right\rangle$ (as we have already shown earlier). It follows that $\left|\mathfrak{B}^{0}\right| \leqq 2$, and if $\left|\mathfrak{B}^{0}\right|=2$, then $\mathfrak{Y}_{i}$ is a free $F_{2} \mathfrak{N}_{i} / \mathfrak{S}_{\mathfrak{C}} \mathfrak{X}_{i}$-module. In any case, $\mathfrak{N}_{i}$ normalizes $\mathfrak{B}_{i}^{1} \times\left\langle Z_{3-i}\right\rangle$.

Set $\mathfrak{U}=\left\langle Z_{1}, Z_{2}\right\rangle$. Thus, $\mathfrak{R}_{i}$ stabilizes the chain $\mathscr{C}_{i}: \mathfrak{U} \supset\left\langle Z_{3-i}\right\rangle \supset 1$, so $\boldsymbol{C}_{\mathfrak{\Re}_{i}}(\mathfrak{U})$ is of index 2 in $\mathfrak{R}_{i}$ and $\mathfrak{R}_{i}$ maps onto $\boldsymbol{A}\left(\mathscr{C}_{i}\right), i=1,2$.

We next show that $\boldsymbol{N}_{\Theta^{i} \Re_{i}}(\mathfrak{S} \mathfrak{W})=\mathfrak{S} \mathfrak{F} \cdot C_{\mathfrak{R}_{i}}(\mathfrak{U})$. Let $\tilde{\mathfrak{N}}_{i}=N_{\Theta^{i} \mathfrak{\Re}_{i}}(\mathfrak{S} \mathfrak{Z})$. Since $\mathfrak{W}$ does not centralize $\mathfrak{R}_{i} \mathfrak{R}_{i} / \mathbb{R}_{i}$, it follows that $\tilde{\mathfrak{N}}_{i}$ is a 2-group, and so $\tilde{\mathfrak{R}}_{i}=\mathfrak{S} \mathfrak{W}\left(\tilde{\mathfrak{N}}_{i} \cap \mathfrak{R}_{i}\right)$. Thus, $\tilde{\mathfrak{N}}_{i}$ normalizes $\mathfrak{S} \mathfrak{B} \cap \mathfrak{R}_{i}=\mathfrak{S} \mathfrak{X}_{i}$, so $\tilde{\mathfrak{N}}_{i} \subseteq \mathfrak{R}_{i}$. Since $\mathfrak{N}_{i} / \mathfrak{S}_{\mathfrak{C}} \mathfrak{W}_{i}$ is a four-group on which $\mathfrak{W}$ acts non trivially, we get $\tilde{\mathfrak{R}}_{i} \subseteq \boldsymbol{C}_{\mathfrak{R}_{i}}(\mathfrak{U})$, as $\boldsymbol{C}_{\mathfrak{\Re}_{i}}(\mathfrak{U})$ is the only subgroup of $\mathfrak{N}_{i}$ of index 2 which contains $\mathfrak{S E X}_{i}$ and admits $\mathfrak{S} \mathfrak{W}$. Thus, the assertion holds.

Since $\mathfrak{I} / \mathfrak{S}$ is dihedral of order 8 , we can choose $T \in \mathfrak{T}-\mathfrak{S} \mathfrak{B}$. Thus, $T$ normalizes $\mathfrak{U}$ and normalizes $\mathfrak{S} \mathfrak{E}$, and $Z_{1}^{T}=Z_{2}$. Hence, $N_{\Theta^{1} \Re_{1}}(\mathfrak{S} \mathfrak{C})^{T}=N_{\Theta^{2} \Re_{2}}(\mathfrak{F} \mathfrak{C})$. But $N_{\Theta^{2} \Re_{2}}(\mathfrak{S} \mathfrak{C})$ centralizes $\mathfrak{U}$, and so

$$
N_{\mathscr{B}^{2} \mathfrak{R}_{2}}(\mathfrak{S} \mathfrak{B}) \subseteq \mathbb{S}^{1} \Re_{1} \text {. }
$$

We conclude that $N_{\Theta^{i} \mathfrak{\vartheta}_{i}}(\mathfrak{S} \mathfrak{Z})$ is independent of $i, i=1,2$. Hence, $T$

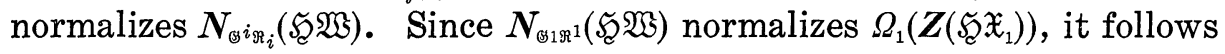

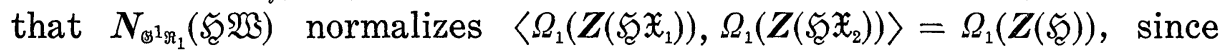

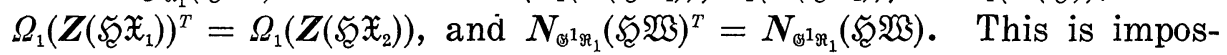
sible since $N\left(\Omega_{1}\left(Z\left(\mathfrak{S}_{\mathfrak{e}}\right)\right)\right)=\mathfrak{M}$. The proof is complete.

Hypothesis 15.2. $\mathfrak{T}$ is not a $S_{2}$-subgroup of $\mathbb{B}$.

Lemmas 15.16 through 15.26 are proved under Hypothesis 15.2.

Let $\mathbb{G}_{2}$ be a $S_{2}$-subgroup of $\mathbb{S}$ which contains $\mathfrak{I}$, and let $3=$ $\Omega_{1}\left(Z\left(\mathbb{S}_{2}\right)\right)$. Thus, $3 \subseteq \Omega_{1}(Z(\mathfrak{T})) \subseteq \mathfrak{B}$. By Theorem 15.1, we have $|\mathfrak{B}| \leqq 8$. Let $\Re_{1}=C_{\Re}(\mathfrak{B})$. Since $3 \subseteq \mathfrak{B}$, and $\mathfrak{S}_{2} \nsubseteq \mathfrak{M}$, it follows from Lemma 14.20 that $\Re_{1}$ is cyclic. Since $|\mathfrak{B}| \leqq 8$, we get $\left|\Re: \Re_{1}\right|=3$, and so $\Re^{\prime}=1, \Re=\Re_{1} \times \Re_{2}$, where $\left|\Re_{2}\right|=3, \Re_{2} \triangleleft N_{\mathfrak{m}}(\Re)$. Thus, $\boldsymbol{N}_{\mathfrak{m}}(\Re) / \boldsymbol{C}_{\mathfrak{m}}(\Re)$ is elementary of order 2 or 4 and $\Re \cong \mathfrak{M}^{\prime}$.

LEMMA 15.16. $\quad N_{\mathfrak{M}}(\Re) / C_{\mathfrak{m}}(\Re)$ is a four-group .

Proof. Suppose false, so that $\left|\boldsymbol{N}_{\mathfrak{M}}(\Re) / C_{\mathfrak{M}}(\Re)\right|=2$. Since $\Re \subseteq \mathfrak{M}^{\prime}$, and $C(\mathfrak{B}) \triangleleft \mathfrak{M}$, it follows that $C(\mathfrak{B})$ has a normal 3-complement. In this case, $\mathfrak{F}$ is a maximal element of ${U_{\mathfrak{R}}}_{(} \Re_{1} ; 2)$, and since $N(\mathfrak{C}) \subseteq \mathfrak{M}$, $\mathfrak{S}$ is a maximal element of $И\left(\Re_{1} ; 2\right)$. But $\Re_{1} \subseteq\left(\Re_{1} \mathfrak{I}\right)^{\prime}$, and by Lemma 
14.22, $N\left(\Omega_{1}\left(\Re_{1}\right)\right) \subseteq \mathfrak{M}$. Since a $S_{\{2,3\}}$-subgroup of $C(3)$ is a $Z$-group, it follows that $\Re_{1}$ centralizes some $S_{\{2,3\}}$,-subgroup of $C(3)$, and so $\mathscr{S}_{2} \Re_{1}$ is a group. Since $\mathfrak{K}$ is a maximal element of $N\left(\Re_{1} ; 2\right)$, and since $\mathfrak{S} \cong \mathbb{S}_{2}$, it follows that $\mathfrak{S}=\boldsymbol{O}_{2}\left(\mathbb{S}_{2} \Re_{1}\right)$. This gives $\mathbb{S}_{2} \subseteq N\left(\mathfrak{S}_{2}\right) \subseteq \mathfrak{M}$, which is false. The proof is complete.

\section{LEMMA 15.17. $|\mathfrak{B}|=4$.}

Proof. Suppose false. Since $\Re$ does not centralize $\mathfrak{B}$, we have $|\mathfrak{B}|>2$. By Theorem 15.1, we conclude that $|\mathfrak{B}|=8$. Hence, $\mathfrak{B}=$ $\mathfrak{B}^{0} \times \mathfrak{B}^{1}$, where $\mathfrak{B}^{0}=\mathfrak{C}_{\mathfrak{F}}(\mathfrak{R})$ is of order 2 , and $\mathfrak{B}^{1}=[\mathfrak{B}, \mathfrak{R}]$ is a fourgroup, normal in $\mathfrak{M}$. Thus, $\Omega_{1}(\boldsymbol{Z}(\mathfrak{T}))=\mathfrak{B}^{0} \times \mathfrak{B}_{0}$, where $\mathfrak{B}_{0}=\boldsymbol{Z}(\mathfrak{I}) \cap \mathfrak{B}^{1}$ is of order 2. Also, 3 is a subgroup of $\mathfrak{B}^{0} 3_{0}$ of order 2 distinct from $\mathfrak{B}^{0}$.

Let $\mathfrak{I}_{i}=C_{\mathfrak{I}}\left(\mathfrak{R}_{i} \mathfrak{S}_{\mathfrak{C}} / \mathfrak{S}\right)$, so that $\mathfrak{I} / \mathfrak{S}_{\mathfrak{C}}=\mathfrak{I}_{1} / \mathfrak{S} \times \mathfrak{I}_{2} / \mathfrak{S}_{\mathfrak{C}}$, and $\left|\mathfrak{I}_{i}: \mathfrak{S}_{\mathfrak{C}}\right|=2$. Thus, $\mathfrak{I}_{1}=\boldsymbol{O}_{2}\left(\mathfrak{T} \mathfrak{R}_{1}\right)$. Set $\mathfrak{M}_{0}=\mathfrak{T} \mathfrak{R}_{1}$. Since $\mathfrak{R}_{1} \subseteq \mathfrak{M}_{0}^{\prime}$ and $N\left(\Omega_{1}\left(\mathfrak{R}_{1}\right)\right) \subseteq \mathfrak{M}$, it follows that $\boldsymbol{C}(\mathbb{3})=\mathbb{S}_{2}(\boldsymbol{C}(\mathfrak{B}) \cap \mathfrak{M})$. Furthermore, $\boldsymbol{O}_{2}\left(\mathfrak{M}_{0}\right) \boldsymbol{C}_{\mathfrak{M}_{0}}(\mathfrak{B})=\mathfrak{M}_{0}$, since $\mathfrak{I}_{2}$ centralizes $\mathfrak{B}$. Let $\mathfrak{W}=\Omega_{1}\left(Z\left(\mathfrak{I}_{1}\right)\right)$. Then $\mathfrak{W} \cap \mathfrak{B}=\mathfrak{B}^{0} \mathfrak{Z}_{0}$. Let $(D)$ be a $S_{\{2,3\}}$-subgroup of $\mathfrak{M}$ permutable with $\Re$. Then $\mathfrak{R D}=\mathfrak{R} \times \mathfrak{D}$, since $\left(\mathcal{D}\right.$ is a $Z$-group, and $\mathfrak{R} \subseteq \mathfrak{M}^{\prime}$. Let $\mathfrak{M}_{1}=\mathfrak{I}_{1} \mathfrak{R}_{1} \mathfrak{D}$, so that $\mathfrak{M}_{1}$ is of index 2 in $\mathfrak{M}$. Let $\mathfrak{W}_{1}=\mathfrak{W}^{\mathfrak{M}_{1}}$. Since $\mathfrak{I}$ normalizes $\mathfrak{W}$, it follows that $\mathfrak{W}_{1} \triangleleft \mathfrak{M}$. Let $\mathfrak{E}_{1}=\boldsymbol{C}_{\mathfrak{M}_{1}}\left(\mathfrak{W}_{1}\right)$. Then $\mathfrak{M}_{1} / \mathfrak{S}_{1}$ has no non identity normal 2-subgroups, by Lemma 5.10. We argue that $\Re_{1}$ centralizes $\mathfrak{W}_{1}$. If $\boldsymbol{C}_{\mathfrak{M}}\left(\mathfrak{W}_{1}\right) \supset \boldsymbol{C}_{\mathfrak{M}_{1}}\left(\mathfrak{W}_{1}\right)$, then $\mathfrak{M} / \boldsymbol{C}_{\mathfrak{m}}\left(\mathfrak{W}_{1}\right) \cong \mathfrak{M}_{1} / \boldsymbol{C}_{\mathfrak{M}_{1}}\left(\mathfrak{W}_{1}\right)$, and so $\mathfrak{W}_{1}$ is 2-reducible in $\mathfrak{M}$, by Lemma 5.10. If $\mathfrak{W}_{1}$ is 2-reducible in $\mathfrak{M}$, then by definition of $\mathfrak{B}$, we get $\mathfrak{W}_{1} \subseteq \mathfrak{B} \subseteq C\left(\mathfrak{R}_{1}\right)$. Thus, we may assume that $\mathfrak{W}_{1}$ is not 2-reducible in $\mathfrak{M}$. Set $\mathfrak{E}_{1}=C_{\mathfrak{N}_{1}}\left(\mathfrak{W}_{1}\right)=\boldsymbol{C}_{\mathfrak{M}}\left(\mathfrak{W}_{1}\right)$, and let $\mathfrak{D}_{1} / \mathfrak{C}_{1}=\boldsymbol{O}_{2}\left(\mathfrak{M} / \mathfrak{C}_{1}\right)$. Since $\mathfrak{D}_{1} \cap \mathfrak{M}_{1}=\mathfrak{C}_{1}$, and $\left|\mathfrak{M}: \mathfrak{M}_{1}\right|=2$, if follows that $\left|\mathfrak{D}_{1}: \mathfrak{S}_{1}\right|=2$. Let $\mathfrak{D}_{2}=\mathfrak{I} \cap \mathfrak{D}_{1}$, so that $\mathfrak{I}=\mathfrak{D}_{2} \mathfrak{I}_{1}$. Since $\mathfrak{D}_{2} \nsubseteq \mathfrak{I}_{1}$, it follows that $\mathfrak{D}_{2}$ does not centralize $\mathfrak{R}_{1} \boldsymbol{O}_{3^{\prime}}(\mathfrak{M}) / \boldsymbol{O}_{3^{\prime}}(\mathfrak{M})$, and so $\mathfrak{R}_{1}$ is contained in the normal closure of $\mathfrak{D}_{2}$ in $\mathfrak{M}$. Since $\mathfrak{D}_{2} \subseteq \mathfrak{D}_{1} \triangleleft \mathfrak{M}$, we get $\mathscr{D}_{1} \supseteqq \Re_{1}$. Since $\left|\mathfrak{D}_{1}: \mathfrak{G}_{1}\right|=2$, we then get $\mathfrak{E}_{1} \supseteqq \Re_{1}$. Thus, in every case, $\Re_{1}$ centralizes $\mathfrak{W}_{1}$.

Set $\mathfrak{R}_{1}=\mathbb{G}_{2} \Re_{1}$, so that $\mathfrak{Q}_{1}$ is a group. Since $\mathfrak{I} \subset \mathbb{B}_{2}$, it follows that $\mathfrak{I}_{1} \subset \boldsymbol{O}_{2}\left(\mathbb{R}_{1}\right)$. Let $\mathfrak{I}^{1}=N_{\boldsymbol{o}_{2}\left(\Omega_{1}\right)}\left(\mathfrak{I}_{1}\right)$. Since $N\left(\Omega_{1}\left(\Re_{1}\right)\right) \subseteq \mathfrak{M}$, it follows that $\Re_{1}$ acts frobeniusly on $\mathfrak{I}^{1} / \mathfrak{I}_{1}$. Since $\mathfrak{W} \subseteq \mathfrak{W}_{1}$, and since $\mathfrak{I}^{1}$ normalizes $\mathfrak{W}=\Omega_{1}\left(\boldsymbol{Z}\left(\mathfrak{I}_{1}\right)\right.$ ), it follows that $\mathfrak{I}^{1}$ centralizes $\mathfrak{W}$. Hence, $\mathfrak{I}^{1} \subseteq C(\mathfrak{W}) \subseteq C\left(\mathfrak{B}^{0}\right) \subseteq \mathfrak{M}$, against $\mathfrak{I}_{1}=\boldsymbol{O}_{2}\left(\mathfrak{L}_{1}\right) \cap \mathfrak{M} \subset \mathfrak{I}^{1}$. The proof is complete.

We introduce the following additional notation: $\tilde{\Re}_{1}=\Omega_{1}\left(\Re_{1}\right)$, $\mathfrak{L}=\boldsymbol{C}(\mathbb{3})$. Let $\mathfrak{D}$ be a $S_{\{2,3\}}$-subgroup of $\mathfrak{M}$ which is permutable with $\mathfrak{R}$, and let $\Re=\mathfrak{D} \Re=\mathfrak{D} \times \mathfrak{R}$. Then $\Re_{1}=C_{\mathfrak{R}}(\mathfrak{Z})=\mathfrak{R}_{1} \times \mathfrak{D}$. Since $N\left(\widetilde{\Re}_{1}\right) \subseteq \mathfrak{M}$, it follows that $\mathbb{R}=\mathbb{S}_{2} \mathfrak{R}_{1}$. Now $\mathfrak{M}_{1}=\mathfrak{T}_{\mathfrak{R}_{1}}$ is of index 3 in $\mathfrak{M}$, and is thus a maximal subgroup of $\mathfrak{M}$. We argue that if $\mathfrak{X} \subseteq \mathfrak{M}$, and $\mathfrak{X}$ is a subgroup of $\mathfrak{M}$ which is permutable with $\mathfrak{S}_{2}$, then $\mathfrak{X} \subseteq \mathfrak{T} \mathfrak{R}_{1}$. If false, 
we get that $\mathscr{S}_{2}$ is permutable with $\left\langle\mathfrak{X}, \mathfrak{I} \mathscr{R}_{1}\right\rangle=\mathfrak{M}$, and so $\mathfrak{Q}^{*}=\mathfrak{M S S}_{2}$ is a group. Since $\mathfrak{B} \subseteq \mathbb{B}_{2}$, it follows that $\mathbb{S}_{2}$ contains a non identity normal subgroup of $\mathbb{2}^{*}$, and since $\mathbb{B}$ is an $N$-group, $\mathbb{2}^{*}$ is solvable. Since $\mathfrak{M} \in \mathscr{C l} \mathscr{S}(\mathfrak{S})$, we get $\mathfrak{R}^{*}=\mathfrak{M} \supseteqq \mathbb{S}_{2}$. This is false, and so $\mathfrak{T}_{1}$ contains every subgroup of $\mathfrak{M}$ which is permutable with $\mathfrak{S}_{2}$.

Lemma 15.18. $\quad \mathscr{G}_{2} \tilde{\Re}_{1} \in \mathscr{H}^{*}$, and $\mathfrak{R} \in \mathscr{M} \mathscr{S}(\mathbb{S})$.

Proof. Suppose $\mathbb{2}^{*}$ is a solvable subgroup of $\&$ which contains $\mathbb{S S}_{2} \tilde{\Re}_{1}$. Since $\tilde{\Re}_{1} \subseteq\left(\mathbb{S}_{2} \widetilde{R}_{1}\right)^{\prime}$, it follows that $\mathbb{2}^{*}=\mathbb{S}_{2}\left(\mathbb{2}^{*} \cap \mathfrak{M}\right)$. By the preceding argument, $\mathfrak{L}^{*} \cap \mathfrak{M} \subseteq \mathfrak{I} \mathfrak{R}_{1}$, and so $\mathfrak{2}^{*} \subseteq \mathfrak{L}$. The proof is complete.

Next, we show that

LEMMA 15.19. $\quad N\left(\Omega_{1}(\Re)\right) \leqq \mathfrak{M}$.

Proof. By Lemma 14.22, we have $C\left(\Omega_{1}(\Re)\right) \subseteq N\left(\tilde{\Re}_{1}\right) \subseteq \mathfrak{M}$. Let $\mathscr{R}$ be the set of all subgroups of $\Re$ of order 3 which have non trivial fixed points on $\mathfrak{F}$. Thus, $\Re_{1} \in \mathscr{R}$. Suppose $X \in N\left(\Omega_{1}(\Re)\right), X \notin \mathfrak{M}$. Then $\mathscr{R} \cap \mathscr{R}^{x}=\varnothing$, and so $|\mathscr{R}| \leqq 2$. Since $\Re$ acts faithfully on $\mathfrak{K}$, we conclude that $|\mathscr{R}|=2$. Since $\mathscr{R}$ is normalized by $N_{\mathfrak{m}}(\Re)$, it follows that $\mathscr{R}=\left\{\tilde{\Re}_{1}, \Re_{2}\right\}$. Since $\tilde{\Re}_{1}$ and $\Re_{2}$ are the only subgroups of $\Re$ of order 3 which are normalized by $N_{\Re}(\Re)$, and since we may assume without loss of generality that $X$ normalizes $N_{\mathfrak{n}}\left(\Omega_{1}(\Re)\right)$, it follows that $\mathscr{R}=\mathscr{R}^{x}$. This contradiction completes the proof.

LeMma 15.20. If $\mathfrak{S}$ is a 3-subgroup of (S) which contains $\Re_{2}$, then $N(\Im) \subseteq N\left(\Re_{2}\right)$.

Proof. We may choose $C$ in $C\left(\Re_{2}\right)$ such that $\mathfrak{S}^{C} \subseteq \Re$. Thus, we may assume at the outset that $\subseteq \subseteq \Re$. We may also assume that $\mathfrak{S} \supset \Re_{2}$. Hence, $\mathfrak{S}=\Re_{2} \times\left(\mathfrak{S} \cap \Re_{1}\right)$, and so $\mathfrak{S} \supseteqq \Omega_{1}(\Re)$, whence $\Omega_{1}(\mathfrak{S})=$ $\Omega_{1}(\Re)$. Since $\Re_{2} \triangleleft N_{\mathfrak{M}}(\Re)$ by construction, this lemma follows from Lemma 15.19.

LeMma 15.21. $\mathfrak{I}$ is a $S_{2}$-subgroup of every solvable subgroup of (B) which contains $\mathfrak{T} \Re_{2}$.

Proof. Suppose false, and $\sqrt{S}$ is a solvable subgroup of $\$ 3$ which is minimal subject to

(a) $\mathfrak{I}$ is not a $S_{2}$-subgroup of $\mathfrak{S}$.

(b) $\mathfrak{T} \Re_{2} \subset \mathfrak{S}$.

Then $\subseteq$ is a 2,3-group. Let $\left\{\mathfrak{S}_{2}, \mathfrak{S}_{3}\right\}$ be a Sylow system of $\mathfrak{S}$ with $\mathfrak{I} \subset \mathfrak{S}_{2}, \mathfrak{R}_{2} \subseteq \mathfrak{S}_{3}$. Let $\tilde{\Re}=\boldsymbol{O}_{2}(\mathfrak{S})$, so that $\subseteq=\tilde{\Re} \cdot \boldsymbol{N}_{\widetilde{\subseteq}}\left(\mathfrak{S}_{3}\right)$. By Lemma 


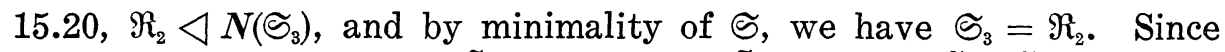
$\Re_{2} \subseteq\left(\mathfrak{T} \mathfrak{R}_{2}\right)^{\prime}$, we have $\mathfrak{S}_{2}=\tilde{\mathscr{R}} \mathfrak{T}$, and so $\left|\mathfrak{S}_{2}: \tilde{\mathfrak{R}}\right|=2$, and $\tilde{\mathscr{R}} \supset \widetilde{\Re} \cap \mathfrak{M}=\mathfrak{R}_{0}$, say. Now $\Re_{0}=\mathfrak{I}_{2}$ satisfies $\left|\mathfrak{T}: \mathfrak{T}_{2}\right|=2=\left|\mathfrak{T}_{2}: \mathfrak{K}\right|$. Also $\mathfrak{B} \cong \Omega_{1}\left(Z\left(\mathfrak{I}_{2}\right)\right)$. Since $Z(\mathfrak{I})$ is cyclic, it follows that $\mathfrak{B}=\Omega_{1}\left(Z\left(\mathfrak{I}_{2}\right)\right)$. Since $\mathfrak{M}=N(\mathfrak{B})$, $\mathfrak{I}$ is forced to be a $S_{2}$-subgroup of $\mathfrak{S}$, since $N\left(\mathfrak{R}_{0}\right)=N\left(\mathfrak{I}_{2}\right) \subseteq \mathfrak{M}$. The proof is complete.

LEMMA 15.22. $\left|\mathbb{B S}_{2}: \mathfrak{I}\right|$ is a square, and $\left|\mathbb{S}_{2}: \mathfrak{T}\right| \geqq 4$.

Proof. $\mathbb{R}_{1}=\mathbb{S}_{2} \tilde{\Re}_{1}$ is a group, and $\mathbb{R}_{2}=\boldsymbol{O}_{2}\left(\mathbb{S}_{2} \Re_{1}\right)$ is of index 2 in $\mathbb{S}_{2}$, and $\mathbb{S}_{2}=\mathfrak{R}_{2} \mathfrak{T}$. Hence, $\left|\mathbb{S}_{2}: \mathfrak{T}\right|=\mathfrak{R}_{2}: \mathfrak{I}_{1} \mid$, since $\mathfrak{I}_{1}=\mathfrak{T} \cap \boldsymbol{O}_{2}\left(\mathfrak{R}_{1}\right)$. Let $\mathfrak{I}_{1}=\mathfrak{W}_{1} \subset \mathfrak{W}_{2} \subset \ldots \subset \mathfrak{W}_{s}=\mathfrak{R}_{2}$ be a chain of $\tilde{\mathfrak{R}}_{1}$-admissible subgroups of $\mathfrak{R}_{2}$ such that $\tilde{\mathfrak{R}}_{1}$ acts irreducibly on $\mathfrak{W}_{i+1} / \mathfrak{W}_{i}, i=1, \cdots, s-1$. Since $N\left(\tilde{\Re}_{1}\right) \cong \mathfrak{M}$, it follows that each such quotient is a four-group. The lemma follows.

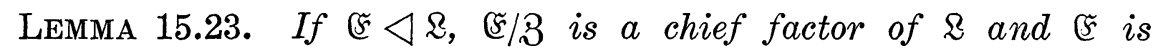
elementary abelian, then $\left[\subseteq \subseteq \boldsymbol{O}_{2}(\mathfrak{M})\right.$.

Proof. Since $\mathfrak{B} \in \boldsymbol{h}_{\mathfrak{\&}}\left(\Re_{1} ; 2\right)$, we have $\mathfrak{B} \subseteq \boldsymbol{O}_{2}(\mathfrak{L})$, and so $[\mathfrak{F}, \mathfrak{B}] \subseteq$ $\mathfrak{B} \subseteq \mathfrak{B}$. Hence, $\mathfrak{F} \subseteq N(\mathfrak{B})=\mathfrak{M}$.

Let $\mathfrak{F}_{1}=\mathbb{F} \cap \boldsymbol{O}_{2}(\mathfrak{M})$. Thus, $\mathbb{F} / \mathbb{F}_{1}$ acts faithfully on $\boldsymbol{O}_{2,2}(\mathfrak{M}) / \boldsymbol{O}_{2}(\mathfrak{M})$. Since $\Re_{1}$ is of index 3 in $\Re$, and since $\Re \cap \boldsymbol{O}_{2,2^{\prime}}(\mathfrak{M})$ covers $\boldsymbol{O}_{2,2^{2}}(\mathfrak{M}) / \boldsymbol{O}_{2}(\mathfrak{M})$, it follows that $\mathfrak{S} / \mathfrak{G}_{1}$ has order at most 2 . Suppose by way of con-

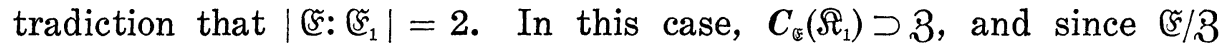
is a chief factor of $\mathbb{R}$, it follows that $\mathbb{F}$ is a four-group, $\mathfrak{F}_{1}=3$.

Choose $E \in \mathbb{F}-3$. Then we can choose an element $F$ of $\mathfrak{M}$ of

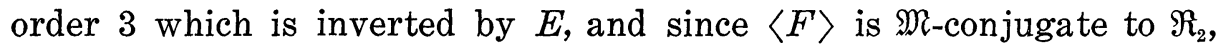

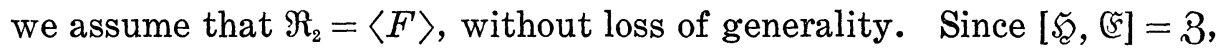
it follows that $\mathfrak{F}=\mathfrak{B} \times C_{\mathfrak{F}}\left(\Re_{2}\right)$. Since $\Re_{2} \triangleleft N_{\mathfrak{n}}(\Re)$, it follows that $\boldsymbol{C}_{\mathfrak{S}}\left(\Re_{2}\right) \triangleleft \mathfrak{T}$. Since $\boldsymbol{Z}(\mathfrak{T})$ is cyclic, and $\Omega_{1}(\boldsymbol{Z}(\mathfrak{T})) \subseteq \mathfrak{B}$, it follows that $\boldsymbol{Z}(\mathfrak{T}) \cap \boldsymbol{C}_{\mathfrak{y}}\left(\Re_{2}\right)=1$, whence, $\boldsymbol{C}_{\mathfrak{5}}\left(\Re_{2}\right)=1$. This means that $\mathfrak{S}_{\mathfrak{F}}=\mathfrak{B}$ is a four-group, which is false, since $\Re$ acts faithfully on $\mathfrak{F}$. The proof is complete.

Since $\mathbb{R} \in \mathscr{C} \mathscr{S}(\mathbb{S})$, Theorems $13.5,13.6,13.7$ imply that $\mathbb{R}$ contains a non cyclic normal abelian subgroup. So $\mathbb{R}$ contains an elementary abelian normal 2-subgroup \& such that $\mathbb{F} / \mathbb{3}$ is a chief factor of $\mathbb{L}$. We retain this notation.

LEMma 15.24. If $\mathfrak{F}_{0}$ is a hyperplane of $\mathfrak{F}$, then $C\left(\mathfrak{F}_{0}\right) \subseteq \mathrm{I}$.

Proof. Suppose false. Then $3 \nsubseteq \mathfrak{F}_{0}$, and so $\mathbb{F}=\mathfrak{F}_{0} \times 3$. Let $\mathfrak{E}_{1}=\boldsymbol{C}(\mathfrak{E}), \quad \mathfrak{D}_{1} / \mathfrak{S}_{1}=\boldsymbol{O}_{2}\left(\mathbb{R} / \mathfrak{S}_{1}\right)$. Since $3=\Omega_{1}\left(\boldsymbol{Z}\left(\mathbb{S}_{2}\right)\right)$, we have $\mathfrak{E}_{1} \cap \mathbb{Z}$. Suppose $\mathfrak{D}_{1}=\mathfrak{E}_{1}$. In this case, $\boldsymbol{O}_{2^{\prime}}\left(\mathbb{R} / \mathfrak{S}_{1}\right)=\mathfrak{D}_{2} / \mathfrak{S}_{1} \neq 1$. Let $\Re_{2}$ be a 
$S_{2}$-subgroup of $\mathfrak{D}_{2}$. Thus, since $\mathbb{5} / 3$ is a chief factor of $\mathbb{2}$, it follows that $3=C_{\mathbb{E}}\left(\mathfrak{D}_{2}\right)$, and so $\mathbb{F}=3 \times\left[\mathfrak{D}_{2}, \mathfrak{F}\right]$, whence $\left[\mathfrak{F}, \mathfrak{D}_{2}\right] \triangleleft \mathbb{R}$. This is false, since $3=\Omega_{1}\left(Z\left(\mathscr{S}_{2}\right)\right)$. Hence, $\mathfrak{D}_{1} / \mathfrak{C}_{1} \neq 1$. Since $\mathbb{S} / 3$ is a chief

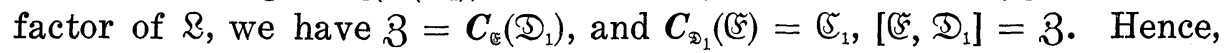
$\left[5 / 3\right.$ and $\mathfrak{D}_{1} / \mathbb{S}_{1}$ are in duality, and so $\mathfrak{D}_{1}$ permutes transitively the hyperplanes of 5 which do not contain 3. Thus, we assume without loss of generality that $\Re_{1} \subseteq N\left(\mho_{0}\right)$.

First, suppose $\widetilde{R}_{1}$ centralizes $\mathfrak{F}$. Since $\mathbb{F} \triangleleft \mathscr{S}_{2}$, there is a fourgroup $\mathfrak{U} \triangleleft \mathfrak{S}_{2}, \mathfrak{U} \leqq \mathfrak{F}_{\text {. }}$ Let $\mathfrak{U}_{0}=\mathfrak{F}_{0} \cap \mathfrak{U}$, so that $\left|\mathfrak{U}_{0}\right|=2$. Let $\widetilde{\mathfrak{S}}_{2}=\boldsymbol{C}_{\mathbb{\mho}_{2}}\left(\mathfrak{U}_{0}\right)$ so that $\widetilde{\mathbb{S}}_{2}$ is of index 2 in $\mathbb{S}_{2}$, and $\mathfrak{U}=\Omega_{1}\left(Z\left(\widetilde{G}_{2}\right)\right)$. Since $\mathbb{S}_{2} \widetilde{\Re}_{1} \subseteq N(\mathfrak{U})$, it follows that $N(\mathfrak{U}) \cong \mathfrak{R}$, and so $\widetilde{\mathbb{S}}_{2}$ is a $S_{2}$-subgroup of $C\left(\mathfrak{U}_{0}\right)$.

Suppose that $\boldsymbol{C}\left(\mathfrak{u}_{0}\right)$ has a normal 3-complement $\widetilde{\mathbb{S}}$. In this case, $\tilde{\Re}_{1}$ normalizes $\widetilde{\mathscr{G}}_{2}$, and if $\mathfrak{W}$ is any non indentity characteristic subgroup of $\widetilde{\mathbb{S}}_{2}$, it follows that $\mathbb{S}_{2} \Re_{1} \subseteq N(\mathfrak{W})$, and so $N(\mathfrak{W}) \subseteq \mathbb{R}$, by Lemma 15.18. This in turn implies that $\boldsymbol{O}_{3^{\prime}}\left(\boldsymbol{C}\left(\mathfrak{H}_{0}\right)\right)=\widetilde{\mathbb{C}} \subseteq \mathbb{R}$, since $\widetilde{\mathbb{C}}=(\widetilde{\mathbb{C}} \cap$ $\boldsymbol{C}\left(\boldsymbol{Z}\left(\widetilde{\mathfrak{S}}_{2}\right)\right) \cdot\left(\widetilde{\mathfrak{S}} \cap \boldsymbol{N}\left(\boldsymbol{J}\left(\widetilde{\mathfrak{S}}_{2}\right)\right)\right.$. Thus, if $\mathfrak{D}_{3}$ is a $S_{3}$-subgroup of $\boldsymbol{C}\left(\mathfrak{U}_{0}\right)$ which contains $\widetilde{R}_{1}$, then $\mathfrak{D}_{3} \nsubseteq \mathbb{L}$. This in turn implies that $\mathfrak{D}_{3} \subseteq \mathfrak{M}$, $\left|\mathfrak{D}_{3}: \mathfrak{D}_{3} \cap \mathfrak{L}\right|=3$, and so $3^{\Phi_{3}}=\mathfrak{B}=3^{C\left(\mathfrak{u}_{0}\right)}$. Hence, $C\left(\mathfrak{H}_{0}\right) \subseteq \mathfrak{M}$, and so $|\mathfrak{I}| \geqq\left|\widetilde{\mathfrak{S}}_{2}\right|=\left|\mathbb{B}_{2}\right| / 2$, against Lemma 15.22. We conclude that $\boldsymbol{C}\left(\mathfrak{u}_{0}\right)$ has no normal 3-complement.

Suppose $\subseteq$ is an elementary subgroup of $C\left(\mathfrak{H}_{0}\right)$ of order 9 with $\widetilde{\mathfrak{R}}_{1} \subseteq \mathfrak{S}$. We assume without loss of generality that $\mathfrak{S}_{2}=\tilde{\mathfrak{\Omega}}$ is a group. Let $\tilde{S}_{0}=\boldsymbol{O}_{2}(\tilde{\mathfrak{R}})$. Since $\boldsymbol{C}_{\mathfrak{F}_{0}}\left(\widetilde{\mathfrak{R}}_{1}\right) \neq 1$, and $\boldsymbol{C}_{\mathfrak{5}}\left(\widetilde{\mathfrak{R}}_{1}\right) \neq 1$, $\mathfrak{S}_{0}$ is conjugate to a subgroup of $\mathfrak{F}_{2}$. If $\mathscr{S}_{0}$ is conjugate to $\mathfrak{F}$, we get $|\mathfrak{T}| \geqq$ $\left|\widetilde{\mathscr{S}}_{2}\right|$, which is false. Hence, $\mathfrak{H}_{0}$ is conjugate to a proper subgroup of $\mathfrak{S}$. Since $\left|\widetilde{\mathscr{S}}_{2}: \mathfrak{S}_{0}\right| \leqq 4$, it follows that $\left|\mathscr{S}_{2}: \mathfrak{F}\right| \leqq 4$, against $|\mathfrak{I}: \mathfrak{S}|=4$. This contradiction shows that $S_{3}$-subgroups of $C\left(\mathfrak{U}_{0}\right)$ are cyclic.

Since $\boldsymbol{C}\left(\mathfrak{U}_{0}\right)$ has no normal 3-complement, it follows that $\widetilde{\mathfrak{R}}_{1} \subseteq \boldsymbol{C}\left(\mathfrak{H}_{0}\right)^{\prime}$. Since a $S_{\{2,3\}^{\prime}}$-subgroup of $C\left(\mathfrak{U}_{0}\right)$ is a $Z$-group, it follows that $C\left(\mathfrak{U}_{0}\right) \cap$ $C \widetilde{\Re}_{1}$ ) contains a $S_{2^{\prime}}$-subgroup of $\boldsymbol{C}\left(\mathfrak{H}_{0}\right)$. Since $\mathfrak{D} \subseteq \mathbb{R} \cap \mathfrak{M}$, it follows that if $\subseteq$ is a $S_{3}$-subgroup of $C\left(\mathfrak{U}_{0}\right)$ which contains $\widetilde{\mathfrak{R}}_{1}$, then $\subseteq \subseteq \mathfrak{M}$, $\mathfrak{S} \nsubseteq \mathfrak{L}$. Hence, $3^{\mathfrak{S}}=\mathfrak{3}^{c\left(\mathfrak{u}_{0}\right)}=\mathfrak{B}$, so $\widetilde{\mathbb{S}}_{2} \subseteq N(\mathfrak{B}) \subseteq \mathfrak{M}$, whence $|\mathfrak{T}| \geqq$ $\left|\mathscr{S}_{2}\right|=\left|\mathscr{S}_{2}\right| / 2$, which violates Lemma 15.22 .

We may now assume that $\left[\widetilde{\Re}_{1}, \mathfrak{s}\right] \neq 1$. In this case, $1 \subset\left[\mathfrak{F}_{0}, \widetilde{\Re}_{1}\right] \subseteq \mathfrak{F}_{0}$, and so $\left[\widetilde{R}_{1}, \mathfrak{F}_{0}\right]$ contains a 4-group $\mathfrak{U}$ which admits $\widetilde{\mathfrak{R}}_{1}$ and such that $\mathfrak{U} 3 \triangleleft \mathfrak{S}_{2}$. Since $C\left(\mathfrak{F}_{0}\right) \varsubsetneqq \mathbb{R}$, so also $\mathfrak{N}=N(\mathfrak{U}) \nsubseteq \mathfrak{R}$. Let $\mathfrak{R}_{2}=N_{\Theta_{2}}(\mathfrak{l} \mathfrak{l})$, so that $\Re_{2}$ is of index 4 in $\mathbb{S}_{2}$.

Since $\widetilde{\Re}_{1}$ does not centralize $\mathfrak{F}$, and since $\mathbb{F} / 3$ is a chief factor of R, which $\widetilde{\mathfrak{R}}_{1} \boldsymbol{O}_{2}(\mathbb{R}) \triangleleft \mathbb{R}$, it follows that $\widetilde{\mathfrak{R}}_{1} \subseteq\left(\mathfrak{\Re}_{2} \widetilde{\Re}_{1}\right)^{\prime}$, and so $N(\mathfrak{l})=$ $\mathfrak{R}_{2}^{*}\left(\boldsymbol{N}(\mathfrak{U}) \cap C\left(\widetilde{\mathfrak{R}}_{1}\right)\right)=\mathfrak{N}_{2}^{*}(N(\mathfrak{I}) \cap \mathfrak{M})$, where $\mathfrak{R}_{2}^{*}$ is a $S_{2}$-subgroup of $N(\mathfrak{U})$ which contains $\mathfrak{R}_{2}$. Since $\widetilde{\mathfrak{R}}_{1}$ does not centralize $\mathfrak{U}$, it follows that if S is a $S_{3}$-subgroup of $N(\mathfrak{l})$ which contains $\widetilde{\Re}_{1}$, then $\widetilde{\Re}_{1}$ is a direct factor of $\mathfrak{S}$. 
Suppose $\subseteq \supset \widetilde{\Re}_{1}$. In this case, we get $|\mathfrak{S}|=9$, since $\widetilde{\Re}_{1} \triangleleft N(\Re)$, and $\left|\Re_{2}\right|=3$. We then get that $\mathfrak{R}^{*}=\mathfrak{R}_{2}^{*} \subseteq$ is a group. Furthermore, $\boldsymbol{C}_{\mathfrak{x}}\left(\widetilde{\Re}_{1}\right)$ is a $S_{2}$-subgroup of $\boldsymbol{C}\left(\widetilde{\Re}_{1}\right)$ and since $\boldsymbol{C}_{\mathfrak{x}}\left(\widetilde{\Re}_{1}\right)$ normalizes $\mathfrak{1} 3, \boldsymbol{C}_{\mathfrak{x}}\left(\widetilde{\Re}_{1}\right)$ also normalizes $\left[\mathfrak{u} 3, \widetilde{\Re}_{1}\right]=\mathfrak{U}$. Thus, $\mathfrak{\Re}_{2}$ contains a $S_{2}$-subgroup of $C\left(\widetilde{\Re}_{1}\right)$. This implies that either $\mathfrak{N}_{2}^{*}=\mathfrak{N}_{2}$ or $\mathfrak{N}_{2}^{*}$ is a $S_{2}$-subgroup of $\mathbb{B}$. Since $3 \subseteq \mathfrak{R}_{2}^{*}$, it follows that $\boldsymbol{O}_{2}\left(\mathfrak{R}^{*}\right)$ is conjugate to a subgroup of $\mathfrak{S}_{\text {. If }}$ $\boldsymbol{O}_{2}\left(\mathfrak{R}^{*}\right)$ is conjugate to a proper subgroup of $\mathfrak{S}$, we get $|\mathfrak{S}| \geqq$ $2\left|\boldsymbol{O}_{2}\left(\mathfrak{R}^{*}\right)\right| \geqq\left|\mathfrak{R}_{2}^{*}\right| / 2 \geqq\left|\mathscr{S}_{2}\right| / 8$, whence $|\mathfrak{I}| \geqq\left|\mathscr{S}_{2}\right| / 2$, against Lemma 15.22. Hence, $\boldsymbol{O}_{2}\left(\mathfrak{R}^{*}\right)$ is conjugate to $\mathfrak{S}$, whence $\mathfrak{N}_{2}=\mathfrak{N}_{2}^{*}$, and $\left|\mathbb{S}_{2}: \mathfrak{I}\right|=4$. This in turn implies that $\mathfrak{U} \sim \mathfrak{B}$, since $\mathfrak{U}=\Omega_{1}\left(\boldsymbol{Z}\left(\mathfrak{N}_{2}\right)\right)^{\mathfrak{5}}$, and $\mathfrak{B}=$ $\Omega_{1}(\boldsymbol{Z}(\mathfrak{T}))^{\Omega_{1}(\mathfrak{x})}$. Let $\mathfrak{S}_{1}=\boldsymbol{C}_{\mathfrak{S}}(\mathfrak{l})$, so that $\left|\mathfrak{S}_{1}\right|=3$, and $\widetilde{S}_{1} \sim \widetilde{\mathfrak{R}}_{1}$ This implies that $\widetilde{S}$ contains more than one conjugate of $\widetilde{\Re}_{1}$, which is false, since $\widetilde{\Re}_{1} \triangleleft N(\Re)$. So $\widetilde{\Re}_{1}$ is a $S_{3}$-subgroup of $N(\mathfrak{H})$.

Since $\mathfrak{R}_{1} \subseteq \mathfrak{N}^{\prime}$, it follows that $\mathfrak{R}=\mathfrak{R}_{2}^{*}(\mathfrak{M} \cap \mathfrak{R})$, and since $\widetilde{\Re}_{1}$ is a $S_{3}$-subgroup of $\mathfrak{R}, \mathfrak{M} \cap \mathfrak{R} \subseteq \mathbb{R}$. Thus, $\mathfrak{R}_{2}^{*} \nsubseteq \mathfrak{R}$, so that $\mathfrak{R}_{2} \cap \mathfrak{R}_{2}^{*}$. Since $\boldsymbol{C}_{\mathfrak{x}}\left(\widetilde{\Re}_{1}\right)$ is a $S_{2}$-subgroup of $\boldsymbol{C}\left(\widetilde{\mathfrak{R}}_{1}\right)$, and since $\boldsymbol{C}_{\mathfrak{x}}\left(\widetilde{\mathfrak{R}}_{1}\right)$ normalizes $\mathfrak{U}$, it follows that $\mathfrak{R}_{2}^{*}$ is a $S_{2}$-subgroup of $\$$.

Let $\mathfrak{2}^{*}=\mathfrak{R}_{2}^{*} \widetilde{\mathfrak{R}}_{1}, \quad \mathfrak{R}_{2}^{*}=\boldsymbol{O}_{2}\left(\mathbb{2}^{*}\right), \quad \mathfrak{\Omega}_{2}=\boldsymbol{O}_{2}\left(\mathfrak{N}_{2} \widetilde{\Re}_{1}\right)$. Thus, $\mathfrak{\Omega}_{2} \triangleleft \mathbb{\Omega}_{2}^{*}$ and $\Omega_{2}^{*} / \Omega_{2}$ is a four-group. On the other hand, since $\left|\boldsymbol{O}_{2}\left(\mathbb{S}_{2} \widetilde{\Re}_{1}\right): \Omega_{2}\right|=4$, and since $\mathbb{R}_{2}$ contains a $S_{2}$-subgroup of $C\left(\widetilde{\Re}_{1}\right)$, it follows that $\mathbb{S}_{2} \triangleleft \boldsymbol{O}_{2}\left(\mathscr{S}_{2} \widetilde{\Re}_{1}\right)$. Since $\mathbb{S}_{2} \widetilde{\Re}_{1}=\boldsymbol{O}_{2}\left(\mathbb{S}_{2} \widetilde{\Re}_{1}\right) \cdot \mathfrak{R}_{2} \widetilde{\Re}_{1}$, it follows that $\mathbb{G}_{2} \tilde{\Re_{1}} \cong N\left(\mathbb{R}_{2}\right)$, and so $N\left(\mathfrak{R}_{2}\right) \subseteq \mathfrak{R}$. Thus, $\mathfrak{R}_{2}^{*} \subseteq \mathfrak{L}$. Since $\mathfrak{R}_{2}^{*}=\mathfrak{R}_{2}^{*}\left(\mathfrak{R}_{2} \widetilde{\mathfrak{R}}_{1}\right)$, it follows that $\boldsymbol{N}(\mathfrak{I}) \subseteq \mathfrak{R}$. The proof is complete.

LEMMA 15.25. $\quad N(\mathfrak{P}) \leqq \mathfrak{M}$ for every subgroup $\mathfrak{P}$ of $\mathfrak{M}$ of prime order $p$, and every $p \geqq 5$.

Proof. We may assume that $\Re \subseteq C(\mathfrak{P})$. Thus, $\mathfrak{B} \Re \subseteq N(\mathfrak{P})=\mathfrak{R}$, say. Since $S_{p}$-subgroups of $\mathfrak{M}$ are cyclic, we have $\mathfrak{R} \subseteq \mathfrak{R}^{\prime}$. Also, $\mathfrak{B} \subseteq \boldsymbol{O}_{2}(\mathfrak{L})$, since $\mathfrak{\Re}_{1}$ centralizes $\mathfrak{B}$. Since $\mathfrak{B}^{\sharp}$ is permuted transitively by $\mathfrak{M}$, it follows that $\mathfrak{B} \leqq \boldsymbol{O}_{2}(C(V))$ for all involutions $V$ of $\mathfrak{B}$. This implies that $\boldsymbol{O}_{2}(\mathfrak{R}) \neq 1$. Hence, $\Re$ centralizes some $S_{\{2,3\}}$,-subgroup of $\mathfrak{R}$, and so $\mathfrak{R}=\mathfrak{R}_{2}(\mathfrak{M} \cap \mathfrak{N})$, where $\mathfrak{R}_{2}$ is a $S_{2}$-subgroup of $\mathfrak{R}$ permutable with $\Re$. Since $C(\Re) \subseteq \mathfrak{M}$, and since there is $C$ in $C(\Re)$ such that $\boldsymbol{O}_{2}\left(\mathfrak{N} \mathfrak{R}_{2}\right)^{C} \subseteq \mathfrak{F}$, and since $\mathfrak{N}_{2} \Re=\boldsymbol{O}_{2}\left(\mathfrak{R}_{2} \Re\right) \cdot \boldsymbol{N}_{\mathfrak{K}_{2} \Re}(\mathfrak{R})$, it follows that $\mathfrak{R}_{2} \subseteq \mathfrak{M}$. The proof is complete.

LEMMA 15.26. There is $G$ in (s) such that $\mathfrak{F}^{G} \subseteq \mathfrak{I}, \mathfrak{F}^{G} \nsubseteq \mathfrak{I}_{2}=$ $\boldsymbol{O}_{2}\left(\mathfrak{T} \Re_{2}\right)$.

Proof. Let $\mathfrak{X}=V\left(\operatorname{ccl}_{\mathscr{\Theta}}(\mathfrak{S}) ; \mathfrak{I}\right)$. By Lemma 15.23 , we have $\mathfrak{\Xi} \leqq \mathfrak{X}$. If $\mathfrak{X} \subseteq \mathfrak{I}_{2}$, then $N(\mathfrak{X}) \supseteqq \mathfrak{T}_{2}$, and $\mathfrak{I}$ is not a $S_{2}$-subgroup of $N(\mathfrak{X})$, against Lemma 15.21. The proof is complete. 
Choose $G$ in $\mathbb{S}$ such that $\mathfrak{F}=\mathfrak{F}^{G} \subseteq \mathfrak{T}, \mathfrak{F} \nsubseteq \mathfrak{I}_{2}$. Let $\mathfrak{F}_{1}=\mathfrak{F} \cap \mathfrak{I}_{2}$, so that $\mathfrak{F}_{1}$ is a hyperplane of $\mathfrak{F}$. Then $\mathfrak{B} \subseteq C\left(\mathfrak{F}_{1}\right) \subseteq N(\mathfrak{F})$, and so $3=[\mathfrak{B}, \mathfrak{F}] \leqq \widetilde{F}$. It is important to show that $3 \neq 3^{G}$. Suppose $3=3^{G}$. In this case, $\mathfrak{F}=\mathfrak{F}$. By Lemma 15.23, $\mathfrak{F} \subseteq O_{2}(\mathfrak{M})$, against $₫ \varsubsetneqq \mathfrak{I}_{2}$. So $3 \neq 3^{G}$. Choose $V \in \mathfrak{B}-3$. Thus, $V$ normalizes $\widetilde{F}$ and $V$ is not 1 on $\widetilde{F} / 3^{a}$. More precisely, $[\mathfrak{F}, \mathfrak{B}]=3 \neq 3^{a}$. Set $2^{*}=\mathbb{2}^{a}$, and $3^{*}=3^{a}$. Let $\mathfrak{X}=C_{2^{*}}\left(\mathfrak{F} / \mathfrak{Z}^{*}\right)$, and let $\mathfrak{Y}=\mathfrak{2}^{*} \mathfrak{X}$. For each subset $\subseteq$ of $\mathfrak{2}^{*}$, let $\bar{\Im}$ be the image of $\subseteq$ in $\mathfrak{Y}$. Then $\bar{V} \neq 1$, and $\bar{V}$ is a transvection on $\mathfrak{F} / 3^{*}$. Let $\mathfrak{B}=F(\overline{\mathbb{Q}} *)$, so that $\bar{V}$ acts faithfully on $\mathfrak{B}$. Since a $S_{1^{\prime}}$-subgroup of $\mathfrak{R}^{*}$ is a $Z$-group, it follows that every subgroup of $\mathfrak{B}$ is characteristic in $\overline{\mathfrak{L}}^{*}$. Since $\bar{V}$ is a transvection on $\widetilde{F} / \mathbb{Z}^{*}$, it follows that $[\mathfrak{B}, \bar{V}]=\mathfrak{B}_{0}$ is of order 3 , and that $\left[\mathfrak{F} / \mathfrak{Z}^{*}, \mathfrak{B}_{0}\right]$ is a four-group. Since $\left[\mathfrak{F} / \mathfrak{Z}^{*}, \mathfrak{B}_{0}\right]$ admits $\bar{\Omega}^{*}$, it follows that $|\widetilde{F}|=8$. Hence, $|\mathfrak{F}|=8$, ond $\mathbb{L}$ permutes transitively the 6 involutions of $F-3$. Since $3 \neq 3^{G}$, it follows that all involutions of $(5$ are fused to $Z$ in (\$), where $3=\langle Z\rangle$.

Let $\Omega_{2}=C_{\mathbb{R}_{1}}(\mathfrak{F})$, so that $\left|\Re_{1}: \Omega_{2}\right|=3$. Choose $\mathfrak{U} \triangleleft \mathfrak{S}_{2}$, $\mathfrak{U} \leqq \mathfrak{F}$, $\mathfrak{U}$ a four-group, and let $\widetilde{\mathscr{S}}_{2}=\boldsymbol{C}_{\mathbb{S}_{2}}(\mathfrak{H})$, so that $\widetilde{\mathscr{S}}_{2}$ is of index a in $\mathbb{S}_{2}$. Also, since $\boldsymbol{Z}\left(\mathbb{S}_{2}\right)$ is cyclic, we have $\mathfrak{U}=\Omega_{1}\left(\boldsymbol{Z}\left(\widetilde{\mathfrak{S}}_{2}\right)\right)$, and since all involutions of $\mathfrak{U}$ are fused to 3 , we get $N(\mathfrak{l}) / C(\mathfrak{l}) \cong$ Aut $(\mathfrak{U})$. Let $\mathfrak{R}=N(\mathfrak{U}) \supseteqq$

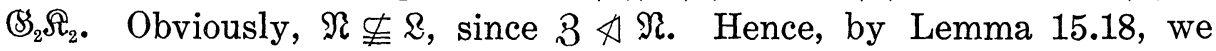
have $\widetilde{\Re}_{1} \nsubseteq N(\mathfrak{l})$. This implies that $\widetilde{\Re}_{1}=\Re_{1}$, since $\Re_{2}$ is of index 3 in $\Omega_{1}$. Let $\Re^{1}$ be a $S_{2}$-subgroup of $N(\mathfrak{H})$ which contains $\Re_{2}$. Since $\Omega_{2}$ is now a 3 -group, it follows that if $\Omega_{2} \neq 1$, then $N\left(\Omega_{2}\right) \subseteq \mathfrak{M}$. Suppose $\Omega_{2} \neq 1$. In this case, we get $\Re^{1} \subseteq \mathfrak{M}$, and by a previous argument, we then get $\Re^{1} \subseteq \mathfrak{T} \Re_{1}$, whence $\Re^{1} \subseteq \mathfrak{L}$. This is false, since $N(\mathfrak{U})$ is transitive on $\mathfrak{U}^{\sharp}$. We conclude that $\Re_{2}=1$. This means that $\mathfrak{M}=\mathfrak{T} \Re$ and $|\Re|=9$.

Let $\mathscr{F}_{0}=\left\{\mathfrak{F} \mid \mathfrak{F} \in \operatorname{ccl}_{\mathfrak{G}}(\mathfrak{F}), \mathfrak{F} \subseteq \mathfrak{M},[\widetilde{F}, \mathfrak{B}]=3\right\}$. Thus, $\mathscr{F}_{0} \neq \varnothing$, and $\Re_{1} \mathfrak{I}$ normalizes $\mathscr{F}_{0}$. Let

$\mathscr{F}=\left\{\widetilde{F}_{0} \mid \mathscr{F}_{0}\right.$ contains an element $F$ which inverts $\left.\Re_{2}\right\}$. Choose $\mathfrak{\mho}_{0} \in \mathscr{F}_{0}$. Then $\mathfrak{\mho}_{0} \nsubseteq \boldsymbol{C}(\mathfrak{B})$, and so $\mathfrak{\mho}_{0}$ does not centralize $\mathfrak{S}_{2} \Re_{2} / \mathfrak{F}_{2}$. Choose $F_{0} \in \mathfrak{F}_{0}-\mathfrak{\mho}_{0} \cap C(\mathfrak{B})$. Then $F_{0}$ inverts an element $R$ of $\mathfrak{S}_{\mathfrak{R}}$ of order 3. Hence, $\langle R\rangle=\mathfrak{R}_{2}^{H}$ for some $H$ in $\mathfrak{K}$ and so $\mathfrak{F}_{0}^{H^{-1}}=\tilde{\mathscr{F}} \in \mathscr{F}$. Thus, $\mathscr{F} \neq \varnothing$.

Choose $\tilde{\mho} \in \mathscr{F}$. Let $\tilde{\mho}_{1}=\tilde{\mho} \cap C(\mathfrak{B})$, and let $\widetilde{F} \in \mathfrak{F}$ invert $\Re_{2}$.

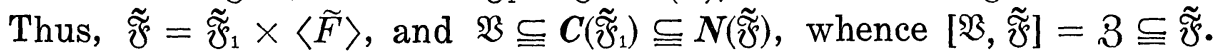
Let $\tilde{\mathscr{F}}=\mathscr{F}^{G}$. If $3=3^{\tilde{G}}$, then $\mathbb{F}=\widetilde{F}^{\widetilde{G}}=\tilde{\mathscr{F}}$, against Lemma 15.23. So $3^{\widetilde{G}}=\left\langle\widetilde{Z}_{1}\right\rangle \neq 3$, and $\left\langle Z, Z_{1}\right\rangle$ is a four-group in $\tilde{\mho}$.

Since $N(\tilde{\widetilde{\mho}})$ is transitive on $\tilde{\mho}-\left\langle Z_{1}\right\rangle$, it follows that $N(\tilde{\widetilde{\mho}}) \cap C(Z)$ is of index 6 in $N(\widetilde{\mathscr{\mho}})$. Let $\widetilde{\mathscr{G}}_{2}$ be a $S_{2}$-subgroup of $N(\tilde{\widetilde{\mho}}) \cap C(Z)$, and let $\mathbb{S}_{2}^{*}$ be a $S_{2}$-subgroup of $C(Z)$ which contains $\widetilde{\mathbb{S}}_{2}$. Since $C(Z)=\mathbb{S}_{2} \Re_{1}$, we have $\mathbb{S}_{2}^{*}=\mathscr{S}_{2}^{R}$ for some $R$ in $\Re_{1}$. Set $\widetilde{F}=\widetilde{\mathscr{F}}^{R^{-1}}$. Then $\widetilde{\mathscr{S}}_{2}^{R^{-1}}$ is a 
$S_{2}$-subgroup of $\boldsymbol{C}(\mathfrak{Z}) \cap N(\mathfrak{F})$ and $\widetilde{\mathscr{S}}_{2}^{R^{-1}} \subseteq \mathfrak{S}_{2}$. Also, $\mathfrak{F} \in \mathscr{F}$. Thus, $\mathfrak{F}$ has the following properties:

(a) $[\mathfrak{F}, \mathfrak{B}]=3, \mathfrak{F}=\mathfrak{F}^{G} \in \operatorname{ccl}_{\mathfrak{S}}(\mathfrak{F})$.

(b) $N(\mathfrak{F}) \cap \mathbb{S}_{2}$ is of index 2 in $\mathbb{F}_{2}$.

(c) $\left\langle Z_{1}\right\rangle=3^{G} \neq 3$.

(d) $\widetilde{F}=\boldsymbol{C}_{\mathfrak{F}}(\mathfrak{B}) \times\langle F\rangle$, where $F$ inverts $R_{2}$.

Set $\mathfrak{I}_{0}=\mathfrak{I} \cap N(\mathfrak{F})$. Since $\mathfrak{I} \subseteq \mathbb{S}_{2}$ and $\mathbb{S}_{2} \cap N(\mathfrak{F})$ is of index 2 in $\mathbb{S}_{2}$, it follows that $\mathfrak{I}_{0}$ is of index at most 2 in $\mathfrak{T}$. If $\mathfrak{I}_{0}=\mathfrak{I}$, then $\mathfrak{3}^{a} \subseteq Z(\mathfrak{I})$, as $Z(\mathfrak{T})$ is cyclic. This gives $\mathfrak{Z}^{G}=3$, against (c). Hence, $\left|\mathfrak{T}: \mathfrak{I}_{0}\right|=2$.

Set $\mathscr{S}_{0}=\mathfrak{S E}_{\mathfrak{S}} \cap \mathfrak{I}_{0}, \quad \mathscr{S}_{1}=\left[\mathfrak{S}_{\mathcal{E}}, \Re_{2}\right], \quad \mathscr{S}_{2}=C_{\mathfrak{f}}\left(\Re_{2}\right)$. Let $\left|\mathfrak{S}_{1}: \mathfrak{S}_{1}^{\prime}\right|=2^{2 f}$. Then $f$ is an integer and $\left|\boldsymbol{C}_{\mathfrak{f}_{1} / \mathfrak{f}_{1}^{\prime}}\left(F^{\prime}\right)\right|=2^{f}$. Since $\mathfrak{I}_{0}$ normalizes $\mathfrak{f}$ and centralizes $\mathfrak{U}=\left\langle Z, Z_{1}\right\rangle$, it follows that $C_{\widetilde{x}_{0}}(F)$ is of index at most 4 in $\mathfrak{I}_{0}$. Hence, $\left|\mathfrak{T}: C_{\mathfrak{x}}(F)\right| \leqq 8$. In particular, $f \leqq 3$.

Case 1. $f=3$.

We argue that $\mathfrak{B} \nsubseteq \mathfrak{S}_{1}^{\prime}$. Suppose false. Let $\mathfrak{Y}$ be a subset of $\mathfrak{S}_{1}$ of cardinal 8 such that the image of $\mathfrak{Y}$ in $\mathfrak{S}_{1} / \mathfrak{E}_{1}^{\prime}$ is a transversal to $\boldsymbol{C}_{\mathfrak{F}_{1} / \mathfrak{F}_{1}^{\prime}}(F)$ in $\mathfrak{S}_{1} / \mathfrak{S}_{1}^{\prime}$, and let $\mathfrak{B}_{0}$ be a subgroup of $\mathfrak{B}$ of order 2 distinct from 3. Then $\left|\mathfrak{Y \mathfrak { B } _ { 0 }}\right|=16$, and distinct elements of $\mathfrak{Y \mathfrak { B }}_{0}$ lie in distinct cosets of $C_{\mathfrak{f}}(F)$. This violates $\left|\mathfrak{S}_{\mathfrak{c}}: C_{\mathfrak{F}}(F)\right| \leqq 8$. So $\mathfrak{S}_{1}^{\prime} \cap \mathfrak{Y}=1$. Since $\mathfrak{B}$ is the only minimal normal subgroup of $\mathfrak{M}$ and since $\mathfrak{S}_{1}^{\prime} \triangleleft \mathfrak{M}$, we have $\mathfrak{S}_{1}^{\prime}=1$.

Let $\mathfrak{S}_{10}=\mathscr{S}_{1} \cap \mathfrak{F}_{0}$, so that $\mathfrak{S}_{10}$ is of index 2 (or 1) in $\mathfrak{S}_{1}$. Since $\left|\mathfrak{S}_{1}\right|=2^{6}$, we have $\left|\left[\mathfrak{S}_{10}, F\right]\right| \geqq 2^{2}$. But $\left[\mathfrak{S}_{10}, F\right] \leqq \mathfrak{S}_{1} \cap \mathfrak{F} \leqq C_{\Im}(\mathfrak{B})=$ $\left\langle Z, Z_{1}\right\rangle$, and so $\left[\mathscr{S}_{10}, F\right]=\left\langle Z, Z_{1}\right\rangle$. Thus, $\mathfrak{S}_{1} \subseteq C\left(Z_{1}\right)=N(\mathfrak{F})$, and so $\left[\mathfrak{S}_{1}, \mathfrak{F}\right] \subseteq \mathfrak{F}$. Since $|\mathfrak{F}|=8$, we have $\mathfrak{F}=\left[\mathfrak{H}_{1}, F\right]$, which is false, since $\mathfrak{F} \nsubseteq \mathfrak{K}$.

Case 2. $f=2$ and $\mathfrak{S}_{1} / \mathscr{S}_{1}^{\prime}$ is the direct product of 2 cyclic groups of order 4 .

Since $Z_{1}$ centralizes a subgroup of index 2 in $\mathfrak{I}$, it follows that $Z_{1} \in \mathfrak{S}_{\mathrm{C}}$. Let $Z_{1}=H_{1} H_{2}$, with $H_{i} \in \mathfrak{S}_{i}$. Then since $Z_{1}$ centralizes $\mathfrak{F}_{10}=$ $\mathfrak{H}_{1} \cap \mathfrak{S}_{0}$, lso does $H_{2}$. Since $\boldsymbol{C}_{\mathfrak{5}}\left(H_{2}\right)$ admits $\Re_{2}$, and since $\boldsymbol{C}_{\mathfrak{\xi}}\left(H_{2}\right)$ is of index at most 2 in $\mathscr{S}_{1}$, it follows that $H_{2} \in C\left(\mathscr{S}_{1}\right)$. Hence, $Z_{1} \in \boldsymbol{C}\left(\mathfrak{S}_{1}\right)$, and so $\mathfrak{S}_{1} \subseteq C\left(Z_{1}\right)=N(\mathfrak{F})$. Hence, $\left[\mathfrak{S}_{1}, F\right] \subseteq \widetilde{F}$. This is false, since $\widetilde{F}$ is elementary, and $\left[\mathfrak{S}_{1}, F\right]$ is cyclic of order 4 . So $\mathfrak{S}_{1}^{\prime} \neq 1$. Since $\mathfrak{B}$ is the only minimal normal subgroup of $\mathfrak{M}$, we have $\mathfrak{N} \subseteq \mathfrak{F}_{\mathfrak{1}}^{\prime}$.

Let $\mathscr{S}_{3}=\left[\mathfrak{S}_{1}^{\prime}, \mathfrak{F}_{1}\right]$. Then $\mathfrak{S}_{1}^{\prime} / \mathscr{S}_{3}$ is cyclic, so is centralized by $\Re_{2}$. Choose $H \in \mathfrak{S}_{1}^{\prime}$ such that $\mathfrak{S C}_{3} H$ is a generator for $\mathfrak{S C}_{1}^{\prime} / \mathfrak{S C}_{3}$, and set $\mathfrak{S}_{4}=$ $\left[\mathfrak{S}_{3}, \mathscr{S}_{1}\right]$. Then $\mathfrak{S}_{3}=\left[\mathscr{S}_{1}, H\right] \mathscr{S}_{4}$, and $\mathfrak{R}_{2}$ has no fixed points on $\mathfrak{S}_{3} / \mathscr{S}_{\mathcal{L}_{4}}$. Let $\mathscr{S}_{3} \subseteq \widetilde{\mathcal{F}}_{3} \subset \mathscr{S}_{4}$, with $\mathscr{S}_{3} / \tilde{\mathcal{F}}_{3}$ a chief factor of $\mathfrak{T R}_{2}$. If $\mathfrak{B} \subseteq \widetilde{\mathcal{F}}_{3}$, we get $\left|\mathscr{S}_{1}: C_{\mathfrak{F}_{1}}(F)\right| \geqq 16$. Hence, $\mathfrak{B} \nsubseteq \tilde{\mathfrak{V}}_{3}$, and since $\mathfrak{B}$ is the only minimal 
normal subgroup of $\mathfrak{I R}_{2}$, we get $\tilde{\mathfrak{F}}_{3}=1$. So $\operatorname{cl}\left(\mathscr{F}_{1}\right)=3$, and $\mathfrak{S}_{\mathfrak{I}}^{\prime}$ is abelian of type $\left(2^{a}, 2,2\right)$ for some $a \geqq 1$. If $a>1$, then $\mathfrak{S}_{1}^{\prime}$ has a characteristic subgroup of order 2 , against the fact that $\mathfrak{B}$ is the only minimal normal subgroup of $\mathfrak{M}$. So $a=1$.

Since $\mathfrak{F}_{1}$ is generated by 2 elements, and since $\mathscr{\mathcal { F }}_{1} \triangleleft \mathfrak{M}$, $\mathfrak{R}$ does not act faithfully on $\mathfrak{S}_{1_{1}}$. Since $\boldsymbol{C}\left(\mathfrak{F}_{1}\right) \triangleleft \mathfrak{M}$, it follows that $\boldsymbol{C}\left(\mathfrak{S}_{1}\right)$ has order divisible by 3 but not by 9 , and so $\Re_{1} \subseteq C\left(\mathscr{S}_{1}\right)$. Let $\mathfrak{K}^{1}=$ $\left[\mathfrak{S}_{2}, \mathfrak{R}_{1}\right] \triangleleft \mathfrak{M}$. Thus, $\mathfrak{S}^{1} \neq 1$, and so $\mathfrak{B} \cong \mathfrak{S}^{1}$. Since $\left[\mathfrak{S}_{1}, \mathfrak{R}_{1}\right]=1$, it follows that $\mathfrak{S C}^{1} \cap \mathfrak{S E}_{1} \subseteq\left[\mathfrak{S E}^{1}, \mathfrak{S E}^{1}\right]$, and so $\Re_{2}$ centralizes $\mathfrak{S E}^{1} /\left[\mathfrak{S E}^{1}, \mathfrak{S E}^{1}\right]$. Thus $\left[\Re_{2}, \mathfrak{S}^{1}\right]=1$, against $\mathfrak{B} \subseteq \mathfrak{S}^{1}$.

Case 3. $f=2, \mathfrak{S}_{1} / \mathscr{S}_{1}^{\prime}$ is elementary and $\mathfrak{S}_{1}^{\prime} \neq 1$.

If $Z_{1}$ centralizes $\mathfrak{S}_{1}$, then $\left[\mathfrak{F}_{1}, F\right]=C_{\mathfrak{F}}(\mathfrak{B})=\left\langle Z, Z_{1}\right\rangle$. This is impossible, since $\left|\left[\mathfrak{S}_{1}, F\right] \mathfrak{S}_{1}^{\prime} / \mathscr{S}_{1}^{\prime}\right| \geqq 4$, and $Z \in \mathfrak{S}_{1}^{\prime}$. So $Z_{1} \notin C\left(\mathscr{S}_{1}\right)$, whence $\mathfrak{I}=\mathfrak{I}_{0} \mathfrak{S}_{1}$. Let $\mathfrak{W}=\left\langle Z_{1}\right\rangle^{T}$ so that $|\mathfrak{W}|=4$, and $\mathfrak{W}=\left\langle Z_{1}, Z_{1}^{H}\right\rangle$ for some $H$ in $\mathfrak{K}$. Thus, $Z=Z_{1} Z_{1}^{H}$. Let $\mathfrak{W}^{*}=\mathfrak{W}^{\mathfrak{R}_{2}}$, so that $\mathfrak{W}^{*} \triangleleft \mathfrak{I} \mathfrak{R}_{2}$, $\mathfrak{W}^{*} \supset \mathfrak{B}$, and $\left|\mathfrak{W}^{*} / \mathfrak{B}\right| \leqq 2^{3}$. Since $\left[\mathfrak{S}_{2}, Z_{1}\right] \subseteq \mathfrak{B}$, it follows that $\mathfrak{S}^{\prime}$ centralizes $\mathfrak{S}^{*}$. We argue that $\mathfrak{W}^{*}$ is abelian. Suppose false. Now $\mathfrak{W}^{*}=\left\langle\mathfrak{B}, Z_{1}, Z_{1}^{R}, Z_{1}^{R^{-1}}\right\rangle$, where $\langle R\rangle=\mathfrak{R}_{2}$. Since $Z_{1}$ centralizes a subgroup of $\mathfrak{W}^{*}$ of index 2 , and $\left[Z_{1}, Z_{1}^{R}\right] \neq 1$, we get $\left[Z_{1}, Z_{1}^{R} Z_{1}^{R^{-1}}\right]=1$, and so $\left[Z_{1}, Z_{1}^{R}\right]=\left[Z_{1}, Z_{1}^{R^{-1}}\right] \in \mathfrak{S}^{\sharp}$. Thus, $\left[Z_{1}, Z_{1}^{R^{-1}}\right]^{R}=\left[Z_{1}^{R}, Z_{1}\right]=\left[Z_{1}, Z_{1}^{R}\right]$, so that $\left[Z_{1}, Z_{1}^{R}\right] \in \mathfrak{B} \cap C(\Re)=1$. so $\mathfrak{W}^{*}$ is abelian.

If $\left|\mathfrak{S}^{*}\right|=8$, then $\mathfrak{W}^{*}=\mathfrak{W}_{0} \times \mathfrak{B}$, where $\mathfrak{W}_{0}=\langle W\rangle=\boldsymbol{C}_{\mathfrak{B N}}\left(\mathfrak{R}_{2}\right)$ is of order 2. This implies that $Z_{1}=W Z$, since $\Re_{2} \nsubseteq C\left(Z_{1}\right)$, and $\boldsymbol{C}_{\mathfrak{g B}^{*}}(F)=$ $\left\langle W_{0}, Z\right\rangle \supseteqq\left\langle Z_{1}\right\rangle$. If $W$ and $Z_{1}$ are fused in $\mathfrak{S}$, say $Z_{1}=W^{H}$, then $\Re_{2}^{H}$ centralizes $Z_{1}$, so that $\mathfrak{F}$ centralizes $\Re_{2}^{H} \mathfrak{F} / \mathscr{F}=\Re_{2} \mathfrak{E} / \mathscr{S}$, which is false. Hence, $W$ and $Z_{1}$ are not fused in $\mathfrak{S}$. This implies that $\mathfrak{F}$ centralizes $\mathfrak{B}$ and does not map onto the stability group of the chain $\mathfrak{W}^{*} \supset \mathfrak{V} \supset 1$. Hence, $\mathfrak{W}^{*} \subseteq Z(\mathfrak{S})$. Since $\mathfrak{I R}_{2}=\mathfrak{S}_{\mathfrak{C}} \cdot N_{\mathfrak{x \mathfrak { N }}_{2}}\left(\mathfrak{R}_{2}\right)$, it follows that $\mathfrak{W}_{0} \subseteq Z(\mathfrak{I})$, which is false. Hence, $\left|\mathfrak{W}^{*}\right| \geqq 2^{4}$.

Since $\left\langle Z_{1}, \mathfrak{B}\right\rangle \triangleleft \mathfrak{F}$, it follows that $\left|\left[\mathfrak{S}^{*}, \mathfrak{R}_{2}\right]\right|=2^{4}$, and so $\left|\left[\mathfrak{S}^{*}, \Re_{2}, F\right]\right|=2^{2}$. Since $\left[\mathfrak{W}^{*}, \mathfrak{R}_{2}, F\right] \leqq \widetilde{F} \cap C(V)=\left\langle Z, Z_{1}\right\rangle$, it follows that $\mathfrak{W}^{*}=\left[\mathfrak{W}^{*}, \mathfrak{R}_{2}\right]$ is of order $2^{4}$. Since $\left|\mathfrak{F}_{\mathfrak{S}}: \boldsymbol{C}_{\mathfrak{p}}\left(Z_{1}\right)\right|=2$, it follows that $\left|\mathfrak{S}_{\mathfrak{C}}: C_{\mathfrak{F}}\left(\mathfrak{W}^{*}\right)\right| \leqq 4$. Let $\tilde{\mathfrak{F}}=C_{\mathfrak{F}}\left(\mathfrak{W}^{*}\right)$. Then $[\tilde{\mathfrak{S}}, F] \leqq \widetilde{F} \cap \boldsymbol{C}(\mathfrak{B})=$ $\left\langle Z, Z_{1}\right\rangle$, and so $F$ centralizes $\tilde{\mathscr{F}} / \mathfrak{W}^{*}$, whence $\Re_{2}$ centralizes $\tilde{\mathfrak{F}} / \mathfrak{W}^{*}$, and so $\tilde{\mathfrak{F}}=C_{\mathfrak{F}}\left(\Re_{2}\right) \times \mathfrak{W}^{*}$. Since $Z \in \mathfrak{S}_{1}^{\prime}$, it follows that $\mathfrak{W}^{*} \subset \mathfrak{S}_{1}$, and so $\Re_{2}$ does not centralize $\mathscr{S} / \tilde{\mathcal{H}}$, whence $\mathscr{S} / \tilde{\mathcal{H}}$ is a four-group. Choose $H \in C_{\mathfrak{5}}\left(Z_{1}\right)-\tilde{\mathfrak{F}}$, and let $K=H^{R}, Z_{2}=Z_{1}^{R}$. Then $\left[H, Z_{1}\right]=1=\left[K, Z_{2}\right]=$ $\left[H K, Z_{1} Z_{2}\right]$, and so $\left|\left[\mathfrak{S}, \mathfrak{S}^{*}\right]\right| \leqq 2$. Since $\left[\mathfrak{\mathcal { E }}, \mathfrak{W}^{*}\right] \subseteq \mathfrak{B}$, and $\left[\mathfrak{S}, \mathfrak{W}^{*}\right]$ admits $\Re_{2}$, we get $\mathfrak{F}=\tilde{\mathscr{F}}$. This contradiction shows that this case does not arise.

Case 4. $f=2$ and $\mathfrak{S}_{1}$ is elementary. 
Write $Z_{1}=H_{1} H_{2}$, where $H_{i} \in \mathfrak{S}_{i}$. (Since $Z_{1}$ centralizes a subgroup of index 2 in $\mathfrak{S}$, it follows that $Z_{1} \in \mathfrak{F}$.) Since $Z_{1}$ centralizes a subgroup of $\mathscr{S}_{1}$ of index 2 , so does $H_{2}$. Since $C_{\mathfrak{\vartheta}_{1}}\left(H_{2}\right)$ admits $\Re_{2}$, it follows that $H_{2} \in \boldsymbol{C}\left(\mathfrak{F}_{1}\right)$, whence $Z_{1} \in \boldsymbol{C}\left(\mathfrak{F}_{1}\right)$, whence $\left[\mathfrak{S}_{1}, F\right] \leqq C(\mathfrak{B}) \cap \widetilde{F}=\left\langle Z, Z_{1}\right\rangle$, and so $\left[\mathfrak{S}_{1}, F\right]=\left\langle Z, Z_{1}\right\rangle$. Let $\mathfrak{S}_{3}=C_{\mathfrak{S}_{2}}\left(\mathfrak{F}_{1}\right)$. Then $\quad \mathfrak{S}_{3} \triangleleft \mathfrak{M}$, and

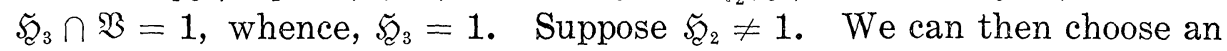
involution $H$ of $\mathfrak{S}_{2}$ which is centralized by $F$. Hence, $H \in C(\langle F, Z\rangle) \subseteq$ $N(\mathfrak{F})=C\left(Z_{1}\right)$, and so $C_{\mathfrak{F}_{1}}(H)$ contains $\mathfrak{B}$ properly, whence $H$ centralizes $\mathfrak{S}_{1}$. This violates $\mathscr{S}_{3}=1$. We conclude that $\mathscr{S}_{2}=1$, and so $\mathfrak{S}_{2}=\mathscr{S}_{1}$ is elementary of order $2^{4}$. But then $\mathfrak{S}$ is 2-reducible in $\mathfrak{M}$, against $|\mathfrak{B}|=4$. We have thus completed a proof of the following result.

\section{THEOREM 15.2. $\mathfrak{T}$ is a $S_{2}$-subgroup of 8 .}

The preceding theorem did not come easily, but we would not be able to save any effort by first considering the elements of $\mathscr{C} \mathscr{S}$ (S) which contain a $S_{2}$-subgroup of (s). The configuration we have just analysed is unavoidable within the framework of the present techniques.

We must once again turn to the model given in $\S 13$ to complete the analysis of the present situation. We have already done most of the hard work, but there are a few alterations necessary in the arguments of $\S 13$. Also, there is some difficulty in determining enough information about the isomorphism class of $\Re$. This difficulty arises because of the possibility that $\Re$ may be of maximal class. So we must bring to bear the detailed results of Blackburn about the 3groups of maximal class.

We retain the notation introduced at the beginning of this section. The argument of Lemma 15.6 still applies to give us $\Re \subseteq \mathfrak{M}^{\prime}$. Thus, if $\mathfrak{D}$ is a $S_{\{2,3\}}$, -subgroup of $\mathfrak{M}$ permutable with $\mathfrak{R}$, then $\mathfrak{D} \mathfrak{R}=\mathfrak{D} \times \mathfrak{R}$. Set $\Re=\mathfrak{D} \Re$. Of course, $D$ is a $Z$-group.

We need some additional notation. Let $\Re_{0}=\Re \cap \boldsymbol{O}_{3^{\prime}, 3}(\mathfrak{M})$, and choose $\Re_{1}$ char $\Re_{0}, \Re_{1}$ of exponent $3, \Re_{1}^{\prime} \subseteq \mathbb{Z}\left(\Re_{1}\right)$, such that $\operatorname{ker}\left(\operatorname{Aut}\left(\mathfrak{R}_{0}\right) \rightarrow \operatorname{Aut}\left(\mathfrak{R}_{1}\right)\right)$ is a 3-group. Let $\mathfrak{X}=\boldsymbol{O}_{3^{\prime}}(\mathfrak{M}) \Re_{1} / \boldsymbol{O}_{3^{\prime}}(\mathfrak{M}) \boldsymbol{D}\left(\Re_{1}\right)$. Since $\left|\Re_{1}\right|=3^{2}$ or $3^{3}$, it follows that $|\mathfrak{X}|=3^{2}$; and $\boldsymbol{C}_{\mathfrak{M}}(\mathfrak{X}) \subseteq \boldsymbol{O}_{3^{\prime}, 3}(\mathfrak{M})$.

\section{LEMMA 15.27. $\mathfrak{S}_{0} \Re_{0} \triangleleft \mathfrak{M}$.}

Proof. We note that since $\mathfrak{S}_{2} / \mathscr{S}_{0}$ acts faithfully on $\boldsymbol{O}_{2,2}(\mathfrak{M}) / \mathscr{F}_{0}$, and since $\mathfrak{S}_{\mathcal{C}} / \mathfrak{S}_{0}$ centralizes a $S_{3^{-}}$-subgroup of $\boldsymbol{O}_{2,2},(\mathfrak{M}) / \mathfrak{S}_{0}$, while a $S_{3^{\prime}}$, subgroup of $\boldsymbol{O}_{2,2}(\mathfrak{M}) / \mathscr{Y}_{0}$ is a $Z$-group, it follows that $\left[\mathfrak{H}_{2}, \Re\right] \subseteq \mathfrak{S}_{0}$. Hence, $\mathfrak{S}_{0} \Re_{0}=\boldsymbol{O}_{3}\left(\mathfrak{T} \mathfrak{R} \bmod \mathscr{S}_{2}\right)$. Since $\mathfrak{M}=\mathfrak{T} \mathfrak{R}$, and $[\mathfrak{R}, \mathbb{D}]=1$, the lemma follows. 
LEMMA 15.28. If $\boldsymbol{C}_{\mathfrak{R}}(\mathfrak{B})$ is cyclic, and $\mathfrak{I} / \mathfrak{S}$ contains a quaternion subgroup, then $J(\mathfrak{T})=\boldsymbol{J}(\mathfrak{S})$, and $N(J(\mathfrak{T})) \subseteq \mathfrak{M}$.

Proof. Since $\mathfrak{F}_{0} \mathfrak{R}_{0} \triangleleft \mathfrak{M}$, so also $\mathfrak{S}_{0} \mathfrak{R}_{1} \triangleleft \mathfrak{M}$. Let $\mathfrak{M}_{1}=\mathfrak{T} \mathfrak{R}_{1}$. We may view $\mathfrak{B}$ as a $F_{2} \mathfrak{M}_{1} / \mathfrak{S}_{0}$-module. Since $\mathfrak{S}_{0} \Re_{1} / \mathscr{S}_{0} \boldsymbol{D}\left(\mathfrak{R}_{1}\right)$ is an irreducible module for $\mathfrak{T} / \mathfrak{S}$, it follows that $\boldsymbol{C}_{\Re_{1}}(\mathfrak{B}) \subseteq \boldsymbol{D}\left(\Re_{1}\right)$. Since $\mathfrak{S}_{\mathfrak{2}} \mathfrak{R}_{1} / \mathfrak{S}_{0}=$ $\mathfrak{S} / \mathscr{S}_{0} \times \Re_{1} \mathfrak{S}_{0} / \mathscr{S}_{0}$, the $P \times Q$-lemma implies that if $\mathfrak{V}_{0}=C_{\mathfrak{g}}(\mathfrak{S})$, then $\boldsymbol{C}_{\mathfrak{R}_{1}}\left(\mathfrak{B}_{0}\right)=\boldsymbol{C}_{\mathfrak{R}_{1}}(\mathfrak{B}) \leqq D\left(\mathfrak{R}_{1}\right)$. Let $\mathfrak{A}$ be an abelian subgroup of $\mathfrak{I}$ of maximal order (or rank), and let $\mathfrak{B}=\mathfrak{U} \cap \mathfrak{S}$. Then $\mathfrak{U} / \mathfrak{B}$ is isomorphic to a subgroup of $G L(2,3)$. Let $\mathfrak{B}_{1}=\mathfrak{A} \cap \mathfrak{B}_{0}=\mathfrak{B} \cap \mathfrak{B}_{0}$, so that $\left|\mathfrak{B}_{0} \mathfrak{B}\right|=$ $|\mathfrak{B}| \cdot\left|\mathfrak{B}_{0}: \mathfrak{B}_{1}\right|$, and since $\mathfrak{B}_{0} \mathfrak{B}$ is abelian, we get

$$
\left|\mathfrak{B}_{0}: \mathfrak{B}_{1}\right| \leqq|\mathfrak{N}: \mathfrak{B}| \text {. }
$$

If $|\mathfrak{X}: \mathfrak{B}|=8$, then $\mathfrak{U} / \mathfrak{B}$ is cyclic. Since $\mathfrak{X} / \mathfrak{B}$ acts faithfully on $\mathfrak{B}_{0}$, we have $\left|\mathfrak{B}_{0}: \mathfrak{B}_{1}\right| \geqq 2^{4}$. If $\mathfrak{X} / \mathfrak{B}$ is cyclic of order 4 , then the only possibility is that $\mathfrak{B}_{0}=\mathfrak{B}_{00} \times \mathfrak{B}_{01}$, where $\mathfrak{B}_{00} \subseteq \mathfrak{U}$, and $\mathfrak{B}_{01}$ is an indecomposable $F_{2} \mathfrak{A}$-module of order 8 . Thus, if $\mathfrak{A}=\langle\mathfrak{B}, T\rangle$, then $\boldsymbol{C}_{\mathfrak{B}_{0}}\left(T^{2}\right)$ is a hyperplane of $\mathfrak{B}_{0}$. This is false, since $T^{2}$ inverts $\mathfrak{X}$. If $\mathfrak{X} / \mathfrak{B}$ is a four-group, there are two cases.

Case 1. $\left|\mathfrak{R}_{1}\right|=3^{3}$ and $\mathfrak{R}_{1}$ acts faithfully on $\mathfrak{B}_{0}$.

Since $\mathfrak{X} / \mathfrak{B}$ is a four-group, and $\mathfrak{N} / \mathfrak{B} \cong \mathfrak{N} \mathfrak{F} / \mathfrak{S}$, it follows that $\mathfrak{X}$ has an element $T$ which inverts $\Re_{1}^{\prime} \mathfrak{Y}_{0} / \mathfrak{S}_{0}$. Since $\left|\left[\mathfrak{V}_{0}, \Re_{1}^{\prime}\right]\right| \geqq 2^{6}$, we have $\left|\mathfrak{B}_{0}: C_{\mathfrak{B}_{0}}(T)\right| \geqq 2^{3}$, and so $\left|\mathfrak{B}_{0}: \mathfrak{B}_{1}\right| \geqq\left|\mathfrak{B}_{0}: C_{\mathfrak{B}_{0}}(T)\right|>|\mathfrak{R}: \mathfrak{B}|$.

Case 2. $\mathfrak{R}_{1} / \boldsymbol{C}_{\mathfrak{\Re}_{1}}\left(\mathfrak{B}_{0}\right)$ is of order $3^{2}$.

Since $\boldsymbol{D}\left(\mathfrak{R}_{1}\right) \subseteq \boldsymbol{C}\left(\mathfrak{B}_{0}\right)$, we may view $\mathfrak{B}_{0}$ as a $F_{2} X$-module. Since $\mathfrak{I} / \mathfrak{S}$ contains a quaternion group, $\mathfrak{T}$ permutes transitively the 1-dimensional subspaces of $\mathfrak{X}$. Hence, $\left|\left[\mathfrak{B}_{0}, \mathfrak{X}\right]\right| \geqq 2^{8}$. Since $\mathfrak{X} / \mathfrak{B}$ is a fourgroup, there is $T$ in $\mathfrak{X}$ which inverts $\mathfrak{X}$. So $\left|\mathfrak{B}_{0}: \mathfrak{B}_{1}\right| \geqq\left|\mathfrak{B}_{0}: \boldsymbol{C}_{\mathfrak{B}_{0}}(T)\right| \geqq$ $2^{4}>|\mathfrak{Q}:: \mathfrak{B}|$.

If $|\mathfrak{U} / \mathfrak{B}|=2$, then $\mathfrak{A}=\langle\mathfrak{B}, T\rangle$, and $\boldsymbol{C}_{\mathfrak{B}_{0}}(T)$ is forced to be a hyperplane of $\mathfrak{B}_{0}$. This is false, since $\mid\left[\mathfrak{B}_{0}, \Re\right] \geqq 2^{4}$ for every subgroup $\widetilde{\mathfrak{R}}$ of $\Re_{1}$ which does not centralize $\mathfrak{V}_{0}$, and since $T$ inverts $\mathfrak{S}_{0} \widetilde{\mathfrak{R}} / \mathscr{Y}_{0}$ for soma such $\widetilde{\Re}$. So $J(\mathfrak{I})=J(\mathfrak{E})$. Since $\mathfrak{S} \Re \subseteq N(J(\mathfrak{I}))$, the proof is complete, by Lemma 14.20 .

LEMMA 15.29. Suppose $\mathfrak{B}$ is a non cyclic 3-subgroup of $\mathfrak{M}$, $\mathfrak{S}_{1} \in \mathcal{U}_{\mathfrak{N}}(\mathfrak{B} ; 2)$, and $\mathfrak{F}_{1}$ contains an elementary subgroup of order 8 . Then $\mathfrak{S}_{1} \mathfrak{B} \in \mathscr{M}^{*}$.

Proof. We assume without loss of generality that $|\mathfrak{B}|=3^{2}$. Let 
$\mathscr{S}=\left\{\subseteq \mid \mathscr{S}_{1} \mathfrak{B} \leqq \subseteq \subseteq \mathscr{B}, \mathscr{S}\right.$ is solvable, $\left.\mathfrak{S} \nsubseteq \mathfrak{M}\right\}$. Suppose $\mathscr{S} \neq \varnothing$. Choose $\mathscr{S}$ in $\mathscr{S}$ with $|\mathfrak{S} \cap \mathfrak{M}|_{2}$ maximal, and with this restriction, minimize $\mathfrak{S}_{\text {. Let }} \mathfrak{S}_{1}=\boldsymbol{O}_{3^{\prime}}(\mathfrak{S})$, and let $\mathfrak{S}_{2}$ be a $S_{2}$-subgroup of $\mathfrak{S}_{1}$ which admits $\mathfrak{B}$ and contains a $S_{2}$-subgroup $\tilde{\mathfrak{S}}_{2}$ of $\boldsymbol{O}_{3^{\prime}}(\mathfrak{S}) \cap \mathfrak{M}$. We argue that $N(\mathfrak{Y}) \subseteq \mathfrak{M}$ for every non identity characteristic subgroup $\mathfrak{Y}$ of $\tilde{\mathfrak{S}}_{2}$. This is clear by Lemma 14.20 if $\widetilde{\mathfrak{S}}_{2} \in N_{\mathfrak{M}}^{*}(\mathfrak{B} ; 2)$, so suppose $\widetilde{\mathfrak{S}}_{2} \notin N_{\mathfrak{N}}^{*}(\mathfrak{B} ; 2)$. Since $\mathfrak{H}_{1} \subseteq O_{3^{\prime}}(\mathfrak{S})$ (as $3 \in \pi_{2}$ ), it follows that $\widetilde{\mathfrak{S}}_{2} \neq 1$, and so $\left|N_{\mathfrak{n}}(\mathfrak{Y})\right|_{2}>|\subseteq \cap \cap M|_{2}$, whence $N(\mathfrak{Y}) \subseteq \mathfrak{M}$ by maximality of $|\subseteq \cap \mathfrak{M}|_{2}$. Since $N\left(\widetilde{S}_{2}\right) \subseteq \mathfrak{M}$, we get $\widetilde{\subseteq}_{2}=\widetilde{S}_{2}$. Since $\mathbb{L}=\boldsymbol{O}_{3^{\prime}}(\mathfrak{S})$ is a $3^{\prime}$-group, minimality of $\mathfrak{S}$ gives $\mathfrak{Z}=\mathfrak{S}_{2} \mathfrak{N}$, where $\mathfrak{Z}$ is a $q$-group for some prime $q \geqq 5$, and $\mathfrak{Q}$ admits $\mathfrak{B}$. Since $\mathfrak{S}_{1}$ contains an elementary subgroup of order 8 , we get that $\mathfrak{Q}$ is cyclic. Hence, $\boldsymbol{O}_{2}(\mathfrak{S}) \neq 1$. By Lemma $14.13,[\mathfrak{B}, \mathfrak{Q}]=1$. Since $C(\mathfrak{B})=\mathfrak{M}$, we get $\mathfrak{D} \subseteq \mathfrak{M}$, and so $\subseteq \subseteq \mathfrak{M}$. The proof is complete.

Let $\hat{\mathfrak{M}}=\left\{\mathrm{M} \mid M \in \mathfrak{S}_{0} \Re_{0}, M\right.$ has order $3, M$ is real and $N(\langle M\rangle) \subseteq$ $\mathfrak{M}\}$.

Lemma 15.30. Suppose $M \in \hat{\mathfrak{M}}, \mathfrak{B}=\langle M\rangle$, and $\boldsymbol{C}_{\mathfrak{r}}(\mathfrak{B})$ is cyclic. If $\mathfrak{Q} \in \mathrm{K}_{\mathfrak{N}}^{*}(\mathfrak{B} ; 2)$, then the following hold:

(a) $M$ is real in $\boldsymbol{N}_{\mathfrak{m}}($ \&).

( b ) $N(\mathbb{Q}) \subseteq \mathfrak{M}$.

(c) $\mathfrak{R B} \in \mathscr{M}^{*}$.

Proof. Let $\mathbb{2}^{*}=\boldsymbol{O}_{3^{\prime}}(\mathfrak{M}) \mathbb{R} \mathfrak{B}$. Since $\mathbb{2}^{*}$ has a normal 3-complement, $N_{\mathbb{R}^{*}}^{*}(\mathfrak{B} ; 2)=\left\{\mathbb{Q}^{C} \mid C \in C(\mathfrak{B}) \cap \boldsymbol{O}_{3^{\prime}}(\mathfrak{M})\right\}$, and so \& contains a $S_{2}$-subgroup of $\mathfrak{R}^{*}$. We assume without loss of generality that $\mathbb{\complement} \subseteq \mathfrak{I}, \mathfrak{B} \subseteq \Re$. Thus,

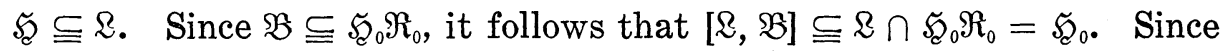
$\mathfrak{L} \in N_{\mathfrak{N}}^{*}(\mathfrak{B} ; 2)$, it follows that $\mathbb{R} / \mathscr{F}_{0}$ is a $S_{2}$-subgroup of $C_{\mathfrak{M} / \mathscr{F}_{0}}(\mathfrak{B})$. Since $M$ is real in $\mathfrak{M}$, there is $X$ in $\mathfrak{M}$ with $M^{X}=M^{-1}$. Let $\boldsymbol{C}_{\mathfrak{M} / \mathfrak{F}_{0}}(\mathfrak{B})=$ $\mathfrak{M}_{0} / \mathfrak{L}_{0}$, and let $\mathfrak{M}_{1}=\left\langle\mathfrak{M}_{0}, X\right\rangle$. Let $\mathfrak{Q}_{1}$ be a $S_{2}$-subgroup of $\mathfrak{M}_{1}$ which contains $\mathbb{R}$. Thus, $\left|\mathbb{R}_{1}: \mathbb{R}\right|=2$, and $\mathfrak{F}_{0} \mathfrak{B} \backslash \mathfrak{R}_{1} \mathfrak{B}$. Thus, (2) holds.

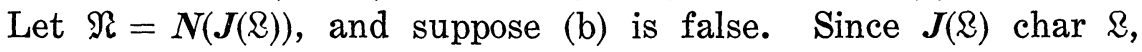
we have $\mathfrak{N} \nsubseteq \mathfrak{M}$. Let $\mathfrak{R}_{0}$ be a $S_{2,3}$-subgroup of $\mathfrak{R} \cap \mathfrak{M}$ which contains RSB, and let $\mathfrak{R}_{1}$ be a $S_{2,3}$-subgroup of $\mathfrak{N}$ which contains $\mathfrak{N}_{0}$. Let $\mathfrak{L} \subseteq \mathfrak{L}_{0} \subseteq \mathfrak{Z}_{1}, \mathfrak{B} \subseteq \mathfrak{B}_{0} \subseteq \mathfrak{B}_{1}$, where $\mathfrak{R}_{i}$ is a $S_{2}$-subgroup of $\mathfrak{R}_{i}$, and $\mathfrak{B}_{i}$ is a $S_{3}$-subgroup of $\mathfrak{R}_{i}$. If $\mathfrak{B}_{1}$ is non cyclic, then $\mathfrak{B}$ is contained in an elementary subgroup $\mathfrak{B}^{*}$ of $\mathfrak{B}_{1}$ of order 9 . Since $M \in \hat{\mathfrak{M}}$, we have $\mathfrak{B}^{*} \subseteq \mathfrak{M}$. Since $\mathfrak{F}_{0} \subseteq \mathfrak{Q} \subseteq \mathfrak{R}$, Lemma 14.20 gives $\mathfrak{N} \subseteq \mathfrak{M}$. Thus, $\mathfrak{B}_{1}$ is cyclic, and $\mathfrak{B}_{1} \subseteq C(\mathfrak{B}) \subseteq \mathfrak{M}$.

Let $\mathbb{R}_{1}=\boldsymbol{O}_{2}\left(\mathfrak{N}_{1}\right)$. Thus, $\mathbb{L}^{1} \cap \mathfrak{M}=\mathbb{R}$, since $\mathfrak{L} \in N_{\mathfrak{N}}^{*}(\mathfrak{B} ; 2)$. Suppose $\mathfrak{R}_{1}=\mathfrak{R}$. Since $\mathfrak{R}^{1}$ is of index 2 in $\mathfrak{R}_{1}$, we get from (a) that $\mathfrak{R}_{1} \subseteq \mathfrak{M}$. Since $\mathfrak{B} \subseteq \mathfrak{N}^{\prime}$ and a $S_{\{2,3\}}$-subgroup of $\mathfrak{R}$ is a $Z$-group, we have $\mathfrak{R}=\mathfrak{N}_{1} \cdot \boldsymbol{C}_{\mathfrak{r}}(\mathfrak{B}) \subseteq \mathfrak{M}$.

Suppose $\mathfrak{L}^{1} \supset \mathbb{R}$. Since $C(\mathfrak{B}) \subseteq \mathfrak{M}$, we get that $\left|\mathbb{R}^{1}: \mathbb{R}\right|$ is a square, 
and $\left|\mathbb{R}^{1}: \mathbb{R}\right| \geqq 4$. Hence, $\left|\mathbb{R}_{1}: \mathbb{R}\right| \geqq 8$. By Lemma 14.20 , we have $\mathbb{R} \supset \mathfrak{S}$, and so $\left|\mathbb{R}_{1}: \mathfrak{S}\right| \geqq 16$. Since $\mathfrak{T}$ is a $S_{2}$-subgroup of $\mathbb{S},|\mathfrak{I}: \mathfrak{S}| \geqq 16$. Thus, $\mathfrak{I} / \mathfrak{S}$ contains a quaternion group. Since $\mathfrak{S} \subseteq \mathbb{R} \subseteq \mathfrak{I}$, we get $\boldsymbol{J}(\mathfrak{T})=\boldsymbol{J}(\mathfrak{R})=\boldsymbol{J}(\mathfrak{S})$, and so $(\mathrm{b})$ follows from Lemma 15.28.

Now suppose $\mathbb{R} \subseteq \subseteq$ , and $\subseteq$ is a solvable subgroup of 85 . If $S_{3}$-subgroups of $\subseteq$ are non cyclic, then since $C(\mathfrak{B}) \subseteq \mathfrak{M}, S_{3}$-subgroups of $\subseteq \cap \mathfrak{M}$ are non cyclic, and so we get $\subseteq \subseteq \mathfrak{M}$, by Lemma 14.20. Suppose $\mathfrak{S}_{3}$ is a cyclic $S_{3}$-subgroup of $\subseteq$ which contains $\mathfrak{B}$. Then $\mathfrak{S}_{3} \subseteq C(\mathfrak{B}) \subseteq \mathfrak{M}$. Thus, $\mathbb{Z}$ is a $S_{2}$-subgroup of $\mathfrak{S}_{0}=\boldsymbol{O}_{3^{\prime}}(\mathfrak{S})$. Since $N_{\mathcal{S}}(\mathbb{R})$ contains a $S_{2,3}$-subgroup of $\subseteq$, we get that $|\subseteq: \subseteq \cap \mathfrak{M}|$ is prime to 6 .

Suppose $\subseteq \nsubseteq \mathfrak{M}$. In this case, there is a prime $p \geqq 5$ such that a $S_{p}$-subgroup $\mathfrak{P}$ of $\mathfrak{S}$ is permutable with $\mathfrak{B}$ and with $\mathfrak{L}$ and is not contained in $\mathfrak{M}$. Since $\sigma_{2}=\{3\}$ and $\boldsymbol{O}_{2}(\subseteq) \neq 1$, $\mathfrak{P}$ is cyclic. Since $\boldsymbol{C}(\mathfrak{B}) \subseteq \mathfrak{M}$ and $\mathfrak{P} \nsubseteq \mathfrak{M}$, $\mathfrak{B} \mathfrak{P}$ is a Frobenius group. Hence, $\mathfrak{B} \nsubseteq \widetilde{S}^{\prime}$, and so $\mathbb{R}$ is a $S_{2}$-subgroup of $\mathfrak{S}$. Let $\mathfrak{S}^{*}=\mathbb{R}$. Since $p \geqq 5$, there is $\mathfrak{Y} \in\{Z(\mathbb{R}), J(\mathfrak{Q})\}$ with $\mathfrak{Y} \triangleleft \mathfrak{S}^{*}$. Let $\widetilde{\subseteq}=N(\mathfrak{Y})$. Then $\widetilde{\subseteq} \nsubseteq \mathfrak{M}$, $\widetilde{\subseteq}$ is solvable, and $\mathfrak{B} \subseteq \widetilde{\Phi}^{\prime}$. The preceding argument applied with $\widetilde{\varsigma}$ in the role of $\subseteq$ completes the proof.

LEMmA 15.31. Suppose $M \in \hat{\mathfrak{M}}, \boldsymbol{C}_{\mathfrak{r}}(\mathfrak{B})$ is cyclic, and $\mathfrak{H}_{1}$ is a subgroup of $\mathfrak{S}_{0}$ normalized by $M$. If $\mathfrak{S}_{1}$ contains an elementary subgroup of order 8, then $\mathfrak{S}_{1}\langle M\rangle \in \mathscr{M}^{*}$.

Proof. Let $\mathscr{S}=\left\{\mathfrak{S} \mid \mathfrak{S}_{1} \mathfrak{M} \subseteq \subseteq \subseteq \mathbb{S}, \quad \mathfrak{S}\right.$ is solvable, $\left.\mathfrak{S} \nsubseteq \mathbb{M}.\right\}$. Suppose $\mathscr{S} \neq \varnothing$. Choose $\mathscr{S} \in \mathscr{S}$ such that $|\mathfrak{S} \cap \mathfrak{M}|_{2}$ is maximal, and $\subseteq$ is minimal with this restriction. By Lemma 15.29, $S_{3}$-subgroups of $\subseteq$ are cyclic. Since $M \in \hat{\mathfrak{M}}, \boldsymbol{C}(M) \subseteq \mathfrak{M}$, and so $|\subseteq: \subseteq \cap \mathfrak{M}|$ is prime to 3. Let $\mathfrak{S}_{0}=\boldsymbol{O}_{3^{\prime}}(\mathfrak{S})$, and let $\mathfrak{S}_{2}$ be a $S_{2}$-subgroup of $\mathfrak{S}_{0}$ which contains a $S_{2}$-subgroup $\widetilde{S}_{2}$ of $\widetilde{S}_{0} \cap \mathfrak{M}$. Since $\mathfrak{S}_{1} \subseteq \widetilde{S}_{0} \cap \mathfrak{M}$, we have $\widetilde{\mathfrak{S}}_{2} \neq 1$. If $\widetilde{\mathfrak{S}}_{2} \in N_{\mathfrak{N}}^{*}(M ; 2)$, then by Lemma 15.30 , we have $\subseteq \leqq \mathfrak{M}$. So $\left.\widetilde{\subseteq}_{2} \nsubseteq N_{\mathfrak{M}}^{*}(\langle M\rangle) ; 2\right)$. Maximality of $|\subseteq \cap \mathfrak{M}|_{2}$ then gives $N(\mathfrak{Y}) \subseteq \mathfrak{M}$ for every non identity characteristic subgroup $\mathfrak{Y}$ of $\widetilde{\Im}_{2}$. Hence, $\widetilde{\Im}_{2}=\widetilde{\Im}_{2}$. Minimality of $\mathfrak{S}$ gives $\mathfrak{S}_{0}=\mathfrak{S}_{2} \mathfrak{Q}$, where $\mathfrak{Q}$ is a $q$-group for some prime $q \geqq 5$. Since $\mathfrak{K}_{1}$ contains an elementary subgroup of order 8 , we get that $\mathfrak{D}$ is cyclic. Hence, $\boldsymbol{O}_{2}\left(\mathfrak{S}_{0}\right) \neq 1$. By maximality of $|\subseteq \cap \mathfrak{M}|_{2}$, it follows that $\boldsymbol{O}_{2}\left(\mathfrak{S}_{0}\right) \supseteqq \mathscr{S}_{0}$. Hence, $\boldsymbol{O}_{2^{\prime}}\left(\mathfrak{S}_{0}\right)=1$. But then, $\mathfrak{S}_{0}=$ $\left(\Im_{0} \cap \boldsymbol{C}\left(\boldsymbol{Z}\left(\mathfrak{S}_{2}\right)\right)\right)\left(\Im_{0} \cap \boldsymbol{N}\left(\boldsymbol{J}\left(\mathfrak{S}_{2}\right)\right)\right) \subseteq \mathfrak{M}$, so $\subseteq \subseteq \mathfrak{M}$. The proof is complete.

\section{LEMMA 15.32 .}

(a) If $\Re_{0}$ contains an elementary subgroup $\mathfrak{B}$ of order 9 such that $N_{\mathfrak{m}}(\mathfrak{B})$ contains an element which inverts $\mathfrak{B}$, then $\hat{\mathfrak{M}} \neq \varnothing$.

(b) If $|\mathfrak{I} / \mathfrak{S}| \neq 2$, then $\hat{\mathfrak{M}} \neq \varnothing$. 
Proof. (a ) By Theorems 13.5, 13.6, 13.7, M contains a non cyclic normal elementary abelian 2-subgroup $\mathfrak{F}$. So $\mathfrak{B}$ normalizes some fourgroup $\mathfrak{F}$ of $\mathfrak{M}$. Let $\mathfrak{B}_{0}=C_{\mathfrak{B}}(\mathfrak{F})$. If $\mathfrak{B}=\mathfrak{B}_{0}$, then every element of $\mathfrak{B}^{*}$ is in $\hat{\mathfrak{M}}$ by Lemma 14.21 If $\left|\mathfrak{B}_{0}\right|=3$, then $\mathfrak{B}_{0}=\langle B\rangle$ and $B \in \hat{\mathfrak{M}}$, by Lemma 14.22. So (a) holds.

As for (b), since $\Re \subseteq \mathfrak{M}^{\prime}$, we have $|\mathfrak{I} / \mathfrak{S}| \geqq 2$. Suppose $|\mathfrak{I} / \mathfrak{S}| \geqq 4$. As $\mathfrak{Y} / \mathfrak{K}$ acts faithfully on $\mathfrak{S}_{\mathfrak{C}} \Re_{0} / \mathscr{S}_{\mathfrak{E}}$, (b) follows from (a) if $\left|\Re_{0}\right|=3^{2}$. Suppose $\left|\mathfrak{R}_{0}\right|=3^{3}$. Let $\mathfrak{I}_{0}$ be the largest subgroup of $\mathfrak{I}$ whose elements induce automorphisms of $\mathfrak{S}_{\mathfrak{C}} \Re_{0} / \mathfrak{S}_{\mathfrak{L}} D\left(\Re_{0}\right)$ of determinant 1 . Thus, $\left|\mathfrak{I}: \mathfrak{I}_{0}\right| \leqq 2$, and so $\mathfrak{I}_{0} \supset \mathfrak{S}$. Choose $T \in \mathfrak{I}_{0}-\mathfrak{F}$ with $T^{2} \in \mathfrak{K}$. Thus, $T$ inverts $\mathfrak{S} \Re_{0} / \mathfrak{S} \boldsymbol{D}\left(\Re_{0}\right)$. It follows that every element of $\Re_{0}-\boldsymbol{D}\left(\Re_{0}\right)$ is $\mathfrak{M}$-real. Furthermore, there is $R \in \Re_{0}-\boldsymbol{D}\left(\Re_{0}\right)$ such that $\boldsymbol{C}_{\mathfrak{f}}(R)$ contains a four-group, so we are done by yet another application of Lemmas 14.21 and 14.22.

LEMmA 15.33. If $|\mathfrak{B}| \geqq 8$ and $\hat{\mathfrak{W}} \neq \varnothing$, then $\boldsymbol{C}\left(\mathfrak{B}_{0}\right) \subseteq \mathfrak{M}$ for every subgroup $\mathfrak{B}_{0}$ of $\mathfrak{B}$ of index at most 4 .

Proof. Suppose false, and $C\left(\mathfrak{B}_{0}\right) \nsubseteq \mathfrak{M},\left|\mathfrak{B}_{:} \mathfrak{B}_{0}\right| \leqq 4$. By Lemma 14.20, $C_{\mathfrak{R}}(\mathfrak{B})$ is cyclic. Choose $M \in \hat{\mathfrak{M}}$. Let $\mathfrak{B}_{1}=\mathfrak{B}_{0} \cap \mathfrak{B}_{0}^{\mathfrak{R}} \cap \mathfrak{B}_{0}^{\mathfrak{m}-1}$. If $\mathfrak{B}_{1} \neq 1$, we get $C\left(\mathfrak{B}_{0}\right) \subseteq C\left(\mathfrak{B}_{1}\right) \triangleleft N\left(\mathfrak{B}_{1}\right)$, and $\mathfrak{B}\langle M\rangle \subseteq N\left(\mathfrak{B}_{1}\right)$. Thus, $N\left(\mathfrak{B}_{1}\right) \subseteq \mathfrak{M}$, by Lemma 15.31. We conclude that $\mathfrak{B}_{1}=1$.

Since $\left|\mathfrak{B}: \mathfrak{B}_{0}\right|=2^{a} \leqq 4$, we have $\left|\mathfrak{B}: \mathfrak{B}_{1}\right|=|\mathfrak{B}| \leqq 2^{3 a} \leqq 64$. Hence, $|\mathfrak{B}|=2^{v}$ and $3 \leqq v \leqq 6$.

Suppose $\mathfrak{R}^{\prime}=1$. In this case, every element of $\mathfrak{R}$ is $\mathfrak{M}$-real, and so every element of $\boldsymbol{C}_{\mathfrak{R}}(\mathfrak{B})$ of order 3 is in $\hat{\mathfrak{M}}$. We conclude that $C_{\Re(}(\mathfrak{B})=1$. So $v=4,5$ or 6 . Since $v \leqq 6$, it follows that $|\Re|=3^{2}$, as $G L(6,2)$ has no abelian subgroup of type $\left(3^{2}, 3\right)$.

Suppose $C_{\mathfrak{s}}(\Re)=\mathfrak{B}^{0} \neq 1$. In this case, there is $R \in \mathfrak{R}^{\sharp}$ such that $\left|C_{\mathfrak{F}}(R)\right| \geqq 8$, and so $\mathfrak{B}_{0} \cap \mathfrak{B}_{0}^{R} \cap \mathfrak{B}_{0}^{R^{-1}} \neq 1$, as $C_{\mathfrak{s}_{0}}(R) \neq 1$. Since $R \in \hat{\mathfrak{M}}$, this is impossible. So $C_{\mathfrak{r}}(\Re)=1$. Hence, $v=4$ or 6 .

\section{Case 1. $v=6$.}

Write $\mathfrak{B}=\mathfrak{B}_{1} \times \mathfrak{B}_{2} \times \mathfrak{B}_{3}$, where $\mathfrak{B}_{i}$ is a four-group which admits $\Re$. Let $\Re^{i}=\left\langle R^{i}\right\rangle=\boldsymbol{C}_{\mathfrak{r}}\left(\mathfrak{B}_{i}\right)$. Since $\mathfrak{B}_{0} \cap \boldsymbol{C}\left(\mathfrak{R}^{i}\right)=1$, it follows that $\Re^{1}, \Re^{2}, \Re^{3}$ are all distinct, and $R^{i} \in \hat{\mathfrak{M}}$. Since $\mathfrak{B}_{0} \cap C\left(\mathfrak{R}^{i}\right)=1$, it follows that $\mathfrak{B}_{0} \cap \mathfrak{B}_{\sigma(1)} \mathfrak{B}_{\sigma(2)}$ is a four-group for every permutation $\sigma$ of $\{1,2,3\}$. By Lemma 5.31, each of these 4-groups is normalized by a subgroup of $\Re$ of order 3 . Since $\boldsymbol{C}\left(\mathfrak{B}_{0}\right) \nsubseteq \mathfrak{M}$, it follows that for each permutation $\sigma$ of $\{1,2,3\}, N\left(\mathfrak{B}_{0} \cap \mathfrak{B}_{\sigma(1)} \mathfrak{B}_{\sigma(2)}\right) \nsubseteq \mathfrak{M}$. Since $R^{i} \in \mathfrak{M} \hat{\mathbb{R}}$, Lemma 15.31 implies that $N_{\Re}\left(\mathfrak{B}_{0} \cap \mathfrak{B}_{\sigma(1)} \mathfrak{B}_{\sigma(2)}\right)=\Re^{4}$ is the remaining subgroup of $\Re$ of order 3. Since $\mathfrak{B}_{0}=\left(\mathfrak{B}_{0} \cap \mathfrak{B}_{1} \mathfrak{B}_{2}\right) \times\left(\mathfrak{B}_{0} \cap \mathfrak{B}_{1} \mathfrak{B}_{3}\right)$, it follows that $\mathfrak{R}^{4} \leqq N\left(\mathfrak{B}_{0}\right)$. 
Since $\mathfrak{S} / \mathscr{S}_{0} \times \mathfrak{R} \mathscr{S}_{0} / \mathscr{S}_{0}$ acts on $\mathfrak{B}$, it follows that $\mathfrak{S}$ normalizes $\mathfrak{B}_{i}$, $1 \leqq i \leqq 3$, and so $\mathfrak{S} \subseteq C(\mathfrak{B})$. Since $C_{\mathfrak{R}}(\mathfrak{B})=1$, it follows that $\mathfrak{F}$ is a $S_{2}$-subgroup of $\boldsymbol{C}_{\mathfrak{n}}\left(\mathfrak{B}_{0}\right)$. Since $N(\mathfrak{K}) \subseteq \mathfrak{M}$, it follows that $\mathfrak{K}$ is a $S_{2}$-subgroup of $C\left(\mathfrak{B}_{0}\right)$. If $C\left(\mathfrak{B}_{0}\right)$ is a $3^{\prime}$-group, then since $N(\mathfrak{X}) \subseteq \mathfrak{M}$ for every non identity characteristic subgroup $\mathfrak{Y}$ of $\mathfrak{S}_{\mathfrak{C}}$, we get $\boldsymbol{C}\left(\mathfrak{B}_{0}\right)=$ $\left(\boldsymbol{C}\left(\mathfrak{B}_{0}\right) \cap \boldsymbol{C}\left(\boldsymbol{Z}\left(\mathfrak{S}_{2}\right)\right)\right) \cdot\left(\boldsymbol{C}\left(\mathfrak{B}_{0}\right) \cap \boldsymbol{N}(\boldsymbol{J}(\mathfrak{\mathcal { E }}))\right) \subseteq \mathfrak{M} . \quad$ Hence, 3|| $\boldsymbol{C}\left(\mathfrak{B}_{0}\right) \mid . \quad$ Since $\mathfrak{R}^{4} \subseteq N\left(\mathfrak{V}_{0}\right)$, it follows that $N\left(\mathfrak{B}_{0}\right)$ contains a $S_{3}$-subgroup $\widetilde{\Re}$ of $\mathbb{S}$ with $\mathfrak{R}^{4} \subseteq \widetilde{\Re}$, and with $\widetilde{\mathfrak{R}}$ permutable with $\mathfrak{S}$. Also, $\left|\boldsymbol{C}\left(\mathfrak{B}_{0}\right): C\left(\mathfrak{B}_{0}\right) \subset \mathfrak{M}\right|=3$.

Since $\mathscr{H}$ is a $S_{2}$-subgroup of $\boldsymbol{C}\left(\mathfrak{B}_{0}\right)$ which is permutable with $\widetilde{\Re}$, it follows that $\mathfrak{S}$ is permutable with $\mathfrak{F}=\langle\widetilde{\mathfrak{R}}, \mathfrak{R}\rangle \leqq C\left(\mathfrak{R}^{4}\right)$. Let $\mathfrak{R}=\mathfrak{S} \mathfrak{F}$. Since $\mathfrak{F} \subseteq C\left(\Re^{4}\right), \mathfrak{F}$ is solvable. Since $\mathfrak{L}=\mathfrak{S} \mathfrak{F}$, so also $\mathbb{L}=\mathfrak{R}_{1} \mathfrak{F}$, where $\mathfrak{R}_{1}=\mathfrak{S}_{\mathfrak{C}} \mathfrak{R}^{4}$. Since

$$
\bigcap_{X \in \mathfrak{E}} \mathfrak{R}_{1}^{X}=\bigcap_{X \in \mathfrak{F}} \mathfrak{Z}_{1}^{X} \supseteqq \Re^{4}
$$

it follows that $\mathfrak{L}$ is solvable. By Lemma 14.20 , we have $\mathfrak{L} \subseteq \mathfrak{M}$. Thus, $3 \nmid\left|C\left(\mathfrak{B}_{0}\right): C\left(\mathfrak{B}_{0}\right) \cap \mathfrak{M}\right|$, so this case does not arise.

\section{Case 2. $v=4$.}

Let $\mathfrak{B}=\mathfrak{B}_{1} \times \mathfrak{B}_{2}$, where $\mathfrak{B}_{i}$ is a four-group which admits $\mathfrak{R}$. Let $\Re^{i}=\left\langle R^{i}\right\rangle=C_{\mathfrak{R}}\left(\mathfrak{B}_{i}\right)$. Thus, $R^{i} \in \widetilde{\mathfrak{M}}$, and so $\mathfrak{B}_{i} \cap \mathfrak{B}_{0}=1, i=1,2$. Let $\Re^{3}=\langle R\rangle=N_{\mathfrak{R}}\left(\mathfrak{B}_{0}\right)$. By Lemma 5.31, we get $\left|\mathfrak{R}^{3}\right|=3$. If $\mathfrak{S}$ is a $S_{2}$-subgroup of $C_{\mathfrak{m}}\left(\mathfrak{B}_{0}\right)$, we can repeat the argument of Case 1. Thus, we may assume that $\mathfrak{S}_{\mathcal{E}}$ is properly contained in a $S_{2}$-subgroup $\mathfrak{S}_{1}$ of $\boldsymbol{C}\left(\mathfrak{B}_{0}\right)$.

Let $\mathfrak{I}_{1}=N_{\mathfrak{x}}(\mathfrak{R}), \mathfrak{I}_{2}=\mathfrak{S} \cap \mathfrak{I}_{1}$. Since $\mathfrak{R}^{1}, \mathfrak{R}^{2}$ are the only subgroups of $\Re$ of order 3 which have fixed points on $\mathfrak{B}$, it follows that $\mathfrak{I}_{1} / \mathfrak{I}_{2} \cong \mathfrak{I} / \mathfrak{F}$ is isomorphic to a subgroup of a dihedral group of order 8 .

We argue that $R$ is real in $N_{\mathfrak{m}}\left(\mathfrak{S}_{0}\right)$. Namely, $\mathfrak{I}_{1}$ contains an element $T$ which inverts $\Re$. Also, $\mathfrak{B}_{i}=\left\langle V_{i 1}, V_{i 2}\right\rangle,(i=1,2)$, and

$$
\mathfrak{B}_{0}=\left\langle V_{11} V_{21}, V_{12} V_{22}\right\rangle,
$$

for suitable generators $V_{i j}$, and we may assume that $T$ centralizes $V_{11}$, since $T \in N\left(\Re^{1}\right)$. Let $\langle W\rangle=C_{\mathfrak{B}_{2}}(T)$, and choose $\widetilde{\Re} \in \Re^{1}$ such that $W^{\widetilde{R}}=V_{21}$. Then $\widetilde{T}=\widetilde{R}^{-1} T \widetilde{R}$ inverts $\Re$ and centralizes both $V_{11}$ and $V_{21}$. Thus, $\left[V_{12}, \widetilde{T}\right]=V_{11},\left[V_{22}, \widetilde{T}\right]=V_{21}$, and so $\widetilde{T} \in N\left(\mathfrak{B}_{0}\right)$. It follows that $\mathfrak{S}_{2}$ is of index 4 in some $S_{2}$-subgroup $\mathscr{F}_{2}$ of $N_{\mathfrak{m}}\left(\mathfrak{B}_{0}\right)$, where $\mathfrak{S}_{1} \subset \mathfrak{F}_{2}$.

Since $\mathfrak{R}^{3} \subseteq N\left(\mathfrak{B}_{0}\right)^{\prime}$, it follows that $N\left(\mathfrak{B}_{0}\right)=\mathfrak{N}=\mathfrak{R}_{2} \cdot C_{\mathfrak{R}}\left(\Re^{3}\right)$, where $\mathfrak{R}_{2}$ is a $S_{2}$-subgroup of $\mathfrak{R}$ which contains $\mathfrak{K}_{2}$. If $\mathfrak{S}_{2}=\mathfrak{R}_{2}$, then let $\widetilde{F}=\left\langle\boldsymbol{C}_{\mathfrak{R}}\left(\mathfrak{R}^{3}\right), \mathfrak{R}\right\rangle$, so that $\mathfrak{H}_{2} \mathfrak{F}=\mathfrak{R}$ is a group, as is $\mathfrak{\mathcal { E }}_{2} \mathfrak{R}^{3}=\mathfrak{R}_{1}$. As before, we get $\mathfrak{R} \subseteq \mathfrak{M}$. Since $\mathfrak{R} \subseteq \mathbb{R}$, we are done. So we may assume that $\mathscr{S}_{2} \subset \mathfrak{R}_{2}$. Since $\left|\mathfrak{F}_{2}: \mathfrak{S}\right|=4$, and $\left|\mathfrak{T}: \mathfrak{F}_{\mathcal{E}}\right| \leqq 8$, while $\mathfrak{T}$ is a $S_{2}$ - 
subgraup of $\left(\mathfrak{B}\right.$, it follows that $\left|\mathfrak{N}_{2}: \mathfrak{F}_{2}\right|=2$, and $\mathfrak{R}_{2}$ is a $S_{2}$-subgroup of $\mathbb{S}$, while $|\mathfrak{T} / \mathfrak{S}|=8$.

Let $\mathfrak{R}_{3}$ be a $S_{3}$-subgroup of $\mathfrak{N}$ which contains $\mathfrak{R}^{3}$ and is permutable with $\mathfrak{R}_{2}$. Let $\tilde{\mathfrak{R}}_{2}=\boldsymbol{O}_{2}\left(\mathfrak{R}_{2} \mathfrak{N}_{3}\right)$. Since $\mathfrak{S}_{1}$ centralizes $\tilde{\mathfrak{N}}_{2} \mathfrak{R}^{3} / \tilde{\mathfrak{N}}_{2}$, it follows that $\left|\mathfrak{S}_{1}: \mathscr{S}_{1} \cap \tilde{\mathfrak{N}}_{2}\right| \leqq 2$. If $\mathfrak{H}_{1} \subseteq \tilde{\mathfrak{N}}_{2}$, we get that $\left|\tilde{\mathfrak{R}}_{2}: \mathfrak{S}_{1}\right| \leqq 2$, since $\mathfrak{R}_{2} \subset \tilde{\mathfrak{N}}_{2}$, and $\left|\mathfrak{R}_{2}: \mathfrak{S}_{1}\right|=4$. Thus, in this case, we again get $\mathfrak{N}=$ $\mathfrak{S}_{2} \cdot C_{\mathfrak{R}}\left(\Re^{3}\right)$, and the above argument works. We may assume that $\left|\mathfrak{F}_{1}: \mathfrak{S}_{1} \cap \tilde{\mathfrak{R}}_{2}\right|=2$. Hence, $\quad \tilde{\mathfrak{N}}_{2} \cap \mathfrak{M}=\mathfrak{S}_{1} \cap \tilde{\mathfrak{N}}_{2}$, since $\mathfrak{R}_{2} \cap \mathfrak{M} \subseteq \mathfrak{S}_{1}=$ $\boldsymbol{O}_{2}\left(\mathfrak{S}_{2} \mathfrak{R}^{3}\right)$. If $\left[\tilde{\mathfrak{N}}_{2}, \mathfrak{R}^{3}\right] \subseteq \mathfrak{S}_{1} \cap \tilde{\mathfrak{N}}_{2}$, then $\mathfrak{R}=\mathfrak{S}_{2} \cdot \boldsymbol{C}_{\mathfrak{R}}\left(\mathfrak{R}^{3}\right)$, and we are done. Hence, $\left|\tilde{\mathfrak{N}}_{2}: \mathfrak{H}_{1} \cap \tilde{\mathfrak{N}}_{2}\right| \geqq 4$. Since $\left|\mathfrak{N}_{2}: \tilde{\mathfrak{N}}_{2}\right| \geqq 2$, and $\left|\mathfrak{T}: \tilde{\mathfrak{S}}_{1}\right|=4$, while $\left|\mathfrak{F}_{1}: \mathfrak{S}_{1} \cap \tilde{\mathfrak{R}}_{2}\right|=2$, and since $\mathfrak{I}, \mathfrak{R}_{2}$ and $S_{2}$-subgroups of (5), we have $\left|\mathfrak{R}_{2}: \mathfrak{K}_{1} \cap \tilde{\mathfrak{R}}_{2}\right| \leqq 8$, whence we get $\left|\mathfrak{R}_{2}: \tilde{\mathfrak{N}}_{2}\right|=2$, $\left|\tilde{\mathfrak{R}}_{2}: \mathfrak{S}_{1} \cap \tilde{\mathfrak{N}}_{2}\right|=4$.

On the other hand, every element of $\mathscr{S}_{2}-\mathscr{S}_{1}$ inverts $\mathfrak{S}_{2} \mathfrak{R}^{3} / \mathscr{S}_{0}$, and so $\tilde{S}_{2} \cap \tilde{\mathfrak{R}}_{2}=\tilde{\mathfrak{S}}_{1} \cap \tilde{\mathfrak{N}}_{2}$, whence $\tilde{\mathcal{S}}_{2} / \mathscr{\mathcal { S }}_{2} \cap \tilde{\mathfrak{R}}_{2}$ is of order 4 . Since $\tilde{S}_{2} / \mathscr{S}_{2} \cap$ $\tilde{\mathfrak{N}}_{2} \cong \mathfrak{S}_{2} \tilde{\mathfrak{N}}_{2} / \tilde{\mathfrak{A}}_{2} \cong \mathfrak{R}_{2} / \tilde{\mathfrak{N}}_{2}$, we get $\left|\mathfrak{N}_{2}: \tilde{\mathfrak{N}}_{2}\right| \geqq 4$. This contradiction shows that this case does not arise.

It remains to treat the case $\Re^{\prime} \neq 1$.

Case $1 . \Re$ acts faithfully on $\mathfrak{B}$.

In this case, we get $v=6$ and $\Re$ is of order $3^{3}$ and exponent 3 . Thus, every non central element of $\Re$ is in $\hat{\mathfrak{M}}$.

Now $\Re$ has precisely 12 non central subgroups of order 3 , say $\Re^{1}, \cdots, \Re^{12}$, and $\left|C_{\mathfrak{s}}\left(\Re^{i}\right)\right|=4$. Let $V(i)=C_{\mathfrak{s}}\left(\Re^{i}\right)^{\sharp}$. Then $V(i) \cap V(j)=\varnothing$, if $i \neq j$, and so

$$
\widetilde{V}=\bigcup_{i=1}^{12} V(i)
$$

has cardinal 36 .

Also, $\left|\left[\mathfrak{B}, \Re^{i}\right]\right|=2^{4}$, and $\left[\mathfrak{B}, \Re^{i}\right]=\left[\mathfrak{B}, \Re^{i}\right] \cap C\left(R^{i} Z\right)_{1} \times\left[\mathfrak{B}, \Re^{i}\right] \cap C\left(R^{i} Z^{-1}\right)$, where $\Re_{\tau}=\left\langle R^{1}\right\rangle$, and $\langle Z\rangle=\mathfrak{R}^{\prime}$. Hence, $\left[\mathfrak{B}, \Re^{i}\right] \cap \mathfrak{B}_{0}$ is a four-group with admits 3 , by Lemma 5.31, together with the fact that $\left[\mathfrak{B}, \Re^{i}\right] \cap \mathfrak{B}_{0}$ does not admit any of the non central elements of $\Re$, by Lemma 15.31. Since $\bigcap_{i=1}^{12}\left[\mathfrak{B}, \mathfrak{R}^{i}\right]=1$, it follows that $Z$ normalizes $\mathfrak{B}_{0}$.

Set $R=R^{1}$. Now $\left|\mathfrak{B}_{0} \cap \mathfrak{B}_{0}^{R}\right| \leqq 4$, since $\mathfrak{B}_{0} \cap \mathfrak{B}_{0}^{R} \cap \mathfrak{B}_{0}^{R^{-1}}=1$. Hence, $\left|\mathfrak{B}_{0} \cap \mathfrak{B}_{0}^{R}\right|=4$. Hence, $\left|\mathfrak{B}_{0}^{\sharp} \cup \mathfrak{B}_{0}^{\sharp R}\right|=27$, and so

$$
\mathfrak{B}_{0}^{\sharp} \cup \mathfrak{B}_{0}^{\ddagger R} \cup \tilde{\mathfrak{B}}=\mathfrak{B}^{\sharp} \text {. }
$$

This implies that $\mathfrak{B}_{0}^{R^{-1}} \cong \mathfrak{B}_{0} \cup \mathfrak{B}_{0}^{R}$. This is false, since

$$
\left|\mathfrak{B}_{0}^{\sharp} \cup \mathfrak{B}_{0}^{\sharp R} \cup \mathfrak{B}_{0}^{\sharp R-1}\right|=3.15-3.3=36, \quad\left|\mathfrak{B}_{0}^{\sharp} \cup \mathfrak{B}_{0}^{\sharp R}\right|=27 .
$$

Case 2. $\mathfrak{R}$ does not act faithfully on $\mathfrak{B}$. 
Let $\widetilde{\mathfrak{R}}=\boldsymbol{C}_{\mathfrak{R}}(\mathfrak{B})$. Then $\widetilde{\mathfrak{R}} \triangleleft \Re$ and $\Re$ is cyclic. Since $\widetilde{\mathfrak{R}}$ and $\boldsymbol{O}_{3^{\prime}, 3}\left(\boldsymbol{C}_{\mathfrak{M}}(\mathfrak{B})\right) / \boldsymbol{O}_{3^{\prime}}\left(\boldsymbol{C}_{\mathfrak{M}}(\mathfrak{B})\right)$ are incident, and since $\mathfrak{R} \subseteq \mathfrak{M}^{\prime}$, we get $\widetilde{\mathfrak{R}} \subseteq \boldsymbol{Z}(\mathfrak{R})$. Since $\widetilde{\mathfrak{R}} \cap \widehat{\mathfrak{M}}=\varnothing$, it follows that $\mathfrak{T}$ centralizes $\widetilde{\mathfrak{R}} \mathfrak{Y}_{0} / \mathfrak{S}_{0}$. Since $\mathfrak{R} \subseteq \mathfrak{M}^{\prime}$, it follows that $\mathfrak{I}$ has no fixed points on $\widetilde{R}_{0} \mathfrak{S}_{0} / \mathfrak{R}_{0}^{\prime} \mathfrak{S}_{0}$, and so $\widetilde{\mathfrak{R}} \subseteq \mathfrak{R}^{\prime}$.

Suppose $\subseteq$ is a subgroup of $\Re$ of exponent 3 and order 27 . Then $\widetilde{\Re} \cap \mathfrak{S}=\mathfrak{S}^{\prime}$. By Lemma 5.31, it follows that $\mathfrak{S}$ contains a subgroup $\mathfrak{B}$ of order $3^{2}$ which normalizes a non trivial subgroup $\mathfrak{B}_{00}$ of $\mathfrak{B}_{0}$. Thus, $C\left(\mathfrak{B}_{0}\right) \subseteq N\left(\mathfrak{B}_{00}\right), \mathfrak{S} \mathfrak{B} \cong N\left(\mathfrak{B}_{00}\right)$, and so $C\left(\mathfrak{B}_{0}\right) \subseteq \mathfrak{M}$, by Lemma 14.20. We conclude that no such $\subseteq$ exists. By a result of Blackburn [6], $\Re$ is of maximal class, and so $|\widetilde{\Re}|=3$, and $|\Re| \geqq 3^{4}$. If $|\Re| \geqq 3^{5}$, then since $v \leqq 6$, we get $\Re / \widetilde{\Re} \cong Z_{3} S Z_{3}$. If $\mathscr{S} / \Re$ is the unique elementary subgroup of $\Re / \widetilde{\Re}$ of order 27 , then $\Omega_{1}(\mathfrak{S})$ is of exponent 3 and order at least 27. This is false, and so $|\Re|=3^{4}$. Since $\widetilde{\Re}=\langle Z\rangle$, where $Z$ is non real in $\mathfrak{M}$, it follows that no element of $\mathfrak{T}$ inverts $\Re_{0} \mathfrak{S}_{0} / \mathfrak{S}_{0} \Re_{0}{ }^{\prime}$, and so $|\mathfrak{T} / \mathfrak{L}|=2$, and $\mathfrak{I}=\langle\mathfrak{S}, T\rangle$ where $T$ inverts $\mathfrak{R} / \mathfrak{R}^{\prime}$. Thus, $T$ centralizes $\mathfrak{S}_{0} \Re^{\prime} / \mathfrak{S}_{0}\left[\Re^{\prime}, \Re\right]$, and so $T$ inverts $\mathfrak{S}_{0}\left[\Re^{\prime}, \Re\right] / \mathscr{S}_{0}\left[\Re^{\prime}, \Re, \Re\right]$. Since $\widetilde{\Re}=\left[\Re^{\prime}, \Re\right]$, this is the desired contradiction. The proof is complete.

LEMmA 15.34. If $|\mathfrak{B}| \geqq 8$, then $C\left(\mathfrak{B}_{0}\right) \subseteq \mathfrak{M}$ for every subgroup $\mathfrak{V}_{0}$ of $\mathfrak{B}$ of index at most 4 .

Proof. Suppose false, and $C\left(\mathfrak{B}_{0}\right) \nsubseteq \mathfrak{M},\left|\mathfrak{B}: \mathfrak{B}_{0}\right| \leqq 4$. Then by Lemma $14.20, C_{\Re}(\mathfrak{B})$ is cyclic. By Lemma 15.33, $\hat{\mathfrak{M}}=\varnothing . \quad$ By Lemma $15.32(\mathrm{~b}),|\mathfrak{I} / \mathfrak{S}|=2$. Hence, $l_{3}(\mathfrak{M})=1$, so that $\Re=\Re_{1}$. Since $\Re \subseteq \mathfrak{M}^{\prime}$, we have $\mathfrak{I}=\langle\mathfrak{S}, T\rangle$, where $T$ inverts $\Re / \Re^{\prime}$. By Lemma 15.32 (a), $\Re^{\prime} \neq 1$.

Suppose $\left|\Omega_{1}(\Re)\right|=3^{3}$. By Lemma 15.32 (a), $T$ centralizes $\mathscr{S}_{0} \Omega_{1}(\Re)^{\prime} / \mathscr{Y}_{0}$, and so $T$ inverts $\Omega_{1}(\Re) / \Omega_{1}(\Re)^{\prime}$. If $\Omega_{1}(\Re)^{\prime}$ centralizes $\mathfrak{N}$, then $\mathfrak{B}_{0}$ contains a non identity subgroup $\mathfrak{B}_{00}$ which is normalized by a subgroup of $\Omega_{1}(\Re)$ of order 9 , against Lemma 14.20 . If $\left[\Omega_{1}(\Re)^{\prime}, \mathfrak{B}\right] \neq 1$, then every element of $\Omega_{1}(\Re)-\Omega_{1}(\Re)^{\prime}$ is in $\hat{\mathfrak{M}}$, against $\hat{\mathfrak{M}}=\varnothing$. So $\left|\Omega_{1}(\Re)\right| \neq 3^{3}$.

By a result of Blackburn [6], $\Re$ is of maximal class. We can choose $R \in \mathfrak{R}_{1}^{*}$ such that $R^{T}=R^{-1}$. Since $R \notin \hat{\mathfrak{M}}$, it follows that $\left|C_{\mathfrak{B}}(R)\right| \leqq 2$, and so $C_{\mathfrak{B}}(R)=C_{\mathfrak{B}}\left(\Re_{0}\right)$.

First, suppose $R \notin Z(\Re)$. In this case, $C_{\Re_{1}}(T)=\langle Z\rangle \subseteq Z(\mathfrak{I})$, and all elements of $\Re_{0}-\langle Z\rangle$ are $\mathfrak{M}$-real. Hence, $\boldsymbol{C}_{\mathfrak{B}}(R)=C_{\mathfrak{B}}\left(R Z^{i}\right)=C_{\mathfrak{B}}\left(\Re_{1}\right)$ for all $i$, and so $Z$ centralizes $\mathfrak{B}$. Let $\mathfrak{B}^{*}=[\mathfrak{B}, \mathfrak{R}], \mathfrak{B}_{0}^{*}=\mathfrak{B}_{0} \cap \mathfrak{B}^{*}$. Then $\langle Z, R\rangle$ normalizes $\mathfrak{B}_{0}^{*} \cap \mathfrak{B}_{0}^{* R}$, and so by Lemma $14.20, \mathfrak{B}_{0}^{*} \cap$

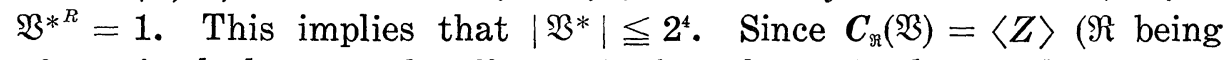
of maximal class, so that $\Re_{0} /\langle Z\rangle$ is the only minimal normal subgroup of $\Re /\langle Z\rangle$ ), it follows that $|\Re| \leqq 3^{3}$, which is false. Hence, $\langle R\rangle=Z(\mathfrak{I})$, and so $C_{\Re}(\mathfrak{B})=1$. 
Since $|\Re| \geqq 3^{4}, \Re_{0}$ is contained in an abelian subgroup $\mathfrak{A}$ of $\Re$ of order 27 such that $\mathfrak{A} \triangleleft\langle\mathfrak{R}, T\rangle$. Thus, $\sigma^{1}(\mathfrak{A}) \triangleleft \Re$, so $\mathscr{J}^{1}(\mathfrak{A})=\langle R\rangle$. Since $\mathfrak{N}$ acts faithfully on $\mathfrak{B}$, it follows that if $A \in \mathfrak{A}-\mathfrak{R}$, and $A^{3}=1$, then $\left|\boldsymbol{C}_{\mathfrak{B}}(A)\right| \geqq 2^{6}$. Again, let $\mathfrak{B}^{*}=[\mathfrak{B}, \mathfrak{R}], \mathfrak{B}_{0}^{*}=\mathfrak{B}_{0} \cap \mathfrak{B}^{*}$. Then $\mathfrak{B}_{0}^{*} \cap \mathfrak{B}_{0}^{* R}=\mathfrak{W}$ admits $R$ and is of index at most $2^{4}$ in $\mathfrak{B}^{*}$. Hence, $\mathfrak{W} \cap C(A)=\mathfrak{W}_{0} \neq 1$. Since $\langle A, R\rangle=\mathfrak{R}_{0}$ normalizes $\mathfrak{W}_{0} \subseteq \mathfrak{B}_{0}$, the proof is completed by a final application of Lemma 14.20.

We now turn to the control of the elements of $\mathfrak{M}$ of prime order $p \geqq 5$. This is easy.

Lemma 15.35. Suppose $\mathfrak{P}=\langle P\rangle \subseteq \mathfrak{M},|\mathfrak{P}|=p, p$ is a prime $\geqq 5$, $P$ is $\mathfrak{M}$-real, and $C_{\mathfrak{5}_{0}}(\mathfrak{P})$ contains an elementary subgroup (5 of order 8. Then $\mathbb{F} \in \mathscr{M}^{*}$.

Proof. Since $S_{p}$-subgroups of $\mathfrak{M}$ are cyclic, we may assume that $\mathfrak{T P}$ is a group, and that $\mathfrak{P} \subseteq \mathfrak{D}$. Let $\mathfrak{I}_{0}=\boldsymbol{O}_{2}(\mathfrak{T} \mathfrak{P})$. Then $\mathfrak{I}_{0} \in N_{\mathfrak{m}}^{*}(\mathfrak{P} ; 2)$, and $P$ is real in $N_{\mathscr{M}}\left(\mathfrak{I}_{0}\right)$. In particular, $\mathfrak{I}_{0} \subset \mathfrak{I}$.

Since $\Re \cdot C_{\mathfrak{F}_{0}}(\mathfrak{P}) \subseteq N(\mathfrak{P})$, Lemma 15.29 implies that $N(\mathfrak{P}) \subseteq \mathfrak{M}$. The remainder of the proof follows the lines of Lemma 15.31.

Lemma 15.36 If $\mathfrak{U}$ is an elementary abelian 2-subgroup of $\overline{\mathfrak{M}}=\mathfrak{M} / \boldsymbol{O}_{3^{\prime}}(\mathfrak{M})$, then $\mathfrak{U}$ normalizes some $S_{3^{-}}$-subgroup of $\overline{\mathfrak{M}}$.

Proof. If $\overline{\mathfrak{M}}$ is 3 -closed, the lemma is trivial. We may assume that $l_{3}(\overline{\mathfrak{M}}) \geqq 2$. Thus, $\overline{\mathfrak{M}} / / O_{3^{\prime}}(\overline{\mathfrak{M}})$ is isomorphic to a subgroup of $G L(2,3)$ which is 3-reduced, has order divisible by 3 , and has no normal subgroup of index 3. Hence, $\overline{\mathfrak{M}} / \boldsymbol{O}_{3}(\overline{\mathfrak{M}}) \cong G L(2,3)$. The image of $\mathfrak{U}$ in $\overline{\mathfrak{M}} / \boldsymbol{O}_{3}(\overline{\mathfrak{M}})$ thus normalizes a $S_{3}$-subgroup of $\overline{\mathfrak{M}} / \boldsymbol{O}_{3}(\overline{\mathfrak{M}})$, and the lemma follows.

Once again, let $\mathscr{J}=\left\{I \mid I\right.$ is an involution of $\left.\mathfrak{M}, C_{\mathfrak{n}}(I) \in \mathscr{C}^{*}\right\}$.

LEMmA 15.37. Suppose $I$ is an involution of $\mathfrak{M}$ and $\boldsymbol{C}_{\mathfrak{B}}(I)=\mathfrak{B}_{0}$ is a hyperplane of $\mathfrak{B}$. Then $[\mathfrak{B}, I]=\langle J\rangle$, where $J \in \mathscr{F}$.

Proof. By the preceding lemma, we may assume that $I \in \mathfrak{I} \cap$ $\boldsymbol{N}\left(\boldsymbol{O}_{3^{\prime}}(\mathfrak{M}) \Re\right)$. Since $\mathfrak{K}_{0} \Re$ char $\boldsymbol{O}_{3^{\prime}}(\mathfrak{M}) \Re$, we get $I \in N\left(\mathfrak{K}_{0} \Re\right)$. Let $\mathfrak{Y}=$ $\mathfrak{S}_{0} \Re / \mathfrak{K}_{0} \cong \Re$. Let $\mathfrak{Y}_{0}=C_{\mathfrak{y}}(\mathfrak{V}), \mathfrak{Y}_{1}=[\mathfrak{Y}, I], \mathfrak{Y}_{2}=C_{\mathfrak{y}}(I)$. If $\mathfrak{Y}_{0}$ is non cyclic, then $\mathfrak{B}^{\sharp} \subseteq \mathscr{J}$, Lemma 14.22, and we are done. We may assume that $\mathfrak{Y}_{0}$ is cyclic. Since $\mathfrak{B}$ is 2-reducible in $\mathfrak{M}, \mathfrak{Y}_{1} \nsubseteq \mathfrak{Y}_{0}$, and since $\left|\mathfrak{B}: \mathfrak{B}_{0}\right|=2$, we get $\left|\mathfrak{Y}_{1}: \mathfrak{Y}_{1} \cap \mathfrak{Y}_{0}\right|=3$, and $\left[\mathfrak{B}, \mathfrak{Y}_{1}\right]=\mathfrak{B}^{0}$ is a four-group which admits $\mathfrak{Y}$ and contains $J$. Let $\tilde{\mathfrak{Y}}=\boldsymbol{C}_{\mathfrak{y}}\left(\mathfrak{B}^{\circ}\right)$. Then $|\mathfrak{Y}: \tilde{\mathfrak{Y}}|=3$. Since $J \in \mathfrak{B}^{0}$, we are done by Lemma 14.22 if $\tilde{\mathfrak{Y}}$ is non cyclic. We may assume that $\tilde{\mathfrak{V}}$ is cyclic. Thus, $\mathfrak{R}^{\prime}=1$, and so every element of $\mathfrak{R}$ is $\mathfrak{M}$-real. Thus, if $Y \in \mathfrak{R}^{\sharp}, Y^{3}=1$, and $Y \mathfrak{S}_{0} \in \tilde{\mathfrak{V}}$, then $Y \in \mathfrak{W}$, so we are done by Lemma 15.31. 
Lemma 15.38. One of the following holds:

(a) $\mathfrak{B}^{\sharp} \subseteq \mathscr{J}$. M-real.

(b) $\boldsymbol{C}(\mathfrak{B})$ contains no elements of odd prime order which are

Proof. If $|\mathfrak{B}|=2$, then obviously $\mathfrak{B}^{\sharp} \subseteq \mathscr{J}$. If $|\mathfrak{B}|=4$, then $\left|\Re: C_{\Re}(\mathfrak{B})\right| \leqq 3$. If $C_{\Re}(\mathfrak{B})$ is non cyclic, then (a) holds by Lemma 14.22. If $C_{\mathfrak{r}}(\mathfrak{B})$ is cyclic, then $\mathfrak{R}^{\prime}=1$, and $\boldsymbol{C}_{\mathfrak{r}}(\mathfrak{B}) \cap \mathfrak{\mathfrak { N }} \neq \varnothing$, and we are done by Lemma 15.31. We may assume that $|\mathfrak{B}| \geqq 8$, and that $\boldsymbol{C}_{\mathfrak{r}}(\mathfrak{B})$ is cyclic. If $X$ is an element of $C(\mathfrak{B})$ of odd prime order which is $\mathfrak{M}$-real, then by Lemmas 15.31 and 15.35 , we get $\mathfrak{B}\langle X\rangle \in \mathscr{M}^{*}$, so (a) holds. The proof is complete.

Lemma 15.39. $\quad V\left(\operatorname{ccl}_{\mathfrak{G}}(\mathfrak{B}) ; \mathfrak{T}\right) \subseteq \boldsymbol{C}(\mathfrak{B})$.

Proof. We have built up enough information to copy the proof of Lemma 13.7.

LEMma 15.40. One of the following holds:

(a) $\mathfrak{B}^{\sharp} \subseteq \mathscr{J}$.

(b) $\quad V\left(\operatorname{ccl}_{\mathfrak{\Theta}}(\mathfrak{B}) ; \mathfrak{T}\right) \subseteq \mathfrak{S}_{\mathcal{J}}$ •

Proof. If (b) fails, then by Lemma 15.39, $\boldsymbol{C}(\mathfrak{B})$ is not 2-closed, and so (a) holds by Lemma 15.38 .

LEMma 15.41. Suppose $\mathfrak{A}$ is a subgroup of $\mathfrak{M} / \boldsymbol{C}(\mathfrak{B})$ of order 3, and $[\mathfrak{B}, \mathfrak{X}]$ is a four-group. Then $[\mathfrak{B}, \mathfrak{X}]^{*} \subseteq \mathscr{J}$.

Proof. We may assume that $\boldsymbol{C}(\mathfrak{B})$ is 2-closed, by Lemma 15.38 . Thus, $\mathfrak{S}_{0}$ is the $S_{2}$-subgroup of $C(\mathfrak{B})$. Let $\mathfrak{B}$ be a $S_{3}$-subgroup of $\boldsymbol{C}_{\mathfrak{M}\left(C_{(\mathfrak{B})}\right)}(\mathfrak{L})$, and let $\mathfrak{S}$ be a 3-subgroup of $\mathfrak{M}$ which maps onto $\mathfrak{B}$. Let $\mathfrak{W}=$ $[\mathfrak{B}, \mathfrak{2}]$, and let $\mathfrak{\Im}_{0}=\boldsymbol{C}_{\mathfrak{\Im}}(\mathfrak{B})$, so that $\left|\mathfrak{S}_{:} \mathfrak{S}_{0}\right|=3$. If $\mathfrak{S}_{0}$ is non cyclic, we are done, by Lemma 14.22. Suppose $\widetilde{S}_{0}$ is cyclic. If $\mathfrak{S}_{0} \cap \hat{\mathfrak{M}} \neq \varnothing$, we are done by Lemma 15.31, so suppose $\widetilde{S}_{0} \cap \hat{\mathfrak{M}}=\varnothing$. If $\subseteq$ is a $S_{3}$-subgroup of $\mathfrak{M}$, then since $\subseteq$ is metacyclic, we get $\mathfrak{R}^{\prime}=1$, and so $\widetilde{S}_{0} \cap \hat{\mathfrak{M}} \neq \varnothing$. We may therefore assume that $\subseteq$ is a proper subgroup of $\Re$. Let $\mathfrak{S}_{1}=\boldsymbol{N}_{\Re}(\mathfrak{S})$. It follows that $\mathfrak{S} / \boldsymbol{C}_{\mathfrak{\Im}}(\mathfrak{B})$ has at least 3 subgroups of order 3 whose fixed point subgroup on $\mathfrak{B}$ is of index 4 . This is impossible, since $\mathfrak{S} / \boldsymbol{C}_{\mathfrak{S}}(\mathfrak{B})$ is metacyclic. The proof is complete.

It is now straightforward to follow $\S 13$, and get that $|\mathfrak{B}| \leqq 4$.

By Theorems 13.5, 13.6, and 13.7, $\mathfrak{M}$ contains a non cyclic elementary abelian normal 2-subgroup $\mathfrak{H}$. Suppose $\mathfrak{H}$ is a four-group. Let $\widetilde{\mathfrak{R}}=C_{\mathfrak{R}}(\mathfrak{U})$. If $\widetilde{\mathfrak{R}} \subset \mathfrak{R}$, then $\mathfrak{U}$ is 2-reducible in $\mathfrak{M}$, and so $\mathfrak{U}=\mathfrak{B}$, 
whence $\mathfrak{U}^{\sharp} \subseteq \mathscr{J}$, by Lemma 15.38. If $\widetilde{R}=\mathfrak{R}$, then $\mathfrak{U}^{\sharp} \subseteq \mathscr{I}$, by Theorem 14.21. Now Theorem 13.4 implies than $\mathfrak{M}$ contains a normal elementary abelian 2-subgroup of order $\geqq 8$. Let $\mathscr{F}_{0}=\{\mathfrak{F}|\mathfrak{F} \triangleleft \mathfrak{M},| \mathfrak{F} \mid \geqq 8$, $\widetilde{F}$ is elementary abelian\}, and let $\mathscr{F}$ be the set of minimal elements of $\mathscr{F}_{0}$. Let $\mathscr{F}^{*}=\{\mathfrak{F} \in \mathscr{F} \mid \boldsymbol{C}(\mathfrak{F})$ is not a 2 -group $\}$. If $\mathscr{F}^{*} \neq \varnothing$, choose $\mathfrak{F} \in \mathscr{F}^{*}$; otherwise, choose $\mathfrak{F} \in \mathscr{F}^{*}$. Let $\mathfrak{F} / \mathbb{F}$ be a chief factor of $\mathfrak{M}$.

Lemma 15.43. Suppose $\mathfrak{P}$ is of order $3, \mathfrak{P} \subseteq \boldsymbol{C}(\mathfrak{F}) \cap \boldsymbol{O}_{3^{\prime}, 3}(\mathfrak{M})$, and $\boldsymbol{C}_{\mathfrak{F}}(\mathfrak{P})$ contains a four-group. If $\mathbb{R} \in \boldsymbol{U}_{\mathfrak{N}}^{*}(\mathfrak{P} ; 2)$, then $\boldsymbol{N}(\mathbb{R}) \subseteq \mathfrak{M}$.

Proof. We assume without loss of generality that $\mathbb{Q} \subseteq \mathfrak{I}, \mathfrak{P} \subseteq \Re$. Thus, $\mathfrak{R} / \mathfrak{C}_{0}$ is a $S_{2}$-subgroup of $C_{\mathfrak{M} \mathfrak{E}_{0}}(\mathfrak{P})$. Let $\Re_{2}=\Re_{1} \cap C(\mathfrak{F})$. Since $\mathfrak{F} \cap \mathfrak{B} \neq 1$, it follows that $\Re_{2}$ centralizes $\mathfrak{B}$. Also, $\mathfrak{S}_{0} \Re_{2} \triangleleft \mathfrak{M}$, and $\mathfrak{P} \subseteq \mathfrak{H}_{\mathbb{C}} \Re_{2}$. Since $\mathfrak{H}_{0} \Re_{2} \triangleleft \mathfrak{M}$, so also $\left[Z\left(\mathscr{S}_{0}\right), \mathfrak{R}_{2}\right]=\mathfrak{W} \triangleleft \mathfrak{M}$. If $\mathfrak{W} \neq 1$, then $\mathfrak{W} \cap \mathfrak{B} \neq 1$, and so $\mathfrak{W} \supseteqq \mathfrak{B}$, as $C_{\mathfrak{g B}}\left(\Re_{2}\right)=1$. This is false, since $\mathfrak{R}_{2} \subseteq C(\mathfrak{B})$. So $\mathfrak{W}=1$. Clearly, $\mathbb{Q} \supseteqq \mathfrak{S}_{0}$, and since $\boldsymbol{O}_{2^{\prime}}(\mathfrak{M})=1$, we have $\boldsymbol{Z}(\mathfrak{Q}) \subseteq \boldsymbol{Z}\left(\mathfrak{S}_{0}\right)$. Hence, [P, $\left.\boldsymbol{Z}(\mathfrak{Q})\right]=1$.

Since $\boldsymbol{C}_{\widetilde{F}}(\mathfrak{Z})$ contains a four-group, Lemmas 13.20 and 14.22 imply that $N(\mathfrak{P}) \subseteq \mathfrak{M}$. Let $\mathfrak{R}=N(\mathbb{R}), \mathfrak{S}=\boldsymbol{O}_{3^{\prime}}(\mathfrak{R})$. If $S_{3}$-subgroups of $\mathfrak{R} \cap \mathfrak{M}$ are non cyclic, then $\mathfrak{R} \subseteq \mathfrak{M}$ by Lemma 15.29. We may assume that $S_{3}$-subgroups of $\mathfrak{R} \cap \mathfrak{M}$ are cyclic. Since $\boldsymbol{C}(\mathfrak{P}) \subseteq \mathfrak{M}, S_{3}$-subgroups of $\mathfrak{R}$ are cyclic. Let $\mathfrak{Q}_{2}$ be a $S_{2}$-subgroup of $\mathfrak{S}$ which admits $\mathfrak{P}$. Since $\mathfrak{R} \in \boldsymbol{U}_{\mathfrak{M} R}^{*}(\mathfrak{P} ; 2)$, and $N(\mathfrak{P}) \subseteq \mathfrak{M}$, it follows that $C_{\mathfrak{R}_{2} \backslash \mathfrak{R}}(\mathfrak{P})=1$, so that $\mathfrak{R}_{2}=\mathfrak{R}\left[\mathbb{L}_{2}, \mathfrak{P}\right]$. Since $\mathfrak{P}$ centralizes $Z(\mathbb{R})$, so does $\mathfrak{\Omega}_{2}$. Since $Z(\mathfrak{I}) \subseteq Z(\mathbb{R})$, and since $\mathfrak{S}^{\sharp} \subseteq \mathscr{F}$, while $\Omega_{1}(\boldsymbol{Z}(\mathfrak{T})) \subseteq \mathfrak{B}$, it follows that $\mathfrak{\Omega}_{2} \subseteq \mathfrak{M}$, whence $\mathfrak{R}_{2}=\mathfrak{R}$. If $\mathfrak{P} \subseteq \mathfrak{N}^{\prime}$, we get $\mathfrak{R}=\mathfrak{R} \cdot \boldsymbol{N}_{\mathfrak{N}}(\mathfrak{P}) \subseteq \mathfrak{M}$. So we may assume that $\mathfrak{P} \nsubseteq \mathfrak{R}^{\prime}$. Hence, $\mathfrak{N}$ has a normal 3 -complement, and so $\mathbb{R}$ is a $S_{2}$-subgroup of $\mathfrak{M}$, whence $\mathbb{Z}=\mathfrak{T}$. So $\mathfrak{N} \subseteq \mathfrak{M}$ by Lemma 15.42 .

LEMMA 15.44. Suppose $\mathfrak{P}$ is of order $3, \mathfrak{P} \subseteq \boldsymbol{C}(\mathfrak{F}) \cap \boldsymbol{O}_{3^{\prime}, 3}(\mathfrak{M})$, and $\boldsymbol{C}_{\widetilde{F}}(\mathfrak{P})$ contains a four-group. Then $\mathfrak{F} \mathfrak{P} \in \mathscr{L}^{*}$.

Proof. Let $\mathscr{S}=\{\mathfrak{S} \mid \mathfrak{F} \mathfrak{S} \subseteq \subseteq \subseteq \mathbb{S}, \subseteq$ is solvable, $\subseteq \nsubseteq \mathfrak{M}\}$, and suppose that $\mathscr{S} \neq \varnothing$. By Lemma 15.29 , if $\mathfrak{S}_{0} \in \mathscr{S}$, then $S_{3}$-subgroups of $\mathfrak{S}_{0} \cap \mathfrak{M}$ are cyclic. By Lemmas 14.20 and 14.22, N(P) $\subseteq \mathfrak{M}$, and so if $\mathfrak{S}_{0} \in \mathscr{S}, S_{3}$-subgroups of $\mathfrak{S}_{0}$ are cyclic.

Choose $\mathscr{S} \in \mathscr{S}$ with $|\mathfrak{S} \cap \mathfrak{M}|_{2}$ maximal; with this restriction, maximize $|\mathfrak{S}|_{2}$, and with these restrictions, minimize $\mathfrak{S}$. Let $\mathfrak{S}_{0}=\boldsymbol{O}_{3^{\prime}}(\mathfrak{S})$, and let $\widetilde{S}_{2}$ be a $S_{2}$-subgroup of $\subseteq$ which contains a $S_{2}$-subgroup $\widetilde{\Im}_{2}$ of

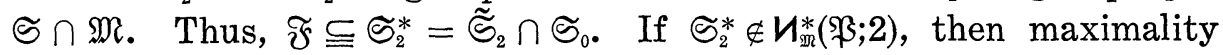
of $|\subseteq \cap \mathfrak{M}|_{2}$ implies that $\mathfrak{N}(\mathfrak{Y}) \subseteq \mathfrak{M}$ for every non identity characteristic subgroup $\mathfrak{Y}$ of $\mathfrak{S}_{2}^{*}$. Hence, $\mathfrak{S}_{2}^{*}$ is a $S_{2}$-subgroup of $\mathfrak{S}_{0}$. Since $\mathfrak{S}_{0}=\left(\mathfrak{S}_{0} \cap \boldsymbol{C}\left(\boldsymbol{Z}\left(\mathfrak{S}_{2}^{*}\right)\right)\right)\left(\mathfrak{S}_{0} \cap \boldsymbol{N}\left(\boldsymbol{J}\left(\mathfrak{S}_{2}^{*}\right)\right)\right)$, we have $\mathfrak{S}_{0} \subseteq \mathfrak{M}$. Since $\mathfrak{S}=$ $\mathfrak{S}_{0} \cdot \mathfrak{R}_{\mathfrak{5}}\left(\mathfrak{S}_{2}^{*}\right)$, we have $\mathfrak{S} \subseteq \mathfrak{M}$. We conclude that $\mathfrak{S}_{2}^{*} \in \boldsymbol{U}_{\mathfrak{N}}^{*}(\mathfrak{P} ; 2)$. By 
Lemma 15.43, we have $N\left(\mathfrak{S}_{2}^{*}\right) \subseteq \mathfrak{M}$, and so $\mathfrak{S}_{2}^{*}$ is a $S_{2}$-subgroup of $\mathfrak{S}_{0}$. If $\mathfrak{P} \subseteq \mathfrak{S}^{\prime}$, then $\subseteq=\mathfrak{S}_{2}^{*} \boldsymbol{N}_{\mathscr{\Phi}}(\mathfrak{P}) \subseteq \mathfrak{M}$. Thus, we may assume that $\mathfrak{P} \nsubseteq \mathfrak{S}^{\prime}$.

Since $\mathfrak{S}=\mathfrak{S}_{0} \boldsymbol{N}_{\mathscr{S}}(\mathfrak{P})$, we get $\mathfrak{S}_{0} \nsubseteq \mathfrak{M}$. By minimality of $\mathfrak{S}$, we have $\mathfrak{S}_{0}=\mathfrak{S}_{2}^{*} \mathfrak{Q}$, where $\mathfrak{Q}$ is a $p$-group which admits $\mathfrak{F}$, and $p$ is a prime $\geqq 5$. Of course, $\mathfrak{\Omega}$ is cyclic. Choose $\mathfrak{Y} \in\left\{\boldsymbol{Z}\left(\mathfrak{S}_{2}^{*}\right), \boldsymbol{J}\left(\mathfrak{S}_{2}^{*}\right)\right\}$ with $\mathfrak{Y} \triangleleft \mathfrak{S}_{0}$. Then $N(\mathfrak{Y}) \in \mathscr{S}$, so by maximality of $|\subseteq \cap \mathfrak{M}|_{2}, \mathfrak{S}_{2}^{*}$ is a $S_{2}$-subgroup

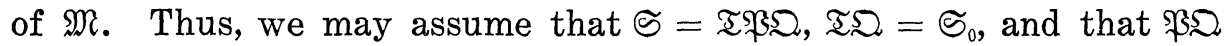
is a Frobenius group. By Lemma 15.42, $\mathfrak{T} \pitchfork \mathfrak{T} \mathfrak{\text { . }}$. Let $\overline{\mathfrak{S}}=\mathfrak{S} / \boldsymbol{O}_{2}(\mathfrak{S})$. Then $\bar{\subseteq}$ is a Frobenius group whose kernel is $\supseteq \boldsymbol{O}_{2}(\mathfrak{S}) / \boldsymbol{O}_{2}(\widetilde{S})$, and $\mathfrak{T P} / \mathrm{O}_{2}(\mathfrak{S})=\mathfrak{G}$, is a cyclic complement of order $3.2^{a}$, with $a \geqq 1$.

Let 3 be a minimal normal subgroup of $\mathfrak{S}$. Since $3 \cap \boldsymbol{Z}(\mathfrak{T}) \neq 1$, and $\Omega_{1}(Z(\mathfrak{I}))^{\sharp} \subseteq \mathscr{F}$, it follows that $\Omega, \mathcal{Z}$ is a Frobenius group. As is well known, this implies that 3 is a free $F_{2}(\mathfrak{V}$-module. Hence, $\mathfrak{P}$ does not centralize $\boldsymbol{Z}(\mathfrak{T})$. This contradiction completes the proof.

LEMma 15.45. Suppose $\mathfrak{P}$ is of prime order $p \geqq 5, \mathfrak{P} \subseteq \mathfrak{M}$, and $C_{\mathfrak{F}}(\mathfrak{P})$ contains an elementary subgroup of ordor 8 . Then $\mathfrak{F} \mathfrak{\beta} \in \mathscr{L}^{*}$.

Proof. We may assume that $\mathfrak{P} \subseteq \mathfrak{D}$. Then $\mathfrak{R} \subseteq \boldsymbol{C}(\mathfrak{P})$, and $\boldsymbol{C}_{\mathfrak{⿰}}(\mathfrak{P})$ admits $\mathfrak{P}$. Hence, $N(\mathfrak{P}) \subseteq \mathfrak{M}$ by Lemma 15.29. Also, of course, since $p \geqq 5$, we have $\mathfrak{P} \subseteq C(\mathfrak{B})$. If $\mathbb{R} \in \boldsymbol{h}_{\mathfrak{M}}^{*}(\mathfrak{P} ; 2)$, then since $\mathfrak{S}_{\mathcal{O}} \mathfrak{D} \triangleleft \mathfrak{M}$, we conclude first that $\left[Z\left(\mathfrak{S}_{0}\right), \mathfrak{D}\right]=1$, and then get that $[Z(\mathbb{Z}), \mathfrak{P}]=1$, since $\boldsymbol{Z}(\mathbb{R}) \subseteq Z\left(\mathfrak{S}_{0}\right)$. This then implies that $N(\mathfrak{R}) \subseteq \mathfrak{M}$, and we can follow the proof of the preceding lemma.

Lemma 15.46. If $|\mathfrak{F}|=4$, then $\mathfrak{D}$ is cyclic, $\mathfrak{M} / \mathfrak{M}^{\prime}$ is a 2-group, and $\mathfrak{F} \mathfrak{F} \in \mathscr{M}^{*}$ for every subgroup $\mathfrak{\beta}$ of $C(\mathfrak{F})$ of odd prime order.

Proof. By Lemma 15.44, we may assume that $|\mathfrak{P}|=p \geqq 5$. Since $\mathcal{F}^{\#} \subseteq \mathscr{F}$, and $\Re(F \subseteq N(\mathfrak{P})$, it follows readily from Lemma 15.29 that $N(\mathfrak{P}) \subseteq \mathfrak{M}$. It is straightforward to verify that $\mathfrak{F} \mathfrak{F} \in \mathscr{A}^{*}$. Since $\mathfrak{D} \subseteq \boldsymbol{C}(\mathbb{S})$, it follows by application of the preceding result to each subgroup of $\mathfrak{D}$ of prime order that $\mathfrak{D} \subseteq \mathfrak{M}^{\prime}$. Since $\mathfrak{D}$ is a $Z$-group, this implies that $\mathfrak{D}^{\prime}=1$. The proof is complete.

LEMMA 15.47. One of the following holds:

(a) $\mathfrak{F}^{\#} \subseteq \mathscr{A}$.

(b) $\boldsymbol{C}(\mathfrak{\mho})$ is a 2-group.

Proof. This is an immediate consequence of Lemmas 15.44 and 15.45 .

LEMMA 15.48. If $\boldsymbol{C}_{\Re}(\mathfrak{F})$ is non cyclic, then $\mathfrak{F}$ is generated by subgroups $\mathfrak{X}$ of order $4|\mathfrak{F}|$ such that $\mathfrak{X}^{\#} \cong \mathscr{F}$, or $|\mathfrak{F}|=8$. 
Proof. Let $\mathfrak{\Omega}$ be an elementary subgroup of $\boldsymbol{C}_{\mathfrak{R}}(\mathfrak{S})$ of order 9 . Let $\widetilde{\mho}_{0}=\boldsymbol{C}_{\widetilde{\mho}}(\mathfrak{D})$, and let $\widetilde{\mho}=\widetilde{\mho}_{0} \times \widetilde{\mho}_{1} \times \cdots \times \widetilde{F}_{r}$, where $\widetilde{\mho}_{i}$ is a fourgroup which admits $\mathfrak{\Omega}$. By Lemma 15.44, $\left(\widetilde{\mathcal{F}}_{0} \widetilde{\mho}_{i}\right)^{\sharp} \subseteq \mathscr{F}, i=1, \cdots, r$, and so we are done.

Lemma 15.49. $\widetilde{F}$ is generated by subgroups $\mathfrak{i}$ of order $4|\mathfrak{F}|$ such that $\mathfrak{Q} ¥ \cong \mathscr{I}$, or $|\mathfrak{F}|=8$.

Proof. We may assume that $|\mathfrak{F}| \geqq 16$. By Lemma 15.48, we may assume that $\mathfrak{Q}=\boldsymbol{C}_{\mathfrak{r}}(\mathfrak{\xi})$ is cyclic. Thus, $|\mathfrak{F}|=4$, and $\mathfrak{F}=\mathfrak{B}$. By Lemma 15.47, we may assume that $C(\mathfrak{F})$ is a 2 -group. Since $\mathfrak{Q}$ is cyclic, we get $\mathfrak{R}=\mathfrak{Q} \times \mathfrak{S}$, where $|\mathfrak{S}|=3$, and $\mathfrak{M} / \mathfrak{M}_{0} \cong \Sigma_{3}$. Let $\mathfrak{I}_{0}=C_{\mathfrak{x}}(\mathfrak{F})$, so that $C(\mathfrak{F})=\mathfrak{I}_{0} \mathfrak{D D}$, and $\mathfrak{D}$ is is a cyclic complement to $\mathfrak{I}_{0}$ in $\boldsymbol{C}(\mathfrak{F})$. Furthermore, since $\mathfrak{M} / \mathfrak{M}_{0} \cong \Sigma_{3}$, we have $\mathfrak{S}_{0} \supseteqq \mathfrak{S}_{0}$, and $\mathfrak{S}_{0} \mathfrak{O D} \triangleleft \mathfrak{M}_{0}$. This implies that $\mathfrak{F} / \mathbb{F}=\overline{\mathscr{F}}$ is a $F_{2} \mathfrak{D} D$-module on which $\mathfrak{D D}$ acts frobeniusly. Since $\mathfrak{D} \mathfrak{D}=\mathfrak{Q} \times \mathfrak{D} \times \mathfrak{S}$, it follows that if $|\mathfrak{D D}|>3$, then for each element $X$ of $\mathfrak{D S}, C_{\tilde{x} / \mathbb{E}}(X)$ is either 1 or of order $\geqq 2^{4}$, and so the lemma follows from Lemma 15.47, Lemma 15.31 (which is applicable since $\mathbb{F}=\mathfrak{B}$ !), together with the well worn observation that $\widetilde{\mho} / \widetilde{S}=\left\langle\boldsymbol{C}_{\widetilde{\mho} / \mathbb{S}}(X) \mid X \in(\mathfrak{S})^{\sharp}\right\rangle$. It remains to treat the case $|\mathfrak{D D}|=3$. In this case, $|\mathfrak{F} / \mathfrak{F}|=2^{2}$ or $2^{4}$. Since $\mathfrak{M}=\mathfrak{S} \Re$, and $\mathfrak{F}, \mathfrak{F} / \mathbb{S}$ are irreducible modules for $\mathfrak{M}$, it follows that $\mathfrak{S}=\boldsymbol{O}_{2}(\mathfrak{M} \bmod \boldsymbol{C}(\mathfrak{F}))$. If $|\widetilde{F} / \mathfrak{F}|=2^{2}$, then $|\widetilde{F}-\mathfrak{F}|=12$, and the orbits of $\Re$ on $\mathfrak{F}-(\mathfrak{F}$ are of sizes 3 and 9 . If $\mathfrak{M}$ is transitive on $\mathfrak{F}-(\mathfrak{F}$, then since $(\mathfrak{F}-(\mathfrak{F}) \cap$ $\mathscr{J} \neq \varnothing$, we get $\mathfrak{F}^{\sharp} \subseteq \mathscr{A}$, and we are done. If $\mathfrak{M}$ is not transitive on $\mathfrak{F}-\mathfrak{F}$, then $\mathfrak{F}$ is 2 -reducible in $\mathfrak{M}$, which is false, since $\mathfrak{B}=\mathfrak{F}$. Suppose $|\mathfrak{F} / \mathfrak{F}|=2^{4}$. Let $\mathfrak{F}=\mathfrak{F} \times \widetilde{F}_{1} \times \widetilde{\mho}_{2}$, where $\widetilde{F}_{i}$ is a four-group admitting $\mathfrak{R}$. Since $\left[\mathfrak{F}_{\mathcal{C}}, \widetilde{F}_{1}\right]$ is a non trivial subgroup of $\mathfrak{F}$ which admits $\Re$, we get $\left[\mathfrak{F}, \widetilde{\mho}_{1}\right]=\mathfrak{F}$. Since $\boldsymbol{C}_{\Re}(\mathfrak{S})=\mathfrak{Q}$ is transitive on $\mathfrak{F}_{1}^{\#}$, it follows that $[\mathfrak{S}, F]=\mathbb{F}$ for all $F \in \mathscr{F}_{1}^{*}$. Since $\mathfrak{F}_{1}^{\sharp} \subseteq \mathscr{F}$, we conclude that $\left(\mathfrak{F}_{\mathfrak{F}}\right)^{*} \cong \mathscr{F}, i=1,2$, and we are home.

We have thus obtained the "correct" analogue of Lemma 13.26(c), for in following $\S 13$, it will be seen that the crucial integer is $|E| \cdot 4$, not 16 .

We come to the analogue of Lemma 13.29. This lemma becomes almost unmanageable. In order to handle the difficulties, we introduce some notation. For each integer $f$, let $\mathfrak{V}_{f}=\mathfrak{V}_{f}(\mathfrak{M})=\{I \mid I$ is an involution of $\mathfrak{M}$ and $\left.|[\mathfrak{F}, I]|=2^{f}\right\}$. The set $\mathfrak{U}_{1}$ causes no trouble.

Lemma 15.50. If $I \in \mathfrak{Q}_{1}$, then $[\widetilde{F}, I]=\langle J\rangle$, and $J \in \mathscr{F}_{\text {. }}$

Proof. If $J \in \mathfrak{F}$, we are done. Suppose $I \in(\mathfrak{5}$. By Lemma 15.47, we may assume that $C(\mathfrak{F})$ is a 2-group. In this case, $\mathfrak{D}_{0}=$ $\boldsymbol{O}_{2}\left(\mathfrak{M}_{0} \bmod \boldsymbol{C}(F)\right) \subseteq \mathfrak{S}_{2}$, and $\left|\mathfrak{S}_{2} / \mathfrak{D}_{0}\right| \leqq 2$, as $\mathfrak{S}_{2} / \mathscr{D}_{0}$ acts faithfully on $\mathbb{E}$. 
If $\mathfrak{D}_{1} / \mathfrak{D}_{0}$ is an odd order subgroup of $\mathfrak{M}_{0} / \mathfrak{D}_{0}$ which admits $I$ and is of order $>3$, then $\boldsymbol{C}_{\Phi_{1}}(J) \nsubseteq \mathfrak{D}_{0}$, and so $J \in \mathscr{F}$, by Lemma 15.44 and 15.45 . If no such $\mathfrak{D}_{1}$ exists, then we get $\mathfrak{M}=\mathfrak{S} \mathfrak{R},|\mathfrak{R}|=3^{2}$. $\mathfrak{M} / \mathfrak{M}_{0} \cong \sum_{3}$. Hence, $|\mathfrak{F}|=2^{4},|\mathfrak{F}|=4$, so $\mathfrak{F}^{\sharp} \subseteq \mathscr{F}$, by Lemma 15.49. The proof is complete.

The critical sets are $\mathfrak{U}_{2}$ and $\mathfrak{U}_{3}$. These are vital. To focus our attention on the difficulties of Lemma 13.29, let

$$
\begin{aligned}
\mathfrak{B}_{2}= & \left\{I \in \mathfrak{A}_{2}, I \in C(\mathfrak{F}),[\mathfrak{F}, I]^{\sharp} \nsubseteq \mathscr{F}\right\}, \\
\mathfrak{B}_{3}= & \left\{I \in \mathfrak{U}_{3}, I \in C(\mathfrak{F}), \text { there is a hyperplane } \mathfrak{B}_{0} \text { of } \mathfrak{B}\right. \\
& \text { such that } \left.\mathfrak{B}_{0} \supseteqq \boldsymbol{C}_{\mathfrak{F}}(I), \text { and }\left[\mathfrak{B}_{0}, I\right]^{\sharp} \cap \mathscr{J}=\varnothing \cdot\right\}, \\
& \mathfrak{B}=\mathfrak{B}_{2} \cup \mathfrak{B}_{3} .
\end{aligned}
$$

Lemma 15.51. If $\mathfrak{B} \neq \varnothing$, then the following hold:

(a) $\mathfrak{D}=1$.

(b) $|\Re| \leqq 3^{3}$ and $\mathfrak{R}$ is of exponent 3 .

Proof. Choose $I \in \mathfrak{B} \cap \mathfrak{T}$. Since $\mathfrak{B}$ is a union of conjugacy classes of $\mathfrak{M}$, and $\mathfrak{B} \neq \varnothing, I$ exists. Since $\mathfrak{B} \neq \varnothing$, Lemma 15.47 implies that $\boldsymbol{C}(\widetilde{\mho})$ is a 2-group.

Suppose $\mathfrak{D} \neq 1$. Let $\mathfrak{D}^{*}=\boldsymbol{F}(\mathfrak{D})$. Then $\mathfrak{D}^{*}$ centralizes $\boldsymbol{F}(\mathfrak{D} \Re)$, and so $\mathfrak{S}_{0} \mathfrak{D}^{*} \triangleleft \mathfrak{M}$. Hence, $\mathfrak{S}_{0} \mathfrak{D}_{1}^{*} \triangleleft \mathfrak{M}$ for every subgroup $\mathfrak{D}_{1}^{*}$ of $\mathfrak{D}^{*}$, and so $\widetilde{F}=\left[\mathfrak{F} \times\left[\mathfrak{F}, \mathfrak{D}_{1}^{*}\right]\right.$ for all non identity subgroups $\mathfrak{D}_{1}^{*}$ of $\mathfrak{D}^{*}$.

First, suppose that $I$ does not centralize $\mathfrak{S}_{0} \mathfrak{D}^{*} / \mathscr{S}_{0}$. We can then choose $\mathfrak{D}_{1}^{*}$ of prime order in $\mathfrak{D}^{*}$ such that $I$ inverts $\mathfrak{S}_{0} \mathfrak{D}^{*} / \mathfrak{S}_{\mathcal{C}}$. In this case, it follows that $\mid \mathfrak{F} /\left(\mathfrak{F} \mid=2^{2 k}\right.$, and that $C_{\mathscr{\mho} / \mathbb{E}}(I)=2^{k}$. Hence, $\mathrm{k} \leqq 3$, as $I \in \mathfrak{B}$, and so one of the following holds:

( $\alpha) \quad\left|\mathfrak{D}_{1}^{*}\right|=5$ and $|\mathfrak{F} / \mathfrak{F}|=2^{4}$,

( $\beta$ ) $\left|\mathscr{D}_{1}^{*}\right|=7$ and $\mid \mathfrak{F} /\left[\mathfrak{G} \mid=2^{6}\right.$.

If $(\alpha)$ holds, then as $\Re D$ acts faithfully on $\widetilde{F}$, we get $|\Re|=3^{2},|\mathfrak{D}|=5$. If $(\beta)$ holds, we get $|\Re|=3^{2},|\mathfrak{D}|=7$.

Suppose $(\alpha)$ holds; then $\mathfrak{R}^{0}=\boldsymbol{C}_{\mathfrak{r}}(\mathfrak{F} / \mathfrak{F})$ is of order 3 , and $\mathfrak{F}=$ $\boldsymbol{C}_{\widetilde{F}}\left(\Re^{0}\right) \times \mathfrak{F}, \mathfrak{F}=\left[\mathfrak{F}, \mathfrak{R}^{0}\right]$. Since $\widetilde{F}$ is not 2-reducible in $\mathfrak{M}$, we get that $\mathfrak{D}_{0}=\boldsymbol{O}_{2}\left(\mathfrak{M}_{0} \bmod \boldsymbol{C}(\mathfrak{F})\right) \supset \boldsymbol{C}(\mathfrak{F})$, and so $\left[\mathfrak{D}_{0}, \mathfrak{F}\right]=\mathfrak{F}$. If $F \in \boldsymbol{C}_{\mathfrak{F}}\left(\mathfrak{R}^{0}\right)^{\sharp}$, then

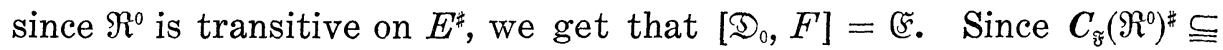
$\mathscr{F}$, we get that $\mathfrak{F}^{*} \subseteq \mathscr{F}$, against $\mathfrak{B} \neq \varnothing$.

Suppose $(\beta)$ holds; again let $\mathfrak{R}^{0}=\boldsymbol{C}_{\mathfrak{R}}(\mathfrak{\mho} / \mathfrak{\Im})$. Choose $F \in \boldsymbol{C}_{\widetilde{\mho}}\left(\mathfrak{R}^{0}\right)^{\sharp}$. Then $\left[\mathfrak{D}_{0}, F\right]$ admits $\Re^{0}$ and is a non identity subgroup of $\mathfrak{F}$, so that

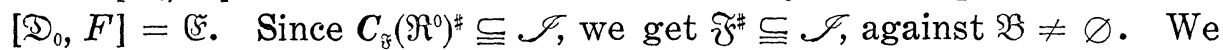
conclude that $I$ centralizes $\mathscr{S}_{0} \mathfrak{D}^{*} / \mathfrak{S}_{2}$.

Since $I$ centralizes $\mathscr{S}_{0} \mathfrak{D}^{*} / \mathfrak{S}_{0}$, it follows that $\mathfrak{D}^{*}$ normalizes $[\mathfrak{F}, I] \mathfrak{F}$. Since $[\widetilde{F}, I]^{*} \nsubseteq \mathscr{F}$, we have $[\widetilde{\mho}, I] \nsubseteq \mathfrak{r}$. Since no non identity element of $\mathfrak{D}^{*}$ has a non trivial fixed point on $\widetilde{F} / \mathfrak{F}$, the only possibility is 
that $|[\mathfrak{F}, I]|=8,[\widetilde{F}, I] \cap \mathfrak{F}=1,\left|\mathfrak{D}^{*}\right|=7$. Since $\mathfrak{D}^{*}=\boldsymbol{F}(\mathfrak{D})$, we get $\mathfrak{D}^{*}=\mathfrak{D}$.

Choose $\mathfrak{P}=\langle P\rangle$ of order 3 in $C_{\mathfrak{m}}(\mathfrak{D})$ with $P^{I}=P^{-1}$; $\mathfrak{P}$ exists since $\Re \mathfrak{N D} \subseteq C(\mathfrak{D})$, and since $I \nsubseteq O_{2}(\mathfrak{M})$. Let $\mathfrak{F}^{*}=[\mathfrak{F}, \mathfrak{\Re}]$. Since $I \in \boldsymbol{C}(\mathfrak{F})$, so also $\mathfrak{P} \leqq \boldsymbol{C}(\mathfrak{F})$. Thus, $\left|\mathfrak{F}^{*}\right|=2^{2 f *}$, where $f^{*} \leqq 3$. Since $\mathfrak{F}^{*}$ admits $\mathfrak{D}$, we have $f^{*}=3$. Let $\mathfrak{Q} \subseteq C_{\mathfrak{n}}(\mathfrak{P}),|\mathfrak{Q}|=9$, $\mathfrak{\text { elemen- }}$ tary. Then $\mathfrak{D}$ acts faithfully on $\mathfrak{F}^{*}$, since $\mathfrak{F}^{*} \supset[\mathfrak{F}, I]$. Hence, we get that $G L(6,2)$ has an abelian subgroup of type $(3,3,7)$. This is false, and so (a) holds, $\mathfrak{D}=1$.

Now for (b). By Lemma 15.36, $I$ normalizes ${\mathfrak{\mathcal { F } _ { 0 }}}^{\Re^{T}}$ for some $T \in \mathfrak{T}$. We may thus choose $I \in \mathfrak{B} \cap \mathfrak{I} \cap N\left(\mathfrak{S}_{0} \Re\right)$.

Let $\Re^{1}=C_{\Re}(\mathfrak{F}), \mathscr{S}_{\mathfrak{C}}^{0}=\boldsymbol{C}_{\mathfrak{F}_{0}}(\mathfrak{S})$. First, suppose that $\Re^{1}$ is cyclic. In this case, $\mathfrak{R}=\mathfrak{R}^{1} \times \mathfrak{Q},|\mathfrak{Q}|=3$, since $\mathfrak{R}$ is metacyclic, whence abelian. Since $I \in \mathfrak{M}_{0}$, and $I \notin \mathfrak{S}_{0}$, we get $I \notin \boldsymbol{O}_{2}\left(\mathfrak{M}_{0}\right)$, and so $I$ inverts an element of $\mathfrak{M}_{0}$ of order $\left|\mathfrak{R}^{1}\right|$. We assume without loss of generality that $I$ inverts $\mathfrak{R}^{1}$. Since $I \in \mathfrak{B}$, and since $\mathfrak{F}=\widetilde{F} \times\left[\mathfrak{F}, \Re^{1}\right]$, it follows that $\left|\mathfrak{R}^{1}\right| \leqq 3^{2}$. Furthermore, if $\left|\Re^{1}\right|=3^{2}$, then $I \in \mathfrak{N}_{3}$ and $[\mathfrak{F}, J] \subset\left[\mathfrak{F}, \mathfrak{R}^{1}\right]$. Let $\widetilde{\Re}$ be a $S_{3}$-subgroup of $\boldsymbol{C}_{\mathfrak{n}}\left(\Re^{1}\right)$, and let $\widetilde{\mathfrak{Q}}=\boldsymbol{C}_{\mathfrak{\Re}}\left(\left[\widetilde{\mho}, \Re^{1}\right]\right)$. Thus, $|\widetilde{\mathfrak{D}}|=3$, and so $\left[\mathfrak{F}, \mathfrak{R}^{1}\right] \subseteq \mathscr{F}$, against $I \in \mathfrak{B}$. So $\left|\mathfrak{R}^{1}\right|=3,|\mathfrak{R}|=3^{2}$, and we are done.

We may now assume that $\Re^{1}$ is non cyclic. In this case, $\Re^{1} \supseteqq \Omega_{1}(Z(\Re))$, since $3 \in \pi_{2}$; and since $\Re^{1}$ is faithful on $\mathfrak{F} / \mathfrak{F}$, so is $\Re$. Let $C_{\mathfrak{1}} 1_{\mathfrak{p}_{0} / \mathfrak{S}_{0}}(I)=\Re_{2} \mathfrak{S}_{0} / \mathfrak{S}_{0}$, with $\Re_{2} \subseteq \Re^{1}$. Thus, $\Re_{2}$ acts faithfully on

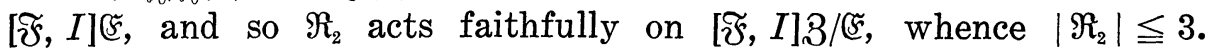
Suppose $\Re_{2}=1$. In this case, $I$ inverts $\mathscr{S}_{0} \Re^{1} / \mathscr{L}_{0}$, and so $\mathfrak{S}_{0} \Re^{1} \triangleleft \mathfrak{M}$, whence $\mathfrak{F}=\mathfrak{F} \times\left[\mathfrak{F}, \Re^{1}\right]$. Then $\left|\left[\widetilde{\mho}, \Re^{1}\right]\right|=2^{2 f *}$, where $f^{*} \leqq 3$. Since $\Re$ acts faithfully on $\mathfrak{\mho} / \mathfrak{F}$, and since $3 \in \pi_{2}, \Re$ is isomorphic to a subgroup of $G L(6,2)$ which has no elementary subgroup of order $3^{3}$. Hence, $|\Re| \leqq 3^{3}$, and since $\Re$ is not a metacyclic group of order $3^{3}$, we get that $\Re$ has exponent 3 . It remains to treat the case $\left|\Re_{2}\right|=3$.

Since $\left\langle\mathfrak{S}_{0}, \mathfrak{R}^{1}, I\right\rangle / \mathfrak{F}_{0}$ is supersolvable, there is $\mathfrak{U} \in \mathscr{S} \mathscr{C} \mathscr{N}\left(\mathfrak{R}^{1}\right)$ with $\mathfrak{2} \mathcal{S}_{0} \triangleleft \mathfrak{F}_{0} \Re^{1}\langle I\rangle$. Suppose $|\mathfrak{U}| \geqq 27$. In this case, $I$ inverts an element $A$ of $\mathfrak{Q} \mathfrak{S}_{0}$ of order 9 , and we may assume that $A \in \mathfrak{N}$. Let $\tilde{\mathfrak{A}}=\langle A\rangle$. Then $|[\mathfrak{F}, \tilde{\mathfrak{N}}]|=2^{6}$, and $[\mathfrak{F}, \tilde{\mathfrak{N}}] \supset[\mathfrak{F}, I], I \in \mathfrak{A}_{3}$. Since $|\mathfrak{X}| \geqq 27$, it follows that $\mathfrak{A} \cap C([\widetilde{\mathfrak{F}}, \tilde{\mathfrak{X}}]) \neq 1$, so $[\widetilde{\mathfrak{F}}, \tilde{\mathfrak{A}}]^{*} \cong \mathscr{F}$, which is false. So $|\tilde{\mathfrak{A}}| \leqq 9$. If $|\tilde{\mathfrak{A}}|=3$, then $|\mathfrak{R}|=9$, and we are done. Suppose $|\mathfrak{A}|=9$. If $\mathfrak{A}=\mathfrak{R}^{1}$, then $|\mathfrak{R}| \leqq 3^{3}$. If $\Re$ has exponent 3 , we are done, so suppose $\Re$ is abelian of exponent 9 (and order 27). Since $\left|\Re_{2}\right|=3$, and since $I \in C(\mathfrak{s})$, and since $\Re^{1}$ is non cyclic, it follows that $\Re=\mathfrak{\Omega} \times \mathfrak{S},|\mathfrak{Q}|=$ $3, \mathfrak{Q}$ is inverted by $I$, and $I$ does not invert $\mathfrak{R}^{1} \mathfrak{E}_{0} / \mathfrak{F}_{0}$. Thus, $I$ normalizes $\Re \mathscr{S}_{0} / \mathscr{S}_{0}$, and $C_{\mathfrak{R} \mathfrak{F}_{0} / \mathfrak{F}_{0}}(I)$ is cyclic of order 9 . But then if $X$ is an element of $\Re$ of order 9 such that $\boldsymbol{C}_{\Re \mathfrak{q}_{0} / \mathfrak{F}_{0}}(I)=\left\langle X \mathfrak{S}_{0}\right\rangle$, then $X^{3}$ centralizes [₹्צ, $\left.J\right] \mathfrak{F}$, so that $[\widetilde{\digamma}, J]^{\sharp} \subseteq \mathscr{F}$. This is false, so this case does not arise. 
It only remains to treat the case $|\mathfrak{A}|=9, \mathfrak{A} \subset \mathfrak{R}^{1}$. In this case, we get $\left|\Re^{1}\right|=3^{3}$, so that if $\Re^{1}=\Re$, we are done, since $\Re$ is not a metacyclic group of order $3^{3}$. Suppose then that $\mathfrak{A} \subset \Re^{1} \subset \Re,|\Re|=3^{4}$. Let $\mathbb{B}=\left[\Re^{1}, \Re^{1}\right]$. If $I$ inverts $\mathfrak{S}_{0} \mathbb{B} / \mathscr{\mathcal { S }}_{0}$, then since $\left.\left.\mathfrak{F}=\mathbb{F} \times\right] \mathfrak{F}, 3\right]$, it follows that $|\mathfrak{F} / \mathfrak{F}|=2^{6}$, whence $\Re$ is isomorphic to a $S_{3}$-subgroup of $G L(6,2)$. This is false, since $2 \in \pi_{2}$. Thus, $I$ centralizes $\mathfrak{S}_{0} \mathfrak{3} / \mathfrak{H}_{0}$, and so $3=\Re_{2}$. However, in this case, $\mathfrak{F} / \mathfrak{F}$, as module for $\Re^{1}$, is the direct sum of $r(\geqq 1)$ modules, each of cardinal $2^{6}$. If $r>1$, it is easy to see that $|[\mathfrak{F} / \mathfrak{F}, I]| \geqq 2^{4}$, against $I \in \mathfrak{B}$. Hence, $r=1,|\mathfrak{F} / \mathfrak{F}|=2^{6}$, and so the same argument shows that $\Re$ has an elementary subgroup of order 27. The proof is complete.

We next record a partial analogue of Lemma 13.31.

LEMMA 15.52. One of the following holds:

(a) $\mathfrak{F}^{\prime} \subseteq \mathscr{I}$.

(b) $\boldsymbol{C}\left(\mathfrak{F}_{0}\right) \subseteq \mathfrak{M}$ for every subgroup $\mathfrak{F}_{0}$ of index $4|\mathfrak{F}|$ in $\mathfrak{F}$.

(c) $\mathfrak{D}=1,|\Re| \leqq 3^{3}$, and $\mathfrak{R}$ has exponent 3 .

Proof. We may assume that $\mathfrak{F}^{\sharp} \nsubseteq \mathscr{F}$. Thus, $\boldsymbol{C}(\mathfrak{\mho})$ is a 2-group, by Lemma 15.47. If $|\mathfrak{F}|=4$ and $|\mathfrak{F}|=16$, then $\mathfrak{F}^{\sharp} \subseteq \mathscr{F}$, by Lemma 15.49. So we may assume that either $|\mathfrak{F}|=2$ or $|\mathfrak{F}| \neq 16$. Since $C(\mathfrak{F})$ is a 2 -group, we have $|\mathfrak{F}| \geqq 16$, and if $|\mathfrak{F}|=16$, then $|\mathfrak{F}|=4$ is forced. So we may assume that $|\mathfrak{F}| \geqq 32$.

If $\mathfrak{D} \neq 1$, it follows that $\left|C_{\S / \mathbb{E}}(X)\right| \geqq 2^{3}$ for some element $X$ of $\Re$ of order 3. Then $\boldsymbol{C}_{\mathfrak{F}}(X)^{\sharp} \subseteq \mathscr{J}$, so if $\boldsymbol{C}_{\Re}(X) \cap \mathfrak{\mho}_{0} \neq 1$, (b) holds. We may assume that $\boldsymbol{C}_{\mathfrak{\xi}}(X) \cap \mathfrak{\mho}_{0}=1$. Thus, $X$ does not centralize (r. Thus, $|\mathfrak{D}|=5$ or 7 and $C_{\mathfrak{r}}(\mathbb{F})$ is cyclic, whence $\mathfrak{R}^{\prime}=1$. Since $C_{\mathfrak{r}}(\mathfrak{F})$ is cyclic, we get $\mathfrak{F} \times C_{\Re}(X) \triangleleft \mathfrak{M}$, so $|\mathfrak{F}|=2^{6}$ or $2^{5}$, whence $\left|\mathfrak{F}_{0}\right|=4$

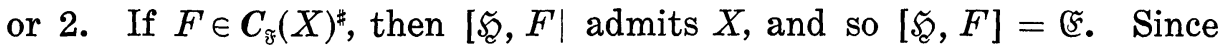
$C_{\Im}(X)^{\sharp} \subseteq \mathscr{J}$, we get $\mathfrak{F}^{\sharp} \subseteq \mathscr{F}$.

We may now assume that $\mathfrak{D}=1$.

Now $\left|\mathfrak{F}_{:} \mathfrak{F}_{0}\right|=4|\mathfrak{F}|$, and so if $X$ is any element of $\mathfrak{M}$ of order 3 , then since we may assume that $\widetilde{\mho}_{0} \cap \mathscr{J}=\varnothing$, we get $\boldsymbol{C}_{\widetilde{\mho}_{0}}(X)=1$, whence $\left|\boldsymbol{C}_{\mathfrak{J}}(X)\right| \leqq 4|\mathfrak{F}|$. Thus, $\Re$ has no non cyclic abelian subgroup of order 27, and so (c) holds.

LEMMA 15.53. $\mathfrak{D}=1, \Re$ is of exponent $3,|\Re| \leqq 3^{3}$, and one of the following holds:

(a) $\mathfrak{B} \neq \varnothing$.

(b) $\boldsymbol{C}\left(\mathfrak{\mho}_{0}\right) \nsubseteq \mathfrak{M}$ for some non identity subgroup $\mathfrak{\mho}_{0}$ of $\mathfrak{F}$ of index at most $4 \mid$ \& $\mid$.

Proof. If neither (a) nor (b) holds, we may follow the argument of $\S 13$ to a contradiction. So we get that either (a) or (b) holds. 
In either case, this lemma follows from Lemma 15.51, or 15.52, according as (a) or (b) holds.

LEMMA 15.54. If $\mathfrak{l}$ is a four-group of $\mathfrak{M}$ and $\mathfrak{U} \cap C(\mathfrak{F})=1$, then $[\mathfrak{F}, \mathfrak{u}] \cap \mathscr{F} \neq \varnothing$.

Proof. If $\mathfrak{u} \nsubseteq C(\mathfrak{E})$, the lemma is trivial, since $\mathfrak{F} \equiv \mathscr{F}$. So suppose $\mathfrak{H} \subseteq C(\mathfrak{5})$. By Lemma 15.36, we may assume that $\mathfrak{H}$ normalizes $\mathfrak{S} \Re$. If $\mathfrak{U}_{0}=\mathfrak{U} \cap \mathfrak{F} \neq 1$, then $1 \subset\left[\mathfrak{U}_{0}, \mathfrak{F}\right] \subseteq \mathfrak{F}$, and we are done. We may assume that $\mathfrak{B} \cap \mathfrak{U}=1$. We argue that $\mathfrak{U}$ has an involution $U$ such that for some subgroup $\widetilde{\mathfrak{R}}$ of order 9 of $\Re, U$ inverts $\mathfrak{S} \widetilde{\mathfrak{R}} / \mathscr{S}$. This is obvious, by Lemma 5.31. Thus, by 2 applications of Lemma 5.36, we get that $U$ inverts a $S_{3}$-subgroup $\Re^{*}$ of $\mathfrak{S} \Re$. Let $\mathfrak{F}^{*}=\left[\mathfrak{F}, \Re^{*}\right]$. By Lemma 15.53, $\mathfrak{F}^{*} \nsubseteq \mathscr{F}$, and so $\Re^{*}$ acts faithfully on $\mathfrak{F}$, by Lemma 15.47. Let $\mathfrak{F}^{* *}$ be a minimal normal subgroup of $\mathfrak{\mho}^{*} \Re *\langle U\rangle$. Then $\mathfrak{F}^{* *}$ is a four-group and $\mathfrak{F}^{* * *} \subseteq \mathscr{F}$. Since $1 \subset\left[\mathfrak{F}^{* *}, U\right] \subset \mathfrak{F}^{* *}$, the proof is complete.

LEMma 15.55. $\mathfrak{M}$ does not contain a four-group $\mathfrak{U}$ such that $\mathfrak{U}$ acts faithfully on $\mathfrak{F} / \mathfrak{F}$ and $C_{\mathfrak{F}}(\mathfrak{H})$ is a hyperplane of $F$.

Proof. Since $\mathfrak{F} / \mathfrak{G}$ is a chief factor of $\mathfrak{M}$, this lemma follows from Lemma 5.31 .

Lemma 15.56. One of the following holds:

(a) $\mathfrak{T} \in \mathscr{L}^{*}$.

( b ) $N(\mathfrak{S}) \nsubseteq \mathfrak{M}$, where $\mathfrak{W}=\mathbb{V}\left(\operatorname{ccl}_{\mathfrak{S}}(\mathfrak{F}) ; \mathfrak{I}\right)$.

Proof. Suppose $\mathfrak{I} \notin \mathscr{C}^{*}$. Choose $\subseteq$ to be a solvable subgroup of (S) which contains $\mathfrak{I}$ and is minimal subject to $\mathfrak{S} \nsubseteq \mathbb{M}$. If $\mathfrak{M} \triangleleft \mathfrak{S}$, we are done. Suppose $\mathfrak{M} \pitchfork \subseteq$. By minimality of $\mathfrak{S}$, we have $\subseteq=\mathfrak{T} \mathfrak{F}$, where $\mathfrak{P}$ is a cyclic $p$-group for some odd prime $p$. Let $\mathfrak{P}=\langle P\rangle$, $\mathfrak{S}_{0}=\boldsymbol{O}_{2}(\mathfrak{S}), \mathfrak{S}_{1}=\left[\mathfrak{S}_{0}, \mathfrak{P}\right], \mathfrak{S}_{2}=\boldsymbol{C}_{\widetilde{\Im}_{0}}(\mathfrak{P})$. Choose $G$ in $\mathbb{S}$ such that $\mathfrak{F}^{G} \subseteq \mathfrak{S}$, $\mathfrak{F}^{G} \nsubseteq \mathfrak{S}_{0}$. Since $\mathfrak{B} \sharp \mathfrak{S}, G$ exists.

Let $\mathfrak{i}$ be a minimal normal subgroup of $\widetilde{\varsigma}$, and let $\widetilde{\mho}_{1}=\mathfrak{F}^{G} \cap \mathfrak{S}_{0}$. Then $\widetilde{F}_{1}$ is a hyperplane of $\widetilde{F}^{G}$, and so $\mathfrak{u} \subseteq C\left(\widetilde{\mho}_{1}\right) \subseteq \mathfrak{M}^{G}$. Hence, if $\widetilde{F}^{G}=\widetilde{F}_{1} \times\langle F\rangle$, then $[\mathfrak{M}, F]^{*} \subseteq \mathscr{J}^{G}$. Since $[\mathfrak{M}, F] \cap \boldsymbol{Z}(\mathfrak{T}) \neq 1$, it follows that $\mathfrak{T} \subseteq \mathfrak{M}^{G}$. Since $N(\mathfrak{I}) \subseteq \mathfrak{M}$, it follows that $\mathfrak{M}^{G}=\mathfrak{M}$. Hence, $\widetilde{\mho}^{G}=\mathfrak{F}$. We may assume that $F$ inverts $\mathfrak{P}$.

Let $\widetilde{\mho}_{2}=\left[\mathfrak{S}_{1}, F\right]$. Then $\widetilde{S}_{1}=\left\langle\widetilde{\mho}_{2}, \mathfrak{F}_{2}^{P}\right\rangle$, and since $\widetilde{\mho}_{2} \triangleleft \mathfrak{S}_{1}$, it follows that $\mathfrak{S}_{1}^{\prime} \subseteq \widetilde{\mho}$. Thus, $\mathfrak{P}$ centralizes $\mathfrak{S}_{1}^{\prime}$, since $F$ does, and since $\mathfrak{P}$ normalizes $\mathfrak{S}_{1}^{\prime}$. Since $\mathfrak{S}_{1}^{\prime} \triangleleft \mathfrak{I}$, and since $\Omega_{1}(Z(\mathfrak{Z}))^{*} \subseteq \mathscr{F}$, while $\mathfrak{P} \nsubseteq \mathfrak{M}$, it follows that $\mathfrak{S}_{1}$ is abelian, and so $\mathfrak{S}_{1}=\mathfrak{F}_{2} \times \mathfrak{F}_{2}^{P}$. Hence, $\mathfrak{S}_{0}=\mathfrak{S}_{1} \mathfrak{S}_{2}$, 
and $\mathfrak{S}_{1} \cap \mathfrak{S}_{2}=1$. Hence, $\boldsymbol{C}_{\widetilde{\Xi}_{2}}\left(\mathfrak{S}_{1}\right) \triangleleft \mathfrak{T}$, and so $\boldsymbol{C}_{{\Im_{2}}_{2}}\left(\mathfrak{S}_{1}\right)=1$. Suppose $X \in \mathfrak{F}_{1}$. Then $X=X_{1} X_{2}, X_{i} \in \mathfrak{S}_{i}$. Since $\mathfrak{F}^{\prime}=1$, we get that $X_{2}$ centralizes $\mathfrak{F}_{2}$, so $X_{2}$ centralizes $\mathfrak{\Im}_{2}^{P}$, whence $X_{2} \in C_{\widetilde{\Im}_{2}}\left(\mathfrak{S}_{1}\right)$, so that $X_{2}=1$. Hence, $\mathfrak{F}_{=}=\mathfrak{F}_{2} \times\langle F\rangle, \mathfrak{\mho}_{2}=\mathfrak{F}_{1}$. By Lemma 15.55, $\mathfrak{F}_{2}^{P} \cap \mathfrak{S}_{\mathrm{C}}$ is of index at most 2 in $\mathfrak{F}_{2}^{P}$. Since $\mathfrak{F}_{2}^{P} \cap \mathfrak{S}_{\mathcal{C}}$ stabilizes the chain $\mathfrak{F} \supset \mathfrak{F} \supset 1$, and centralizes the hyperplane $\mathfrak{\mho}_{1}$ of $\widetilde{\mho}$, it follows that $\mid \mathfrak{\mho}_{2}^{P} \cap \mathfrak{S}_{2}: \mathfrak{\mho}_{2}^{P} \cap$ $\mathfrak{F} \cap C(F) \mid \leqq 4$. But $\mathfrak{F}_{2}^{P}$ acts faithfully on $\mathfrak{F}$, and so we get $\mathfrak{F}_{2}=\mathfrak{F}_{1}$ of order at most 8 , so that $|\mathfrak{F}| \leqq 16$. This is false, since $\mathfrak{F}^{\sharp} \nsubseteq \mathscr{F}$. The proof is complete.

LEMmA 15.57. If $\mathfrak{X}$ is a subgroup of $\mathfrak{R}$ of order 3 , and $\mathbb{R} \in \boldsymbol{Y}_{\mathfrak{R}}^{*}(\mathfrak{U} ; 2)$, then the following hold:

(a) $C_{\mathfrak{\&}}(\mathfrak{U}) \neq 1$.

(b) $N(\mathfrak{R}) \subseteq \mathfrak{M}$.

Proof. Let $3=\Omega_{1}(Z(\mathscr{E}))$. We argue that $|3| \leqq 4$. If $|3| \geqq 8$, then 3 contains an element of $\mathscr{F}$. Since $\mathscr{F}^{*}=\varnothing$, it follows that $\Re$ acts faithfully on 3 . But then 3 is 2 -reducible in $\mathfrak{M}$, against $|\mathfrak{B}| \leqq 4$. Hence, $|\mathfrak{Z}| \leqq 4$. Thus, if $\mathfrak{S} \subseteq \mathbb{R} \subseteq \mathfrak{T}$, then $\boldsymbol{Z}(\mathfrak{Q}) \subseteq Z(\mathfrak{F})$, and so $\left|\Omega_{1}(Z(\Omega))\right| \leqq 4$. If $\Re$ does not centralize $Z(\mathscr{S})$, we get $\Omega_{1}(Z(\mathscr{S}))^{\#}=$ $\mathfrak{B}^{\sharp} \subseteq \mathscr{I}$. If $\Re$ does centralize $Z(\mathscr{K})$, we also get $\Omega_{1}(\mathscr{Z}(\mathfrak{K}))^{\sharp} \subseteq \mathscr{F}$. Hence, $\Omega_{1}(Z(\mathfrak{S}))^{\sharp} \subseteq \mathscr{F}$, and so (b) follows.

Suppose $\boldsymbol{C}_{\mathfrak{R}}(\mathfrak{U})=1$. Since every non central element of $\Re$ has fixed points on $\mathfrak{F}$, it follows that $\mathfrak{A} \subseteq Z(\Re)$. Since $\Re^{\prime}$ centralizes $\mathfrak{F}$, it follows that $\mathfrak{R}^{\prime}=1$. Thus, $|\mathfrak{R}|=3^{2}$, and $\mathfrak{S} \Re \triangleleft \mathfrak{M}$. Thus, [R, $\left.\mathfrak{N}\right] \subseteq$ $\mathfrak{L} \cap \mathfrak{S} \mathfrak{R} \subseteq \mathfrak{S}$, and so $\mathfrak{Z}=\mathfrak{F}$. Since $\boldsymbol{C}_{\mathfrak{R}}(\mathfrak{F})=\mathfrak{B}$ is of order 3 , we have $\mathfrak{S} \mathfrak{B} \triangleleft \mathfrak{M}$. Suppose $\mathfrak{H} \mathfrak{A} \triangleleft \mathfrak{M}$. In this case, since $\mathfrak{L}=\mathfrak{K}$, we get $|\mathfrak{T}: \mathfrak{S}|=2$, and $\mathfrak{I}=\langle\mathfrak{S}, T\rangle$ where $T$ inverts $\mathfrak{R}$. Since $\mathfrak{F} / \mathfrak{F}$ is a chief factor of $\mathfrak{M}$, we conclude that $|\mathfrak{F} / \mathfrak{F}|=4,|\mathfrak{F}|=16$. This is false,

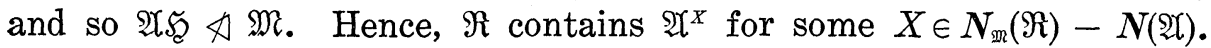
Thus, $\Re$ has precisely 2 subgroups $\mathfrak{N}_{1}, \mathfrak{A}_{2}$ of order 3 which have non trivial fixed points on $\mathfrak{S}$, and so by Lemma 5.58, $\mathfrak{F}=\boldsymbol{C}_{\mathfrak{5}}\left(\mathfrak{U}_{1}\right) \times \boldsymbol{C}_{\mathfrak{5}}\left(\mathfrak{U}_{2}\right)$. This violates $|\mathfrak{B}| \leqq 4$. The proof is complete.

LEMma 15.58. $N(\mathfrak{W}) \varsubsetneqq M$, where $\mathfrak{W}=V\left(\operatorname{ccl}_{\circledast}(\mathfrak{\mho}) ; \mathfrak{I}\right)$.

Proof. Suppose false. By Lemma 15.56, we have $\mathfrak{T} \in \mathscr{L}^{*}$. Since $\mathfrak{F}^{\sharp} \leqq \mathscr{J}$, we have $\mathscr{S} \neq \varnothing$, where

$$
\mathscr{S}=\{\mathfrak{S} \mid \mathfrak{F} \subseteq \mathfrak{S} \nsubseteq \mathbb{M}, \quad \mathfrak{S} \text { is solvable, } \mathfrak{S} \cong \mathbb{S}\} \text {. }
$$

Choose $\mathfrak{S} \in \mathscr{S}$ with $|\mathfrak{S} \cap \mathfrak{M}|_{2}$ maximal, and with this restriction, minimize $\mathfrak{S}$. Thus, $\mathfrak{S}=\mathfrak{S}_{2} \Re$, where $\mathfrak{S}_{2}$ is a $S_{2}$-subgroup of $\mathfrak{S}$ and of $\subseteq \cap \mathfrak{M}$, and $\mathfrak{P}$ is a cyclic $p$-group for some odd prime, and $\boldsymbol{O}_{2}^{\prime}(\mathfrak{S})=1$. Also, if $\widetilde{S}_{2} \subset \widetilde{\mathfrak{S}}_{2}$, where $\widetilde{\Im}_{2}$ is a 2-subgroup of $\mathbb{S}$, then $\widetilde{\mathfrak{S}}_{2} \subseteq \mathfrak{M}$, and 
$\widetilde{\mathfrak{S}}_{2} \in \mathscr{M}^{*}$. If $p \geqq 5$, we get $\subseteq=C_{\Im}\left(Z\left(\mathfrak{S}_{2}\right)\right) \cdot N_{\Im}\left(J\left(\mathfrak{S}_{2}\right)\right)$, and so $\subseteq \subseteq \mathfrak{M}$. This is false, and so $p=3$. Since $\Re$ has exponent 3 , $|\mathfrak{P}|=3$.

Let $\mathfrak{W}_{0}=V\left(\operatorname{ccl}_{\mathfrak{G}}(\mathfrak{F}) ; \mathfrak{S}_{2}\right)$. Then $\mathfrak{W}_{0} \neq 1$, since $\mathfrak{F} \subseteq \mathfrak{W}_{0}$, and $\mathfrak{W}_{0} \not \mathfrak{S}$, by maximality of $|\mathfrak{S} \cap \mathfrak{M}|_{2}$.

Let $\mathfrak{S}_{0}=\boldsymbol{O}_{2}(\mathfrak{S}), \mathfrak{S}_{1}=[\mathfrak{S}, \mathfrak{P}], \mathfrak{S}^{1}=\boldsymbol{C}_{\mathfrak{\Xi}_{0}}(\mathfrak{P})$, and let $\mathfrak{U}=\Omega_{1}\left(\boldsymbol{R}_{2}(\mathfrak{S})\right)$. We may assume that $\mathfrak{S}_{2} \subset \mathfrak{T}$, and so $\Omega_{1}(Z(\mathfrak{T})) \subseteq \mathfrak{H}$. Hence, $[\mathfrak{P}, \mathfrak{u}] \neq 1$, since $\Omega_{1}(Z(\mathfrak{T}))^{\ddagger} \subseteq \mathscr{F}_{\text {. }}$ Let $\mathfrak{H}_{1}$ be a minimal normal subgroup of $\subseteq$ with $\mathfrak{H}_{1} \subseteq[\mathfrak{P}, \mathfrak{u}] ; \mathfrak{u}_{1}$ exists since $\mathfrak{u} \subseteq Z\left(\mathfrak{S}_{0}\right)$, and $\mathfrak{S}_{0} \mathfrak{P} \triangleleft \Im_{\text {. }}$.

Choose $G \in \mathbb{B}$ such that $\mathfrak{F}^{G} \subseteq \mathfrak{S}_{2}, \mathfrak{F}^{G} \nsubseteq \mathfrak{S}_{0}$. Let $\mathfrak{F}_{1}=\mathfrak{F}^{G} \cap \mathfrak{S}_{0}$. Then $\left|\mathfrak{F}^{a}: \mathfrak{F}_{1}\right|=2$, as $\mathfrak{P}$ is cyclic, and $\mathfrak{H}_{1} \subseteq C\left(\mathfrak{F}_{1}\right)$. By Lemma 15.49, $\boldsymbol{C}\left(\mathfrak{\mho}_{1}\right) \subseteq \mathfrak{M}^{G}$, and by Lemma 15.50, $\left[\mathfrak{u}_{1}, \mathfrak{F}^{G}\right]^{\sharp} \cong \mathscr{J}^{G}$. Since $\boldsymbol{Z}\left(\mathfrak{S}_{2}\right) \cap$ $\left[\mathfrak{u}_{1}, \mathfrak{F}^{G}\right] \neq 1$, we get $\mathfrak{S}_{2} \subseteq \mathfrak{M}^{G}$. Since $N\left(\mathfrak{S}_{2}\right) \subseteq \mathfrak{M}$, maximality of $|\mathfrak{S} \cap \mathfrak{M}|_{2}$ forces $\mathfrak{M}=\mathfrak{M}^{a}$, whence $\mathfrak{\mho}=\mathfrak{F}^{G}$.

Clearly, $\mathfrak{S}_{0} \in \boldsymbol{U}^{*}(\mathfrak{P} ; 2)$, by maximality of $|\mathfrak{M} \cap \mathfrak{S}|_{2}$. Suppose $\boldsymbol{C}_{\varpi_{0}}(\mathfrak{P}) \neq 1$. By Lemma 15.57, we conclude that $N\left(\mathfrak{S}_{0}\right)$ is contained in some conjugate of $\mathfrak{M}$, and this conjugate is necessarily $\mathfrak{M}$ itself, by maximality of $|\mathfrak{S} \cap \mathfrak{M}|_{2}$. Hence, we conclude that $\boldsymbol{C}_{\varpi_{0}}(\mathfrak{P})=1$.

Since $\mathfrak{F} \triangleleft \mathfrak{S}_{2}$, we now get $\mathfrak{S}_{0}=\mathfrak{F}_{1} \times \mathfrak{F}_{1}^{P}$, where $\mathfrak{P}=\langle P\rangle$. This implies that $J\left(\Im_{2}\right) \triangleleft \Im$, which is false, since $\mathfrak{S}_{2}$ is not a $S_{2}$-subgroup of 8 . The proof is complete.

From now on, $\mathfrak{W}=V\left(\operatorname{ccl}_{\circledast}(\mathfrak{F}) ; \mathfrak{T}\right)$.

\section{LEMMA 15.59. $\quad N_{\mathfrak{M}}(\mathfrak{W})=\mathfrak{T}$.}

Proof. By Lemma $15.58, N(\mathfrak{W}) \nsubseteq \mathfrak{M}$, so by Lemma $15.29, N_{\mathfrak{m}}(\mathfrak{W})$ has cyclic $S_{3}$-subgroups, and so $\mathfrak{X}=\boldsymbol{N}_{\mathfrak{r}}(\mathfrak{W})$ has order 1 or 3 . Suppose $|\mathfrak{A}|=3$. First, suppose that $\mathfrak{A} \subseteq(\mathfrak{T} \mathfrak{X})^{\prime}$, and $\mathfrak{A} \subseteq Z(\mathfrak{R})$. In this case, we get $\mathfrak{N}=N(\mathfrak{W})=\mathfrak{I} \cdot C_{\mathfrak{R}}(\mathfrak{H})$, so that $\mathfrak{I}$ is permutable with $\left\langle\mathfrak{R}, C_{\mathfrak{r}}(\mathfrak{R})\right\rangle$, whence, by the usual argument, $\mathfrak{R} \subseteq \mathfrak{M}$, which is false. Suppose $\mathfrak{U} \subseteq(\mathfrak{I N})^{\prime}, \quad \mathfrak{X} \nsubseteq \boldsymbol{Z}(\mathfrak{R})$. In this case, $\mathfrak{N}=\mathfrak{I} \cdot \boldsymbol{C}_{\mathfrak{R}}(\mathfrak{A})$, and since $\boldsymbol{C}_{\mathfrak{f}}(\mathfrak{X})$ contains a four-group, we get $N(\mathfrak{R}) \subseteq \mathfrak{M}$ by Lemmas 14.20 and 14.22. Suppose finally that $\mathfrak{N} \nsubseteq \mathfrak{T}^{\prime}$. In this case, since $\mathfrak{R} \supseteqq \mathfrak{M}^{\prime}$, we conclude that $\mathfrak{A}=\mathfrak{R}^{\prime}, l_{3}(\mathfrak{M})=1$, and elements of $\mathfrak{T}$ induce automorphisms of $\mathfrak{X}=\mathfrak{S} \mathfrak{R} / \mathfrak{S} \mathfrak{R}^{\prime}$ of determinant 1 . Since $\mathfrak{X}=\mathfrak{R}^{\prime}$, we have $|\mathfrak{B}, \mathfrak{A}|=1$, so $\mathfrak{X}$ centralizes $Z(\mathfrak{T})$. By Lemma 14.29, $S_{3}$-subgroups of $\mathfrak{R}$ are cyclic, and

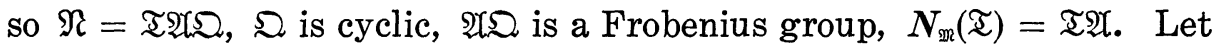
$\mathfrak{P}$ be a subgroup of $\mathfrak{D}$ of prime order, and let $\mathfrak{N}_{0}=\mathfrak{I} \mathfrak{N} \mathfrak{P}_{\mathrm{P}}$. Let $\mathfrak{U}$ be a minimal normal subgroup of $\mathfrak{N}_{0}$. Then $\boldsymbol{C}_{\mathfrak{R}_{0}}(\mathfrak{U})=\mathfrak{N}_{1}=\boldsymbol{O}_{2}\left(\mathfrak{N}_{0}\right)$, and $\mathfrak{N}_{0} / \mathfrak{N}_{1}$ is a Frobenius group of order p. $3.2^{a}, a \geqq 1$. It follows that $\mathfrak{A}$ does not centralize $\boldsymbol{Z}(\mathfrak{I})$. This contradiction completes the proof.

Let

$$
\begin{aligned}
\mathscr{K} & =\left\{\mathfrak{Y} \mid \mathfrak{Y} \in \operatorname{ccl}_{\mathfrak{S}}(\mathfrak{F}), \quad \mathfrak{Y} \subseteq \mathfrak{M}, \mathfrak{Y} \varsubsetneqq \mathscr{S}\right\} \\
\mathscr{K}(\mathfrak{I}) & =\{\mathfrak{Y} \in \mathscr{K} \mid \mathfrak{Y} \subseteq \mathfrak{T}\} .
\end{aligned}
$$


Thus, $\mathscr{K}(\mathfrak{I}) \neq \varnothing$.

If $\mathfrak{Y}=\mathfrak{F}^{G} \subseteq \mathfrak{M}$, then $\boldsymbol{C}_{\mathfrak{Y}}(\mathfrak{F})$ contains a hyperplane of $\mathfrak{Y}$, and so $\mathfrak{F} \subseteq \mathfrak{M}^{a}$, whence $\left[\mathfrak{F}^{G}, \mathfrak{F}\right] \subseteq\left[\cap \cap \mathscr{J}^{a}\right.$. We conclude that

$$
\mathfrak{W} \subseteq C(\mathfrak{S}) \text {. }
$$

Since $\mathfrak{M}=\mathfrak{M}_{0} \cdot N_{\mathfrak{M}}(\mathfrak{W})$, Lemma 15.59 implies that $\mathfrak{M}=\mathfrak{M}_{0} \mathfrak{T}$, and so

$$
\Re \leqq C(\mathfrak{S}) \text {. }
$$

Choose $\mathfrak{Y}=\mathfrak{F}^{a} \in \mathscr{K}(\mathfrak{T})$, and let $\mathfrak{Y}_{0}=\mathfrak{Y} \cap \mathfrak{K}$. Let $\mathfrak{u}$ be a complement to $\mathfrak{V}_{0}$ in $\mathfrak{Y}$. Then $\mathfrak{U}$ acts faithfully on $\boldsymbol{O}_{3^{\prime}, 3}(\mathfrak{M}) / \mathfrak{F}$, and so $2 \leqq$ $|\mathfrak{u}| \leqq 4$. We argue that $|\mathfrak{u}|=2$. Suppose $|\mathfrak{u}|=4$. In this case, $\Re_{0}$ has a subgroup $\subseteq$ of order 9 such that $\mathfrak{U}$ acts faithfully on $\mathfrak{C} \subseteq / \mathfrak{K}$.

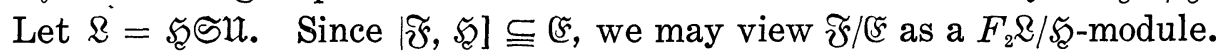
Let $\mathfrak{S}=\mathfrak{S}_{1} \times \mathfrak{S}_{2}$, where $\left|\mathfrak{S}_{i}\right|=3$, and $\mathfrak{S C}_{i} \triangleleft \mathfrak{R}$. Let $\mathfrak{U}_{3-i}=\boldsymbol{C}_{\mathfrak{n}}\left(\mathfrak{S C S}_{i} / \mathfrak{S}_{\mathfrak{c}}\right)$, so that $\mathfrak{U}=\mathfrak{U}_{1} \times \mathfrak{U}_{2}$. Let $\mathfrak{R}_{i}=\mathfrak{S} \mathfrak{S}_{i} \mathfrak{U}_{i}$, so that $\mathfrak{L} / \mathfrak{S}=\mathfrak{L}_{1} / \mathfrak{S} \times \mathfrak{R}_{2} / \mathfrak{S}$, and $\mathfrak{l}_{i} / \mathfrak{S} \cong \sum_{3}$.

Set $\overline{\mathscr{\mho}}=\mathfrak{F} / \mathfrak{F}$, and let $\overline{\mathscr{\mho}}_{1}=\left[\overline{\mathscr{\mho}}, \mathfrak{S}_{1}\right] \cap\left[\overline{\mathscr{\mho}}, \mathfrak{S}_{2}\right]$. We will show that $\overline{\mathscr{\mho}}_{1}=1$. Suppose false. In any case, $\overline{\mathscr{F}}_{1}$ admits $\mathbb{R}$, and so we can choose an irreducible $F_{2} \mathrm{R}$-submodule $\overline{\mathscr{F}}_{2}$ of $\overline{\mathscr{F}}_{1}$. It follows that $\left|\overline{\mathscr{F}}_{2}\right|=16$, and $\overline{\mathscr{\mho}}_{2}$ is a free $F_{2} \mathfrak{l}$-module. Let $\overline{\mathscr{\mho}}_{3}=3 / \mathbb{F}$ be the unique $\mathfrak{l}$-admissible subgroup of $\bar{\mho}_{2}$ of order 8 , and let $\tilde{Z} / \mathbb{F}$ be the unique $\mathfrak{U}$-admissible subgroup of $\widetilde{F}_{2}$ of order 2 . Then for each $Z$ in 3 , we have [Y, $\left.Z\right] \subseteq \tilde{8}$, and so $\boldsymbol{C}_{\mathfrak{y}}(Z)$ is of index at most $2|\mho|$ in $\mathfrak{Y}$, whence $Z \in C\left(\boldsymbol{C}_{\mathfrak{y}}(Z)\right) \subseteq \mathfrak{M}^{G}$. Now $|3|=8|\&|$, and we can choose a four-group $3^{*}$ such that $3=$ $3^{*} \times \widetilde{3}$. By the preceding argument, we have $3^{*} \subseteq \mathfrak{M}^{a}$. Since $\mathfrak{U}$ acts faithfully on 3 , we get $C\left(\mathfrak{\mho}^{G}\right) \cap 3^{*}=1$, so by Lemma 15.54 , we get that $\left[\mathfrak{Z}^{*}, \mathfrak{F}^{G}\right] \cap \mathscr{J}^{a} \neq \varnothing$. Hence, $\widetilde{F} \cap \mathscr{J}^{a} \neq \varnothing$, and so $\mathfrak{F} \subseteq \mathfrak{M}^{a}$. This implies that $\left[\Re, \mathfrak{\mho}^{G}, \mathfrak{\mho}^{G}\right]=1$, and so the module $\bar{\mho}_{2}$ does not exist. Hence, $\bar{\mho}_{1}=1$.

We next argue that $\subseteq$ has no non trivial fixed points on $\bar{\mho}$. This is clear if $\mathfrak{S}=\Re_{1}$, since $\overline{\mathscr{F}}$ is a chief factor of $\mathfrak{M}$. Suppose $\mathfrak{S} \subset \mathfrak{R}_{1}$. In this case, $\mathfrak{R}_{1}^{\prime} \subseteq \mathfrak{S}$, and $\mathfrak{S}_{\mathfrak{c}} \mathfrak{R}_{1}^{\prime} \triangleleft \mathfrak{M}$, whence $\mathfrak{R}_{1}^{\prime}$ has no fixed points on $\mathfrak{\mho}$. Since $\Re_{1}^{\prime} \subseteq \mathfrak{S}$, we conclude in any case that $\mathfrak{S}$ has no

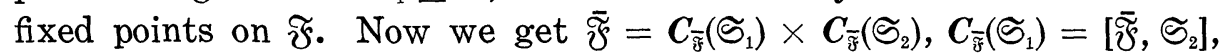
$C_{\tilde{\widetilde{\gamma}}}\left(\mathfrak{S}_{2}\right)=\left[\overline{\mathscr{\mho}}, \mathfrak{S}_{1}\right]$. Thus, $\mathfrak{S}_{1}, \mathfrak{S}_{2}$ are the only subgroups of $\mathfrak{S}$ of order 3 which have fixed points on $\mathfrak{F}$, and since $\mathfrak{S}$ acts faithfully on $\mathfrak{F}$, we have $C_{\Re}\left(\Im_{i}\right) \neq 1$. Hence, $\mathfrak{S}_{1}, \mathfrak{S}_{2}$ are central subgroups of $\Re$, and so $\mathfrak{S}=\Re$. This implies that $|\mathfrak{F} / \mathfrak{F}|=16$, and $\mathfrak{F}=\mathfrak{F} \times \mathfrak{F}_{1} \times \mathfrak{F}_{2}$, where $\mathfrak{\mho}_{1}=\left[\mathfrak{F}, \mathfrak{S}_{2}\right], \mathfrak{F}_{2}=\left[\mathfrak{F}, \mathfrak{S}_{1}\right], \widetilde{F}_{1}, \widetilde{\mho}_{2}$ being four-groups. Also, $\mathfrak{F}_{\mathfrak{F}_{1}}$, $\mathfrak{5} \mathfrak{F}_{2}$ are normalized by $\mathfrak{F}^{G}$. If $F \in \mathcal{E} \mathfrak{F}_{i}$, then $C(F) \cap \mathfrak{F}^{G}$ is of index at most $2|\mathfrak{F}|$ in $\mathfrak{F}^{G}$, and so $\mathfrak{F}_{i} \subseteq \mathfrak{M}^{a}$. So $\mathfrak{F} \subseteq \mathfrak{M}^{G}$. Choose $\mathfrak{F}^{i}$ to be the unique hyperplane of $\mathfrak{F}^{\mathfrak{F}_{i}}$ which contains $\mathfrak{F}$ and admits $\mathfrak{F}^{G}=\mathfrak{Y}$, and then choose $F_{i} \in \mathfrak{F}_{i}-\mathfrak{F}^{i}$. Let $\mathfrak{u}^{*}=\left\langle F_{1}, F_{2}\right\rangle$. Then $\mathfrak{u}^{*} \cap \boldsymbol{C}\left(\mathfrak{F}^{G}\right)=1$, and so by Lemma 15.54, $\left[\mathfrak{F}^{G}, \mathfrak{U}^{*}\right] \cap \mathscr{J}^{G} \neq \varnothing$. Since $\mathfrak{S}_{i}$ centralizes 


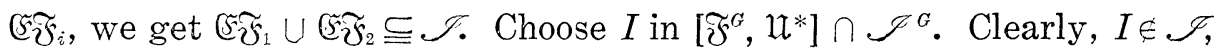
since $\mathscr{F}$ is a T.I. set in $\mathfrak{S}$, and $\mathscr{F} \neq \mathscr{J}^{G}$. Since $\left[\mathfrak{F}^{G}, \mathfrak{U}^{*}\right] \subseteq \mathfrak{F}^{1} \mathfrak{F}^{2}$, we have $I=I_{1} I_{2} E$, where $E \in \mathfrak{F}$, and $I_{i} \in \mathfrak{F}_{i} \cap \mathfrak{F}^{i}$. Since $I \notin \mathscr{F}$, we have $I_{i} \neq 1, i=1,2$, and so $\left\langle I_{i}\right\rangle=\widetilde{\mho}_{i} \cap \widetilde{\mho}^{i}$.

Next, observe that $\mathfrak{Y}_{0} \mathfrak{U}_{1}$ is a hyperplane of $\mathfrak{Y}$ which is 1 on $\mathfrak{E}_{\mathfrak{F}_{1}} / \mathfrak{F}$. If $\mathfrak{Y}_{0} \mathfrak{U}_{1}$ centralizes $\left[\mathfrak{F} \widetilde{F}_{1}\right.$, we get that $\left[\widetilde{F}_{1}, \mathfrak{F}^{G}\right]^{*} \leqq \mathscr{J}^{G}$, which is false,

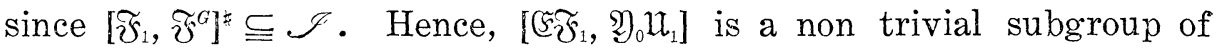
5. Suppose $|\mathfrak{F}|=2$, so that $|\widetilde{\mho}|=2^{5}$. In this case, we get that $\left|\mathfrak{F} \cap \mathfrak{F}^{G}\right|=2^{3}$, and so $\mathfrak{F}^{G}=\mathfrak{U} \times \mathfrak{F} \cap \mathfrak{F}^{G}$, and $\mathfrak{F} \cap \mathfrak{F}^{G}$, contains precisely 5 elements of $\mathscr{F}$. If $\widetilde{F} \cap \mathfrak{S C}^{G}=\mathfrak{F} \cap \mathfrak{F}^{G}$, then by symmetry, $\mathfrak{F} \cap \mathfrak{F}^{G}$ contains precisely 5 elements of $\mathscr{F}^{G}$, which is impossible since $\left|\mathfrak{F} \cap \mathfrak{F}^{G=}\right|=7$, and $\mathscr{F} \cap \mathscr{J}^{G}=\varnothing$. If $\mathfrak{F}^{G} \cap \mathfrak{F} \supset \mathfrak{F}^{G} \cap \widetilde{F}$, and $F \in \mathfrak{S C}^{G} \cap \widetilde{F}-$ $\mathfrak{F}^{G} \cap \widetilde{F}$, then $\left[\widetilde{F}, \widetilde{F}^{G}\right]$ contains an element of $\left(\mathfrak{E} \widetilde{F}_{1} \cup \mathfrak{F}^{2} \widetilde{F}_{2}\right)^{ \pm}$, which is also impossible since $\mathfrak{F}^{\sharp} \subseteq \mathscr{F}$, while $\left[\widetilde{F}_{i}, \widetilde{F}^{G}\right]^{\ddagger} \subseteq \mathscr{I}^{G}$. We conclude that $|\mathfrak{s}|=4$.

We argue that $\mathfrak{S}^{G} \cap \widetilde{\mho}=\left(\mathfrak{F} \cdot\left(\widetilde{F} \cap \widetilde{\mho}^{G}\right)\right.$. In any case, $\widetilde{F} \cdot\left(\mathfrak{F} \cap \widetilde{F}^{G}\right)$ is contained in $\mathfrak{S C}^{G}$, since $\widetilde{F} \cap \mathfrak{F}^{G}$ visibly is, and since $\mathbb{F}$ centralizes $\widetilde{F}^{G}$. If $\mathfrak{F} \cdot\left(\mathfrak{F} \cap \mathfrak{F}^{G}\right) \subset \mathfrak{F} \cap \mathfrak{S}^{G}$, and $F \in \mathfrak{F} \cap \mathfrak{S}^{G}-\mathfrak{G}\left(\widetilde{F} \cap \mathfrak{F}^{G}\right)$, we get $\left[F, \mathfrak{F}^{G}\right]^{*} \subseteq$ $\mathscr{J}^{G}$, against $\left[F, \widetilde{F}^{G}\right] \cap \mathscr{J} \neq \varnothing$. So $\mathfrak{S}^{G} \cap \widetilde{F}=\mathscr{F}\left(\widetilde{F} \cap \mathfrak{F}^{G}\right)$ is of index 4 in $\mathfrak{F}_{\text {. If }} \mathfrak{F} \cap \mathfrak{F}^{G}$ has order 8 , then $\widetilde{F} \cap \mathfrak{F}^{G}$ has at least 5 elements of $\mathscr{F}$, and by symmetry, $\widetilde{F} \cap \widetilde{F}^{G}$ has at least 5 elements of $\mathscr{F}^{a}$. This violates $\mathscr{I} \cap \mathscr{I}^{a}=\varnothing$. If $\widetilde{F} \cap \mathfrak{\mho}^{G}$ has order 16 , then $\widetilde{F} \cap \mathfrak{F}^{G}$ contains at least 11 elements of $\mathscr{J}$, so by symmetry, has at least 11 elements of $\mathscr{I}^{a}$. This also violates $\mathscr{I} \cap \mathscr{I}^{G}=\varnothing$. We conclude that $|\mathfrak{u}|=2$. Let $\mathfrak{u}=\langle U\rangle$.

If $F \in \widetilde{F}$, then $\left[F, \mathfrak{F}^{G} \cap \mathfrak{S}_{\mathcal{E}}\right] \subseteq \mathfrak{F}$, and $C(F) \cap \mathfrak{F}^{G} \cap \mathfrak{F}$ has index at most $|\mathfrak{F}|$ in $\mathfrak{F}^{G} \cap \mathfrak{S}$. Hence, $C(F) \cap \mathfrak{F}^{G}$ has index at most $2|\mathfrak{F}|$ in $\mathfrak{F}^{G}$, whence $F \in C\left(\mathfrak{F}^{G} \cap C(F)\right) \subseteq \mathfrak{M}^{G}$. Thus, $\mathfrak{F} \subseteq \mathfrak{M}^{G}$. By symmetry, $\left|\widetilde{F}: \widetilde{\mho} \cap \mathfrak{F}^{G}\right| \leqq 2$. Since $\left[U, \widetilde{F} \cap \mathfrak{S}^{G}\right] \subseteq \mathfrak{F}^{G}$, it follows that $|[\widetilde{\mho}, U]| \leqq$ $2|\mathfrak{S}|$. In other words, $U \in \mathfrak{Q}_{1} \cup \mathfrak{T}_{2} \cup \mathfrak{N}_{3}$.

Case 1. $|\Re|=3^{2}$ and $N_{\mathfrak{m}}(\Re)$ permutes transitively the subgroups of $\Re$ of order 3 .

In this case, we get $|\widetilde{\mho} / \mathfrak{F}|=2^{3}$, and $\widetilde{F}=\mathfrak{F} \times \widetilde{\mho}_{1} \times \widetilde{F}_{2} \times \widetilde{\mho}_{3} \times \widetilde{\Re}_{4}$, where $\widetilde{\mho}_{i}$ are four-groups which admit $\Re$, and $\Re^{i}=C_{\Re}\left(\mathfrak{F}_{i}\right)$ are the subgroups of $\Re$ of order 3 . We may assume that $U$ inverts $\Re^{1}$. Since $\left|\left[\mathfrak{F}, \Re^{1}\right]\right|=2^{6}$, we get $|[\mathfrak{F}, U]| \leqq 2^{3}$. Since $U \in \mathfrak{A}_{f}$ with $f \leqq 3$, we conclude that $|[\widetilde{\mho}, U]|=2^{3}$, and since $\widetilde{\mho} \cap \mathfrak{S}^{G}$ is of index at most 2 in $\widetilde{F}$, we then get that $\left|\left[\widetilde{F} \cap \mathfrak{S C}^{G}, U\right]\right| \geqq 2^{2}$. Since $\left[\widetilde{F} \cap \mathfrak{S}_{\mathfrak{C}}{ }^{G}, U\right] \leqq \mathfrak{F}^{G}$, we conclude that $|\mathfrak{F}|=4$, and that $\left[\widetilde{F} \cap \mathfrak{S}^{G}, U\right]=\left[\mathfrak{F}^{G} \subset[\widetilde{F}, U]\right.$. Since $|[\mathfrak{F}, U]|=2^{3}$, it follows that $\mathfrak{F} \cap \mathfrak{S}^{G}$ is a hyperplane of $\mathfrak{F}$. Also, $U$ does not invert $\mathfrak{F} \Re / \mathfrak{F}$ since $|\widetilde{F} / \mathfrak{F}|=2^{s}$. Let $\Re^{i}$ be the unique subgroup of $\Re$ of order 3 such that $U$ centralizes $\mathfrak{S C}_{\mathfrak{C}} \Re^{i} / \mathfrak{S}$. Then $\Re^{i}$ normalizes $[\mathfrak{F}, U] \mathfrak{F}$, and so normalizes $([\widetilde{\mho}, U] \mathfrak{F}) \cap[\widetilde{F}, \Re]=[\mathfrak{F}, U]$. Thus, as 
$\mathfrak{F}^{G} \subset[\mathfrak{F}, U]$, and $[\mathfrak{F}, U]=8$, we get $\mathfrak{F}^{G} \cap \mathfrak{F}^{G R} \neq 1$, where $R$ is a generater for $\mathfrak{R}^{i}$. Since $\mathfrak{F}^{a}$ is a T.I. set in (S), we get $R \in N\left(\mathfrak{F}^{G}\right)=\mathfrak{M}^{a}$. Since $[\Re, \mathfrak{F}]=1$, so also $\left[R, \mathfrak{F}^{G}\right]=1$. Since $|[\mathfrak{F}, U]|=8$, this implies

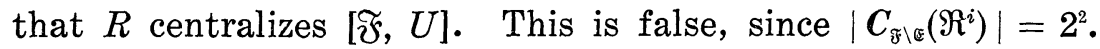

Case 2. $|\Re|=3^{2}$, and $N_{\mathfrak{n}}(\Re)$ does not permute transitively the subgroups of $\mathfrak{R}$ of order 3 .

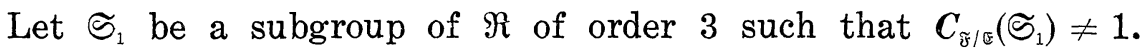
Since $|\mathfrak{F} / \mathfrak{F}|>4, N_{\mathfrak{m}}(\Re)$ does not normalize $\mathfrak{S}_{1}$, and so $\Re=\mathfrak{S}_{1} \times \mathfrak{S}_{2}$, where $\left\{\mathfrak{S}_{1}, \mathfrak{S}_{2}\right\}$ is an orbit under $\boldsymbol{N}_{\mathfrak{n}}(\Re)$ of the subgroups of $\Re$ of order $\Re$, 3. Hence, $\mathfrak{F}=\mathfrak{F}_{1} \times \mathfrak{F}_{2} \times \mathfrak{F}$, where $\mathfrak{\mho}_{i}$ are four-groups which admit and $\widetilde{\mho}_{i} \subseteq C\left(\Im_{i}\right)$.

Let $\tilde{\mho}=\left\{E F_{1} F_{2}, E \in \mathfrak{F}, F_{i} \in \widetilde{F}_{i}^{*}\right\}$. Thus, $|\tilde{\mho}|=9|\mathfrak{F}|$, and $\tilde{\mho}$ admits $\mathfrak{F} \Re$. We argue that $\tilde{\mathfrak{F}}$ is a conjugacy class of $\mathfrak{S} \Re$. Namely, let

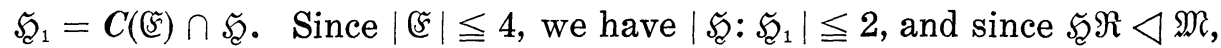
we get that $\mathfrak{M}=\mathfrak{S}_{1} \cdot N_{\mathfrak{M}}(\Re)$. Let $\mathfrak{F}^{*}=\widetilde{\mho} \cap[\mathfrak{F}, \mathfrak{R}]$, so that $\left|\mathfrak{F}^{*}\right|=9$, and $\Re$ is transitive on $\mathfrak{F}^{*}$. Choose $F \in \mathfrak{F}^{*}$. Then $\mathfrak{H}_{1}$ normalizes $F \mathfrak{F}$, so it suffices to show that $\left[F, \mathfrak{S}_{1}\right]=\mathbb{F}$. Suppose $\left[F, \mathscr{S}_{1}\right]=\mathfrak{F}_{0} \subset \mathbb{F}$. Since $\mathbb{F} \subseteq Z\left(\mathfrak{S}_{1}\right)$, we get $\mathfrak{F}_{0} \triangleleft \mathfrak{F}_{1}$. Since $\left[\mathfrak{F}_{0}, \mathfrak{R}\right]=1$, we get next that $\left[F^{\Re}, \mathfrak{S}_{1}\right]=\mathfrak{F}_{0}$. Since $F \in \mathfrak{F}^{*}, \mathfrak{F}^{\Re}=[\mathfrak{F}, \Re]$, and since $N_{\mathfrak{M}}(\Re)$ normalizes $\mathfrak{S}_{1}$ and $[\mathfrak{F}, \mathfrak{R}]$, we get $\mathfrak{F}_{0} \subseteq Z(\mathfrak{Z})$. Now $\mathfrak{F}_{0}[\mathfrak{F}, \mathfrak{R}]$ admits $\mathfrak{F}_{1}$ and $N_{\mathfrak{M}}(\Re)$. Since $\mathfrak{M}=\mathfrak{S}_{1} \cdot N_{\mathfrak{M}}(\mathfrak{R})$, and $\mathfrak{F}_{0}[\mathfrak{F}, \mathfrak{R}] \subset \mathfrak{F},\left|\mathfrak{F}_{0}[\mathfrak{F}, \mathfrak{R}]\right| \geqq 8$, the minimality of $\mathfrak{F}$ is violated. So

\section{$\tilde{\mathfrak{F}}$ is a conjugacy class of $\mathfrak{S} \Re$.}

Note that $\mathfrak{F}-\tilde{\mho} \subseteq \mathscr{I}$, and so $\mathfrak{F} \cap \mathscr{I}^{a} \subseteq \widetilde{\mho}$. We argue that $\mathscr{J}^{G} \cap\left[\mathfrak{F}, \mathfrak{F}^{G}\right] \neq \varnothing$. Namely, $\mathfrak{F} \cap \mathfrak{S}^{G}$ is of index at most 2 in $\mathfrak{F}$, and $\left[\mathfrak{F} \cap \mathfrak{S}^{G}, \mathfrak{F}^{G}\right] \subseteq \mathfrak{F} \cap \mathfrak{F}^{G}$. Since $\mathfrak{F}^{G \sharp} \subseteq \mathscr{I}^{G}$, we are done if $\left[\mathfrak{F} \cap \mathfrak{S}^{G}, \mathfrak{F}^{G}\right] \neq 1$. So suppose $\left[\mathfrak{F} \cap \mathfrak{S}^{G}, \mathfrak{F}^{G}\right]=1$. In this case, $\mathfrak{F}=\mathfrak{F} \cap \mathfrak{S}^{G} \times\langle F\rangle$, where $F \neq 1$. Also, of course, $\mathfrak{F}^{G}=\mathfrak{Y}_{0} \times \mathfrak{H}$. Set $J=[U, F]$. Since $U \notin \mathfrak{F}$,

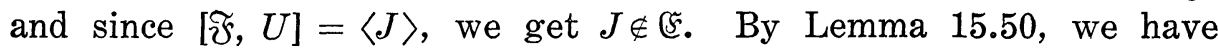
$J \in \mathscr{F}$. If in addition, we have $\left[\mathfrak{F}^{G} \cap \mathfrak{F}, \mathfrak{F}\right]=1$, then we can apply Lemma 15.50 to $F$ acting on $\mathfrak{F}^{G}$, and get $J \in \mathscr{J}^{G}$. This is false, and so $\left[\mathfrak{F}^{G} \cap \mathfrak{S}, \mathfrak{F}\right] \neq 1$. Choose $E \in\left[\mathfrak{F}^{G} \cap \mathfrak{S}, \mathfrak{F}^{*}\right.$. Thus, $E \in \mathfrak{F}^{\#} \subseteq \mathscr{F}$. Thus, we have the following situation: $E, J$ are elements of $\mathscr{J} \cap\left[\mathfrak{F}_{,} \mathfrak{F}^{G}\right]$, and $E \in \mathfrak{F}, J \in \mathfrak{F}-\mathfrak{F}$. Since $I$ is a T.I. set in $\mathbb{S}$, and $\mathbb{F} \triangleleft \mathfrak{M}$, it follows that $E$ and $J$ are not $\$$-conjugate. Hence, $\left|\{E, J\} \cap \tilde{\mho}^{G}\right| \leqq 1$. But $\{E, J\} \subseteq \mathfrak{F}^{G}$, and $\mathfrak{F}^{G}-\tilde{\mathscr{F}}^{G} \subseteq \mathscr{J}^{a}$. Hence, $\{E, J\} \cap \mathscr{J}^{G} \neq \varnothing$, which is false, since $\mathscr{J} \cap \mathscr{J}^{G}=\varnothing$. We have thus shown that $\left[\mathfrak{F}, \mathfrak{\mho}^{G}\right] \cap$ $\mathscr{I}^{G} \neq \varnothing$.

Let $\mathfrak{Y}=\mathfrak{F}(1)=\mathfrak{F}^{G}, \mathfrak{F}(2), \cdots, \mathfrak{F}(m)$ be all the elements of $\mathscr{K}(\mathfrak{T})$. For each $i$, choose $G(i) \in \mathbb{S}$ such that $\mathfrak{F}(i)=\mathfrak{F}^{G(i)}$. Then choose $F(i)$ in $[\widetilde{F}, \mathfrak{F}(i)] \cap \mathscr{J}^{G(i)}$. Set $F=F(1)$, and choose $X(i) \in \mathfrak{S} \Re$ with $F(i)^{X(i)}=F$. These elements $X(i)$ exist, since $[\widetilde{\mho}, \widetilde{F}(i)] \cap \mathscr{J}^{G(i)} \subseteq \widetilde{\mho}$. Thus, for each 
$i, F$ is in $\mathscr{J}^{G(i) X(i)} \cap \mathscr{I}^{G}$. Hence, $\mathfrak{F}^{G(i) X(i)}=\mathfrak{F}^{G}=\mathfrak{Y}=\mathfrak{F}^{(i)^{X(i)}}$, and so $\mathfrak{F}(i) \subseteq \mathfrak{S} \mathfrak{Y} \Re$ for all $i$. Thus, if $\mathfrak{F}^{*} \in \operatorname{ccl}_{\circledast}(\mathfrak{F})$, and $\mathfrak{F}^{*} \subseteq \mathfrak{I}$, then either $\mathfrak{F}^{*} \subseteq \mathfrak{F}$, or $\mathfrak{F}^{*}$ is one of our $\mathfrak{F}(i)$. We conclude that $\mathfrak{B} \subseteq \mathfrak{S} \mathfrak{Y} \Re$, and so $\mathfrak{W} \leqq \mathfrak{S}_{\mathfrak{Y}} \mathfrak{R} \cap \mathfrak{I}=\mathfrak{S}_{\mathfrak{Y}} \mathfrak{Y}$. Since $\boldsymbol{N}_{\mathfrak{M}}(\mathfrak{W})=\mathfrak{I}$, it follows that $U$ inverts $\mathfrak{K} \Re / \mathfrak{K}$. Hence, we get that $U$ inverts some $S_{3}$-subgroup of $\mathfrak{F} \Re$, which we may assume is $\Re$. In this case, $U$ normalizes both of the fourgroups $\widetilde{\mho}_{1}, \mathfrak{\mho}_{2}$, and if $\mathfrak{\mho}_{i}^{*}=\left[\mathfrak{\mho}_{i}, U\right]$, then $\left|\mathfrak{\mho}_{i}^{*}\right|=2$, and $C_{\mathfrak{F}}(U)=\mathfrak{F} \times$ $\mathfrak{F}_{1}^{*} \times \mathfrak{F}_{2}^{*}$. Since $\left[\mathfrak{F}, \mathfrak{F}^{G}\right] \subseteq \mathfrak{F}^{G}$, it follows that $\mathfrak{F} \cap \mathfrak{F}^{G}=\mathfrak{F}_{1}^{*} \times \mathfrak{F}_{2}^{*} \times$

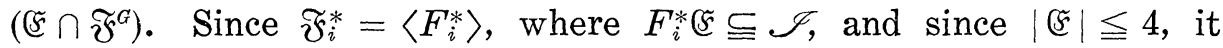
follows that $\left|\mathfrak{F} \cap \mathfrak{F}^{G}\right|=4$, 8, or 16 , and in the three cases, we have $\left|\mathfrak{F} \cap \mathfrak{F}^{G} \cap \mathscr{J}\right| \geqq 2,5$, 11, respectively. If $\mathfrak{F} \subseteq \mathfrak{S}^{G}$, then $\left[\mathfrak{F}, \mathfrak{F}^{G}\right] \leqq \mathfrak{F}^{G}$, which gives $F_{1}^{*} \in \mathscr{F}^{G} \cap \mathscr{G}$, which is false. Hence, symmetry implies that $\mathscr{I} \cap \mathscr{J}^{G} \neq \varnothing$. This completes the analysis in case $|\Re|=3^{2}$.

Case 3. $|\mathfrak{R}|=3^{3}$ and $l_{3}(\mathfrak{M})=1$.

Let $\mathfrak{P}$ be a subgroup of $\mathfrak{M}$ of order 3 inverted by $U$. Thus, $|[\mathfrak{F}, \mathfrak{P}]| \leqq 2^{6}$. Since $\mathfrak{S} \Re \triangleleft \mathfrak{M}$, it follows that $\mathfrak{F} / \mathfrak{F}$ is a direct sum of irreducible $F_{2} \Re$-modules, on each of which $\Re^{\prime}$ acts faithfully. Since $|[\widetilde{F} / \mathfrak{F}, \mathfrak{P}]| \leqq 2^{6}$, we get $\left.\mid \mathfrak{F} / \mathfrak{F}\right]=2^{6}$, and if $\mathfrak{P}$ is a non central subgroup of $\Re$ of order 3 , then $|[\mathfrak{F} / \mathfrak{F}, \mathfrak{P}]|=2^{4}$.

Since $\boldsymbol{N}_{\mathfrak{m}}(\mathfrak{W})=\mathfrak{T}$, we can suppose $\mathfrak{Y}$ has been chosen so that $\mathfrak{Y}$ does not centralize $\mathfrak{S E R}_{\mathfrak{C}} / \mathfrak{F}$, and we can then suppose that $U$ inverts $\Re^{\prime}$. Let $\mathfrak{F}=\mathfrak{F} \times \mathfrak{F}_{1}$, where $\mathfrak{F}_{1}=\left[\mathfrak{F}, \mathfrak{R}^{\prime}\right]$ is of order $2^{6}$. Thus, $\left|\left[\widetilde{\mho}_{1}, \mathfrak{u}\right]\right|=2^{3}$, and $\left[\widetilde{\mho}_{1}, U\right]=[\mathfrak{F}, U] \subseteq \widetilde{\mho}_{1}$. Since $|\Re|=3^{3}$, $\Re$ has a subgroup $\mathfrak{S}$ of order 3 such that $U$ centralizes $\mathfrak{S C S} / \mathfrak{S}$. Thus, $\mathfrak{S}$ normalizes $\mathfrak{s}[\mathfrak{F}, U]$, and so normalizes $\mathfrak{F}_{1} \cap(\mathfrak{F}[\mathfrak{F}, U])=[\mathfrak{F}, U]$. Since $\mathfrak{F} \cap \mathfrak{S}^{G}$ is of index at most 2 in $\mathfrak{F}$, we get $\left|\left[\mathfrak{F} \cap \mathfrak{S}^{G}, U\right]\right| \geqq 2^{2}$, and so $\left[\mathfrak{F} \cap \mathfrak{S}^{G}, U\right]=\mathfrak{F}^{G}$ is a four-group. Let $\mathfrak{S}=\langle S\rangle$. Since $|[\mathfrak{F}, U]|=8$, and $[\mathfrak{F}, U]$ admits $\subseteq$, it follows that $\mathfrak{F}^{\mathscr{G}} \cap \mathfrak{F}^{G S} \neq 1$. Hence, $S$ normalizes

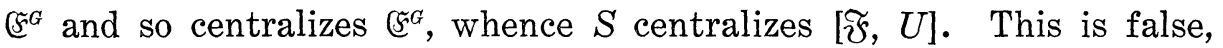
since $\left|\left[\mathfrak{\mho}_{1}, \mathfrak{S}\right]\right|=2^{4}$.

\section{Case 4. $|\Re|=3^{3}$ and $l_{3}(\mathfrak{M})>1$.}

In this case, $\mathfrak{M} / \mathfrak{F}$ is a familiar group: a splitting extension of an elementary group $\Re_{1} \mathfrak{E} / \mathfrak{S}$ of order $3^{2}$, by $G L(2,3)$. Let $\mathfrak{I}_{1} / \mathfrak{S}$ be the center of $\mathfrak{I} / \mathfrak{F}$. We first argue that $\mathfrak{Y} \varsubsetneqq \mathfrak{I}_{1}$. For if $\mathfrak{Y} \subseteq \mathfrak{I}_{1}$, then $U$ inverts $\mathscr{S C}_{\mathfrak{C}} / \mathfrak{F}_{\mathfrak{C}}$, and so $|[\mathfrak{F}, U]| \geqq 2^{4}$, against $U \in \mathfrak{A}_{f}, f \leqq 3$.

Let $\mathfrak{P}$ be a subgroup of $\Re_{1} \mathscr{F}$ of order 3 inverted by $U$. We choose notation so that $\mathfrak{B} \subseteq \Re_{1}$. Now $\mathfrak{F}=\mathbb{F} \times \mathfrak{F}_{1} \times \cdots \times \mathfrak{F}_{4}$, where $\mathfrak{F}_{i}=\left[C_{\Re}\left(\Re^{i}\right)\right.$, $\Re$, and $\Re^{1}, \cdots, \Re^{4}$ are subgroups of $\Re_{1}$ of order 3 . Also, $\widetilde{F}_{1}, \cdots, \mathfrak{F}_{4}$ are permuted transitively by $N_{\mathfrak{M}}\left(\Re_{1}\right)$. It follows that if $\left|\widetilde{\Im}_{i}\right|=2^{2 k}$, then $\left|\left[\widetilde{F}, \Re^{i}\right]\right|=2^{6 k}$. We may assume that $\mathfrak{P}=\Re^{1}$. Since $U \in \mathfrak{X}_{f}$, and $f \leqq 3$, we get $k=1,|\mathfrak{F} / \mathscr{\mho}|=2^{8}$. Since we now get $f=3$, 
we conclude that $1 \subset \mathfrak{F}^{a} \subset[\mathfrak{F}, U],|\mathfrak{F}|=4$. Let $\mathfrak{Q}$ be the unique subgroup of $\Re_{1}$ of order 3 such that $U$ centralizes $\mathfrak{F} \Omega / \mathscr{F}$. Thus, as before, $\mathfrak{Q}$ normalizes $[\mathfrak{F}, U]=(\mathfrak{F}[\mathfrak{F}, U]) \cap\left[\Im, \Re^{1}\right]$, and so $\mathfrak{F}^{G} \cap \xi^{G Q} \neq 1$,

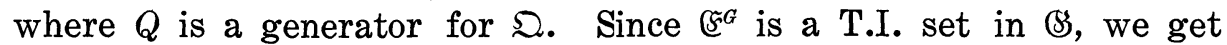
$\mathfrak{Q} \subseteq N\left(\mathfrak{F}^{G}\right)$, and so $\mathfrak{C} \subseteq\left(\mathfrak{F}^{G}\right)$. This then forces $\mathfrak{Q}$ to centralize [₹్,U], against $|[\mathfrak{F}, \Re] \cap C(\mathfrak{Q})|=2^{2},|[\mathfrak{F}, U]|=2^{3}$, and [₹, $\left.U\right] \subset[\mathfrak{F}, \Re]$.

We conclude that $\sigma_{2}=\varnothing$.

Received May 1, 1972. 



\section{PACIFIC JOURNAL OF MATHEMATICS}

\section{EDITORS}

RICHARD ARENS (Managing Editor) University of California

Los Angeles, California 90024

R. A. BeaUmont

University of Washington Seattle, Washington 98105
J. DUGUNDJI*

Department of Mathematics University of Southern California Los Angeles, California 90007

D. Gilbarg and J. Milgram Stanford University

Stanford, California 94305

\section{ASSOCIATE EDITORS}

E. F. BECKENBACH

B. H. NeUmanN

F. WOLF

K. YoSHIDA

\section{SUPPORTING INSTITUTIONS}

\section{UNIVERSITY OF BRITISH COLUMBIA CALIFORNIA INSTITUTE OF TECHNOLOGY UNIVERSITY OF CA.LIFORNIA MONTANA STATE UNIVERSITY UNIVERSITY OF NEVADA NEW MEXICO STATE UNIVERSITY OREGON STATE UNIVERSITY UNIVERSITY OF OREGON OSAKA UNIVERSITY}

UNIVERSITY OF SOUTHERN CALIFORNIA STANFORD UNIVERSITY UNIVERSITY OF TOKYO UNIVERSITY OF UTAH WASHINGTON STATE UNIVERSITY UNIVERSITY OF WASHINGTON AMERICAN MATHEMATICAL SOCIETY NAVAL WEAPONS CENTER

* C. R. DePrima California Institute of Technology, Pasadena, CA 91109, will replace J. Dugundji until August 1974. 


\section{Pacific Journal of Mathematics}

\section{Vol. 50, No. $1 \quad$ September, 1974}

Gail Atneosen, Sierpinski curves in finite 2-complexes.............. 1

Bruce Alan Barnes, Representations of $B^{*}$-algebras on Banach spaces .... 7

George Benke, On the hypergroup structure of central $\Lambda(p)$ sets ....... 19

Carlos R. Borges, Absolute extensor spaces: a correction and an

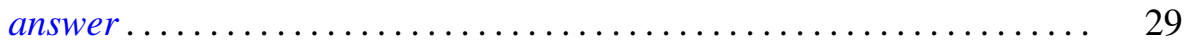

Tim G. Brook, Local limits and tripleability .................. 31

Philip Throop Church and James Timourian, Real analytic open maps .... 37

Timothy V. Fossum, The center of a simple algebra ............... 43

Richard Freiman, Homeomorphisms of long circles without periodic

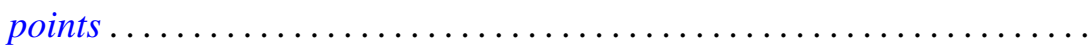

B. E. Fullbright, Intersectional properties of certain families of compact

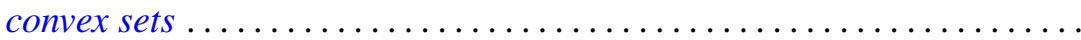

Harvey Charles Greenwald, Lipschitz spaces on the surface of the unit

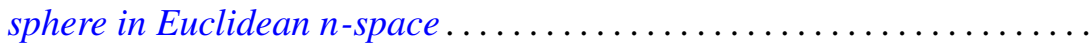

Herbert Paul Halpern, Open projections and Borel structures for

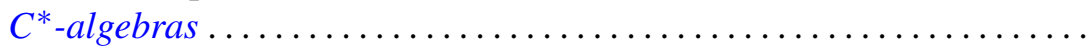

Frederic Timothy Howard, The numer of multinomial coefficients divisible

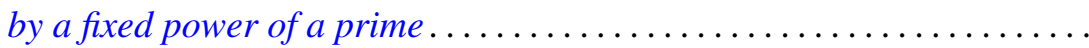

Lawrence Stanislaus Husch, Jr. and Ping-Fun Lam, Homeomorphisms of manifolds with zero-dimensional sets of nonwandering points........ 109

Joseph Edmund Kist, Two characterizations of commutative Baer rings ...

Lynn McLinden, An extension of Fenchel's duality theorem to saddle functions and dual minimax problems ................

Leo Sario and Cecilia Wang, Counterexamples in the biharmonic classification of Riemannian 2-manifolds...

Saharon Shelah, The Hanf number of omitting complete types ...

Richard Staum, The algebra of bounded continuous functions into a

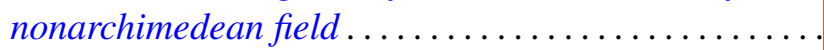

James DeWitt Stein, Some aspects of automatic continuity ..

Tommy Kay Teague, On the Engel margin

John Griggs Thompson, Nonsolvable finite groups all of whose local subgroups are solvable, $V \ldots \ldots \ldots \ldots \ldots \ldots \ldots \ldots$

Kung-Wei Yang, Isomorphisms of group extensions 